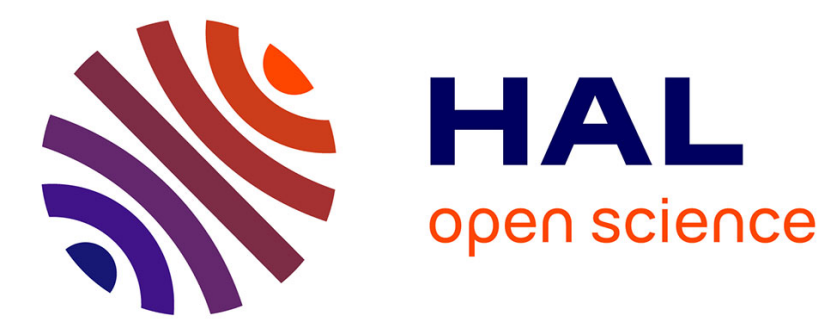

\title{
Spatial aggregation issues in traffic assignment models
}

Ouassim Manout

\section{To cite this version:}

Ouassim Manout. Spatial aggregation issues in traffic assignment models. Economics and Finance. Université de Lyon, 2019. English. NNT : 2019LYSE2014 • tel-02278052

\section{HAL Id: tel-02278052 \\ https://theses.hal.science/tel-02278052}

Submitted on 4 Sep 2019

HAL is a multi-disciplinary open access archive for the deposit and dissemination of scientific research documents, whether they are published or not. The documents may come from teaching and research institutions in France or abroad, or from public or private research centers.
L'archive ouverte pluridisciplinaire HAL, est destinée au dépôt et à la diffusion de documents scientifiques de niveau recherche, publiés ou non, émanant des établissements d'enseignement et de recherche français ou étrangers, des laboratoires publics ou privés. 


\section{UNIVERSITÉ -université \\ (U) DE LYON}

No d'ordre NNT : 2019LYSE2014

\section{THESE de DOCTORAT DE L'UNIVERSITÉ DE LYON}

Opérée au sein de

L'UNIVERSITÉ LUMIÈRE LYON 2

École Doctorale : ED 486 Sciences Économique et de Gestion

Discipline : Sciences économiques

Soutenue publiquement le 8 avril 209, par :

\section{Ouassim Manout}

\section{Spatial aggregation issues in traffic assignment models.}

Devant le jury composé de :

Aura REGGIANI, Professeure, Université de Bologne, Présidente

Julie LE GALLO, Professeure, AgroSup Dijon, Rapporteure

Thierry BLAYAC, Professeur des universités, Université de Montpellier, Rapporteur

Louafi BOUZOUINA, Chargé de recherche, École Nationale des Travaux Publics de l'État, Examinateur

Frédéric DERKX, Directeur de modélisation, ForCity (Financeur CIFRE), Examinateur

Patrick BONNEL, Ingénieur de Recherche, École Nationale des Travaux Publics de l'État, Directeur de thèse 


\section{Contrat de diffusion}

Ce document est diffusé sous le contrat Creative Commons « Paternité - pas de

modification »: vous êtes libre de le reproduire, de le distribuer et de le communiquer au public à condition d'en mentionner le nom de l'auteur et de ne pas le modifier, le transformer ni l'adapter. 


\title{
UNIVERSITÉ (U) DE LYON \\ UNIVERSITÉ DE LYON \\ UNIVERSITÉ LUMIÈRE LYON 2
}

ÉCOLE DOCTORALE DES SCIENCES ÉCONOMIQUES ET DE GESTION (EDSEG)

\section{SPATIAL AGGREGATION ISSUES IN TRAFFIC ASSIGNMENT MODELS}

\author{
A THESIS PRESENTED BY \\ OUASSIM MANOUT \\ IN FULFILLMENT OF THE REQUIREMENTS \\ FOR THE DEGREE OF DOCTOR IN SCIENCES
}

Supervised by:

Prof. Patrick Bonnel

Ph.D Louafi Bouzouina

Thesis committee:

Prof. Thierry Blayac, University of Montpellier, CEE-M

Prof. Patrick Bonnel, University of Lyon, ENTPE, LAET

Ph.D. Louafi Bouzouina, University of Lyon, ENTPE, LAET

Mr. Frédéric Derkx, ForCity, Modeling Director

Prof. Julie Le Gallo, AgroSup Dijon, CESAER

Prof. Aura Reggiani, University of Bologna, Department of Economics

Lyon, France

April 2019 



\section{Acknowledgments}

This thesis owes its existence to Patrick Bonnel. From the first day when I had expressed my will to undergo a $\mathrm{PhD}$ thesis, Patrick has always been of great support and help to me and to this work. His invaluable scientific supervision, commitment, and kindness have made of this 40-month $\mathrm{PhD}$ a valuable experience, rich of scientific learning and discoveries.

I am also grateful to Louafi Bouzouina for being of advice to me. In his company, I have learnt a great deal on the academic macrocosm.

This $\mathrm{PhD}$ would not have seen the day without the goodwill and the support of ForCity. A special thanks to Frédéric Derkx, Hakim Ouaras, and François Pacull for their engagement with this thesis. Without the backing and the arrangements made by Frédéric, this $\mathrm{PhD}$ would not be as it is today. The help of François Pacull and the supervision of Hakim Ouaras have been gratefully appreciated.

I am also thankful to my research institutes: Laboratoire Aménagement Economie Transports (LAET), Ecole Nationale des Travaux Publics de l'Etat (ENTPE), and the University of Lyon for sparing no effort to offer the best conditions to achieve this work.

This PhD has been funded by ForCity, Agence Nationale de la Recherche et de la Technologie (ANRT), and Laboratoire Aménagement Economie Transports (LAET) under the CIFRE funding grant $N^{\circ} 2015 / 0341$. This financial support is gratefully acknowledged.

Finally, my feelings of gratitude go to my parents and to Catherine for their unfailing encouragements and patience. 


\section{Abbreviations}

ABM: Agent-Based Models

ANOVA: ANalysis Of VAriance

ARD: Absolute Relative Difference

ATD: Average Traveled Distance

BPR: Bureau of Public Roads

BRT: Bus Rapid Transit

BSU: Basic Spatial Units

CBA: Cost-Benefit-Analysis

CBD: Central Business District

DDF: Distance Decay Function

DUE: Deterministic User Equilibrium

FSM: Four Step Models

GEH: Geoffrey E. Havers indicator

GTFS: General Transit Feed Specification

INSEE: Institut National de la Statistique et des Etudes Economiques

IRIS: Ilots Regroupés pour l'Information Statistique

LAET: Laboratoire Aménagement Economie Transports

LUTI: Land-Use and Transport Interaction/Integration

LUTM: Land-Use and Transport Models

MAUP: Modifiable Areal Unit Problem

MCM: Modal Choice Model 
OLS: Ordinary Least Square

PRMSE: Percent Root Mean Square Error

RD: Relative Difference

RF: Reduction Factor

RIT: Rate of Intrazonal Trips

ROEPV: Ratio between Observed and Estimated Passenger Volumes

SUE: Stochastic User Equilibrium

TAZ: Traffic Analysis Zone

TR: Transfer Rate

UAL: Urban Area of Lyon

VDF: Volume-Delay Function

VKT: Vehicle-Kilometers of Travel 


\section{Summary}

Cities are complex systems that urban models can help to comprehend. From simplistic models to more sophisticated ones, urban models have pushed forward our understanding of urban phenomena and their intricacies. In this context, models can be of great value to policy-makers providing that these tools become practical. In this regard, research has put little emphasis on the practicality of urban models and their use under operational conditions. To date, urban models, which rely on spatial aggregation, are the closest possibility to come to practical models. That is partly why, the spatially aggregated modeling framework is widely used. This framework is relatively practical when compared to other modeling frameworks like microsimulation. Nevertheless, spatial aggregation in these models can be a serious source of modeling errors. This is especially the case of Land-Use and Transport Interaction (LUTI) models and more particularly of Four Step Models.

The current $\mathrm{PhD}$ is committed to the study of spatial aggregation issues in traffic assignment models and its impact on their practicality. Traffic assignment is used to compute travel times and travel conditions of present and future travel demand. Accessibility measurement, which is at the core of LUTI models, is tightly dependent on traffic assignment modeling and outcomes. Any bias in traffic assignment is likely to corrupt the overall modeling framework. In this context, a special attention is to be paid to spatial aggregation in traffic assignment models. In traffic assignment, spatial aggregation consists in grouping observations using traffic analysis zones instead of using a

continuous description of space. By design, aggregation bears an implicit omission in data variability and thus a potential bias if this omission is not random. This is the case with the definition of centroid connectors and the omission of intrazonal trips in traffic assignment.

With the use of zones as the basic spatial units, transport models require the use of centroid connectors to attach zones to the transportation network. Centroid connectors are introduced to model average access and egress conditions. To model these conditions, the majority of transport models relies on a crude method that is subject to spatial aggregation errors. The current $\mathrm{PhD}$ examines, in detail, the impact of spatial aggregation in transit access modeling and suggests a new modeling strategy to overcome some modeling errors induced by aggregation. The use of zones as spatial units induces, as well, a loss of intrazonal data. The omission of intrazonal trips in traffic assignment models is an example of such omission. This research introduces an uncertainty framework to investigate the statistical impact of ignoring intrazonal trips in traffic assignment 
models. Findings from this research are used to design a new assignment strategy that is more robust towards the omission bias and more generally towards the spatial aggregation bias. 


\section{Contents}

1 Introduction $\quad 13$

1.1 Cities, Policy, and Complexity . . . . . . . . . . . . . . . 13

1.2 Urban models as a support-aid tool . . . . . . . . . . . . . . . . 15

1.3 Motivations . . . . . . . . . . . . . . . . . . . 19

1.4 Research framework: the aggregation problem in LUTM . . . . . . . . . . . . . 21

1.4 .1 Standard LUTI models . . . . . . . . . . . . . . . . . . 21

1.4 .2 The aggregation problem . . . . . . . . . . . . . . . 22

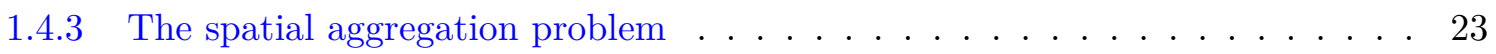

1.4 .4 Resolution strategies . . . . . . . . . . . . . . . . 26

1.4.5 Four Step Models and the spatial aggregation bias . . . . . . . . . . 28

1.5 Research questions and contribution . . . . . . . . . . . . . 31

1.5.1 Research questions . . . . . . . . . . . . . . . . 31

1.5.2 Thesis contribution . . . . . . . . . . . . . . . 32

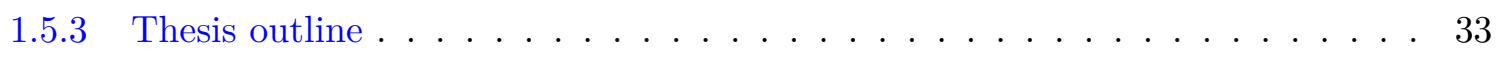

2 Sensitivity analysis on connectors $\quad 37$

2.1 Introduction . . . . . . . . . . . . . . . . . . 37

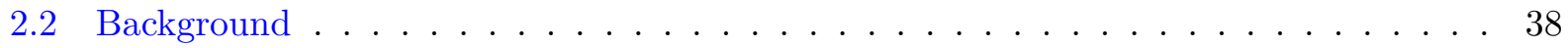

2.3 Methods . . . . . . . . . . . . . . . . . . . . 41

2.4 Data . . . . . . . . . . . . . . . . . . . . . . . . 42

2.4 Transit model of Lyon . . . . . . . . . . . . . . . . . . . . . . . . . . . . . . . . . . .

2.4 Experimental set-up . . . . . . . . . . . . . . . . . . . . . . . . . . . . . . .

2.5 Results . . . . . . . . . . . . . . . . . . . . . 45

2.5.1 Impact of connectors on the overall transit ridership . . . . . . . . . . . 45

2.5.2 The impact of connectors on modal ridership . . . . . . . . . . . . . . 47

2.6 Discussion . . . . . . . . . . . . . . . . . . 50

2.6.1 Correlation between transit ridership and $N_{\max } \ldots \ldots \ldots \ldots$. . . . . 50

2.6.2 Correlation between transit ridership and $L_{\max } \ldots \ldots \ldots \ldots \ldots$. . . . . . 51

2.6 .3 Impact on bus and rapid bus . . . . . . . . . . . . . . . . . . 51

2.6.4 Decreasing variability of results . . . . . . . . . . . . . 51 


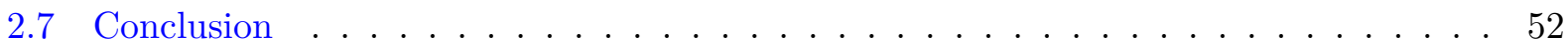

3 A new definition of transit connectors

3.1 Introduction . . . . . . . . . . . . . . . . . . . . 53

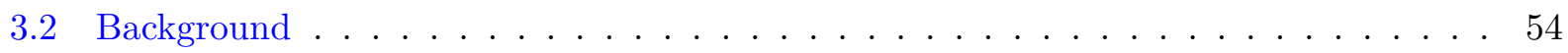

3.3 Methods . . . . . . . . . . . . . . . . . . . . 55

3.3 .1 The Algorithm . . . . . . . . . . . . . . . . . 56

3.3 .2 Justification . . . . . . . . . . . . . . . . . . . . . 58

3.4 A case study of Lyon . . . . . . . . . . . . . . . . . . . . . . 62

3.4.1 Validation protocol . . . . . . . . . . . . . . . 65

3.4.2 Results and discussion . . . . . . . . . . . . . . . . 66

3.5 Conclusion: policy implications . . . . . . . . . . . . . . . . 71

4 The omission of intrazonal trips $\quad 75$

4.1 Introduction . . . . . . . . . . . . . . . . . . 75

4.2 Background . . . . . . . . . . . . . . . . . . 76

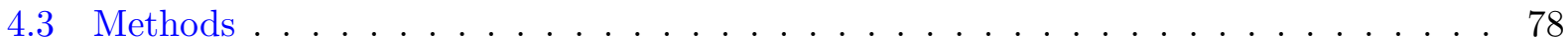

4.3 .1 Zonal Aggregation . . . . . . . . . . . . . . . . . 80

4.3 .2 Traffic Assignment . . . . . . . . . . . . . . . . . . . . . 82

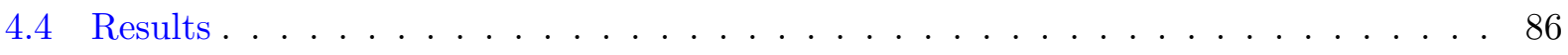

4.4 Impact on traffic . . . . . . . . . . . . . . . . . . 86

4.4 .2 Impact on journey speeds . . . . . . . . . . . . . . . . . . . 89

4.4.3 Impact on congestion ratio and total vehicle-kilometers of travel . . . . . . . 92

4.5 Discussion . . . . . . . . . . . . . . . . . . . . . . . . 92

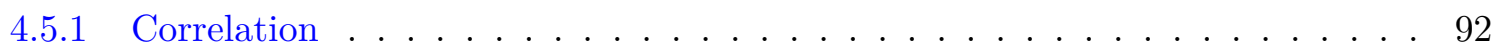

4.5.2 Asymptotic effect or threshold effect . . . . . . . . . . . . . . . . 95

4.5.3 Impact on the secondary network . . . . . . . . . . . . . . . . . 97

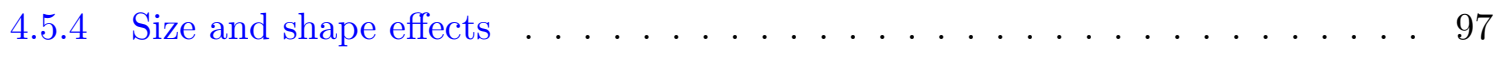

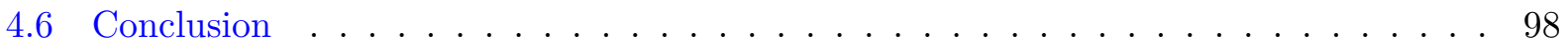

5 Spatial aggregation in assignment 101

5.1 Context . . . . . . . . . . . . . . . . . . . . . 101

5.2 Literature review . . . . . . . . . . . . . . . . . . . . . 103

5.2 .1 Demand-side methods . . . . . . . . . . . . . . . . . . 103

5.2 .2 Supply-side methods . . . . . . . . . . . . . . . . . . 105

5.2 .3 Mix methods . . . . . . . . . . . . . . . . . . . 106

5.3 Objectives . . . . . . . . . . . . . . . . . . . 107

5.4 Methods . . . . . . . . . . . . . . . . . 107

5.4 Traffic assignment strategies . . . . . . . . . . . . . . . 109

5.4 .2 Synthesis . . . . . . . . . . . . . . . . . . 115

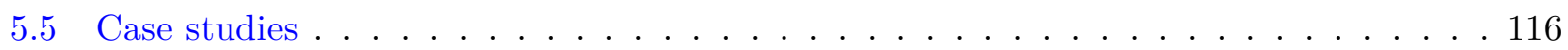


5.5.1 Simple case study: a 4 zones 8 links example . . . . . . . . . . . 116

5.5 .2 Sioux Falls case study . . . . . . . . . . . . . . . 116

5.5 .3 Lyon case study . . . . . . . . . . . . . . . . . . . . . . 118

5.6 Results . . . . . . . . . . . . . . . . . . . . . . 121

5.6.1 First case study: the 4 zones 8 links example . . . . . . . . . . . . 123

5.6 .2 Sioux Falls case study . . . . . . . . . . . . . . . . . . . 133

5.6 .3 Lyon case study . . . . . . . . . . . . . . . . . . 143

5.7 Discussion . . . . . . . . . . . . . . . . . . . . . . . 155

5.7 .1 Contribution of new assignment strategies . . . . . . . . . . . 156

5.7 .2 The rerouting problem . . . . . . . . . . . . 156

5.7 .3 Why a U-shape? . . . . . . . . . . . . . . . . . . . . 159

5.7.4 The artifact behind the outperformance of method 4.1 over $4.2 \ldots \ldots 1$

5.8 Conclusion and perspectives . . . . . . . . . . . . . . . . . 162

6 General discussion $\quad 167$

6.1 Thesis overview . . . . . . . . . . . . . . . . . . . 167

6.2 Contribution and recommendations . . . . . . . . . . . . . . 169

6.2.1 A new definition of transit connectors . . . . . . . . . . . . . . 169

6.2.2 The impact of ignoring intrazonal trips in traffic assignment models . . . . . 171

6.2.3 The spatial aggregation problem in traffic assignment models . . . . . . . . 174

6.2 .4 General contribution . . . . . . . . . . . . . . . . 176

6.3 Shortcomings and future work . . . . . . . . . . . . . 176

6.3 .1 Incomplete work . . . . . . . . . . . . . . . . 176

6.3.2 Spatial aggregation, detailed data, and microsimulation . . . . . . . . 177

6.3 .3 Global contribution . . . . . . . . . . . . . . . . 178

6.3.4 Consistency between space and network . . . . . . . . . . . . . . . 179

$\begin{array}{ll}\text { References } & 181\end{array}$

7 Appendix $\quad 197$

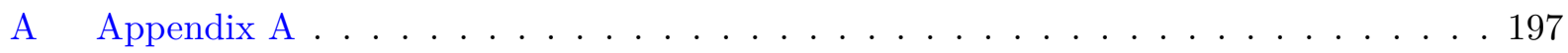

A.1 Zoning 2: zones $1,3,4,11,12,13,14,23,24 \ldots \ldots \ldots$

A.2 Zoning 1: zones 1, 3, 4, 11, $12 \ldots \ldots \ldots \ldots \ldots \ldots \ldots \ldots \ldots \ldots \ldots \ldots$

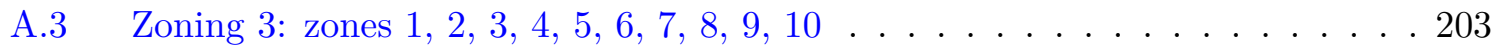

A.4 Zoning 4: zones 13, 14, 15, 19, 20, 21, 22, 23, $24 \ldots \ldots$. . . . . . 204

A.5 Zoning 5: zones 2, 5, 6, 7, 8, 9, 10, 16, 17, $18 \ldots \ldots \ldots$

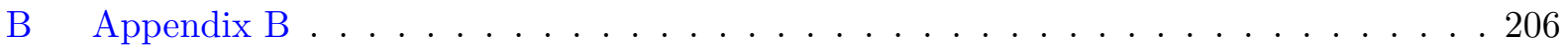

8 Contents 209 


\section{List of Figures}

1.1 Conventional Four Step Model architecture . . . . . . . . . . . . . . . 16

1.2 Land-Use and Transport interaction models according to Wegener (1998) . . . . . 17

1.3 Research summary . . . . . . . . . . . . . . . . . . . . 35

2.1 The standard definition of centroid connectors. Only the $N$ nearest stopping points within a buffer of radius $L$ around zone centroid are connected. . . . . . . . . . . . 38

2.2 The Metropolitan Area of Lyon and its transit system . . . . . . . . . . . . . . 43

2.3 Impact of transit connectors on total transit ridership. X-axis depicts the maximum number of connectors $N_{\max }$. The Y-axis depicts corresponding transit ridership. Three values are used for $L_{\max }: 500,1,000$, and 1,500 meters $\ldots$. . . . . . . . . . 46

2.4 The p-value of statistical tests of $H_{0}$ and $H_{0}^{\prime}$ on mean and variance equality. X-axis depicts the maximum number of connectors $N_{\max } \ldots \ldots \ldots$. . . . . . . 47

2.5 Statistical distribution of the impact of transit connectors on total ridership of (a) bus and (b) rapid bus systems. X-axis depicts the maximum number of connectors $N_{\max }$. The Y-axis depicts corresponding transit ridership. Three values are used for $L_{\max }: 500,1,000$, and 1,500 meters $\ldots \ldots \ldots \ldots \ldots$. . . . . . . . 48

2.6 The statistical distribution of the impact of transit connectors on total ridership of (a) light rail and (b) subway systems. X-axis depicts the maximum number of connectors $N_{\max }$. The Y-axis depicts corresponding transit ridership. Three values are used for $L_{\max }: 500,1,000$, and 1,500 meters . . . . . . . . . . . . . . . . . . 49

2.7 Impact of transit connectors on transfers of all transit modes. X-axis depicts the maximum number of connectors $N_{\max }$. The Y-axis depicts corresponding transit transfers. Three values are used for $L_{\max }: 500,1,000$, and 1,500 meters . . . . . . 50

3.1 The new definition of transit connectors: the algorithm $\ldots \ldots \ldots$

3.2 Selection of connection nodes. Transit routes A and B serve 4 transit stops and 4 zones. Route A serves stops S1-A, S2-A and S3-A. Route B serves stops S3-B and S4-B 59

3.3 The Urban Area of Lyon and its transit system (limits of 1999) (Sources IGN, Métropole de Lyon) . . . . . . . . . . . . . . . . . . . 63

3.4 Distance decay functions by transit mode (Source: RATP data) . . . . . . . . . . . 64 
3.5 Observed and estimated total transit journeys by mode. The bars are displayed in the order of the legend . . . . . . . . . . . . . . . 67

3.6 PRMSE between predicted and observed transit ridership by mode. The bars are displayed in the order of the legend . . . . . . . . . . . . . 6 68

3.7 The ratio between observed and estimated passenger volumes (ROEPV) $\ldots \ldots$. . . 69

4.1 The experimental protocol. The study area is divided using different spatial designs. For each zoning an assignment procedure is computed and the variability of results is analyzed . . . . . . . . . . . . . . . . . . . . 80

4.2 The urban area of Lyon (limits of 1999). The urban area is divided into 777 TAZ (IRIS) (data produced by Laboratoire Aménagement Économie Transports and IGN: Institut national de l'information géographique et forestière) . . . . . . . . . . . 82

4.3 The transport network of the Urban Area of Lyon . . . . . . . . . . . . . . . . 83

4.4 Conservation of connectors during zonal aggregation. Four zones: A, B, C, and D connected to their corresponding connection nodes: a, b, c, and d with connectors A-a, B-b, C-c, and D-d respectively. The connectors of macro-zone E are the set of the connectors of micro-zones $\mathrm{C}$ and D (Source: adapted from a suggestion of a reviewer of the paper $\ldots \ldots \ldots \ldots \ldots \ldots$

4.5 Correlation between average link flows and intrazonal trips according to road category. $\mathrm{X}$-axis represents the rate of un-assigned intrazonal trips and $\mathrm{Y}$-axis depicts average link flows on the network. The mean is computed using the length of links as a weight. Each point in the graphic is a traffic assignment outcome . . . . . . . . . .

4.6 Impact of the level of zonal aggregation on average links flows according to road category. $\mathrm{X}$-axis represents zonal aggregation level and Y-axis depicts average link flows. The mean is computed using the length of links as a weight. For each level of aggregation, 50 alternatives are tested $\ldots \ldots \ldots \ldots$

4.7 Correlation between average link speeds and intrazonal trips according to road category. $\mathrm{X}$-axis represents the rate of the un-assigned intrazonal trips and $\mathrm{Y}$-axis depicts average link speed. The mean is computed using the length of links as a weight. Each point in the graphic is a traffic assignment outcome . . . . . . . . . .

4.8 Impact of level of zonal aggregation on average link speeds according to road category. $\mathrm{X}$-axis represents zonal aggregation level and $\mathrm{Y}$-axis depicts average link speed. The mean is computed using the length of links as a weight. For each level of aggregation, 50 alternatives are tested . . . . . . . . . . . . . . . . . . 91

4.9 Correlation between congestion ratio and intrazonal trips according to road category. $\mathrm{X}$ axis represents the rate of the un-assigned intrazonal trips and $\mathrm{Y}$ axis depicts congestion ratio. The mean is computed using the length of links as a weight. Each point in the graphic is a traffic assignment outcome . . . . . . . . . . . . . . . 
4.10 Correlation between total vehicle kilometers of travel (VKT) and intrazonal trips according to road category. $\mathrm{X}$ axis represents the rate of the un-assigned intrazonal trips and $\mathrm{Y}$ axis depicts total VKT. Each point in the graphic is a traffic assignment

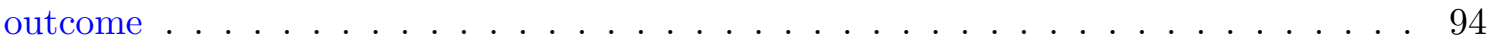

4.11 Intrazonal trips and zonal aggregation levels. For each level of aggregation, 50 alternative spatial designs are constructed . . . . . . . . . . . . . . 96

5.1 First case study: 4 zones, 8 links . . . . . . . . . . . . . . . . . 108

5.2 A model of traffic assignment models . . . . . . . . . . . . . . . . . . 109

5.3 Sioux Falls network Wang et al. (2013) . . . . . . . . . . . . . . . . 117

5.4 Original zoning of the case study of Lyon . . . . . . . . . . . . . . . 119

5.5 The transportation network of Lyon . . . . . . . . . . . . . 120

5.6 Distribution of the rate of intrazonal trips according to the aggregation level . . . 121

5.7 Simple case: PRMSE of link flows of the 6 assignment strategies. Color bars are displayed in the order of the legend. The PRMSE of Method 4.2 is nearly $0 \ldots 131$

5.8 Sioux Falls: original link flows. Link flows may differ from those known in the literature due to differences in convergence criteria . . . . . . . . . . . . 134

5.9 Sioux Falls: the relative difference (RD) of link flows produced by method 1. . . . 135

5.10 Sioux Falls: the relative difference (RD) of link flows produced by method 2.1 . . . 137

5.11 Sioux Falls: the relative difference (RD) of link flows produced by method 4.1 . . . 139

5.12 Sioux Falls: the relative difference (RD) of link flows produced by method 4.2 . . . 140

5.13 Sioux Falls: the PRMSE of link flows of the six assignment methods. Color bars are displayed in the order of the legend . . . . . . . . . . . . . . . . . 141

5.14 Lyon: correlation between link flows and intrazonal trips of method 1 according to road hierarchy. The $\mathrm{X}$-axis represents the rate of intrazonal trips and $\mathrm{Y}$-axis depicts average link flows. The mean is computed using the length of links as a weight . . . 146

5.15 Lyon: correlation between Vehicle-Kilometers of Travel (VKT) and intrazonal trips on the overall network of assignment method 1 . The $\mathrm{X}$-axis represents the rate of intrazonal trips and Y-axis depicts total vehicle-kilometers of travel on the network . 147

5.16 Lyon: correlation between link flows and intrazonal trips of methods 1 and 4.1 according to road hierarchy. The $\mathrm{X}$-axis represents the rate of intrazonal trips and Y-axis depicts average link flows. The mean is computed using the length of links as a weight . . . . . . . . . . . . . . . . . . . . . 148

5.17 Lyon: Correlation between link flows and intrazonal trips of methods 1, 4.1, and 4.2 according to road hierarchy. The $\mathrm{X}$-axis represents the rate of intrazonal trips and Y-axis depicts average link flows. The mean is computed using the length of links as a weight . . . . . . . . . . . . . . . . . . . . 150

5.18 Lyon: correlation between Vehicle-Kilometers of Travel (VKT) and intrazonal trips of methods 1, 4.1, and 4.2. The $\mathrm{X}$-axis represents the rate of intrazonal trips and Y-axis depicts vehicle-kilometers of travel on the network . . . . . . . . . . . 151 
5.19 Lyon: PRMSE of link flows of methods 1, 4.1, and 4.2 on road categories 1, 2, and 3. Each point is a PRMSE value of an assignment simulation . . . . . . . . . 153

5.20 Lyon: PRMSE of link flows of methods 1, 4.1, and 4.2 on road categories 4 and 5. Each point is a PRMSE value of an assignment simulation . . . . . . . . . . . . 154

5.21 Lyon: significance levels of rejection of the Null Hypothesis of equal means of Welch's

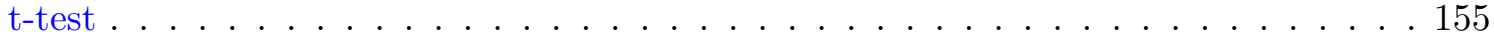

5.22 A sketch example of the rerouting problem . . . . . . . . . . . . 157

5.23 Lyon: Average Traveled Distance in Km function of intrazonal trips. The X-axis represents the rate of intrazonal trips and $\mathrm{Y}$-axis depicts the average distance traveled by cars in kilometers . . . . . . . . . . . . . . . . . . . . 158

5.24 lyon :Total Vehicle-Kilometers of Travel on the network . . . . . . . . . . . 159

5.25 The U-shape of assignment outcomes . . . . . . . . . . . . . . . . 160

7.1 Zoning 2: the relative difference (RD) of link flows produced by method 2.2 on the Sioux Falls case study ． . . . . . . . . . . . . . . . . . . . 198

7.2 Zoning 2: the GEH indicator of link flows produced by method 2.2 on the Sioux Falls case study . . . . . . . . . . . . . . . . . . . 199

7.3 Zoning 2: the relative difference $(\mathrm{RD})$ of link flows produced by method 3 on the Sioux Falls case study . . . . . . . . . . . . . . . . . . . . 200

7.4 Zoning 2: the GEH indicator of link flows produced by method 3 on the Sioux Falls case study . . . . . . . . . . . . . . . . . . . . . . . . 201

7.5 Zoning 1: PRMSE of assignment results on the Sioux Falls case study . . . . . . . 202

7.6 Zoning 3: PRMSE of assignment results on the Sioux Falls case study . . . . . . . 203

7.7 Zoning 4: PRMSE of assignment results on the Sioux Falls case study . . . . . . . . 204

7.8 Zoning 5: PRMSE of assignment results on the Sioux Falls case study . . . . . . . . 205

7.9 Median GEH indicator of the three assignment methods on road categories 1, 2, and 3207

7.10 Median GEH indicator of the three assignment methods on road categories 4 and 5 . 208 


\section{List of Tables}

2.1 Network characteristics of the Lyon transit system (GTFS, Métropole de Lyon version

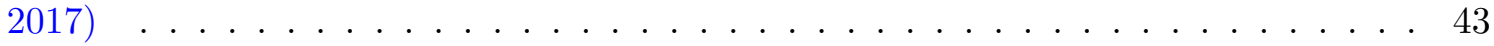

2.2 Travel time factors. . . . . . . . . . . . . . . . . . . 44

3.1 Accessible connection nodes and routes of the example in figure $3.2 \ldots \ldots$

3.2 Experimental design . . . . . . . . . . . . . . . . . . 65

3.3 Transfer rate of the different configurations . . . . . . . . . . . . . . 69

4.1 Summary of the current state of knowledge on the impact of intrazonal trips on transport modeling results. Only, relevant papers published before January 2018 are considered. Number between brackets are . . . . . . . . . . . . . . . 79

4.2 Characteristics of the network of Lyon according to road hierarchy. Mean values are computed using the arithmetic mean f . . . . . . . . . . . . . 83

5.1 Demand matrix of the simple case study f . . . . . . . . . . . 108

5.2 Uniform capacity of centroid connectors of the simple case study according to method

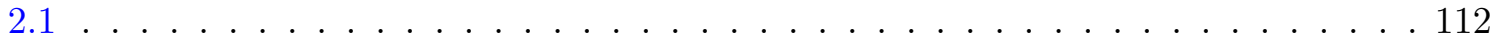

5.3 Original capacity of centroid connectors of the simple case study according to method

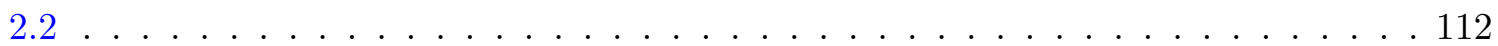

5.4 Trip demand matrix according to method $4.1 \ldots \ldots \ldots$. . . . . . . . . 114

5.5 Trip demand matrix according to method $4.2 \ldots \ldots \ldots$

5.6 Synthetic definition of the four assignment methods . . . . . . . . . . 116

5.7 Aggregated spatial designs of the Sioux Falls case study . . . . . . . . . . . 117

5.8 The hierarchy of the transportation network in the case of Lyon. Average capacity and speed are weighted by the length of links . . . . . . . . . . . . . 120

5.9 GEH recommendations (Great Britain and Highways Agency, 1996; Smith and

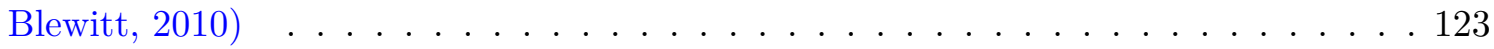

5.10 Simple case study: original assignment results _ . . . . . . . . . . . . 123

5.11 Simple case: assignment results of method 1. Reference flows of connector links 5-1 and 5-2 are Not Available (NA) 
5.12 Simple case: assignment results of method 2.1. Reference flows of connector links 5-1 and $5-2$ are Not Available $(\mathrm{NA}) \ldots \ldots \ldots \ldots \ldots$

5.13 Simple case: assignment results of method 2.2. Reference flows of connector links 5-1 and $5-2$ are Not Available $(\mathrm{NA}) \quad \ldots \ldots \ldots \ldots \ldots \ldots$

5.14 Simple case: assignment results of method 3. Reference flows of connector links 5-1 and $5-2$ are Not Available $(\mathrm{NA}) \quad \ldots \ldots \ldots \ldots \ldots \ldots$

5.15 Simple case: assignment results of method 4.1. Reference flows of connector links 5-1 and $5-2$ are Not Available $(\mathrm{NA}) \quad \ldots \ldots \ldots \ldots$. . . . . . . . . . . . . . . . . . . . . . . .

5.16 Simple case: assignment results of method 4.2. Reference flows of connector links 5-1 and $5-2$ are Not Available $(\mathrm{NA}) \ldots \ldots \ldots \ldots \ldots$

5.17 Original travel demand of the rerouting example . . . . . . . . . . . . 132

5.18 Assignment results of the rerouting example . . . . . . . . . . . . 132

5.19 Sioux Falls: descriptive statistics of the distribution of the Absolute Relative Difference (ARD) on network links . . . . . . . . . . . . . . . . . . 142

5.20 Sioux Falls: descriptive statistics of the distribution of the GEH on network links . 142

5.21 Assignment methods of the Lyon case study . . . . . . . . . . . . . . . . 143

5.22 Descriptive statistics of reference link flows according to road category. Average link flows and deviation are computed using the length of links as a weighting factor . . 144

5.23 Lyon case study: additional tests to explore the sensitivity of assignment results towards intrazonal trips distribution . . . . . . . . . . . . . . . . 162

5.24 Assignment results of tests 4.3 and 4.4 compared to original results of methods 4.1

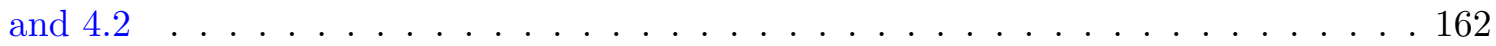




\section{Chapter 1}

\section{Introduction}

\subsection{Cities, Policy, and Complexity}

In 1950 , the rate of urban population was of $30 \%$; by 2050 , nearly $70 \%$ of world population will live in urban places and will occupy less than $5 \%$ of the earth's land surface (United Nations and Social Affairs, 2018). Cities $^{1}$ have long attracted individuals and few regions concentrate daily humankind's activities. Why is it so? This question has been the subject of a plethora of academic debates coming from various scientific backgrounds including economics. In urban economics and regional sciences, research is investigating the question of cities and the reasons behind their birth, growth, and even death (Alonso, 1964; Christaller, 1966; Fujita et al., 1999; Krugman, 1997; Marshall, 1895; Thünen, 1826; Venables, 2005; Weber, 1929). From an economic point of view, a city is a marketplace where different agents gather to produce, consume, and exchange goods and services. For cities to emerge and to become marketplaces, three conditions are needed (O'sullivan, 2007): agricultural surplus, urban production, and transportation infrastructures. Cities owe their existence to increasing productivity in agricultural production. From a historical point of view, agricultural surplus had freed labor force to occupy other productive activities like trade or manufacturing. In this context, urban dwellers are not self-sufficient and rely on agricultural surplus to fulfill their needs. In exchange, city dwellers offer different commodities to rural dwellers. Transportation infrastructures are required to allow such trade. However, for cities to thrive and to become metropolitan areas, the three previous prerequisites are not sufficient. Other conditions come into play, especially: scale economies and agglomeration economies.

Scale economies reward increasing scale in production operations. From an economic perspective, in the absence of scale economies in production, no city will ever exist and people will live in spatial

\footnotetext{
${ }^{1}$ The word city is used to depict an area with a high density of population relatively to surrounding places. In this chapter, words like: metropolitan areas, cities, or urban areas are used interchangeably. For a clear definition of these terms in the french context, refer to the administrative definition of l'INSEE.
} 
self-sufficiency with no need for trade, a situation known as backyard capitalism (Eaton and Lipsey cited in Fujita and Thisse (2003)). Due to input indivisibilities and labor specialization, scale economies induce decreasing marginal costs of production. Production plants are more efficient in producing goods than homemade production, everything else being equal. Therefore, small shops and manufacture places tend to increase their production scale to capture these economies. Scale economies emerge also in transactional costs inducing a reduction in transportation costs with increasing scales. One historic limitation to the size of cities derives from transportation costs. Shipping goods and commuting through space is costly and therefore has an impact on location choices of individuals and firms. Transportation costs often take part of main trade-offs facing individuals and firms in conducting their daily activities (commuting and delivery, for example) and long-run decisions (location, for example). With scale economies in transportation operations, transportation costs are reduced which in turn reinforces labor division, spatial specialization, and ultimately more concentration (Tabuchi and Thisse, 2011) ${ }^{2}$. Scale economies are therefore an essential ingredient in the development of cities. To this first ingredient, another force adds: agglomeration economies.

Agglomeration economies are defined as the pecuniary and non-pecuniary benefits that economic agents enjoy from spatially locating close to each other (Marshall, 1895). Agglomeration economies engender comparative advantages in clustered firms in comparison with isolated firms, everything else being equal. In this regard, these economies are essential to cities' formation and development. Agglomeration economies act in a self-reinforcing dynamic that is often divided in two types: localization economies and urbanization economies. 'Localization economies [...] are defined as the benefits generated by the proximity of firms producing similar goods; and [...] urbanization economies [...] are defined by all the advantages associated with the overall level of activity prevailing in a particular area' (Fujita et al., 1999; Hoover, 1937). Both types of economies enhance productivity and push toward agglomeration. Interaction between firms, availability of qualified workforce, reduced prices of inputs and transportation costs are examples of these benefits. Agglomeration economies benefit also to urban dwellers and final consumers by offering easy access to products, the ability to compare goods, and competitive job offers. For all these reasons and others, cities attract people and firms.

By attracting more individuals and jobs, cities are likely to experience increasing congestion, pollution, crime, spatial and social inequities along with their dramatic consequences for social welfare. These effects are known as negative externalities and are an example of market failure. Externalities are by nature not accounted for in market transactions and are outside of its scope ${ }^{3}$. In this case, market equilibrium is not socially efficient and needs regulatory interventions to correct for its failures. When not addressed properly, these externalities can offset previous urban comparative

\footnotetext{
${ }^{2}$ From an economic point of view, the absence of transportation costs gives birth to a spatial equilibrium with only one city dubbed World megalopolis (Fujita et al., 1999). Firms and population tend to locate in a unique central place.

${ }^{3}$ See Pigou (2017) and Baumol and Oates (1988) for a discussion on externalities.
} 
advantages and threaten cities' development, attractiveness and ultimately existence. Efficient policy interventions to mitigate these effects are therefore imperative.

In this complex context, policy measures to ensure long-standing and thriving cities are not straightforward (Campbell, 1996; May et al., 2003). In their Decision-Makers' Guidebook, May et al. (2003) describe this situation as a combination of complex decision-making responsibilities, complex interactions, with multiples objectives and a wide range of options. In this context, decision-makers and urban planners are required to maintain the functioning of cities, to contribute to their attractiveness, to mitigate their negative externalities, and to fulfill population's needs and requests of well-being; all of this within a sustainable framework and under uncertainty and risks of failure. To meet this challenge, policy-makers should be first, informed about the measures to take, and second, about the direct and indirect consequences of the measures to be taken. For a long time, this informative role has been played by different technical experts and consultants from different expertise areas. However, with increasing complexity of cities, a more holistic and systemic understanding of the urban phenomena is needed. Otherwise, economic, social and political implications of any policy decision can be unpredictable, fail to meet its expected targets or, at worst, be counterproductive with counter-intuitive effects as demonstrated by literature (Flyvbjerg, 2007; Flyvbjerg et al., 2005; Skamris and Flyvbjerg, 1997). In this regard, policy-makers are seeking accurate decision-support tools to dispel some of the uncertainty at play and help them tailor efficient policy measures (Commissariat Général à la Stratégie et à la Prospective, 2013; Denant-Boèmont, 1994; Emberger et al., 2008; Hardy, 2011; May et al., 2003; Saujot et al., 2015). Urban models are an example of these tools.

\subsection{Urban models as a support-aid tool}

Currently, few tools are at the disposal of policy-makers and experts to tackle the aforementioned situation. This is especially the case in transportation and urban planning. In transportation planning, most transport projects are assessed using the traditional Four Step Models (FSM) (Bonnel, 2004; Ortúzar and Willumsen, 2011). These models were born in the 50s in the USA during the massive postwar investment period (see Chatzis (2013) and Dupuy (1975) for a historical review of FSM). In their early days, these models were car-oriented tools used exclusively to inform policy-makers about road investments to engage in to relieve car congestion. Afterward, other transportation modes like transit or active modes were included in these models. Nowadays, four step models are used to depict and predict transportation demand and flows of individuals and goods on different modal networks and at different spatial and time scales. To do so, FSM follow a sequential schema of four steps: Trip Generation, Distribution, Modal split, and Traffic Assignment (figure 1.1). Thanks to decades of academic research and field applications, these models have reached a remarkable scientific maturity and practicality. At the same time, several commercial software were introduced to the market making of these models a practical and affordable decision-support 
tool. Consequently, the four step paradigm is till today the most widespread modeling framework in transportation planning.

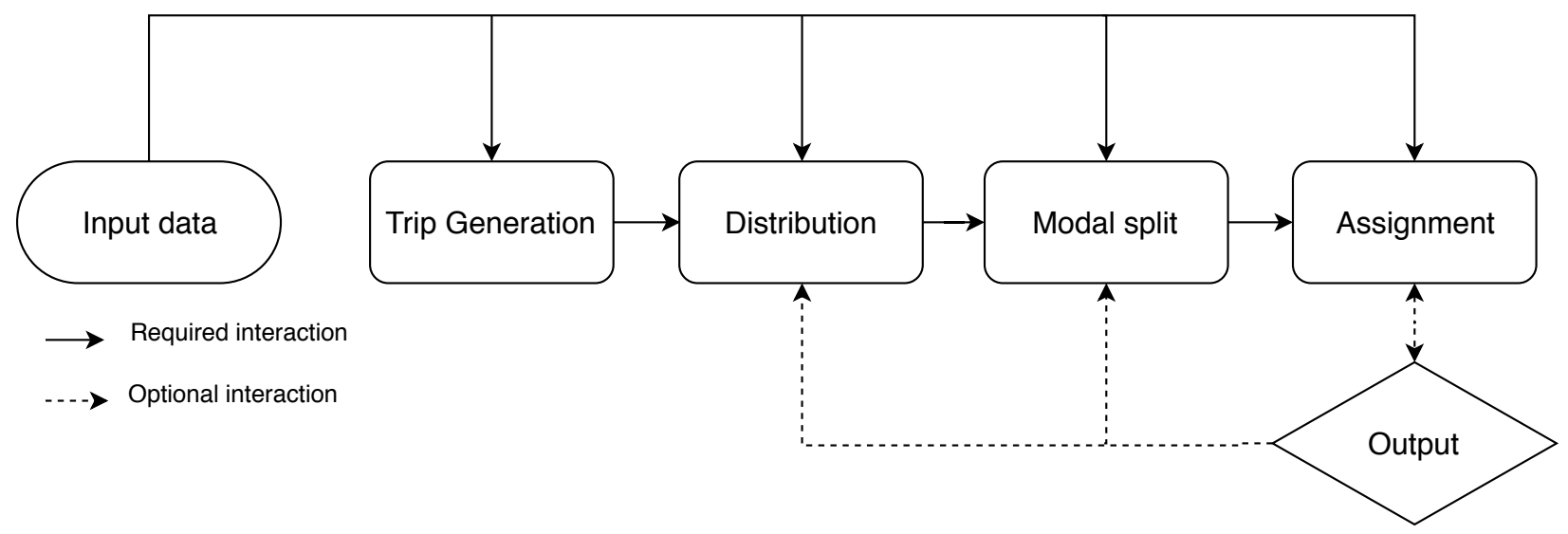

Figure 1.1: Conventional Four Step Model architecture

Despite its widespread adoption and use, research has early pointed out the flaws of this sequential modeling framework and the bias it may induce. One of these detrimental flaws is the lack of integration between the land-use system and the transportation system. As stressed before, space and networks are interrelated and need to be addressed as such. This is not the case with FSM. Four step models offer a truncated view of urban dynamics in the sense that the sequential architecture of FSM does not allow direct nor reciprocal interaction between the transportation and the land-use systems. Land-use data are only used as an exogenous input whose evolution is totally independent of the evolution of the transportation system. This is obviously a major shortcoming of the FSM approach that challenges the validity of this modeling framework especially in long-run and strategic planning where interactions between urban forms and mobility are decisive.

In parallel to the development of FSM, a new modeling approach has emerged: Land-Use and Transport Interaction or Integration (LUTI) models, known also as Land-Use and Transport Models (LUTM) (Simmonds et al., 1999; Southworth, 1995; Wegener, 2004, 1998; Wegener and Fuerst, 2004). In general terms, land-use stands for households' and firms' location and relocation processes together with urban development carried out by private and public initiatives. The transportation system describes network flows and their corresponding levels of service. LUTM recognize explicitly that land-use and transportation are co-determined dynamically with feedback between the two systems instead of the altered view embedded in FSM (figure 1.2). The interaction between these systems is central to the LUTI approach. In most LUTI models, the interaction between these two systems is embodied by the notion of accessibility, namely: the potential of opportunities for interaction (figure 1.2) (Hansen, 1959). These models build on different economic theories and on various research works (Geurs et al., 2012; Reggiani, 2019).

Practically, LUTM combine in the same modeling framework different modeling blocks that can be 
roughly divided into two modules: a land-use model and a transportation model ${ }^{4}$. This latter is often a four stage model. These modeling blocks are tied together using accessibility and feedback loops to ensure a dynamic interaction (year-by-year for example). Each module produces the input data required by the other module (figure 1.2). Moreover, the majority of LUTM pursue either an equilibrium or a disequilibrium approach: each simulation is an equilibrium search problem that can be reached in the case of equilibrium models or not as in the case of disequilibrium models. Due to this modular architecture, various research contributions have been made to develop accurate sub-modules: households' location choice models, firms' location choice models, relocation models, hedonic price models, etc.

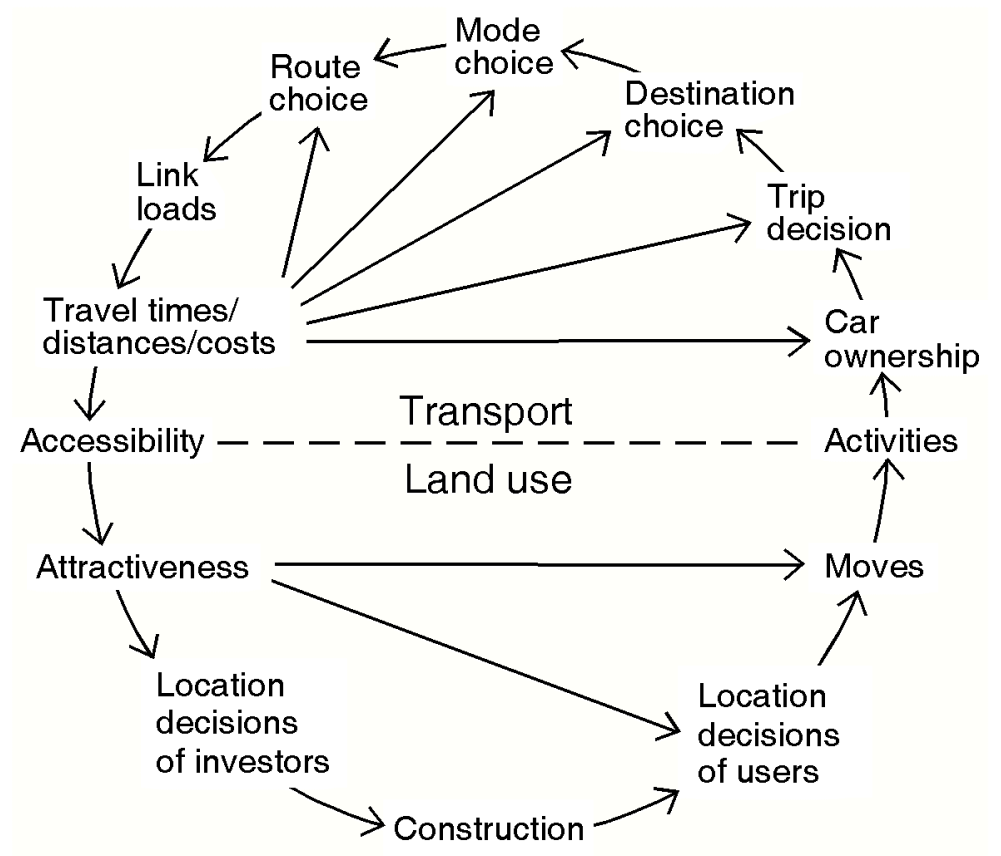

Figure 1.2: Land-Use and Transport interaction models according to Wegener (1998)

During the last decades, the LUTI approach has received great attention from a large research community and substantive advances have been made. According to Timmermans (2003), LUTM have followed three development stages. Early LUTI models from the 60 s to the 70 s were mainly aggregate spatial interaction models based on the economic base theory (North, 1955) or the input-output analysis (Leontief, 1986). The majority of these early-stage models were inspired by the seminal work of Lowry (1964). During that juvenile stage, high expectations were put in LUTM with sometimes unattainable promises delivered by modelers and urban planners. But like any juvenile modeling framework under-development, LUTM did not meet all their promises and have sometimes been the subject of criticism (Lee Jr, 1973). A decade later, a new generation of LUTI

\footnotetext{
${ }^{4}$ Some LUTI models like UrbanSim (Waddell, 2002) do not combine both modeling blocks but allow for such combination.
} 
models built on new findings in microeconomics has seen the day (Ben-Akiva and Lerman, 1985; McFadden, 1978). These models rely on the utility maximization approach applied to groups of agents and relying on an aggregate description of space (zones). In the sequel of this dissertation, these models are referred to as standard LUTI models as they are, till now, the most dominant LUTI approach. Due to increasing data availability and computational capacities, different standard modeling frameworks have been developed and tested in different urban contexts. Examples of these models are: UrbanSim (Waddell, 2002), DELTA (Simmonds, 1999), MEPLAN (Echenique et al., 1990), or TRANUS (de la Barra, 1999). These frameworks were applied in different case studies (see SIMBAD for an implementation of UrbanSim in Lyon (Nicolas et al., 2009)) and have delivered promising results for both, academics and practitioners. The third stage in the life course of LUTI models is called microsimulation. Microsimulation pushes forward the modeling resolution of standard LUTM to focus on individuals (i.e. agent-based models) and their activity patterns (activity-based models). The microsimulation framework cherishes the appealing idea that main city dynamics derive from individual decisions of agents in engaging in different activities. By modeling the key characteristics of these agents and their activities in space and time, microsimulation models attempt to better capture urban dynamics (see Timmermans (2003) for a review of microsimulation models). These models are promising and are still under development.

Thanks to years of development and research, LUTI models have reached outstanding scientific maturity that have pushed forward our understanding of urban organization. In this context, LUTI models have met, to a satisfactory degree, their first objective of understanding urban systems. However, despite the relative maturity some of these models have achieved, the LUTI framework is still unpractical, or at best, difficult to put into practice in real case studies that meet stakeholders' needs and constraints. This situation is detrimental to the widespread use of LUTM and an enduring source of debates and criticism about this modeling framework (Lee Jr, 1973; Saujot et al., 2015; Timmermans, 2003; Wegener, 2011). Consequently, few LUTM have made their way out of research institutes to conquer decision-making spheres and only few planning agencies around the world have used or are using LUTM in their daily missions ${ }^{5,6}$. In view of this situation, the LUTI approach fails to meet its second objective of improving urban planning by serving as a decision-support tool. In this regard, Waddell (2011) states: "Unfortunately, the task of implementing integrated land use, transportation and emissions planning within operational planning agency settings still faces formidable challenges".

${ }^{5}$ Models like UrbanSim (Waddell, 2002), TRANUS (de la Barra, 1999), MEPLAN (Echenique et al., 1990), or ILUT/DRAM/EMPAL (Putman, 1983 in Wegener (1998)) are an example of operational models that have been used in policy appraisal.

${ }^{6}$ One noteworthy example of a practical implementation of LUTM in France is the Grand Paris project where 3 LUTM: UrbanSim (Waddell, 2002), Pirandello (Delons et al., 2008) and Relu-Tran (Anas and Liu, 2007) have been used to assess the wider impacts of the Grand Paris project. 


\subsection{Motivations}

Despite the theoretical attractiveness of the LUTI approach, land-use and transport models are not yet fully adopted by decision-makers and the main barriers to this are (Saujot et al., 2015; Waddell, 2011; Wegener, 1998):

- The black-box effect or lack of transparency: integrated models together with the systemic approach may convey the idea of a black-box tool.

- Data requirements: a large amount of data is required by these models.

- Skill requirements: the development of a LUTM requires a wide range of sharp and specific skills.

- Calibration/Validation efforts: all LUTM need to be calibrated and validated before any operational use.

These barriers prevent LUTM from a wide adoption and use, especially those models of the second (standard models) and third generation (microsimulation models). At the same time, end-users and stakeholders are in need of such tools. This is a typical market mismatch situation where supply does not satisfy demand: for LUTM to be used as decision-support tools, these models should meet end-users needs and characteristics. In this regard, LUTM are still to convince policy-makers of their contribution given their cost. To do so, two strategies are, in our opinion, to be taken: (1) communication and popularization of LUTM; and (2) cost reduction of their implementation.

Regarding the first solution, in a review performed by Thomas et al. (2018), the authors found that only 21 empirical papers dealing with LUTI implementation in European cities ${ }^{7}$ have been published between 1990 and 2015. 5 out of 21 papers include a meta-analysis of different case studies for comparison purposes. The scarce literature on operational implementations of LUTM contrasts with the increasingly growing theoretical and empirical contributions published in numerous scientific journals. Whereas, both theoretical and empirical research streams are necessary and complementary in science, we notice, as other researchers do, that more applied research in LUTI models can foster their adoption by a public other than academics, namely: urban planners, and decision-makers, and ultimately enhance LUTI development and research agenda. To this end, a cost reduction of operational LUTM is vital. Nowadays, the implementation of these models is still costly and challenging. Surprisingly, limited if no research is exploring this path.

In regard of this situation, ForCity, a urban modeling start-up within which this $\mathrm{PhD}$ is undergone, has set out to conceive, to develop, and to implement a practical land-use and transport modeling framework for strategic and mid/long terms decision-support purposes. By practical, we mean:

- Useful: a tool to provide useful and reliable information given its scope and objectives.

\footnotetext{
${ }^{7}$ In this review, only European cities were considered. American and Asian cities were not included in this review (Thomas et al., 2018).
} 
- Replicable: a spatially transferable tool, easy to implement in different urban contexts and time periods.

- Affordable: within acceptable time-budget development and maintenance costs.

- Responsive: with reasonable computational times.

By useful, we mean a scientific sound modeling framework built on valid theoretical and behavioral assumptions. This framework is intended to provide useful and reliable answers to a predefined range of questions pertaining to the scope and objectives of the model. For this model to be used, it needs to be replicable in space and time. Replicability means here the ease with which a model can be applied to different urban contexts or different analysis periods. In this regard, model replicability is different from spatial or temporal transferability in the sense that, with replicability, the emphasis is more on the capacity and the ease of transferring the modeling architecture including data requirements rather than parameters' estimates. The affordability and responsiveness conditions are important in the development of practical models that are intended to be used in operational situations where end users have limited resources and reasonable expectations. These criteria are vital to the widespread use of any LUTM (Waddell, 2011; Wegener, 2011).

The state-of-the-art is scarce of examples of LUTM that respect all the criteria above, and this trend is not likely to change in a short or medium term. In fact, current research agendas are still investigating to push forward the accuracy and hence the complexity of these models with little regard to their implementation costs and practical use (see Miller (2018) and Wegener (2011) for a discussion on this subject). These efforts are, of course, vital to research. Pushing forward our understanding of cities and their dynamics is the primary objective of any urban research. Nonetheless, putting into practice these findings so that society can make advantage of it is also a worthwhile objective, especially in social sciences. In this regard, limited if no attention is put into the simplification of these models for the sake of end-users. As Wegener (2011) put it:

"[...] not all disaggregate urban and transport modelling projects have been successful (see, for instance, Wagner and Wegener, 200\%, Nguyen-Luong, 2008). Many large modelling projects failed to deliver in the time available or had to reduce their too ambitious targets. Many applications of established models by others than their authors did not become operational. Many projects got lost in data collection and calibration and did not reach the state of policy analysis. Many projects remained in the academic environment and produced only PhD theses."

"It seems however that a simplification of the [LUTI] approach will be counterintuitive. Any valid model should represent the key complexity of the phenomenon under investigation. The plea for behaviorally better models implies further complexity and many people will therefore continue to argue that the models are black boxes. There does not seem an easy solution to this dilemma."

Given this background, a joint research program between ForCity and the LAET research institute on urban planning and transport economics of the University of Lyon has been funded to explore the question of practical LUTM. The main aim of this program is to develop a strategic land-use 
and transport interaction modeling framework that complies, as far as possible, with practicality criteria and end-users' needs. From a scientific point of view, this project holds the promise of a significant contribution to urban research and especially to making of LUTM a well-established decision-support tool. Within this broad and ambitious research framework, the current $\mathrm{PhD}$ is undergone to contribute to the effort of making LUTM popular and practical tools. The current $\mathrm{PhD}$ research focuses on transportation models and particularly on assignment models.

In the next section, the $\mathrm{PhD}$ research questions are further developed.

\subsection{Research framework: the aggregation problem in LUTM}

\subsubsection{Standard LUTI models}

In order to develop strategic land-use and transport interaction models that satisfy practicality criteria and end-users' needs, the standard LUTI framework seems to be an appropriate research framework. This modeling framework relies on an aggregate description of agents, space, and time. By doing so, this framework seems to comply more conveniently with practicality criteria than other modeling frameworks. That is because, in the life course of LUTI modeling, standard LUTM have been under development and in use for more than 35 years. Consequently, these models have relatively reached satisfactory levels of maturity and practicality compared to other modeling frameworks like microsimulation. Furthermore, and more importantly, these models are convenient for the strategic modeling purposes of the current research where the emphasis is more on high level and global impacts rather than microscopic or local effects. In this context, standard LUTI models are also convenient for both explanatory and predictive purposes. In view of these reasons, we choose to investigate the issue of practicality within the standard LUTI modeling framework.

Standard LUTI models rely on an aggregate description of agents, time, or space, or all the three together. For these three dimensions, aggregation is often unavoidable since precise information about each agent in space and time is not always available; and if it comes to exist, this information is likely to be unreliable, especially in predictive situations. In this regard, aggregation is made for practicality reasons. Nevertheless, aggregation comes at a price of increased modeling errors. These errors are referred to as aggregation errors and can undermine the reliability of modeling results, their usefulness, and thereby their practicality. It is, therefore, necessary to investigate the aggregation problem in land-use and transport interaction models and the implications induced by such problem. The aggregation problem in LUTM is the research framework of this $\mathrm{PhD}$ dissertation. 


\subsubsection{The aggregation problem}

In economics, the aggregation problem arises whenever economists attempt to define a system of relations between an aggregate economic system (macroeconomics) and its underlying micro-level relations (microeconomics) (Aigner and Goldfeld, 1974; Cramer, 1964; Dunn et al., 1976; Malinvaud, 1956; Theil, 1954). In other words, aggregation relates to the problem arising from substituting a model (simple/aggregate) for another (detailed/complex model) (Malinvaud, 1956). This trade-off is not neutral and may induce bias. In this regard, we quote Hannan (1972): "[...] aggregation problems arise whenever an analyst makes inferences from a model estimated at one level of data aggregation to properties of an analogous model at a different level of aggregation". Aggregation comes at the price of a loss of information in the analysis whenever aggregate data are used to substitute individual or micro-level data (Clark and Avery, 1975). An aggregation or smoothing function is applied to agents, time, or space to infer new units of observation that are different from the original ones (Ijiri, 1971). The smoothing often induces a loss of information and a decrease in data variability. Consequently, results drawn from any aggregate model are potentially biased by this loss and will be different from those obtained at the original micro-level.

In LUTI models, the aggregation problem can arise from:

- Aggregation of behavior (agents).

- Aggregation of time (analysis periods).

- Aggregation of space (locations).

The aggregation of agents relates to the common modeling practice of grouping different agents like households or firms in groups of similar characteristics and supposedly of similar behavior ${ }^{8}$. This problem has been thoroughly studied in economics since the work of Theil (1954) (Aigner and Goldfeld, 1974; Cramer, 1964; Dunn et al., 1976; Malinvaud, 1993, 1956). Aggregation of time relates to the problem of conducting analysis over an indivisible period of time that may mask intra-period variability and dynamics (evening rush-hours in transportation modeling for example). This is a common practice in static models with contrast to dynamic models. The aggregation of space or locations, referred hereafter as spatial aggregation, describes the practice of modeling continuous space as discrete and of grouping agents' characteristics on the basis of discrete divisions. All spatial information is reduced to one point called zone centroid. Zones become the Basic Spatial Units (BSU) and all information lying inside these zones is often ignored or aggregated. For the three above dimensions: agents, time, and space, aggregation is undertaken for data availability or privacy issues $^{9}$. In other cases, aggregation can also be undertaken in line with modeling objectives: regional modeling for example. In all cases, the aggregation problem may potentially induce a bias

\footnotetext{
${ }^{8}$ The Aggregation Problem of agents has been studied in Geography as the Ecological Fallacy. This fallacy pertains to the practice of inferring individual relations from aggregate relations, which can be erroneous (see Robinson (1950)).

${ }^{9}$ Some models address the problem of data availability by using synthetic data.
} 
that can be detrimental to modeling accuracy and reliability. This bias may impede our efforts to build useful and reliable LUTI models within the standard modeling framework.

Aggregation problems in LUTI models are an interesting and rich research framework that one cannot cover entirely in a 40-month PhD. The current dissertation focuses exclusively on the Spatial Aggregation Problem. Aggregation of agents and time are of importance but the spatial aggregation problem bears on more closely to the research question at hand: how to enhance the practicality of LUTM? Spatial aggregation has direct implications on modeling usefulness, replicability, affordability, and responsiveness (see the section on Motivations). For a model to be useful, this latter should have an appropriate spatial resolution that is in line with its objectives and the questions under investigation. The spatial aggregation level of a model has also a straightforward influence on the amount of data required for the development and maintenance of this model. Coarse spatial models are more likely to require less detailed and more ubiquitous data than micro

level models. Also, calibration and validation efforts are deemed to be proportional to the spatial resolution of the model. Finally, and despite the advancements made in computation capacities, computational costs are still a limitation in complex modeling. In the case of spatial models, the computational burden stems partly from data storage and manipulation needs that are dependent on the spatial aggregation level. In this context, the spatial resolution of a model has a direct impact on its responsiveness.

All in all, the spatial aggregation problem seem to be a promising research framework to address the practicality issue in land-use and transport models. In the past, research dealing with spatial aggregation has often been undertaken from a computational efficiency perspective. Aggregation has retained the attention of science when the computational cost was a real burden. With technological advances and increasing data availability, science has lost its interest in aggregation. In this context, aggregation problems have been considered out of research scope since one can use detailed data and performant computers to get rid of such errors (Connors and Watling, 2014; Daganzo, 1980a; DeCorla-Souza and Grubb, 1991; Friesz, 1985). Nonetheless, the bias induced by aggregation may be enduring whatever micro-data or computers are used. It is then necessary to understand the impact of spatial aggregation errors and the possibility to neutralize them or at least to reduce their extent.

\subsubsection{The spatial aggregation problem}

By design, standard LUTI models do not capture all intrazonal variability and therefore are assumed to produce biased results when the omission is not accounted for (Atherton and Ben-Akiva, 1976; Ben-Akiva and Bolduc, 1987). This issue is commonly known as the Spatial Aggregation Problem and can be mathematically formulated.

Let $\varphi$ be a spatial phenomenon with a spatial distribution $f_{\varphi, S}$ observed in a spatial context $S$. $f_{\varphi, S}(p)$ is a measure of $\varphi$ in the spatial position $p$. In practice, precise information on $f_{\varphi, S}(p)$ is 
not always available nor reliable and one needs to use a less detailed modeling unit to capture $\varphi$. Spatial aggregation consists in defining:

- An aggregation function $G$ to perform aggregation on $f_{\varphi, S}$.

- A spatial division $D(S)$ of $S$ to perform aggregation over. $D(S)=\left[Z_{i}\right]_{1 \leq i \leq n}$ where $Z_{i}$ is a set of $n$ contiguous zones that divide $S$.

$\langle G, D(S)\rangle_{\varphi}$ is an aggregation system of $\varphi$ over $S$. This system is not unique: different spatial divisions can be defined for the study area $S$, as well as different aggregation functions. From this simple observation stem two noteworthy findings:

- In spatial modeling, outcomes depend on the aggregation system $\langle G, D(S)\rangle_{\varphi}$.

- In spatial modeling, one can get different modeling results when using different aggregation systems.

Given this aggregation system, the new population of observational units becomes:

$$
F_{\varphi}(G, D)=\left[G\left(f_{\varphi, Z_{i}}\right)\right]_{Z_{i} \in D(S)}
$$

It is clear that from equation $1.1, F_{\varphi}(G, D) \not \equiv f_{\varphi, S}$. The original continuous distribution $f_{\varphi, S}$ is different from the aggregate and artificial new observational units $F_{\varphi}(G, D)$. This mismatch is the main source of the spatial aggregation bias, and the extent of this problem depends on the extent of the mismatch. From equation 1.1, it is obvious that spatial aggregation errors are of two natures depending on:

1. The aggregation function $G$.

2. The spatial division $D$.

Assumptions on $G$ and $D$ have an impact on modeling outcomes. In economics, in general, and urban economics, in particular, these assumptions are often implicit and so does their impact on modeling outcomes.

\subsubsection{Aggregation function $G$}

Numerous research works apply the Mean or Median functions on data distribution to summarize information at the zonal level. This practice reduces data variability and induces an unavoidable loss of information (Ijiri, 1971). In transport modeling for instance, spatial location of trip makers is often summarized by an average position at the zone centroid. Errors induced by the aggregation function can be of different natures, two of them are of interest to our research: 
- Omission of information: aggregation functions operate like a frequency filter that discards totally or partly intrazonal information. Often, this omission is not random and it is a potential statistical bias. The omission of intrazonal trips in assignment models is an example of such omission.

- Aggregation of information: when the distribution of intrazonal data is not omitted, it is often transformed by an aggregation function. The aggregation of information comes at the price of a loss of information.

\subsubsection{Spatial division $D$}

To perform spatial aggregation, one needs a spatial division. To this end, different criteria can be used to construct a spatial delineation (see Martínez et al. (2009) for a comprehensive review on spatial division criteria of traffic analysis zones). Whatever criteria are used, three issues arise from spatial division:

1. Boundary effect: where a study area begins and where it ends?

2. Number of zones: is there an appropriate number of zones for a study area and a research question?

3. Shape of zones: for a predefined number of zones, different zoning designs can be defined. Is there a convenient shape for these zones?

The boundary effect relates to the problem of defining artificial and arbitrary boundaries for analysis purposes (Miller, 1999). This is a necessary assumption in spatial modeling. Practically, the study of any spatial phenomenon is often confined to a predefined study area. In this regard, the main issue underlying the boundary effect is that the study area is exactly delimited whereas the spatial phenomena at play are not. Spatial phenomena are fuzzy, subject to spatial autocorrelation and may interact beyond artificial boundaries (Le Gallo and others, 2000). Accordingly, the subject under study may be influenced by other factors outside the study area that the model cannot capture. To correct for this problem, different methods have been suggested (Anselin, 2013; Griffith, 1983; Stewart Fotheringham and Rogerson, 1993) (see Thomas et al. (2018) for a recent review of literature on city delineation in LUTI models). An appropriate boundary for the study of a spatial phenomenon should include all impactful factors pertaining to the phenomenon under investigation. In practice, only a part of these factors is known and to a lesser degree observable. To delineate a study area in LUTI models, Jones (2016) recommends using commuting flows (trip tables) and cluster analysis to group zones in order to maximize intra-area interactions and to minimize inter-area fluxes. This practice is found to produce delineations that embrace functional forms, to produce large study area extents, and to reduce the omitted influence of "the rest of the world".

The Modifiable Areal Unit Problem (MAUP) depicts the dependency between modeling results and the modifiable nature of spatial units, i.e. their shape and number. This means that different 
outcomes can be drawn from a model by only using different spatial designs. The MAUP has been extensively discussed in geography and long been ignored in economics (Amrhein, 1995; Batty and Sikdar, 1982a, 1982b, 1982c, 1982d; Briant et al., 2010; Clark and Avery, 1975; Fotheringham and Wong, 1991; Gehlke and Biehl, 1934; Openshaw, 1983, 1978, 1977a, 1977b; Wong, 2004). The study of the MAUP dates back to the work of Gehlke and Biehl (1934) who found that the correlation coefficient between two aggregated spatial variables: male juvenile delinquency and median equivalent monthly rents per zone, increased with the size of analysis zones. This finding has been confirmed by different subsequent papers (Amrhein, 1995; Fotheringham and Wong, 1991; Openshaw, 1977b; Yule and Kendall, 1950).

In his study of the MAUP, Openshaw (1983) makes the distinction between the scale effect and the aggregation/shape effect. The scale effect depicts the 'variation in results that can often be obtained when data for one set of areal units are progressively aggregated into fewer and larger units for analysis'. The aggregation effect relates to the 'variation in results due to the use of alternative units of analysis when the number of units is held constant'. Both effects are arbitrary and are found to bias modeling outcomes since the definition of zones and their number is often arbitrary. Inconsistencies due to the MAUP have been raised in various econometric models: univariate analysis (Openshaw, 1979), multivariate regression (Fotheringham and Wong, 1991), gravity models (Batty and Sikdar, 1982a, 1982b, 1982c, 1982d), and discrete choice models (Guo and Bhat, 2004). Economic research in general, and urban economics in particular are still not familiar with this problem and the detrimental bias it may induce in economic analysis.

These findings seem intriguing and challenge the consistency and robustness of any spatial analysis conducted with zonal units. In this regard, Fotheringham and Rogerson (1993) declare : '[These findings] are discouraging in the sense that they make the results of any aggregate level analysis suspect and potentially unreliable.' Nonetheless, the MAUP is an intuitive finding that simply highlights a basic fact: modeling results of any spatial phenomenon $\varphi$, depend on the observational units used to capture $\varphi$ and especially on the aggregation function $G$ and the spatial division $D$ (eq. 1.1). In spatial analysis, zones are used to group individual observations and to form more tractable spatial units. Any change in the definition of these unit, their boundary, shape, or number, induces a change in modeling outcomes. In order to control for this dependency issue, literature suggests three different strategies that are detailed in the next section.

\subsubsection{Resolution strategies}

Different resolution strategies have been suggested to overcome, to reduce, or to assess the spatial aggregation bias. These strategies fall into three categories ${ }^{10}$ :

1. Error measurement.

\footnotetext{
${ }^{10}$ These resolution strategies are inspired by a previous classification made by Miller (1999).
} 
2. Optimal spatial designs.

3. Appropriate spatial methods.

The error measurement strategy consists in computing and reporting modeling errors induced by spatial aggregation. Actually, this is not a true resolution strategy in the sense that it only intends to acknowledge the extent of the aggregation bias and not to overcome it. Modeling outcomes are reported given these errors in order to assess their reliability and usefulness in decision-making. To this end, sensitivity analysis ${ }^{11}$ is often used. Different spatial designs with varying spatial resolutions are defined and feed to sensitivity analysis to characterize the variability of modeling results. In practice, few studies have been reported to use this strategy. In this line, the papers reviewed are found to adopt an experimental set-up where few, i.e. less than ten, alternative zoning systems are defined on an arbitrary basis with no regard to sampling errors that might arise from such arbitrary choices (Binetti and Ciani, 2002; Bovy and Jansen, 1983; Chang et al., 2002; Crevo, 1991; Ding, 1994; Jeon et al., 2012). In this case, no robust statistical inference can be made from such small statistical samples. Moreover, the arbitrary definition of zones in these studies is a serious source of bias that is difficult to disentangle from spatial aggregation errors.

The second resolution methodology acknowledges that modeling outcomes are unavoidably dependent on the spatial design and that this latter can be viewed as a modeling parameter (Masser and Brown, 1975; Openshaw, 1977b). This strategy views spatial division as a modeling parameter that can be calibrated in order to maximize the goodness-of-fit of the model. In this regard, the optimal zoning is a spatial division that optimizes a predefined objective function like accuracy. Practically, optimal zoning algorithms are often based on hierarchical aggregation of zones starting from a detailed zoning. Zones are progressively selected and aggregated in a manner that optimizes an objective function given some stopping criteria. One valid criticism about this strategy is that the spatial design is considered as a calibration parameter that can be used or misused to artificially boost the goodness-of-fit of spatial models. In this context, the use of an optimal zoning system can mask or cover-up for other modeling errors, like: errors in measurements, in specification, and in calibration that interfere with the construction of the optimal zoning system.

Both previous resolution strategies acknowledge that the spatial aggregation bias is due to spatial aggregation itself. The last resolution strategy, however, recognizes that the aggregation bias is rather an artifact of using inappropriate modeling methods on aggregate spatial data. If convenient spatial methods are used, one can get rid or reduce the impact of the spatial aggregation bias or reduce its extent (Ay et al., 2017; Batty and Sikdar, 1982a; Tobler, 1989). In this line, spatial modeling has long consisted in applying aspatial methods to spatial phenomena. For instance, linear regression analysis and OLS estimation are widely used in spatial analysis despite spatial autocorrelation in independent variables that violates the condition of independence of observations

\footnotetext{
${ }^{11}$ The sensitivity of a model towards a parameter refers to the impact this parameter can have on modeling results. To assess this impact, sensitivity analysis examines the elasticity of modeling outcomes with respect to the parameter.
} 
and residuals. The spatial aggregation bias is therefore more a fallacy induced by applying wrong methods to spatial data than a problem of data, per se. In this regard, different authors suggest more convenient methods to undertake spatial analysis (see Anselin (2013) for a review of spatial econometric methods).

In our effort to develop practical land-use and transport models (LUTM) and to contribute to their dissemination and use in the decision-making spheres, a need to address the question of spatial aggregation issues arises. Literature suggests that these issues can undermine the overall reliability and usefulness of modeling outcomes. Literature also suggests some resolution strategies whose contribution is still to be investigated.

Given the three years allocated to this $\mathrm{PhD}$, these questions cannot be fully addressed in all land-use and transport sub-models. The author has made the choice to limit the scope of the current research to transportation modeling. The focus on transportation modeling has two main motives:

1. Within the standard LUTI framework, four step models are still the most predominant modeling framework in use ${ }^{12}$.

2. Spatial aggregation in transportation models has received little attention from research with comparison to land-use models ${ }^{13}$.

In this context, the spatial aggregation problem in transport models can undermine the global potential of the LUTI approach to become a practical decision-aid tool. It is therefore of vital importance to understand how space is modeled in transport models and what are the implications of such modeling choices on the quality and the reliability of modeling outcomes. The answer to these questions can help define new modeling strategies that account more suitably for the spatial aggregation problem in LUTI models. To this end, the research scope of this thesis work is limited to the spatial aggregation bias in four step models.

\subsubsection{Four Step Models and the spatial aggregation bias}

Four step models are the most common transport modeling framework in both academia and urban planning. In land-use and transport interaction models, the four stage framework is often used to compute aggregate accessibility indicators. The following section succinctly presents conventional four stage models. The reader can refer to more detailed presentation in Ortúzar and Willumsen (2011) or Bonnel (2004).

\footnotetext{
${ }^{12}$ Some LUTI models are investigating the opportunity and the contribution brought by coupling a land-use model like UrbanSim (Waddell, 2002) and an agent-based transport model like MATSim (Horni et al., 2016). See the SustainCity project for more details.

${ }^{13} \mathrm{~A}$ recent research on the spatial bias in land-use models is undertaken in Jones (2016).
} 


\subsubsection{Trip generation}

The four stage modeling chain starts with trip generation. Trip generation computes for each Traffic Analysis Zone (TAZ) trip attractions and productions. Trip attractions $A_{i}$ of zone $i$, refer to total travel demand whose destination is zone $i$; trip productions $P_{i}$ of zone $i$ refer to total outgoing trips from zone $i$. To better capture these quantities, different factors are used: trip purpose (travel to work, to school, shopping, leisure, others), time of day (morning peak-hour, evening peak-hour, off-peak periods), and person characteristics (income, household structure, car ownership). Different methods are used to compute trip generation. For a comprehensive review of these methods, see Ortúzar and Willumsen (2011) and Bonnel (2004). Common methods are based on regression

analysis or cross-classification. As demonstrated by Openshaw (1979) and Fotheringham and Wong (1991), regression analysis has been shown to be subject to the spatial aggregation bias. Cross-classification methods often use spatial variables to group individual characteristics and thus, they are likely to be subject to the spatial aggregation problem as well.

Now that for every zone, trip attractions and productions are computed, the distribution stage comes to distribute these quantities between origins and destinations.

\subsubsection{Trip distribution}

The distribution stage comes after trip generation and distributes trips, i.e. attractions and productions between zones. Practically, the distribution step computes $T_{i j}$ : the number of trips between zones $i$ and $j$, using the productions of $i$ and the attractions of $j$. Various methods have been developed to perform this operation. In practice, the most used method is based on spatial gravity modeling. The principle idea behind gravity models can be derived from the utility maximization framework: when a person can undertake an activity in two different places, this person is more likely to choose the nearest place in order to minimize its transportation costs, everything else being equal. Trips are therefore distributed between zones inversely proportional to the travel cost between these zones. Other distribution methods have also been suggested: discrete choice models (McFadden, 1978), intervening opportunities (Stouffer, 1940), entropy maximization (Wilson, 1969), and radiation models (Simini et al., 2012).

In a study of optimal zoning-systems, Openshaw (1977b) demonstrates the vulnerability of gravity models to aggregation bias and especially to the MAUP. Parameter estimates of the deterrence functions are found to vary unpredictably when the spatial design varies. In another series of papers by Batty and Sikdar (Batty and Sikdar, 1982a, 1982b, 1982c, 1982d), the authors acknowledge the problem of spatial aggregation in spatial interaction models in general and in gravity models in particular, before setting a framework for this problem and suggesting a solution inspired by the information theory, i.e. entropy. 


\subsubsection{Modal split}

At the distribution stage, trips between zones are computed without assigning any travel mode to these trips. The determination of travel modes is performed at the modal split stage. For each couple of zones $i$ and $j$, trips between these zones $T_{i j}$ are split between travel modes in competition. Utility maximization using discrete choice models is the most common framework to model modal split. These models compute the probability of using a mode $m$ according to its relative utility in comparison with other travel modes. The utility function is a combination of different variables that influence individual modal choices. These variables often relate to: trip maker characteristics (car ownership, household structure, income, etc.), travel mode characteristics (travel time, cost, reliability, regularity, etc.), and journey characteristics (trip purpose, parking availability, access/egress times, etc.) (Ortúzar and Willumsen, 2011).

In a paper dealing with residential location choice models, Guo and Bhat (2004) demonstrate the vulnerability of discrete choice models and specifically of multinomial logit models toward the modifiable areal unit problem. These findings suggest that applying discrete choice models on spatially aggregated data raises questions on the reliability of modeling results.

\subsubsection{Traffic assignment}

Traffic assignment is the final step in the four stage chain. The assignment stage is where travel demand meets transport supply to compute network performance indicators, i.e. levels of service. To do so, each trip is assigned to the shortest path/route that joins its origin and destination conditional on its travel mode and network congestion levels. The computation of the shortest routes is based on the graph theory. In transport modeling, assignment modeling dates back to the work of Wardrop (1952) who stated a set of conditions under which network equilibrium appears: 'The journey times on all the routes actually used are equal, and less than those which would be experienced by a single vehicle on any unused route'. This equilibrium is known as the first principle of Wardrop or the selfish user equilibrium where each traveler minimizes its individual travel time considering congestion delays ${ }^{14}$. Thanks to advancements made in programming and operational research, these principles have been reformulated as algorithms and solved as computer programs (Beckmann et al., 1956; Dijkstra, 1959; Frank and Wolfe, 1956). Different assignment algorithms exist and the reader can refer to Patriksson (2015) for an extensive review on these models.

Traffic assignment models produce two main outcomes: link flows and travel times. To this end, different modeling choices are made to model travel demand and transport supply: definition of traffic analysis zones (TAZ), coding of transport networks, and the definition of zone connectors.

\footnotetext{
${ }^{14}$ The second principle of Wardrop known as the social equilibrium states that '[...] traffic should be arranged in congested networks in such a way that the average (or total) travel cost is minimised' (as cited in Ortúzar and Willumsen (2011)).
} 


\subsection{Research questions and contribution}

\subsubsection{Research questions}

The review of literature on the spatial aggregation bias shows that this issue has already been discussed and inconclusive attempts of resolution have been made in a variety of models and especially in:

- Discrete choice models used extensively in land-use and modal split modeling.

- Regression analysis used in trip generation.

- Gravity models used at the distribution stage.

The impact of aggregation on assignment models has received limited attention from research. Yet, traffic assignment is a key stage in transport modeling in particular, and in LUTI modeling in general. In transport modeling, traffic assignment outcomes are used to compute travel cost functions that are required by predecessor stages: trip distribution and modal choice ${ }^{15}$. In LUTI modeling, traffic assignment is essential to the computation of accessibility indicators. Any bias in assignment is then detrimental to the overall LUTI modeling given the interdependencies and feedback loops within this framework. For all these reasons, the current $\mathrm{PhD}$ focuses on assignment models to understand the extent of the spatial aggregation bias.

The spatial aggregation problem has two major implications in traffic assignment models ${ }^{16}$ :

- Loss of information on local access and egress conditions.

- Omission of intrazonal trips in assignment models.

Access and egress conditions to transportation facilities are of major importance in transport modeling. These conditions are deemed to impact main travel decisions, including: the decision to travel (trip generation), the choice of destination (trip distribution), the choice of travel mode (modal split), and the route choice (traffic assignment). In this context, the spatial aggregation problem induced by using zone centroids is a serious modeling issue since standard assignment models cannot take into account local access and egress conditions. This might bias main modeling outcomes and produce unreliable results. In the current research, we assess the extent of this bias and suggest a new modeling strategy to reduce it.

The omission of intrazonal trips is another modeling error induced by spatial aggregation. Traffic assignment models ignore intrazonal trips since these trips start and end within the same basic

\footnotetext{
${ }^{15}$ Some authors have also tried to include accessibility indicators in the trip generation step (see Ortúzar and Willumsen (2011)).

${ }^{16}$ One might also add network coding and consistency between spatial and network resolutions to this list. These issues are discussed in the current dissertation.
} 
spatial units. Consequently, assignment outcomes are biased by this omission, especially in coarse models. Errors induced by this omission are examined and their impact on modeling results assessed. In this thesis, new assignment strategies that account more explicitly for the spatial aggregation bias and the assignment of intrazonal trips are also investigated.

To address these issues: access modeling and omission of intrazonal trips, three research questions are at the heart of this thesis:

1. How space is modeled in traffic assignment models?

2. What are the implications of these modeling choices on assignment modeling results, quality, and reliability?

3. What will be the contribution of new modeling strategies that account more suitably for spatial aggregation in traffic assignment?

\subsubsection{Thesis contribution}

4 major contributions can be drawn from this research:

- Contribution 1: the traditional modeling method of centroid connectors is found to bias main transit assignment outcomes. The use of the geographic position of zone centroids instead of actual trip makers' locations has an impact on transit modeling. To generate centroid connectors, most transport models rely on two conditions: the maximum number of connectors and their maximum length. These constraints are often chosen on an arbitrary basis and are found to be a serious source of error. Chapter 2 investigates the extent of this bias and acknowledges the need for a better modeling method of connectors. Findings from this research are currently under review in a peer-reviewed journal.

- Contribution 2: a new automatic definition of centroid connectors that relies more on detailed data than the geographic position of zone centroids is found to improve main transit assignment outcomes. The use of the spatial distribution of residents and workers and their probability of using transit facilities boosts the quality of modeling results. This allows for a better reproduction of observed data including transit ridership and transfer rates. This definition is implemented as a software program in order to be applied in an automatic manner to reduce modelers' intervention and to update the definition of connectors in line with updates in spatial distribution of residents and workers or in the definition of the network. This contribution has been published as a research paper in Transportation Research Part A: Policy and Practice journal (Manout et al., 2018).

- Contribution 3: the omission of intrazonal trips in traffic assignment models has a significant impact on main modeling results. This omission induces a fictive free-flow situation where travel times, link flows, and congestion levels are underestimated and accessibility indicators 
overestimated. This situation is detrimental to the reliability of modeling outcomes. This impact varies according to road hierarchy: local streets are more impacted by this omission than highway roads. It is therefore necessary to develop new assignment strategies that account for the omission of intrazonal trips in particular and for the spatial aggregation bias in general. Findings from this paper have been published in a research paper in Transportation journal (Manout and Bonnel, 2018).

- Contribution 4: the inclusion of intrazonal trips in traffic assignment models improves the quality of modeling results. The fictive free-flow bias induced by the omission of these trips is alleviated in comparison with the traditional assignment. However this inclusion is not sufficient to counteract the spatial aggregation bias. A new spatial issue dubbed the rerouting problem arises. The rerouting problem refers to the impact of the definition of centroid connectors and especially of connection nodes on route choice modeling. This problem is, to some extent, unavoidable in traditional four step models and especially when the spatial resolution is coarse. Chapter 5 suggests a new modeling strategy to subdue this problem. Findings from this chapter are intended to be published in a peer-reviewed journal.

To what extent these contributions help answer the questions at hand is addressed in Chapter 6: General Discussion.

\subsubsection{Thesis outline}

This $\mathrm{PhD}$ dissertation is divided into two parts: the first part tackles the problem of spatial aggregation in transit models by addressing the question of centroid connectors. The second part focuses exclusively on spatial aggregation errors in traffic models, i.e. private car models.

The $\mathrm{PhD}$ dissertation is organized in 6 chapters. Each chapter addresses a specific research question in a research paper format ${ }^{17}$. Chapters $\mathbf{2}$ and $\mathbf{3}$ tackle the first spatial aggregation problem in traffic assignment: modeling network accessibility. Chapter 2 adopts an error measurement strategy (see Resolution strategies) to examine the sensitivity of transit assignment outcomes towards the definition of transit connectors. The traditional definition of transit connectors relies on zone centroids and it is likely to be prone to spatial aggregation errors. Chapter $\mathbf{3}$ builds on findings from [chapter 2] and suggests a new practical method to model transit access and egress travel times. This new method is designed to reduce the impact of spatial aggregation by using limited intrazonal data. The contribution of this method is demonstrated in a case study from Lyon. The question of centroid connectors is only considered in the case of transit models. This is because, walking times to access and egress the transit network are a major component of total

\footnotetext{
${ }^{17}$ Exception made for chapter 5, which is a study report that compounds different contributions. This chapter is relatively longer than the rest of the chapters and it is to be formatted as a journal paper as soon as possible.
} 
transit travel times. They are also deemed to influence main travel decisions including: destination choice, travel mode choice, and route choice. Moreover, transit supply is spatially constrained and so is its accessibility. This is not the case with the private car where access/egress times are less impactful than with transit.

Chapters $\mathbf{4}$ and $\mathbf{5}$ tackle the second spatial aggregation issue in traffic assignment: the omission of intrazonal trips. Chapter 4 conducts a thorough examination of the impact of ignoring intrazonal trips in conventional traffic assignment models. This chapter relies on an uncertainty modeling framework to assess the statistical bias induced by this omission and brings to light a need for new assignment strategies that should account for spatial aggregation errors. In this regard, chapter 5 investigates 6 different traffic assignment strategies to address these issues. Findings from this study are encouraging and bring forward new research questions.

Chapter 6 undertakes a global assessment of the contribution of the thesis with respect to the research questions at hand. Findings, research contributions, and research opportunities are summarized in this chapter. 


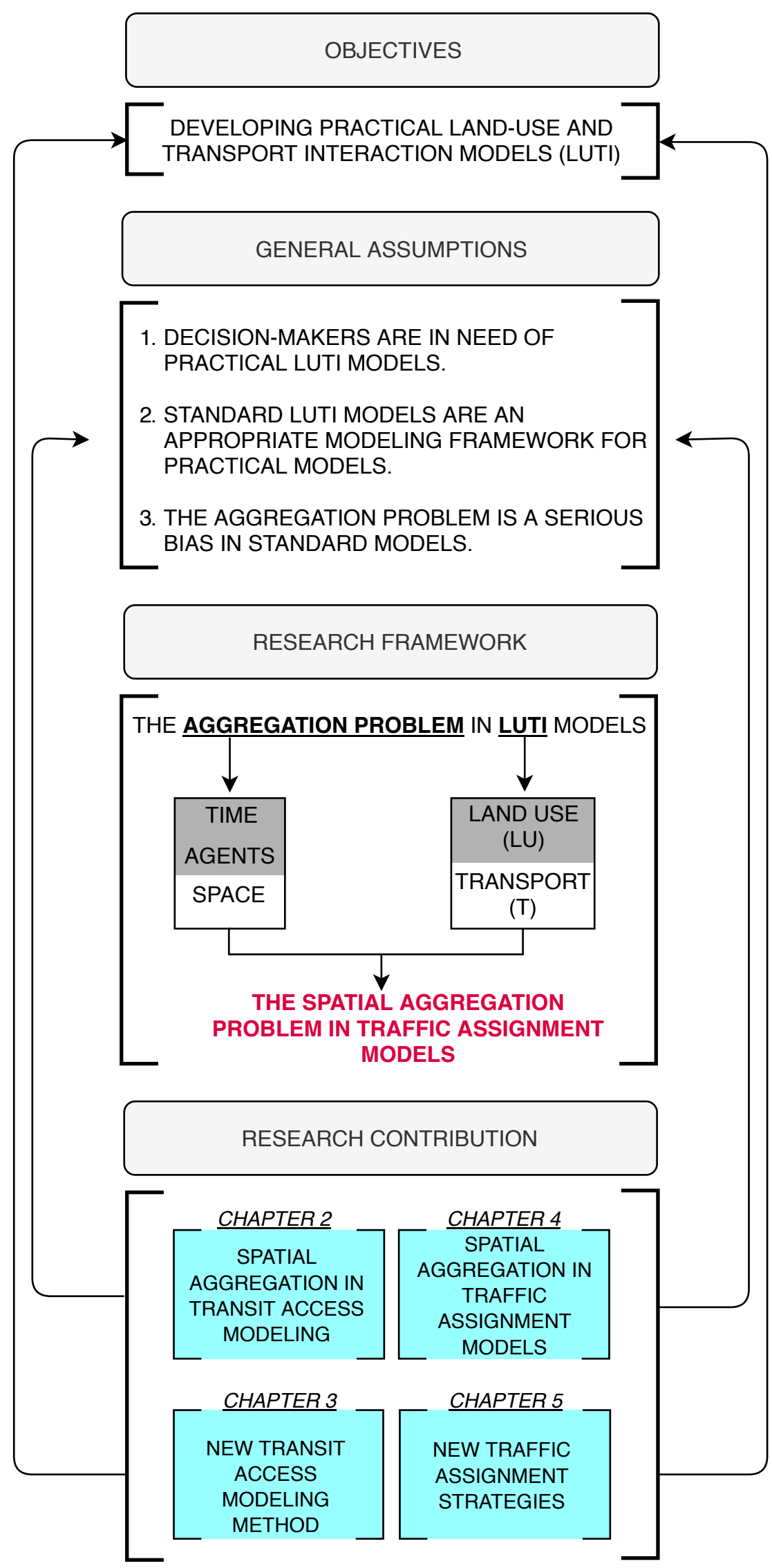

Figure 1.3: Research summary 


\section{Chapter 2}

\section{Transit access: a sensitivity analysis on the definition of transit connectors}

\subsection{Introduction}

Transit is often introduced as a sustainable and desirable alternative to car. Many cities that have long invested in car-oriented policies, are now shifting toward transit-oriented solutions. Welldesigned transit systems are believed to foster social inclusion, participation, and well-being, to contribute to economic growth and market attractiveness, and to mitigate environmental and health mobility-related issues (Delbosc and Currie, 2011; Higgins et al., 2014). In this context, cities are engaging, more or less successfully, in different transit policies to fulfill their mobility needs and to meet their sustainability objectives. One vital ingredient of success of these policies is transit accessibility, which can be defined as the ease with which transit facilities can be reached. Transit accessibility is deemed to enhance transit coverage, ridership, and attractiveness (Chowdhury et al., 2016; Redman et al., 2013). Consequently, in many cities, local authorities are making of transit accessibility a cornerstone measure in their policy agenda and substantial projects and investments are opted for (SYTRAL, 2018). Yet, when it comes to transportation models, a less emphasis is put on modeling transit access conditions. Often, transport models use simple methods based on the geographic position of zone centroids to infer walking times to and from transit facilities. These modeling methods seem to be too crude to render the importance of walking access and egress conditions as they are an essential part of the total transit journey. Walking times take part of almost all travel decisions, including: destination choice, mode choice, and route choice decisions. Increased walking times can reduce transit use and attractiveness (Alshalalfah and Shalaby, 2007; Daniels and Mulley, 2013; El-Geneidy et al., 2014; Farhan and Murray, 2006; Gutiérrez et al., 2011; Iacono et al., 2008; O'Sullivan and Morrall, 1996). In this regard, any error in modeling transit walking conditions can be detrimental to the overall modeling accuracy and reliability. Given this 
background, spatial aggregation in transit modeling is a serious source of bias.

Transport models often rely on a zonal description of space. Zones are the basic spatial units of analysis. In this context, all spatial information is summarized in one focal point dubbed the zone centroid. Consequently, all trips and activities are supposed to take place at the centroid position. In this case, access and egress to and from transit facilities are modeled using centroid connectors. Centroid connectors are fictitious network links that attach centroids to transit facilities, namely: transit stops and stations (figure 2.1). Accordingly, the standard definition of transit connectors assumes that all transit users can access transit facilities from zone centroids with no regard to their actual and individual access conditions. This is the standard practice in transportation modeling. This assumption is crude and may induce spatial aggregation errors in transit modeling.

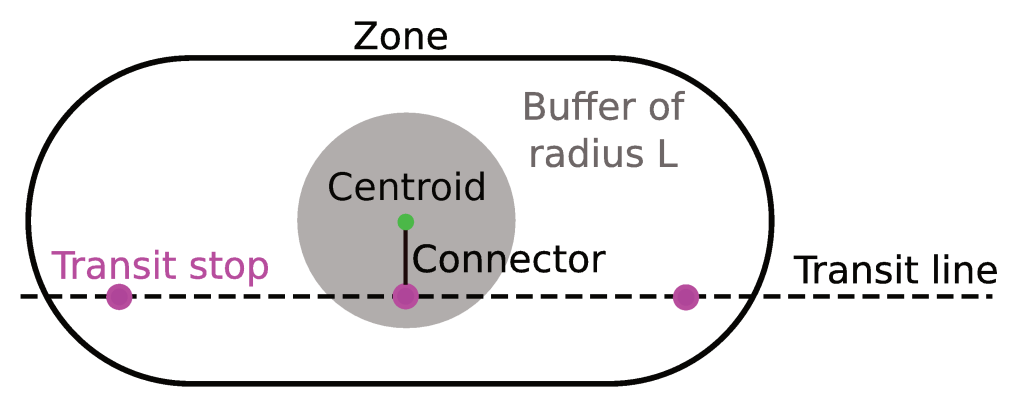

Figure 2.1: The standard definition of centroid connectors. Only the $N$ nearest stopping points within a buffer of radius $L$ around zone centroid are connected.

In the current research, the author questions the validity of this method and assumes that this definition biases transit assignment outcomes. To test the validity of these assumptions, this paper undertakes a study of modeling errors induced by the standard definition of transit connectors within a sensitivity analysis framework applied to a transit model of Lyon in France.

\subsection{Background}

The definition of centroid connectors has an impact on modeling results. This impact has already been demonstrated in the case of car connectors but it is still to be confirmed in the case of transit connectors. For car connectors, literature has shown that modeling outcomes, including link flows, are sensitive towards the definition of connectors. Different papers show a significant effect of the definition of car connectors on network flows and travel times (Chang et al., 2002; Friedrich and Galster, 2009; Jeon et al., 2012; Leurent et al., 2011; Sean Qian and Zhang, 2012).

In a comprehensive research conducted by Chang et al. (2002), the authors find out that traffic assignment outcomes are dependent on the definition of zone centroids and thus on the definition of centroid connectors. Four different configurations of centroid connectors are tested and explored in 
a statewide model of Idaho, USA. Each configuration relates to a centroid positioning method using: geometric center (configuration 1), main city center (configuration 2), population weighted center (configuration 3), and household density weighted center (configuration 4). For each configuration, an assignment of travel demand is performed using the Deterministic User Equilibrium (DUE) assignment. The transportation network and the spatial resolution are controlled for in this research by keeping them constant to isolate the effects of connectors.

Results from this research show that average travel times on the overall network vary according to the definition of centroids. Travel times decrease from configuration 1 to 4 . This is partly due to the reduction of the length of connectors when using high spatial resolution data (configurations 2, 3 and 4) rather than the widespread geometric center approach (configuration 1). Likewise, fine configurations reproduce more accurately observed network flows. In regard of these results, the authors conclude that centroid connectors can have a significant impact on assignment results and that using fine spatial data to define centroid connectors improves modeling accuracy.

In another paper, Sean Qian and Zhang (2012) demonstrate the effect of a random selection of connection nodes on traffic assignment outcomes. Connection nodes are road intersections where car trips can access and egress from the transportation network. Centroid connectors attach these nodes to zone centroids to allow travel demand to enter and exit the network. In three different case studies, the authors underline the significant impact of a random selection of connection nodes on traffic assignment outcomes. The experimental protocol of the paper consists in choosing randomly different connection nodes from the network, to construct corresponding centroid connectors, and to assign trips using these randomly chosen connectors. To this end, the Deterministic User Equilibrium (DUE) assignment is used. This protocol is applied to three different case studies: a synthetic grid network, the SR-41 corridor in Fresno, California, and the urban network of Sacramento, USA.

Results demonstrate that a random selection of connection nodes and therefore of connectors induces a significant instability in network flows and travel times. Adding more connectors does not necessarily reduce the extent of these instabilities even if this practice is largely popular among transportation modelers. At the contrary, such practice is found to cause fictitious network fluidity since unreal routes may be used to bypass the congested network leading to a decrease in total travel times. Reducing the number of connectors may also create fictitious congestion and an artificial increase in travel times. The selection of connection nodes and their number is therefore not an obvious task. The authors conclude that the definition of connectors has a statistically significant impact on modeling outcomes.

To reduce this impact, the authors suggest a heuristic selection methodology of connection nodes based on the minimization of an objective function defined as the maximum of the ratio of estimated traffic volumes to ground traffic counts. The solution to this optimization problem produces satisfactory assignment results given the predefined objective function. Nevertheless, one might argue that the definition of centroid connectors should not be regarded as an optimization problem. In our opinion, connectors are not a calibration parameter to be used or misused to calibrate 
transport models for the sake of fitting to observations. Connectors should be considered as an input information that reflects local network access and egress conditions as encountered by trip makers.

In order to account for local access conditions and their variability from one trip maker to another, Leurent et al. (2011) introduce an assignment method that allows access and egress travel times to vary between road users. This method is based on the Stochastic User Equilibrium (SUE) instead of the DUE as in previous research papers. The stochasticity introduced by this assignment framework allows for access and egress travel times to vary between road users according to a predefined statistical distribution. The choice of a route, including access and egress links, is performed using discrete choice modeling techniques (Ben-Akiva and Lerman, 1979; McFadden, 1978). Despite its theoretical attractiveness, results drawn from this new definition show a marginal contribution when compared to the standard assignment method using centroid connectors. The contribution and validation of this new definition needs further research before drawing any conclusive findings.

Another method dealing with car connectors is introduced in Friedrich and Galster (2009). The authors consider travel times of car connector as the sum of: (1) walking times between the origin and the parking slot of the private car; and (2) the in-vehicle travel time spent in the secondary network, i.e. local streets. For egress connectors, a parking search time is added to (1) and (2). Access and parking search times are considered constant and are deduced from a German survey conducted on 500 car owners. In order to determine the in-vehicle travel time spent in the secondary network, two traffic models are implemented: a macroscopic and a microscopic model. The spatial design of the microscopic model is defined as a sub-zoning of the macroscopic zoning. For each macro-zone, the time spent on the secondary network is computed as the difference between the macroscopic travel time and the mean microscopic travel times. The authors suggest also different geometric methods for choosing connection nodes. The contribution of this new definition is, however, not demonstrated in the paper and validation against ground counts is not undertaken. From a practical standpoint, this method is cumbersome as it seems to require the use and the calibration of two transport models: a macroscopic and microscopic one.

This brief review of literature outlines that assignment outcomes depend on the definition of connectors. Different authors confirm this finding in different urban contexts and suggest some methods to deal with it. Nonetheless, when it comes to transit, the definition of transit connectors has received limited attention (Tamblay et al., 2016). To our knowledge, the impact of transit connectors on assignment results has not yet been demonstrated nor evaluated. As in the case of car connectors, one might reasonably expect that the definition of transit connectors may have a significant impact on modeling outcomes.

The current research addresses this issue and assumes that the conventional definition of transit connectors impacts assignment results. To stress the validity of this assumption, the current research performs a sensitivity analysis on the definition of centroid connectors and its corresponding effects on modeling outcomes. 


\subsection{Methods}

The current research explores the impact of the standard definition of centroid connectors on transit assignment outcomes using, to some degree, a similar approach to that of Sean Qian and Zhang (2012). In their sensitivity analysis, the authors examine the effects of a random selection of connection nodes in the case of car connectors by: (1) picking randomly from 1 to 6 connection nodes; and (2) performing a traffic assignment of travel demand using corresponding connectors. In our research, we extend this sensitivity analysis to the case of transit connectors using a stochastic sensitivity approach.

In the majority of transport models, the standard definition of centroid connectors relies on two modeling constraints (figure 2.1):

- A maximum number of connection nodes, referred hereafter as $N_{\max }$.

- A maximum length of connectors, referred hereafter as $L_{\max }$.

These constraints are interdependent and are used to select a maximum number $N_{\max }$ of connection nodes that are within a radius of $L_{\max }$ around zone centroids. Often, these constraints are set on a subjective basis or, at best, on modelers' expertise. In all cases, setting $L_{\max }$ and $N_{\max }$ is often arbitrary which may produce arbitrary modeling outcomes. The current paper suggests to study the extent of the impact of an arbitrary choice of these standard constraints using a sensitivity framework.

In practice, the experimental protocol consists in:

1. Choosing different values for $L_{\max }$.

2. Randomly selecting $N_{\max }$ connection nodes.

3. Assigning transit demand using selected centroid connectors.

4. Repeating steps (2) and (3) 100 times.

5. Repeating steps from (1) to (4) for different values of $L_{\max }$ and $N_{\max }$.

In step (1), a maximum length of connectors is chosen. $L_{\max }$ should translate the maximum distance that trip makers are willing to walk in order to access/egress the transit system. This distance is, obviously, individual and context-dependent; nevertheless, literature shows that nearly $100 \%$ of trip makers walk less than 1,500 meters or 25 minutes to and from transit stops and stations (Daniels and Mulley, 2013; Dill, 2003; El-Geneidy et al., 2014; Farhan and Murray, 2006; Gutiérrez et al., 2011; Hsiao et al., 1997; O'Sullivan and Morrall, 1996; Zhao et al., 2003). In the current research, 3 different values of $L_{\max }$ are tested: 500, 1,000, and 1,500 meters.

Step (2) defines for each zone $Z$ the set $\Omega_{L_{\max }}(Z)$ of all reachable connection nodes within a predefined maximum walking distance $L_{\max }$. Depending on $L_{\max }$ and on the transit system's coverage, $\Omega_{L_{\max }}(Z)$ may contain zero or more connection nodes. The experimental protocol consists, 
afterwards, in drawing randomly $N_{\max }$ different connection nodes from $\Omega_{L_{\max }}(Z)$. If a zone $Z$ has less than $N_{\max }$ connection nodes, then all its connection nodes are selected. Each connection node is attached to the corresponding zone centroid using a centroid connector. Connectors' travel times are deduced from the Euclidean distance and a constant walking speed of 1 meter/second.

In step (3), a transit assignment is performed using the set of selected connectors and different assignment outcomes are examined.

In step (4), steps (2) and (3) are repeated 100 times. This is done in order to cover all possible configurations and also to get rid of any sampling bias of connection nodes.

In step (5), steps from (1) to (4) are repeated for different values of $L_{\max }$ and $N_{\max }$.

Since the method relies on a random selection procedure, all assignment outcomes are considered as random variables. In the current study, only total transit journeys and transfer volumes are investigated. The statistical distribution of these outcomes is explored in detail in the sequel of this paper.

\subsection{Data}

\subsubsection{Transit model of Lyon}

The sensitivity analysis is conducted on a transit model of Lyon in France. This model describes the 2017 transit system of the metropolitan area of Lyon. This study area covers nearly 59 municipalities and over 1.4 million inhabitants. The transit system of Lyon is the second most important transit system in France in terms of ridership. It carries nearly 1.7 million daily trips served by 4 subway lines, 5 light rail lines, over 130 bus corridors of which 26 are Bus Rapid Transit (BRT) lines serving over 4,000 stops disseminated over 59 municipalities (figure 2.2). The transit network is $3,448 \mathrm{~km}$ long of which $94 \%$ are bus routes (table 2.1 ). 


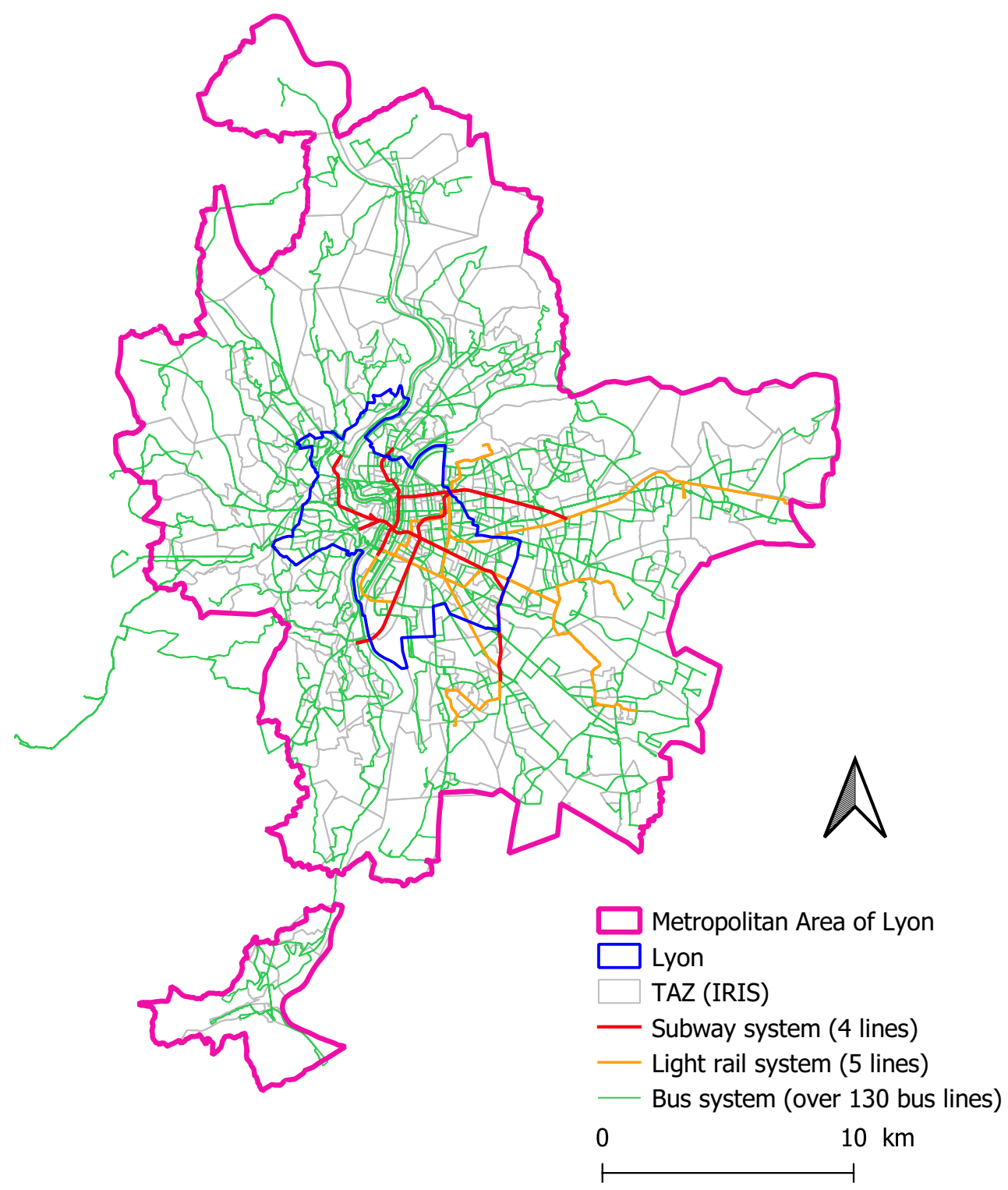

Figure 2.2: The Metropolitan Area of Lyon and its transit system

Table 2.1: Network characteristics of the Lyon transit system (GTFS, Métropole de Lyon version 2017)

\begin{tabular}{llll} 
Transit category & Number of stops & Cumulative length $(\mathrm{km})$ & $\%$ of total length \\
\hline Bus & 4,101 & 3,246 & $94 \%$ \\
Tramway & 187 & 135 & $4 \%$
\end{tabular}




\begin{tabular}{llll}
\hline Transit category & Number of stops & Cumulative length $(\mathrm{km})$ & $\%$ of total length \\
\hline Subway & 85 & 65 & $2 \%$ \\
Funicular & 5 & 2 & $0.1 \%$ \\
\hline
\end{tabular}

Transit supply is derived from the GTFS data of Lyon (GTFS, Métropole de Lyon, version 2017). The transit system is modeled in detail at the evening rush-hour (5-6pm). Each transit line and transit stop are included in the model. Onboard Travel Surveys and transit counts ${ }^{1}$ are used to compute a trip matrix of 124,041 transit journeys. The spatial design of the model is derived from the administrative IRIS subdivision (Insee, 2017). This is the most detailed official zoning available in France. Each zone (IRIS) is designed to encompass at least 2,000 inhabitants.

A deterministic frequency-based transit assignment model is used (Spiess and Florian, 1989). This approach is based on the optimization of travel strategies at each transit stop. Each travel strategy accounts for access and egress travel times, namely: connectors' travel times, onboard dwelling times, and frequency-based waiting times at stops. Travel times are weighted to translate travelers' stated preferences (table 2.2) (Abrantes and Wardman, 2011; Wardman, 2001). The optimal strategy minimizes the sum of these weighted times for each trip.

Table 2.2: Travel time factors.

\begin{tabular}{ll}
\hline Parameters & Value \\
\hline Walk time factor & 2.0 \\
Wait time factor & 2.0 \\
On board time factor & 1.0 \\
Subway service regularity & 1.0 \\
Light rail service regularity & 1.0 \\
BRT service regularity & 1.0 \\
Bus service regularity & 1.0 \\
Alighting penalty (seconds) & 480.0 \\
\hline
\end{tabular}

\subsubsection{Experimental set-up}

In the current research, the maximum walking distance $L_{\max }$ of centroid connectors is set to 500, 1,000, and 1,500 meters, respectively to 8, 16, and 25-minute walk at a speed of 1 meter/second. The $N_{\max }$ constraint is set between 1 and 100 connection nodes:

\footnotetext{
${ }^{1}$ Onboard Travel Surveys were conducted by the local transit authority between 2012 and 2016 .
} 
$N_{\max } \in[1,2,3,4,5,6,7,8,9,10,20,30,40,50,60,70,80,90,100] . N_{\max }$ increases incrementally from 1 to 10 then by 10 connectors till 100 connectors are defined. For each configuration, connection nodes are chosen randomly and so are their travel times. 5,700 simulations are run (19 configurations of $N_{\max } \times 3$ configurations of $L_{\max } \times 100$ random draws).

The current research does not compare assignment outcomes to ground counts. The main objective of this research is to study the statistical variability of transit outcomes rather than their absolute values and accuracy. In this line, the transit model of Lyon has not been finely calibrated. Calibration errors are hence assumed to be independent of the definition of connectors and that findings from this research will hold true in finely calibrated model $^{2}$.

\subsection{Results}

The main transit assignment outcomes are found to depend on the definition of transit connectors. As in the case of car connectors, findings show that both the maximum length and the number of centroid connectors have an impact on transit ridership, all modes taken together and on each transit mode.

\subsubsection{Impact of connectors on the overall transit ridership}

The standard definition of transit connectors is found to impact assignment outcomes and especially, transit ridership. In figure 2.3, predicted transit ridership, which is the sum of transit boarding and transfers, is found to depend on the maximum number and length of connectors. When the number of connectors increases, total ridership decreases. Conversely, transit ridership increases with the maximum length of connectors.

\footnotetext{
${ }^{2}$ In fact, detailed calibration of transport models often relies on "fine-tuning" the definition of connectors. In our case, this practice would mask the impact of the definition of centroid connectors on assignment outcomes.
} 


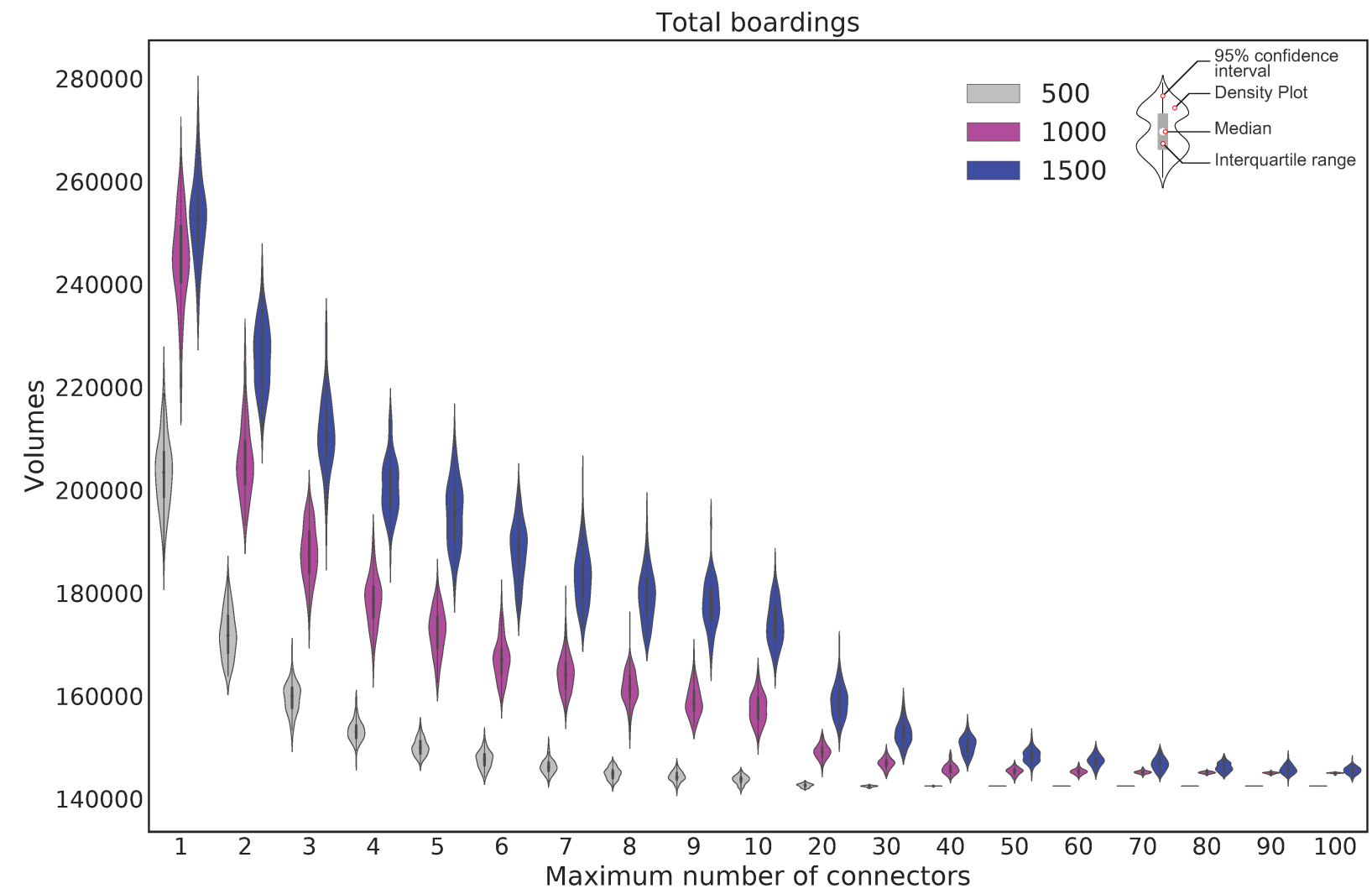

Figure 2.3: Impact of transit connectors on total transit ridership. X-axis depicts the maximum number of connectors $N_{\max }$. The Y-axis depicts corresponding transit ridership. Three values are used for $L_{\max }: 500,1,000$, and 1,500 meters

In the case of Lyon, transit ridership drops by almost 100,000 trips ${ }^{3}$ from nearly 250,000 to 150,000 trips when the maximum number of connectors increases from 1 to 100 (figure 2.3). This decline occurs despite the fact that travel demand remains constant throughout all simulations. Therefore, depending on the maximum number of connectors per zone and their maximum length, transit models can predict different values of transit ridership.

For a given value of $N_{\max }$ and $L_{\max }\left(L_{\max }=1000\right.$ and $N_{\max }=10$, for example), an arbitrary choice of connection nodes induces varying values of modeling outcomes. This variance is depicted by the violin plot (figure 2.3). In this regard, transit ridership depicts a Gaussian distribution ${ }^{4}$ with a mean $\mu$ and a standard deviation $\sigma$. This distribution is due to the 100 random draws of different connection nodes. Findings from this research suggest that the statistical characteristics of this distribution depend on $N_{\max }$ and $L_{\max }$, namely: $\mu=\mu\left(N_{\max }, L_{\max }\right), \sigma=\sigma\left(N_{\max }, L_{\max }\right)$. In other words, the impact of the standard constraints $N_{\max }$ and $L_{\max }$ on modeling outcomes is statistically

${ }^{3} \mathrm{~A}$ trip is considered here as a travel journey or movement between an origin and a destination using a specific travel mode or a combination of modes.

${ }^{4}$ The Gaussian form derives from the used Gaussian Kernel-density estimation method. 
significant.

To confirm this result, two parametric tests on equal variance (Levene's test) and equal mean (Welch's t-test) are run (M. B. Brown and Forsythe, 1974a, 1974b; Snedecor and William, 1989; Welch, 1947). The following null-hypotheses are introduced:

1. $H_{0}$ : all configurations have the same variance.

2. $H_{0}^{\prime}$ : all configurations have the same mean.

Statistical tests reject the equality hypotheses for the majority of configurations (figure 2.4). Most connector configurations have statistically different means and variances. Accordingly, the traditional definition of centroid connectors has a statistically significant impact on transit ridership. Does this impact differ between transit modes? The next section answers this question.
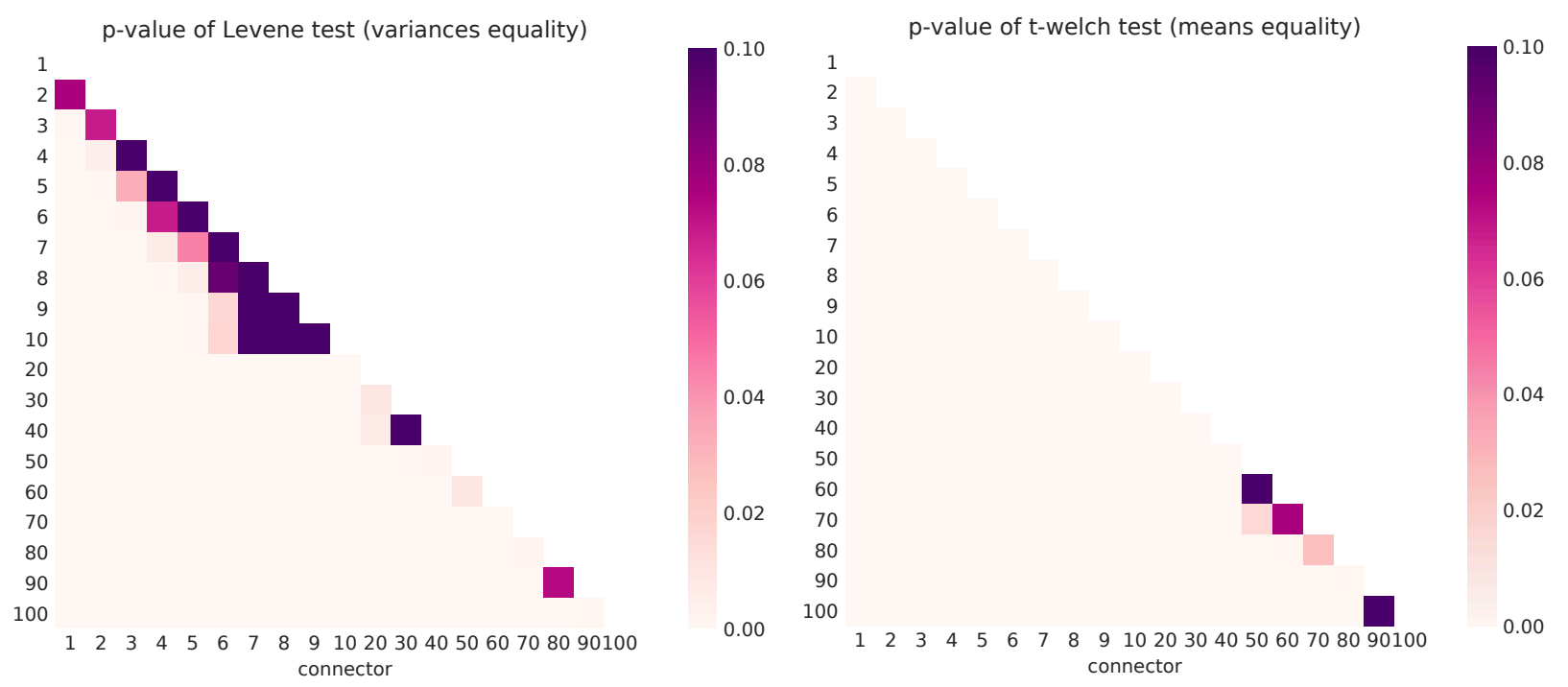

Figure 2.4: The p-value of statistical tests of $H_{0}$ and $H_{0}^{\prime}$ on mean and variance equality. $\mathrm{X}$-axis depicts the maximum number of connectors $N_{\max }$

\subsubsection{The impact of connectors on modal ridership}

On all transit modes, the definition of transit connectors is proven to have a significant impact on transit ridership. However, the extent of this impact can vary between transit modes. The subsequent analysis is carried out on each individual travel mode, namely: the bus, rapid bus, subway and light rail.

Ridership of the bus and rapid bus systems are by far the most sensitive travel modes towards the definition of transit connectors (figure 2.5). In configurations with a maximum length of 1,000 meters, average bus ridership drops from 100,000 to 45,000 trips with a standard deviation of 14,436 
trips. For the rapid bus system, ridership drops from 94,011 to 44,132 with a standard deviation of 13,780 trips. For these travel modes, the correlation between transit ridership and the number of connectors is negative. The correlation between transit ridership and the length of connectors is positive.
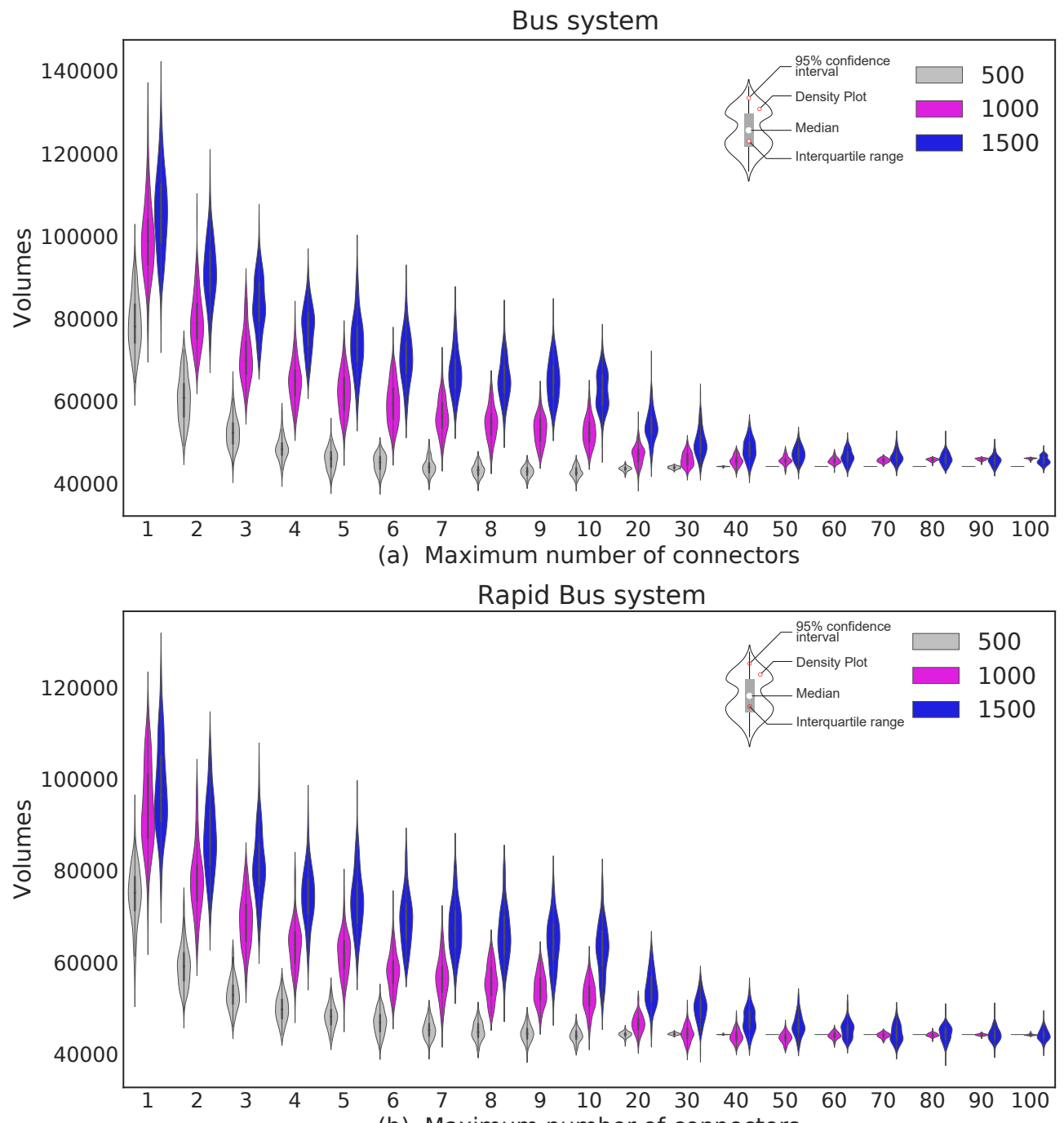

(b) Maximum number of connectors

Figure 2.5: Statistical distribution of the impact of transit connectors on total ridership of (a) bus and (b) rapid bus systems. X-axis depicts the maximum number of connectors $N_{\max }$. The Y-axis depicts corresponding transit ridership. Three values are used for $L_{\max }$ : 500, 1,000 , and 1,500 meters

For the subway and light rail systems, the impact of connectors is relatively moderate with respectively 3,688 and 930 trips as a standard deviation in the case when $L_{\max }$ is set to 1,000 meters. For both travel modes and in contrast with previous findings, the correlation is almost positive between the maximum number of connectors and transit ridership and almost negative between the 
maximum length and transit ridership (figure 2.6).

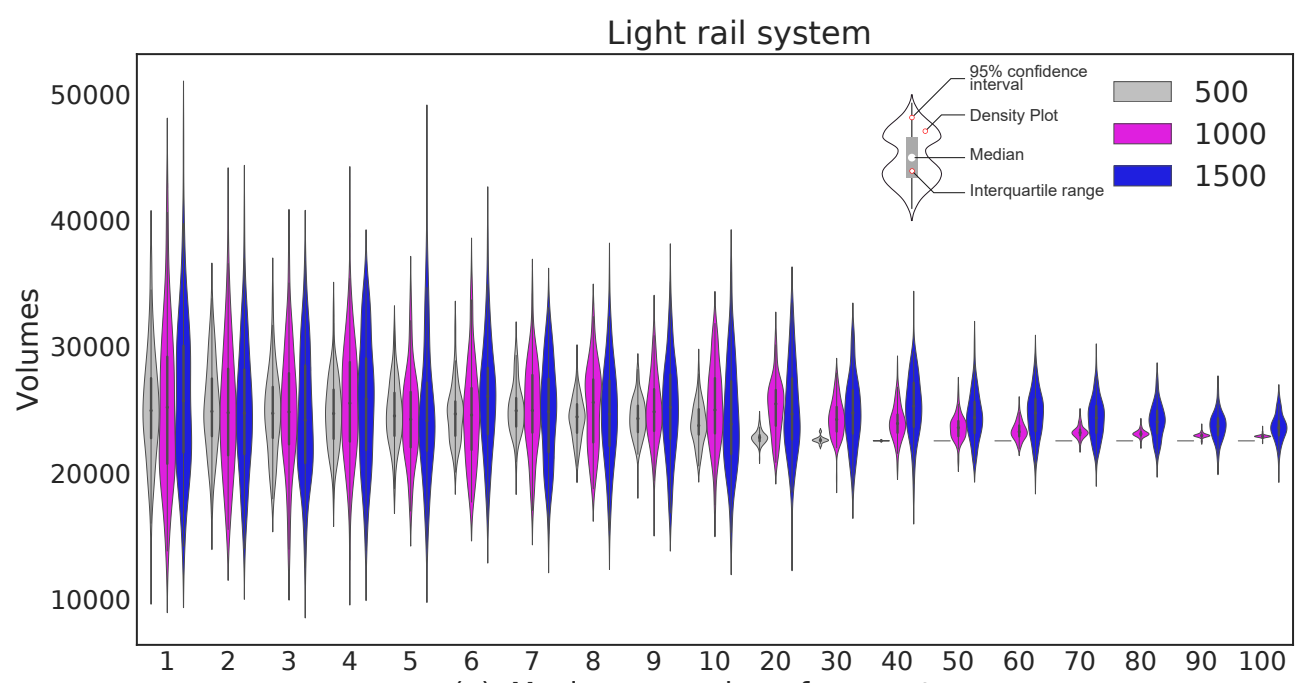

(a) Maximum number of connectors

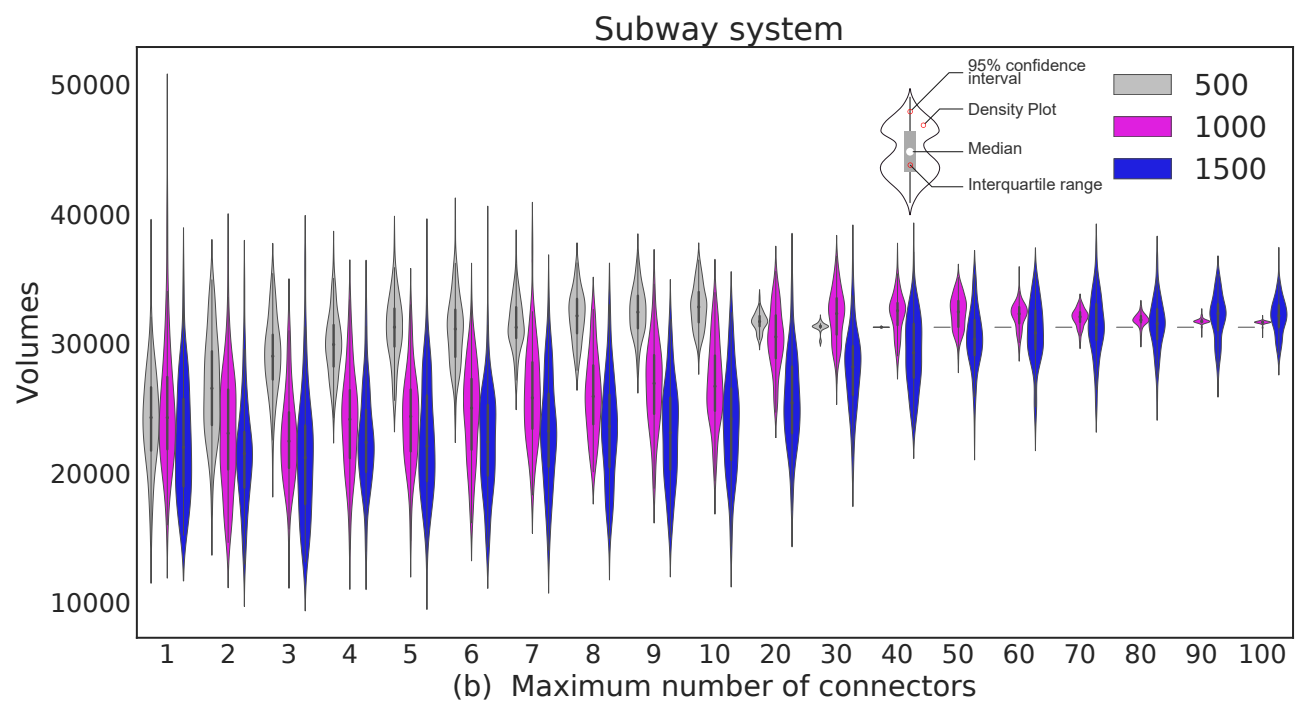

Figure 2.6: The statistical distribution of the impact of transit connectors on total ridership of (a) light rail and (b) subway systems. X-axis depicts the maximum number of connectors $N_{\max }$. The Y-axis depicts corresponding transit ridership. Three values are used for $L_{\max }$ : $500,1,000$, and 1,500 meters

Statistical tests confirm the significance of the impact on each individual travel mode. In some configurations and especially in those that do not differ substantially from each other, statistical tests do not reject the null-hypotheses of equality. 


\subsection{Discussion}

\subsubsection{Correlation between transit ridership and $N_{\max }$}

The observed decline in total transit ridership in figure 2.3 is mainly due to a decline in transit transfers (figure 2.7). When a low number of transit connectors is chosen ( $N_{\max } \leq 10$ for example), travel demand has limited access to the transit network and therefore must take different transit routes and bear several transfers to reach its final destination. When the number of connectors increases, more routes become accessible, i.e. reachable by foot within the predefined $L_{\max }$ limit. Consequently, less transit transfers are performed. This explains the observed drop in transfers as the number of connectors increases and thereby the decline of total transit ridership.

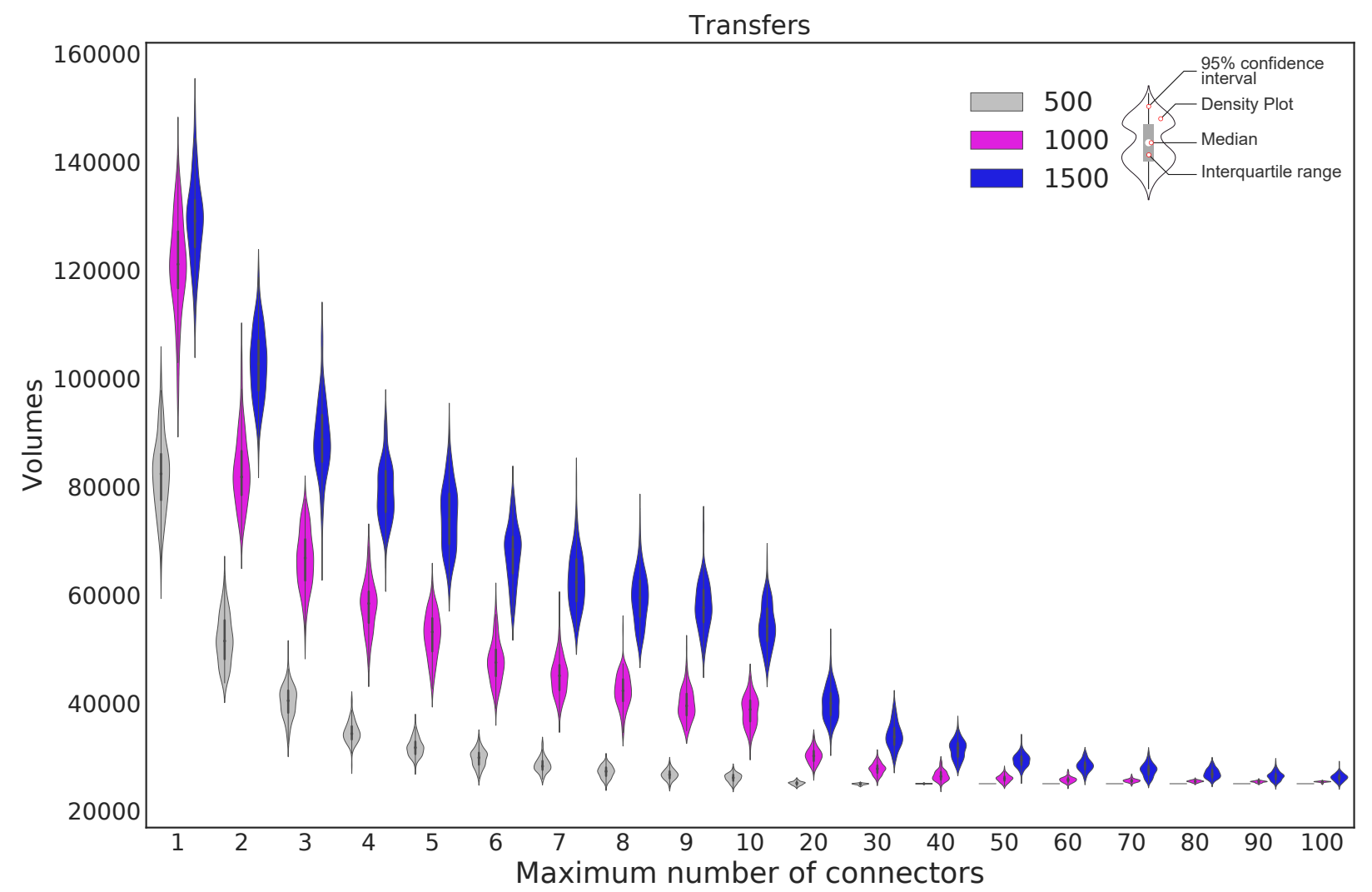

Figure 2.7: Impact of transit connectors on transfers of all transit modes. X-axis depicts the maximum number of connectors $N_{\max }$. The Y-axis depicts corresponding transit transfers. Three values are used for $L_{\max }$ : 500, 1,000, and 1,500 meters

This finding is of interest to our research. In fact, the selection of connection nodes and their number has a major impact on modeling results and especially on transfers. An arbitrary choice of these nodes is likely to produce artificial transfer trips and induce an overestimation of transit ridership. The selection of these nodes is thus a key point in the definition of connectors. 


\subsubsection{Correlation between transit ridership and $L_{\max }$}

Results show that total transit ridership increases with the maximum length of connectors. This finding holds for the bus and rapid bus systems. For the subway and light rail systems, this finding is less obvious (figures $2.3,2.5,2.6,2.7)$. When $L_{\max }$ increases, the population size of connection nodes of each zone, i.e. $\operatorname{Cardinal}\left(\Omega_{L_{\max }}(Z)\right)$, increases as well. Therefore, the probability of selecting the appropriate connection nodes that minimize the overall transit journey decreases when $L_{\max }$ increases. For this reason, configurations with higher values of $L_{\max }$ are more likely to produce higher transfer rates than other configurations since trip makers are compelled, in average, to use inappropriate connection nodes.

\subsubsection{Impact on bus and rapid bus}

Findings point out that the bus and rapid bus systems are highly sensitive towards the definition of centroid connectors with comparison to the rest of travel modes. This fact is mainly due to the design of the transit system of Lyon. The bus system, including main BRT lines, are designed to serve as neighborhood transit services. Accordingly, the spatial density of bus stops is high and covers almost all the study area (figure 2.2, table 2.1). This system is therefore easily reachable with comparison to other transit modes. Hence, when restrictive constraints are used, the standard method compels trip makers to use the bus system instead of using other means of transportation that are considered falsely unreachable by foot like the subway. Giving this situation, when $N_{\max }$ is too restrictive, ridership is overestimated for the bus and rapid bus systems and underestimated for the subway and light rail systems. Nevertheless, as $N_{\text {max }}$ increases, other means of transportation become reachable by foot (figure 2.6) causing a significant drop in bus ridership (figure 2.5).

\subsubsection{Decreasing variability of results}

As shown in figures 2.5, 2.6, and 2.7, the standard deviation of transit assignment results decreases with the number of connectors. This is an artefact of the experimental protocol. When the number of connectors to be drawn is high, $N_{\max } \geq 50$ for example, zones with less than 50 connectors use all their connectors and thereby produce similar network flows despite the 100 draw rule ${ }^{5}$. For this reason, the variability of assignment results decreases when $N_{\max }$ increases.

\footnotetext{
${ }^{5}$ In fact, for these zones, all their centroid connectors are drawn but not necessarily used. Travel demand is distributed between connectors that produce the shortest travel strategies.
} 


\subsection{Conclusion}

Findings from this research suggest that main transit assignment outcomes, namely: transit ridership and transfer rates, are dependent on the definition of transit connectors. As in the case of car connectors, the definition of transit connectors and particularly of their maximum number and length are proven to bias modeling outcomes if chosen arbitrary (Sean Qian and Zhang, 2012). An arbitrary choice of these constraints may produce arbitrary results. Setting a low value of the maximum number of connectors is likely to induce excessive transfer rates and vice versa. On the contrary, setting a high value of the maximum length of connectors is likely to produce high transfer rates on the overall transit system and especially on feeder modes like the bus.

If the extent of this impact is context-dependent, findings outline however the critical importance of the definition of centroid connectors and the potential effect they may induce if not defined properly. Yet, most academics and practitioners use the standard definition of centroid connectors. This definition relies on the geographic position of zone centroids and thereby is subject to the spatial aggregation bias. Added to this, this definition makes use of ad hoc constraints to infer access and egress conditions. In this regard, findings from this research cast serious doubts on the reliability and accuracy of transit outcomes when computed using the standard definition of connectors.

To overcome this bias, a special attention should be paid to the definition of centroid connectors. A new definition of these links that accounts more suitably for the selection of connection nodes and the computation of their length independently of the position of zone centroids is needed. Connection nodes represent access points to the transit network and their selection has a direct impact on route choice and transit use. In this regard, the selection of these nodes should, as far as possible, translate access and egress conditions as encountered by transit users. To this end, detailed intrazonal data on walking conditions and population distribution might be of interest. In the next section, a new definition of transit connectors is introduced. This definition makes use of detailed intrazonal data to overcome some of the limits of the standard definition of transit connectors. 


\section{Chapter 3}

\section{Transit accessibility: a new definition of transit connectors}

\subsection{Introduction}

Public authorities often set transit accessibility as a vital target in promoting transit use and endorsing transit policies. The improvement of transit access conditions and especially walking conditions is deemed to improve the overall quality of the transit service, the door-to-door user experience and ultimately, the transit share (Brons et al., 2009). In this context, transit accessibility or transit access refers to the ease, in terms of proximity in distance or time, with which residents and workers can reach transit facilities. There is a profuse literature on the relationship between transit use and transit access conditions (Cervero and Kockelman, 1997; Daniels and Mulley, 2013; ElGeneidy et al., 2014, Ewing and Cervero (2001); O'Sullivan and Morrall, 1996). These studies show that short access times encourage transit use and that long access times, in contrast, disadvantage transit ridership. In fact, from an economic perspective, walking to or from transit facilities is considered as a disutility associated with the consumption of the travel service. Therefore, the higher the cost, the lower the demand. Accounting for transit accessibility is therefore an important issue in modeling and appraising transit policies.

In transport modeling, however, access to transit facilities is still modelled in a very approximate manner based on centroid connectors (Ortúzar and Willumsen, 2011). Local access conditions are often overlooked and walking to and from transit facilities is simply modeled by direct links called centroid connectors. Centroid connectors attach zone centroids to transit stops and model transit access and egress by the resultant direct links. In fact, in the majority of transport models, whether they are aggregate or disaggregate, all trip origins and destinations are aggregated into traffic analysis zones (TAZ). The entire information lying inside a zone is summarized by a single point called the zone centroid. This aggregation process results in a loss of information about access and 
egress conditions. As a result, transit access is modelled by centroid connectors without considering the effects of local characteristics on walking conditions and especially on walking times. Centroid connectors often connect zone centroids to the nearest transit facilities. The corresponding walking time is, also, often directly deduced from the length of the connector. This definition, which is still widely used by both, practitioners and researchers, does not necessarily take account of the local access conditions encountered by transit users and still less of users' practices and behaviors (Bonnel, 2004). Consequently, the standard definition of centroid connectors may bias the main modeling results, as demonstrated in chapter $\mathbf{2}$ and ultimately, the policy decision.

In this paper, we shall describe a new method for modeling transit access. The method is made possible by the increasing availability of detailed spatial data. It relies on detailed estimations of walking distances to and from transit stops using fine-grained spatial data and distance decay functions. The method is also automatic and needs no major intervention from the modeler. Contrary to the standard method, the resulting transit connectors are less affected by the spatial aggregation bias and, in particular, the geographic position of zone centroids.

\subsection{Background}

While there is a plentiful literature on how transit access influences transit use (Alshalalfah and Shalaby, 2007; Cervero and Seskin, 1995; Dill, 2003; Hsiao et al., 1997; Zhao et al., 2003), it is surprisingly sparse when it comes to transposing this influence to the definition of transit access for transport modeling purposes. Few studies have addressed the problem of defining zone connectors and even fewer have dealt with the special case of transit connectors. The major literature we have found deals with private car connectors (Chang et al., 2002; Daganzo, 1980b; Friedrich and Galster, 2009; Leurent et al., 2011; Sean Qian and Zhang, 2012), while little research has focused on transit connectors (Tamblay et al., 2016). Car connectors definition has been found to impact main modeling results. In this line, transit connectors have not been directly addressed and are tacitly assumed to be similar to car connectors. Yet transit connectors differ from private car connectors in a number of respects. Unlike car connectors, the travel time on transit connectors is a significant component of the total journey travel time. Walking speeds on transit connectors are very much slower than driving speeds on car connectors. Transit connectors are also constrained and highly sensitive to the choice of connection nodes. The only possible connection nodes are the available and accessible transit stopping points. Finally, in transport modeling, walking time is often penalized since it is considered to be unpleasant by transit users (Abrantes and Wardman, 2011; Wardman, 2001). A penalty coefficient or a time multiplier, often greater than 2 , is applied to access and egress times. Consequently, transit connectors seem to have a wider impact on modeling results than car connectors.

The literature shows that both the travel times and connection nodes of car connectors have a significant impact on modeling outcomes (Chang et al., 2002; Friedrich and Galster, 2009; Sean Qian 
and Zhang, 2012). Chang et al. (2002) have shown that the zone centroid placement method and thus the travel time associated with centroid connectors impacts the assignment results. After testing different configurations of car connectors in a regional transport model, they concluded that using fine-grained data to define car connectors significantly improves the quality of the model. In another paper, Sean Qian and Zhang (2012) have investigated the impact of randomly selecting connection nodes and their number on traffic assignment results. They found that the random selection of nodes results in significantly unstable estimated traffic volumes and travel times. Defining connector travel times and connection nodes is therefore not straightforward.

The standard definition of connectors inevitably seems to have an impact on transport modeling. Since these effects are largely due to the spatial aggregation problem, some studies have set out to reduce this impact by using detailed intrazonal land-use data to infer averaged walking distances (Tamblay et al., 2016). Other studies have managed to get rid of this bias by using a continuous representation of space (Daganzo, 1980a, 1980b). Other researchers have tried to avoid the same bias by developing a totally disaggregated approach using detailed surveys where the exact origins and destinations of trips are known (Chapleau and de Cea, 1983); or to reduce the magnitude of this bias by refining the zoning system, especially during the assignment (Mann, 2002). Nowadays, microsimulation approaches can get rid, to some extent, of this spatial aggregation bias and model more suitably access and egress conditions. Agent-Based Models (ABM) are an example of these approaches where access and egress conditions of each agent are defined. However, these models are rarely implemented in real case studies to assess urban policies in operational conditions mainly because of their complexity, data requirement and incompatibility with operational constraints. Furthermore, some of these methods (ABM, in particular) require precise data located at the $\mathrm{x}-\mathrm{y}$ coordinates. This requirement is not always met regarding privacy concerns. Finally, transport models, like four step models (FSM), that rely on spatial zoning are still widely used as an urban planning tool, a modeling framework and a policy-decision aid tool. As a result of this situation, operational transport models have no alternative but to use some rules of thumb to fill this methodological gap (Cambridge Systematics and Consult, 2007; WATS, 2008). These rules are often drawn from the expertise of the practitioner with almost no scientific backing.

The objective of this paper is to fill this methodological gap by proposing a new operational modeling method. The new method overcomes the limitations of the standard definition by relying on detailed intrazonal data. The method automatically selects connection nodes and computes connectors' travel times according to the actual distance of residents and workers from transit facilities and their observed practices.

\subsection{Methods}

The new definition of transit connectors relies on a detailed description of the study area. For each TAZ, a synthetic population of residents and workers is positioned at the individual building level 
of resolution. Transit routes and stopping points are also accurately represented, as are transit timetables. Unlike the standard method based on the geographic position of centroids, the new definition generates connectors on the basis of the actual position of residents/workers and accessible transit stopping points. The resulting connectors are referred as stopping point connectors or route connectors, rather than centroid connectors.

The new definition meets several objectives and tries to overcome some of the standard method's shortcomings by:

- Generating transit connectors on the basis of actual remoteness from transit facilities.

- Generating transit connectors independently of zone centroids position.

- Reducing user intervention by automatically generating transit connectors.

- Removing the standard constraints used in operational models to generate transit connectors (maximum number and maximum length).

\subsubsection{The Algorithm}

In order to satisfy the above objectives, we have developed two methods. These:

- Select connection nodes;

- Compute connectors' length.

The new definition is implemented by the algorithm below (figure 3.1): 
For each transit route, identify all served stopping points. Transit stops shared between routes are duplicated.

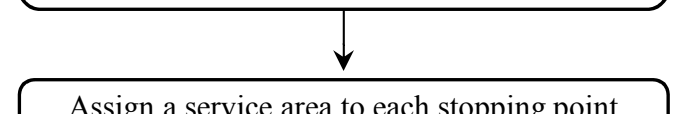

Assign a service area to each stopping point according to its corresponding transit route.

For each TAZ, identify accessible routes. A transit route is accessible if it serves a stopping point whose service area intersects a building of the TAZ.

For each TAZ and each accessible route, distribute residents and jobs between the stopping points served by this route. Residents and jobs in each building are assigned to the nearest stopping point of the accessible route.

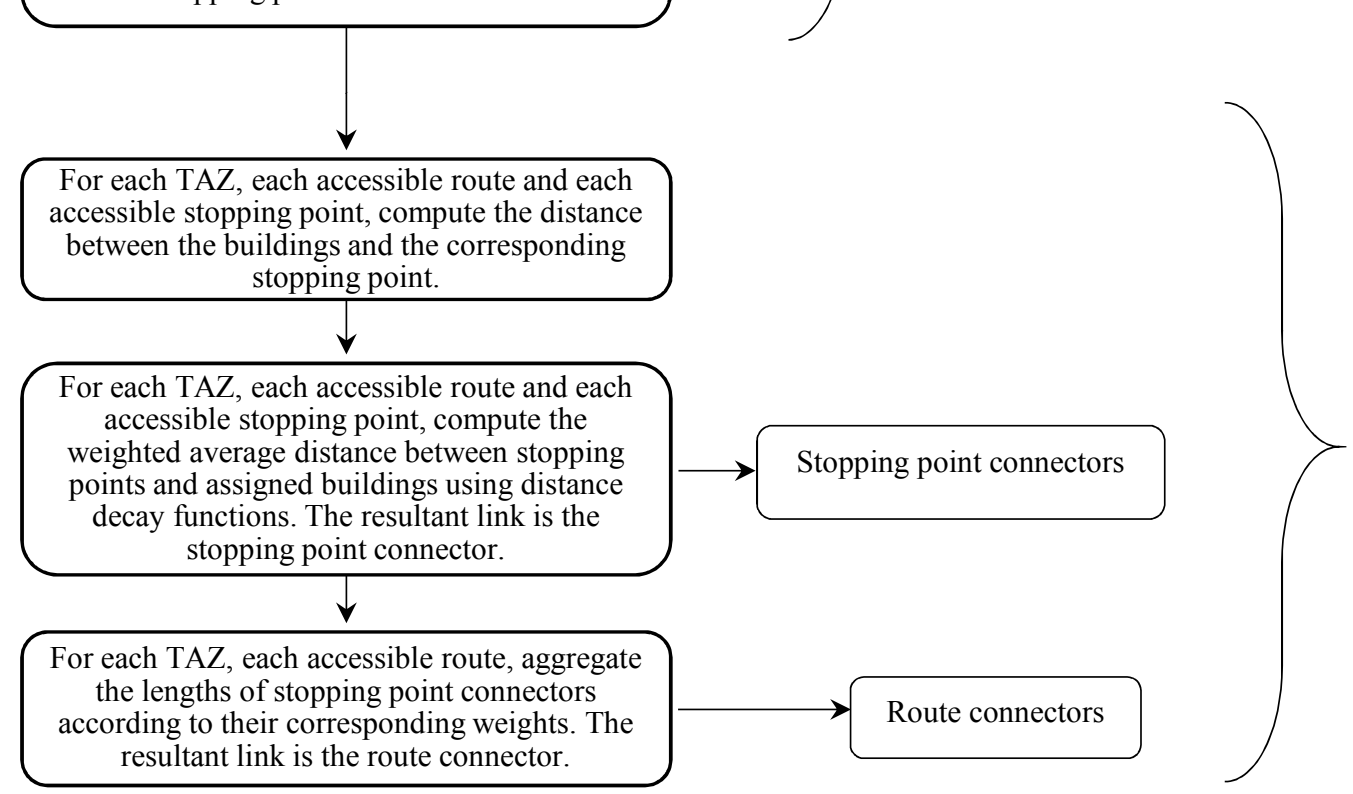

Figure 3.1: The new definition of transit connectors: the algorithm

A detailed description of each step is provided in the next section. 


\subsubsection{Justification}

\subsubsection{Selection of transit routes and connection nodes}

Unlike the standard method that constrains the number and the length of centroid connectors by imposing a maximum value, the new algorithm connects the transit routes that are reachable by the people living or working inside zones. We define the connection nodes as the set of stopping points that are accessible by foot from a zone. A stopping point is, by definition, served by only one transit route. If a stop is shared between several routes, the method duplicates this stop.

As Sean Qian and Zhang (2012) have shown in the case of car connectors, random selection of connection nodes leads to fictitious congestion or fluidity and instabilities in the model. In the case of transit connectors, random selection of connection nodes can induce artificial transfers as shown in chapter 2. Consequently, in this study, each zone is connected to all accessible transit stopping points by applying a clear methodology.

\subsection{Selection methodology}

A zone $Z$ is considered to have access to a stopping point $S_{i}$ if the catchment area of $S_{i}$ takes in at least one building or office inside $Z$ (figure 3.2). This selection method intends to include a maximum number of accessible stopping points. This number depends, of course, on the definition of the corresponding catchment area.

Let $S_{Z}$ be the set of the stopping points accessible from zone $Z$. $S_{Z}$ is defined in eq. 3.1:

$$
S_{Z}=\bigcup_{i}\left\{S_{i} \mid \min _{k}\left(d_{\left(S_{i}, b_{k}\right)}\right) \leq C_{R_{S_{i}}}\right\}
$$

$d_{\left(S_{i}, b_{k}\right)}$ is the distance between building $k$ of zone $Z$ and stopping point $S_{i} \cdot \min _{k}\left(d_{\left(S_{i}, b_{k}\right)}\right)$ is the distance between $S_{i}$ and the nearest building of $Z$ to $S_{i}$.

$C_{R_{S_{i}}}$ is the radius of the catchment area of stopping point $S_{i}$. Since a user is more likely to walk further to reach a better transit service, $C_{R_{S_{i}}}$ depends on the category of the transit route $R$ serving the stop $S_{i}$ (El-Geneidy et al., 2014; O'Sullivan and Morrall, 1996). 


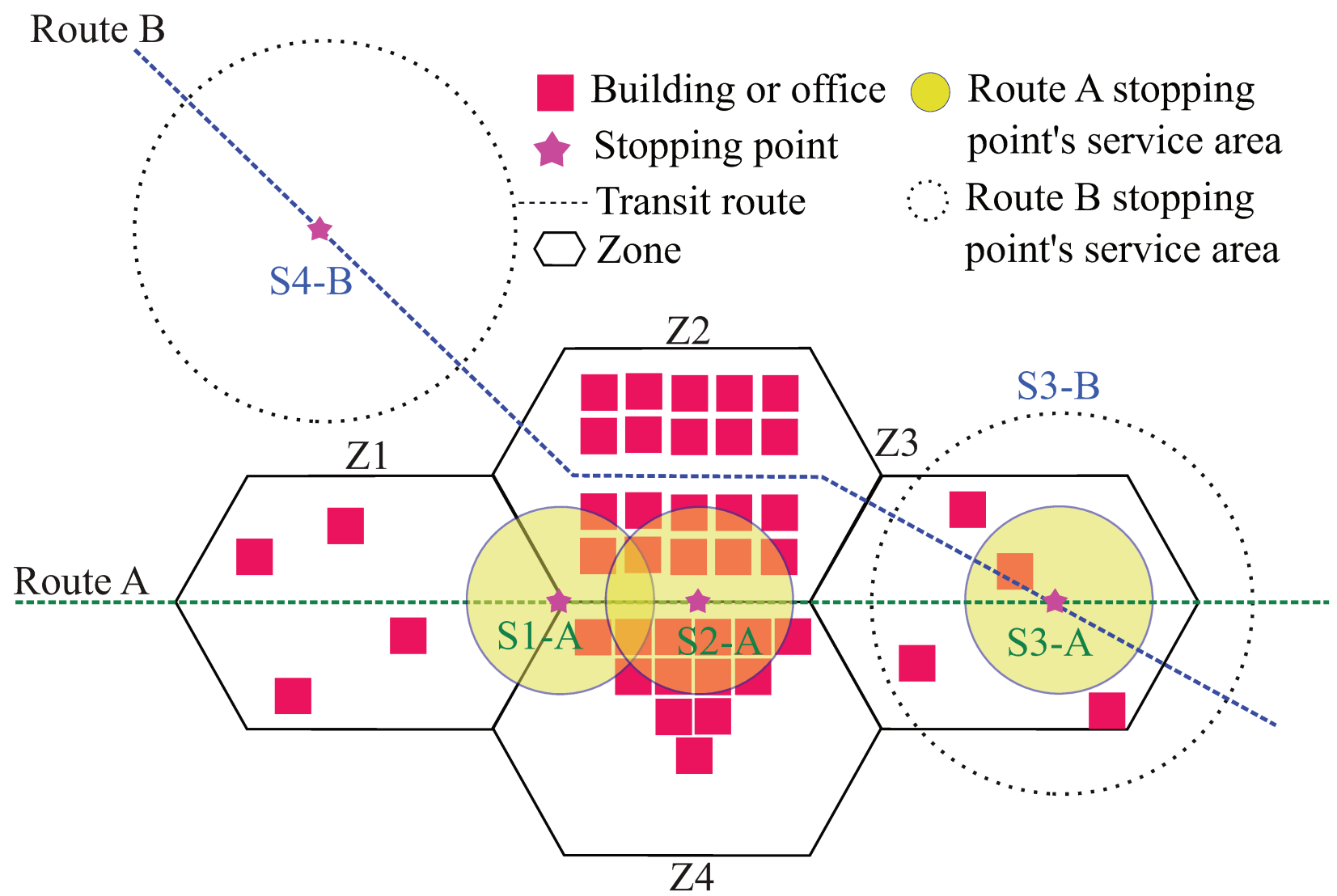

Figure 3.2: Selection of connection nodes. Transit routes A and B serve 4 transit stops and 4 zones. Route A serves stops S1-A, S2-A and S3-A. Route B serves stops S3-B and S4-B

In the example above (figure 3.2), 2 transit routes A and B (subway and bus for example) serve 4 transit stops and 4 zones. Route A serves stops S1-A, S2-A and S3-A. Route B serves stops S3-B and S4-B. The two routes share the same stopping point S3. Stopping point S3 is therefore duplicated to give two stops (S3-A and S3-B). Each duplicate has a different catchment area according to the category of its transit route.

Following the selection methodology, zone 1 has no access to any transit route. All its buildings are outside the catchment areas. Zone 2 has access to stopping points S1-A and S2-A through transit route $\mathrm{A}$. Zone 3 is served by stopping point $\mathrm{S} 3$ which is shared between routes $\mathrm{A}$ and $\mathrm{B}$. Zone 4 has access to route A through stopping points S1-A and S2-A.

For each zone, the selection procedure generates the set of all accessible transit stopping points and routes. Henceforth, each zone has a set of reachable stopping points and transit routes (table 3.1). The triplet $(\mathrm{Z}, \mathrm{R}, \mathrm{S})$ is the result of the selection procedure. 
Table 3.1: Accessible connection nodes and routes of the example in figure 3.2

\begin{tabular}{lll}
\hline Zone $($ Z) & Route (R) & Stopping point (S) \\
\hline Z2 & A & S1-A \\
Z2 & A & S2-A \\
Z3 & A & S3-A \\
Z3 & B & S3-B \\
Z4 & A & S1-A \\
Z4 & A & S2-A \\
\hline
\end{tabular}

\subsection{Distribution methodology}

Each triplet is a candidate stopping point connector. To each triplet, we assign the residents and workers that might use the corresponding stopping point. In the case where two or more stopping points share the same pair $(\mathrm{Z}, \mathrm{R})$, each building of $Z$ is assigned to the nearest stopping point. In the example in Figure 3.2, Z2 has access to route A through stops S1-A and S2-A. The population of Z2 is distributed between the two stops. As a result, for each triplet $(\mathrm{Z}, \mathrm{R}, \mathrm{S})$, a set of buildings is assigned. Here, we assign all the buildings of $\mathrm{Z}$ and not only those in the catchment areas.

The distribution procedure updates the table of triplets $(\mathrm{Z}, \mathrm{R}, \mathrm{S})$ (table 3.1$)$. Only triplets to which at least one building is assigned are kept.

\subsubsection{Connector length}

\subsection{Stopping point connectors}

The length of a transit connector must reflect the average walking distance of actual transit users and not the average walking distance of the whole population (Bonnel, 2004). The standard definition, which is based on the position of centroids, is therefore not an accurate way of measuring this length. The metric proposed here is based on the actual distance that separates potential transit users from transit facilities and their probabilities of using the transit system.

For each retained triplet $(\mathrm{Z}, \mathrm{R}, \mathrm{S})$ in table 3.1 , the length of the stopping point connector $L_{\left(S_{i}, Z\right)}$ is computed as follows (eq. 3.2):

$$
L_{\left(S_{i}, Z\right)}=\sum_{k=1}^{m} d_{\left(b_{k}, S_{i}\right)} \times \frac{f\left(d_{\left(b_{k}, S_{i}\right)}\right) \times W_{b_{k}}}{\sum_{j=1}^{m} f\left(d_{\left(b_{j}, S_{i}\right)}\right) \times W_{b_{j}}}
$$

$d_{\left(b_{k}, S_{i}\right)}$ is the distance between building $b_{k}$ and the assigned stopping point $S_{i}$. As we have already seen, each retained stopping point has a set of assigned buildings. This metric reflects the access/egress distance to/from transit facilities of potential transit users located at this building. 
$W_{b_{k}}$ is a weight associated with building $b_{k}$. This weight depends on the building usage: residential, grocery, industrial, offices, university, etc. In fact, several authors have shown a positive correlation between population and workers density around a transit station and its trip attractions and productions (Badoe and Miller, 2000; Cardozo et al., 2012; Cervero and Kockelman, 1997; Frank and Pivo, 1994; Gutiérrez et al., 2011; Kuby et al., 2004; Parsons Brinckerhoff Quade \& Douglas, 1993). Ideally, each building usage must have a specific weight corresponding to its specific potential of trip productions and attractions. For residential occupancies, this weight is the total number of residents of the building. For non-residential occupancies, the weight is inferred from the number of workers categorized by the activity sector of their firms. In this sense, different trip attractors/producers are included in the new methodology but only from a job point of view and not a usage point of view. Commercial buildings, hospitals, educational buildings are considered as trip attractors or producers proportionally to their workforce. In the case of mixed-use buildings, the corresponding weight is the sum of the different weights related to each usage (eq. 3.3).

$$
W_{b_{k}}=\alpha \times \operatorname{Residents}_{b_{k}}+\sum_{t=1}^{N} \beta_{t} \times \text { Workers }_{\left(\text {Activity_Sector }_{t}, b_{k}\right)}
$$

In equation 3.3, building $b_{k}$ is of mixed-use (residential and activity). It hosts residents and firms belonging to $N$ activity sectors. $\alpha$ weights the potential of residents relatively to workers and $\beta_{t}$ weights the relative potential between workers according to their activity sector $t$.

$f\left(d_{\left(b_{k}, S_{i}\right)}\right)$ is a distance-decay function (DDF). It expresses the effect of distance on transit ridership. A person who lives or works near a station is more likely to use transit than one who does not. A number of surveys have shown that transit use decreases when the walking distance for potential users increases (Daniels and Mulley, 2013; Dill, 2003; El-Geneidy et al., 2014; Farhan and Murray, 2006; Gutiérrez et al., 2011; Hsiao et al., 1997; O'Sullivan and Morrall, 1996; Zhao et al., 2003). The rate of decrease depends on the quality of the transit service. A good service (subway, for example) will have a larger service area whose influence will decrease gradually. El-Geneidy et al. (2014) have shown that $90 \%$ of Montréal's transit users walk less than $800 \mathrm{~m}$ to a bus stop and less than $1.2 \mathrm{~km}$ to a subway station.

For each triplet $(\mathrm{Z}, \mathrm{R}, \mathrm{S})$ retained by the selection procedure, a stopping point connector is computed. The length of the resulting stopping point connectors is deduced from the distribution of residents and workers and their probability of using the transit service.

For some well-served zones (CBD for example), this method may generate a great number of connectors per zone. These connectors can be aggregated into route connectors.

\subsection{Route connectors}

Instead of distributing transit demand between stopping points that are served by the same route, it is possible to aggregate stopping point connectors into route connectors. In this manner, each 
zone is attached to only one accessible stopping point per route. This is the case for example for the triplets (Z2,A,S1-A) and (Z2,A,S2-A) that share the same zone and the same route (table 3.1). Route connectors can be a useful way of reducing the number of generated stopping point connectors. The length of the resultant route connector is a weighted average of the length of the stopping point connectors.

Let $R_{S_{i}}$ be the transit route serving stopping point $S_{i}$ and $L_{R_{S_{i}}, Z}$ the route connector linking zone $Z$ to route $R_{S_{i}}$ through one of its reachable stopping points. The length of $L_{R_{S_{i}}, Z}$ is computed as follows (eq. 3.4):

$$
L_{R_{S_{i}}, Z}=\sum_{k=1}^{n} \frac{W_{S_{k}} \times L_{\left(S_{k}, Z\right)}}{\sum_{j=1}^{n} W_{S_{j}}}
$$

Where $S_{k} \mid k \in 1, n$ is the set of the stopping points that share the same transit route $R_{S_{i}}$ and are reachable from the same zone $Z$.

$W_{S_{k}}$ is the weight of stopping point $S_{k}$. It is the sum of the weighted buildings assigned to stopping point $S_{k}$ (eq. 3.5).

$$
W_{S_{k}}=\sum_{j} W_{\left(b_{j}, S_{k}\right)} \times f\left(d_{\left(b_{j}, S_{k}\right)}\right)
$$

In the next section, we shall evaluate the contribution of the new definition of transit connectors in the case study of Lyon in France. This definition will be compared to the standard method and to field counts. The results show that the new definition improves the main modeling results.

\subsection{A case study of Lyon}

The new definition of transit connectors has been implemented in the SIMBAD LUTI model of the Urban Area of Lyon (UAL) (figure 3.3) (Nicolas et al., 2009). In population terms, the UAL is the second largest urban area in France. Its transit system handles nearly 1.7 million trips per day. There are 4 subway lines, 5 light railway lines, over 130 bus routes and 2 funicular lines serving over 4000 transit stops spread over 59 municipalities. 


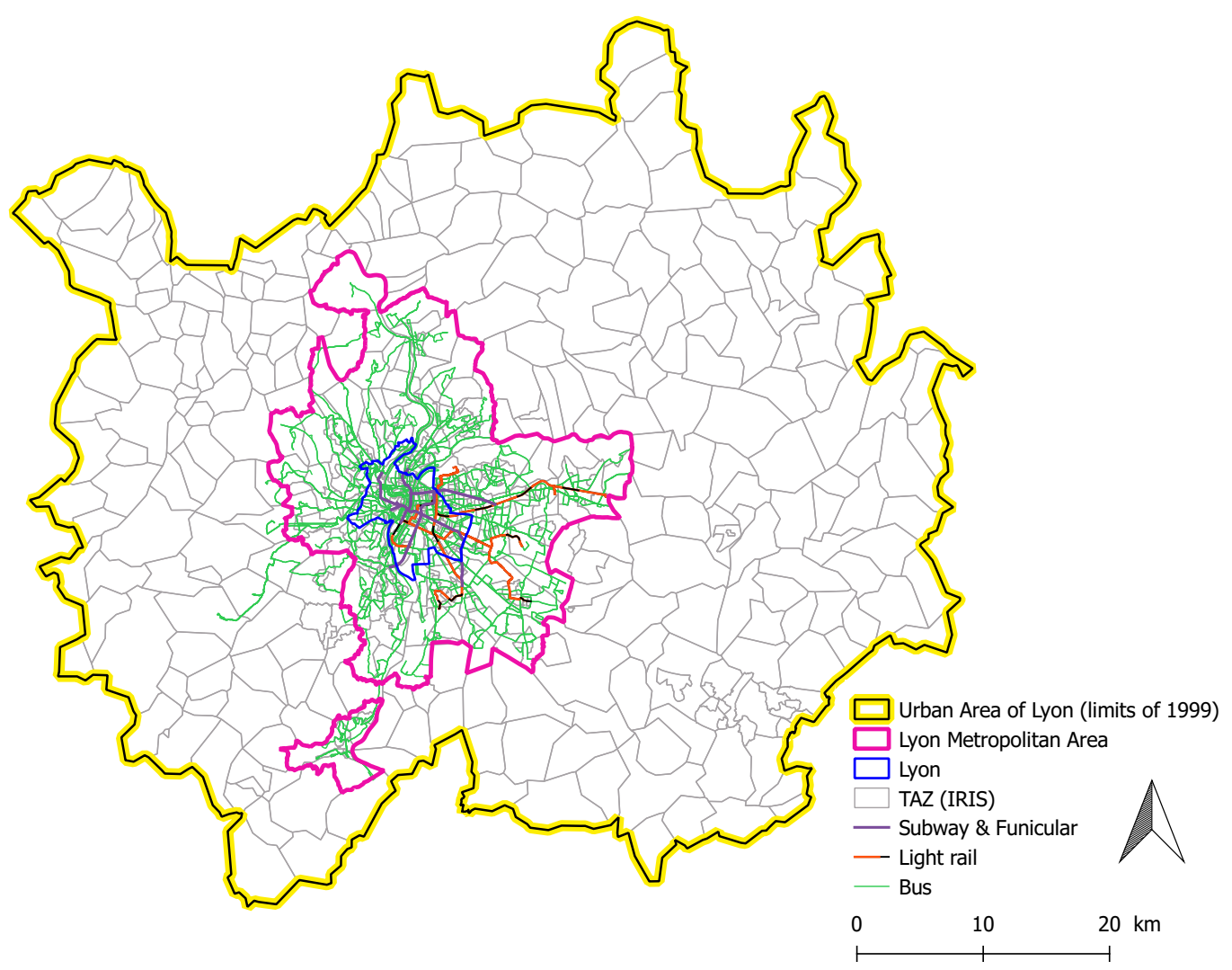

Figure 3.3: The Urban Area of Lyon and its transit system (limits of 1999) (Sources IGN, Métropole de Lyon)

To characterize the spatial distribution of potential transit users, a new dataset describing synthetic residents and workers at the building level has been prepared (Manout, 2014). To do so, 4 data sources have been combined:

- Population census dataset (Insee, 2013): population census available at the French statistical zoning IRIS (figure 3.3).

- Firms dataset (Insee, 2011): the number of workers at the building resolution is inferred from the SIRENE ${ }^{\circledR}$ declarative database of firms.

- Household tax dataset: published at a grid cell level of 200 by 200 meters. This provides the number of residents of each taxable household of the grid cell.

- BD Topo ${ }^{\circledR}$ (IGN, 2008): a GIS database describing the characteristics of the buildings (location, height, area).

The residents inferred from the household tax data are assigned to buildings according to the available housing supply. The housing supply is roughly inferred from the available living space of 
each building computed from BD Topo ${ }^{\circledR}$. To correct for disaggregation errors, a weighting procedure is performed to bring total residents of each IRIS to the census counts. Located jobs are drawn directly from the SIRENE ${ }^{\circledR}$ declarative database. For each firm, the database contains the number of employees at the office/establishment level of resolution.

The 2015 transit network of Lyon is described using the General Transit Feed Specification (GTFS) (Métropole de Lyon, 2015). Transit demand for the morning peak period (7-9AM) is deduced from onboard surveys ${ }^{1}$ and assigned to the transit network using stopping point connectors.

Due to data unavailability in the case of Lyon, distance decay functions have been calibrated using the Paris Household Travel Survey (figure 3.4) (STIF et al., 2010). Transit users from Paris and Lyon are assumed to cope similarly regarding transit access and egress. The calibrated functions have the ubiquitous distance-decay shape as demonstrated in the case of other cities from different countries (El-Geneidy et al., 2014; Hsiao et al., 1997).

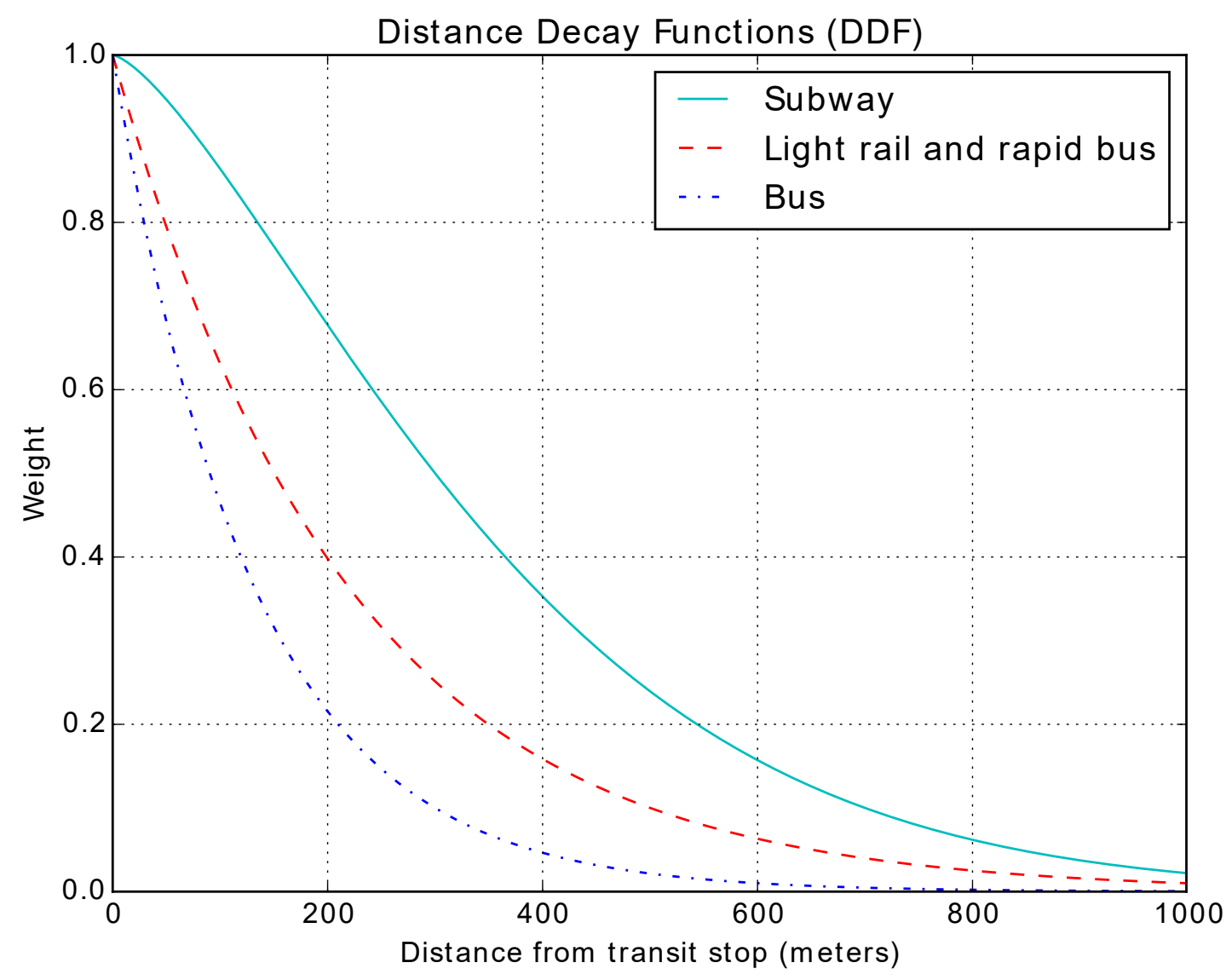

Figure 3.4: Distance decay functions by transit mode (Source: RATP data)

\footnotetext{
${ }^{1}$ Onboard Travel Surveys were conducted by the local transit authority between 2012 and 2016 .
} 
In the current case study, the weighting parameters $\alpha$ and $\beta$ are supposed equal (eq. 3.6):

$$
\alpha=\beta_{t}, \text { for all t }
$$

Residential and activity buildings are assumed to have the same potential of trip attraction/production and no difference is operated between different activity sectors. These activity sectors are assigned the same weight. This assumption is controversial in a sense that, for the same number of workers, a commercial activity is likely to attract more transit users than a back-office activity, for example. Nevertheless, we prefer not to assign arbitrary weights to different sectors. If more data are available, this assumption can be relaxed as suggested by equation 3.3.

In this case, $W_{b_{k}}$ becomes proportional to total population and workers of building $b_{k}$.

\subsubsection{Validation protocol}

The new definition of transit connectors is compared to the standard definition using observed data. The standard method defines centroid connectors as direct links attaching zone centroids to the nearest stopping points. In practice, transport models constrain connectors by a maximum number $\left(N_{\max }\right)$ and a maximum length $\left(L_{\max }\right)$.

Controlling for $N_{\max }$ and $L_{\max }$ values, several configurations of the standard definition have been tested (table 3.2). The maximum number of connectors was set at 5, 10 then 99 and the maximum length to $1,000 \mathrm{~m}$ and $1,500 \mathrm{~m}$. These configurations are henceforth named 5_1000 (for 5 connectors within 1,000 m), 5_1500 (for 5 connectors within 1,500 m), 10_1000 (for 10 connectors within 1,000 m), 10_1500 (for 10 connectors within 1,500 m), 99_1000 (for 99 connectors within 1,000 m) and 99_1500 (for 99 connectors within 1,500 m). The two latter configurations with 99 connectors intend to relax the $N$ constraint of the standard definition. The 1,500 m configurations intend to relax the $L$ constraint related to walking distances.

As expected, the number of generated connectors differs between configurations. The most unconstrained standard definitions with 99 connectors produce the highest number of connectors $(84,348$ and 55,360$)$ when compared to constrained configurations with 5 connectors $(5,194)$ or the new definition $(8,506)$.

Table 3.2: Experimental design

\begin{tabular}{ccrr}
\hline $\begin{array}{c}\text { Configuration (short } \\
\text { name) }\end{array}$ & $\begin{array}{r}\text { Maximum number of } \\
\text { connectors }\end{array}$ & $\begin{array}{r}\text { Maximum length } \\
\text { (meters) }\end{array}$ & $\begin{array}{r}\text { Total number of } \\
\text { connectors }\end{array}$ \\
\hline $5 \_1000$ & 5 & 1,000 & 5,194 \\
$5 \_1500$ & 5 & 1,500 & 5,302 \\
$10 \_1000$ & 10 & 1,000 & 10,222 \\
$10 \_1500$ & 10 & 1,500 & 10,530
\end{tabular}




\begin{tabular}{ccrr}
\hline $\begin{array}{c}\text { Configuration (short } \\
\text { name) }\end{array}$ & $\begin{array}{c}\text { Maximum number of } \\
\text { connectors }\end{array}$ & $\begin{array}{r}\text { Maximum length } \\
\text { (meters) }\end{array}$ & $\begin{array}{r}\text { Total number of } \\
\text { connectors }\end{array}$ \\
\hline $99 \_1000$ & 99 & 1,000 & 55,360 \\
$99 \_1500$ & 99 & 1,500 & 84,348 \\
The new definition & Unconstrained & Unconstrained & 8,506 \\
\hline
\end{tabular}

Transit demand of the morning peak period (7-9AM) is assigned using a timetable transit assignment procedure (Friedrich et al., 2001). Assignment results are compared to onboard counts using the following indicators: Total Transit Journeys by mode (TTJ), Transfer Rate (TR), Percent Root Mean Square Error (PRMSE) of transit volumes (eq. 3.7), and the Ratio between Observed and Estimated Passenger Volumes of transit routes (ROEPV).

$$
\left\{\begin{array}{l}
R M S E=\sqrt{\frac{\sum_{i}^{n}\left(O b s_{i}-S i m_{i}\right)^{2}}{n}} \\
P R M S E=\frac{R M S E}{\frac{\sum_{i}^{n} O b s_{i}}{n}}
\end{array}\right.
$$

The PRMSE indicator is computed for each transit mode since it is only valid when data of the same magnitude are compared.

\subsubsection{Results and discussion}

Two main conclusions can be drawn from the results. First, the main assignment outcomes depend on the definition of transit connectors. This result corroborates findings from chapter 2. Second, the new definition of transit connectors reproduces the observed data better than the standard definition. 


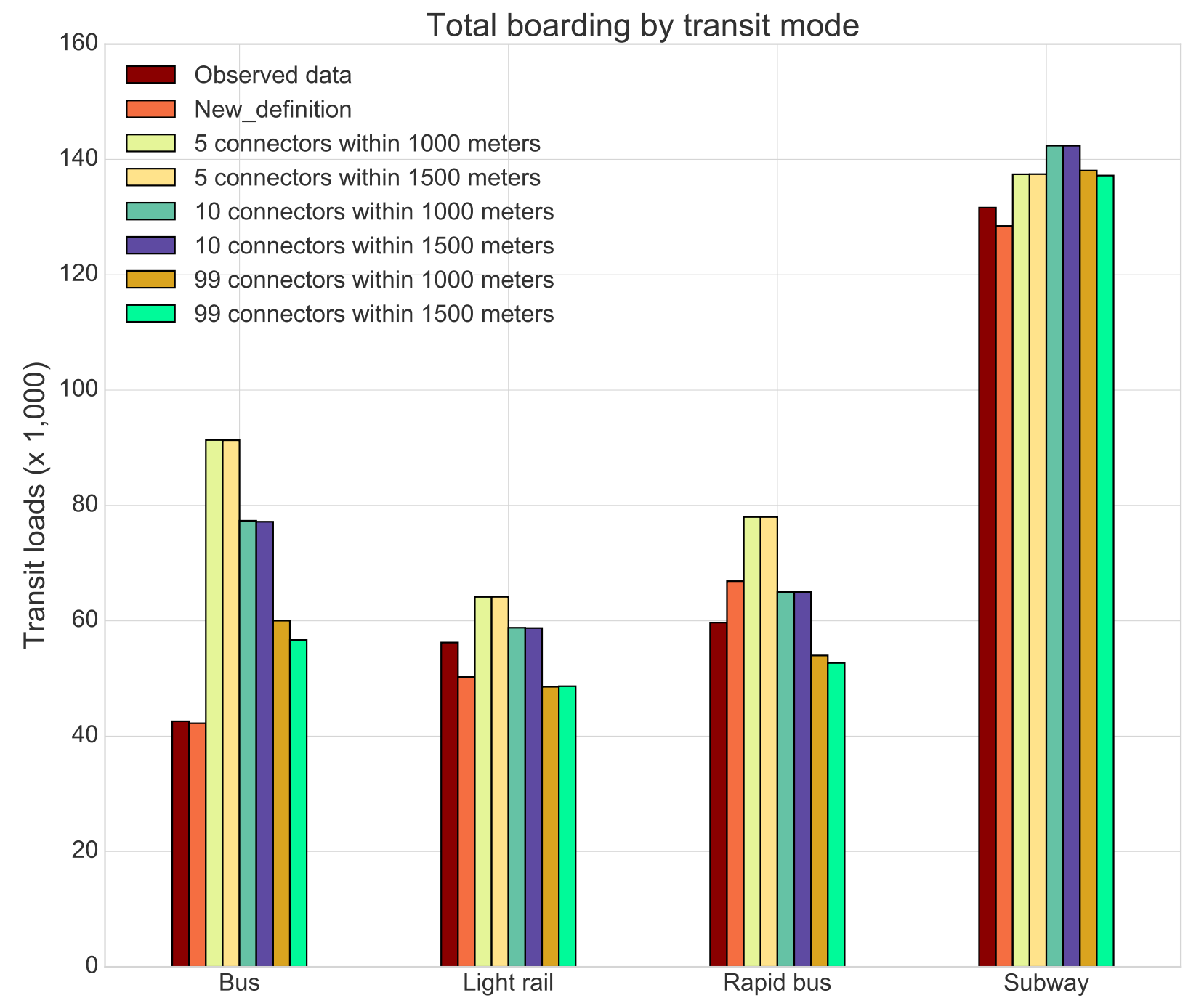

Figure 3.5: Observed and estimated total transit journeys by mode. The bars are displayed in the order of the legend 


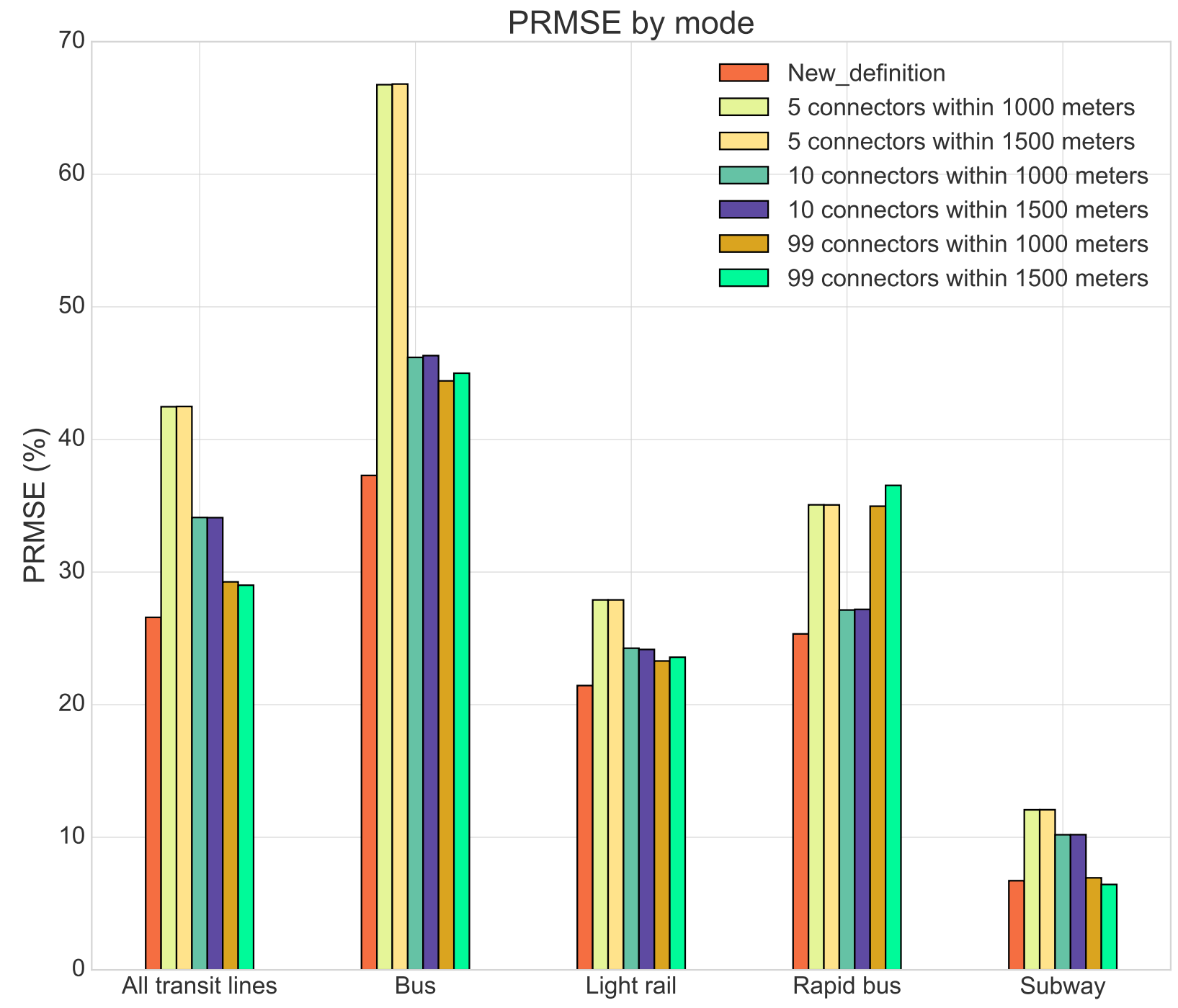

Figure 3.6: PRMSE between predicted and observed transit ridership by mode. The bars are displayed in the order of the legend 


\section{Ratio between Observed and Estimated Passenger Volumes (ROEPV)}

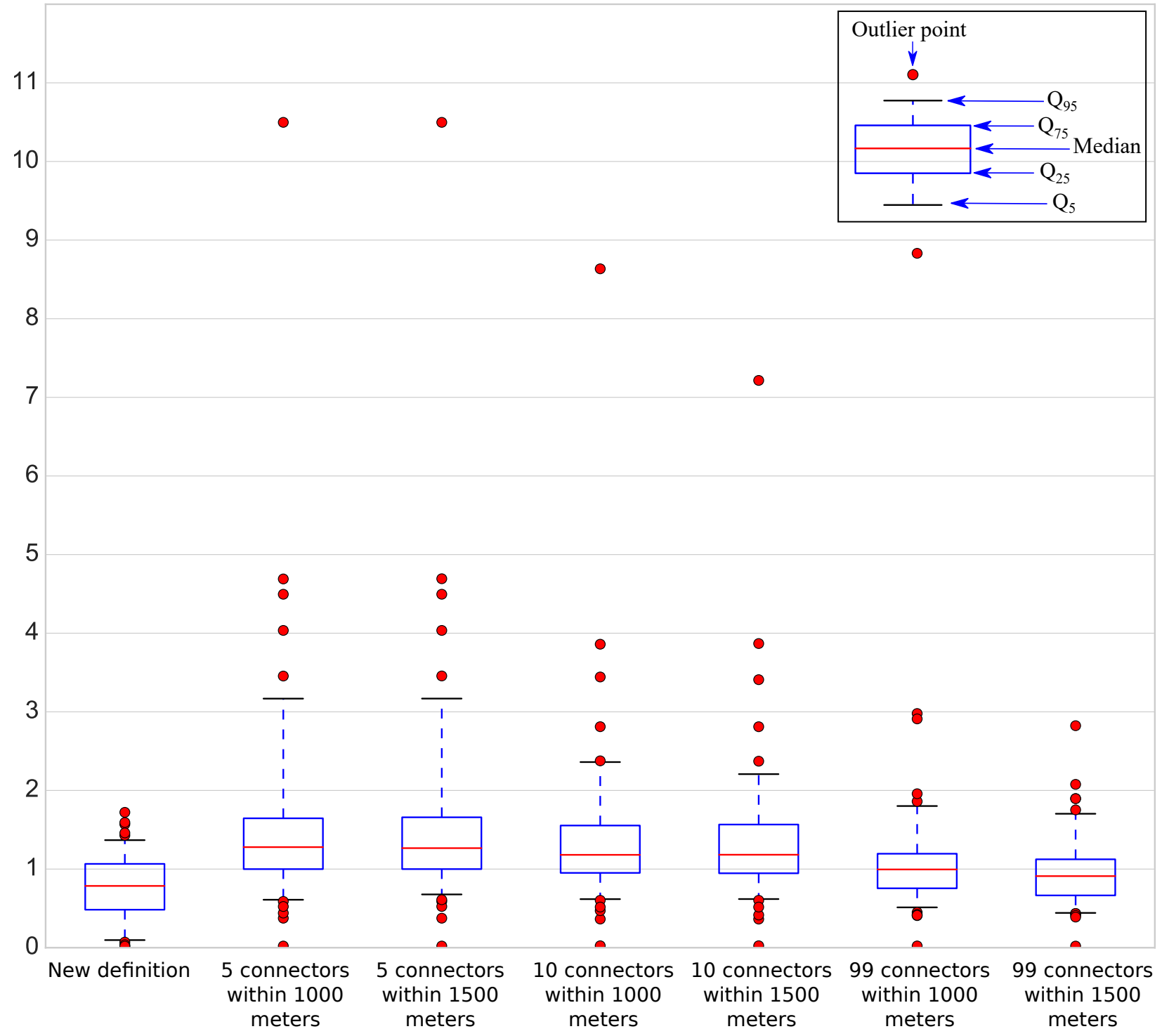

Figure 3.7: The ratio between observed and estimated passenger volumes (ROEPV)

Table 3.3: Transfer rate of the different configurations

\begin{tabular}{lllllllll}
\hline Observed data & New definition & $5 \_1000$ & $5 \_1500$ & $10 \_1000$ & $10 \_1500$ & 99_1000 & 99_1500 \\
\hline 1.37 & 1.36 & 1.74 & 1.74 & 1.62 & 1.62 & 1.42 & 1.39 \\
\hline
\end{tabular}

\subsubsection{The impact of transit connectors}

As demonstrated by chapter 2, main transit assignment results are affected by the definition of transit connectors. Estimated transit volumes and transfer rate have been found to vary significantly 
from one configuration to another (figure 3.5, table 3.3). For the bus system, for example, 42,584 trips were observed while the number of estimated trips varied between 42,239 and 91,339 . The extent of this variation differs from one transit mode to another, but it is still significant for all modes.

The results also show that the bus system is by far the mode that is the most sensitive to the definition of connectors. As stressed before, the Lyon bus system was designed as a transit feeder mode. Hence, it is easily reachable. When restrictive constraints are used, the standard method forces transit demand to use the bus system as opposed to other means of transport that are wrongly considered inaccessible. For this reason, the standard method assigns too many trips to the bus system. On the other hand, the use of access constraints may eliminate some useful direct routes and forces transit users to make more detours and use different transit routes to reach their destination. For this reason, the standard definition systematically overestimates transit use.

The PRMSE indicator confirms this conclusion (figure 3.6). The relative error varies significantly between the different configurations and transit modes. Less restricted configurations have been found to generate fewer errors than more restricted ones.

The ratio of observed to estimated passenger volumes (ROEPV) has been used to evaluate the contribution of the new method. In the case of a good fit, the ROEPV is tightly distributed around 1. For the standard configurations with 5 and 10 connectors, nearly $75 \%$ of the ROEPV distribution is greater than 1 (figure 3.7). The standard distributions also have a large number of outlier points. The unconstrained configurations with 99 connectors as well as the new definition, have a tight distribution of the ROEPV indicator that is finely distributed around 1.

The same conclusion applies to the Transfer Rate (TR) (table 3.3). The standard definition overestimates the TR indicator. As in the case of estimated passenger volumes, the overestimation of TR stems from the detours induced by the application of the standard constraints.

Finally, when controlling for the standard constraints $\left(N_{\max }\right.$ and $\left.L_{\max }\right)$, the assignment results seem to be more sensitive to the definition of $N_{\max }$ (the maximum number of connectors) than to $L_{\max }$ (the maximum length of connectors). For the same $N_{\max }$, setting $L_{\max }$ at $1,000 \mathrm{~m}$ or $1,500 \mathrm{~m}$ yielded to very close results. In contrary, the results were different when $N_{\max }$ varied from 5 to 10 then to 99 (figure 3.5, table 3.3). In fact, the increase in the value of $L_{\text {max }}$, generates few additional connectors (table 3.2). These additional links mainly attach zones that are not well served by transit and hence have little transit demand.

The findings show that the accuracy of modeling results improves as the standard constraints are relaxed, especially the $N_{\max }$ constraint. Among the standard configurations, the 99_1500 configuration reproduces more accurately observed data followed by the configurations with 10 connectors, i.e. 10_1500 and 10_1000, and finally the configurations with 5 connectors, i.e. 5_1500 and 5_1000. 


\subsubsection{The contribution of the new definition}

With the new definition, the PRMSE indicator is lower for all transit modes taken together and for each individual mode, except for the subway system. This method reduces the relative estimation errors of passenger volumes and reproduces observed data more accurately (figure 3.5, figure 3.7).

The ROEPV indicator of the new definition has a tight distribution around 0.8 and $70 \%$ of the distribution lies between 0.5 and 1.4 (figure 3.7). It also has fewer outlier points. When compared to the standard method, the new definition of transit connectors has also been found to reproduce the observed transfer rate (TR) more accurately (table 3.3). In addition, estimation errors generated by the new method are tightly distributed around zero with the lowest standard deviation of all the distributions. In contrast to the standard definition that overestimates passenger volumes, the new method seems to underestimate passenger volumes. The corresponding error density is slightly skewed to the left.

When compared to the most unconstrained standard configuration, i.e. 99_1500, the new definition produces better results. Transit boarding, and transfer rate have less estimation errors with the new definition. That is being said, the $99 \_1500$ configuration yields some interesting results that converge, in some cases, toward the outcomes of the new method. This convergence comes at the price of 84,348 connectors compared to 8,506 connectors of the new definition and longer computation times: 65 minutes compared to 45 minutes $^{2}$. In other cases, the 99 _1500 configuration produced less accurate results, especially in the case of rapid bus system. This means that the number of connectors $\left(N_{\max }\right)$ is not the only factor that guarantees good results. The way these connectors are attached to the network is also of a matter.

\subsection{Conclusion: policy implications}

In this paper, we have investigated the contribution of a new definition of transit connectors. Transit access is widely recognized as a key factor in promoting transit use and endorsing transit policies. Yet, when it comes to assess these policies within transport models relying on zones, transit access is still modelled in an approximate manner with limited consideration for local access conditions. To fill this gap, two automatic methods have been developed: (1) a procedure for selecting accessible stopping points and routes; (2) a procedure for computing the length of transit connectors. The new definition is based on a detailed description of local access conditions and potential transit users. Walking to and from transit facilities is modeled in detail using the spatial distribution of residents and workers located at the individual building level of resolution and their probability of using transit (distance decay functions). Thus, the resulting connectors take account of the relative remoteness of potential transit users and their willingness to walk in order to reach a transit

\footnotetext{
${ }^{2}$ Using a computer with a Xenon E3-1220 v5 processor, 4 cores, 3 Ghz of frequency, and 32Go of RAM.
} 
facility. In this manner, the generated connectors are independent of the geographic position of zone centroids and hence reduce the impact of the spatial aggregation problem. They are referred to as stopping point connectors or route connectors as opposed to centroid connectors.

The results shed light on the shortcomings of the standard definition. This definition is found to bias the main modeling outcomes. Therefore, when used to assess transit policies, the standard definition of transit connectors may undermine the reliability of modeling results and mislead decision-makers. This type of inaccuracy may contribute to the well-known problem of demand forecast errors or modeling errors to which the Cost-Benefit-Analysis (CBA) is particularly sensitive (Quinet, 2010; Salling and Leleur, 2015; World Bank, 2005).

In the present paper, we have developed a new definition of transit connectors that models more accurately transit accessibility and minimizes the corresponding modeling errors. The implementation of this new method in the case study of Lyon improves the main modeling outcomes. As suggested by the literature (Chang et al., 2002), we found that using detailed spatial data to characterize local access conditions boosts modeling accuracy. From a policy perspective, the new method can enhance the robustness of transport project appraisal by partly controlling for modeling errors.

The new method can also be of interest for transit policies dealing with the improvement of transit accessibility, the design of a new transit network, or the restructuring of an existing one. The design of a transit system is always subject to various conflicting criteria such as the maximization of transit coverage, against the minimization of capital and operating expenses (CAPEX and OPEX). This optimization problem has been addressed by the literature as the Transit Network Design Problem (TNDP) (Baaj and Mahmassani, 1995; Enrique Fernández L. et al., 2008) or as Stop Location Problem (SLP) (Ibeas et al., 2010; Schöbel et al., 2009). This problem refers to the question of geographically designing transit routes or locating transit stops in a manner that maximizes an objective function like social utility or local operator's utility. In this context, the new modeling method can contribute to this question by accounting for the specific impacts of locating a stop or a station in a specific area given its near environment (residential buildings, schools, hospitals...) and its reaction (distance decay function). Hence, in an automatic manner, the new method allows different stop location scenarios to be evaluated and compared as well as their wider impacts on transit use.

This being said, the standard definition is still widely used by academics and practitioners since it is simple and easy to implement. To address this issue, we have developed a computer program to automatize the implementation of the new method. The program is straightforward and needs no major intervention from the user. It is open source and freely available to both academics and practitioners, in order to overcome the limitations of the standard definition and to better assess transit policies.

Since the new method computes automatically transit connectors, it is therefore much easier to update a transit model using up-to-date census data and GTFS files with no need to calibrate the 
model or the network parameters apart from the DDF. The method allows to adjust the transit network to any change in the transport supply or in land-use patterns. Furthermore, in our case study, we used total population and jobs at the building level of resolution to compute transit connectors. If no data are available at this level, data can be located at any intrazonal level of resolution (parcels, blocks, for example).

Finally, the present method could benefit from further research regarding the distance decay functions (DDF) and the use of the network distance. As suggested by some authors (Gutiérrez et al., 2011), workers and residents do not have the same willingness to reach transit facilities. The distance decay functions for the two groups differ and need to be calibrated separately. In our case study, the same DDF have been used for both categories. It is also interesting to test the sensitivity of the model to the definition of DDF and to the definition of service areas. Additionally, we have assumed that all building usages have the same weight in attracting and producing trips. This assumption is more than questionable and needs to be relaxed by using different weights. Finally, the use of network distance in the computation of the stopping point connectors instead of the direct distance may be a way of improving the current method. The network distance is by nature more realistic and takes into account natural and artificial barriers (highways, rivers...) that may affect transit access.

\section{Acknowledgments}

The authors are especially thankful to the three anonymous reviewers of this paper. Their comments and reviews have improved the final version of the paper.

This research has been carried out thanks to the support of ForCity, Keolis, and RATP. Their support is gratefully acknowledged.

An earlier version of this paper has been published in Transportation Research Part A: Policy and Practice under DOI: 10.1016/j.tra.2018.03.028. 


\section{Chapter 4}

\section{The impact of ignoring intrazonal trips in traffic assignment models: a stochastic approach}

\subsection{Introduction}

In urban planning, transportation models are key tools in the urban planner's toolkit. These models have proven to be useful in many respects, from strategic transport planning schemes to operational traffic management operations. The outcomes of transportation models are often used to compare alternative planning scenarios and to design efficient urban policies. In this context, models have become unavoidable and even compulsory in the instruction of policy makers. In this regard, the accuracy and the reliability of these models is of importance. Inaccurate models may lead to inaccurate decisions and hence to inefficient projects bearing unpredictable costs (Flyvbjerg et al., 2005; Skamris and Flyvbjerg, 1997). Various research projects have therefore put a great focus on the design of more sophisticated models pushing forward the modeling resolution and fidelity with which urban phenomena are modeled. Nevertheless, today's transport models and their practical implementations are still prone to bias.

The spatial aggregation problem is one of these long-standing modeling errors. This problem arises from an inherent characteristic of standard transportation models: the use of Traffic Analysis Zones (TAZ). Operational transportation models often rely on spatial division to model space. Zones are the basic spatial units and all phenomena and in particular travel demand, are described using this level of resolution. In this context, only interzonal trips are included in traffic assignment models. Intrazonal trips that start and end at the same zone are unavoidably ignored as they are confined within the basic spatial units. Though, the motorized part of these trips uses the transport network, takes up some of its capacity and consequently impacts its level of service. Transport models ignore 
this impact by omitting intrazonal trips from the assignment step. This deliberate omission can therefore bias main transport modeling outcomes. The extent of this bias and its significance have long been assumed ignorable and are not yet characterized (Bhatta and Larsen, 2011).

Intrazonal trips are short journeys taking place within zone limits. If short journeys account for little total mileage, these trips may account for a non-negligible share of total motorized trips. In the urban area of Lyon for example, $25 \%$ of all car trips has a journey distance of less than $2 \mathrm{~km}$ (Agence d'Urbanisme de Lyon, 2016). Ignoring this traffic in assignment models may therefore have a detrimental impact on modeling outcomes. In fact, assigning only the interzonal part of the trip table amounts to deliberately eliminating a part of travel demand. This omission is not random

since intrazonal trips have shorter travel distances than average trips. Consequently, modeling outcomes are likely to be statistically biased by this deliberate omission.

Since intrazonal trips are dependent on the spatial design, the precise effect of their omission has long been considered as a side issue of the Modifiable Areal Unit Problem (MAUP) (Baass, 1981; Batty, 1974; Crevo, 1991; Ding, 1994; Martínez et al., 2009; Masser and Brown, 1975; Openshaw, 1983, 1978, 1977b; O'Neill, 1991). The MAUP relates to the dependency between modeling results and the modifiable nature of spatial units, i.e. their shape and number. Different modeling outcomes can be drawn from a model by using different spatial units. Nevertheless, little research has addressed the question of quantifying the specific impact of ignoring intrazonal trips in assignment models. This precise impact is currently neither demonstrated nor characterized. The extent of this impact and its significance are still to be studied. In this paper, we propose to address this issue by answering two questions:

1. Do intrazonal trips have an impact on static traffic assignment models?

2. If they do, to what extent intrazonal trips alter assignment outcomes?

In this research paper, we shall consider the specific impact of ignoring intrazonal trips in a static traffic assignment model. This research employs an uncertainty analysis framework and an experimental protocol which disentangles the effects of ignoring intrazonal trips from other related effects. This method has been developed and applied to the urban area of Lyon in France.

\subsection{Background}

Intrazonal trips have received little attention from research. From a modeling perspective, the main papers dealing with this issue can be roughly divided into two categories: (1) intrazonal trips characterization in terms of distance and travel times (Batty, 1976; Bureau of Public Roads, 1964; Kordi et al., 2012; Plaza and da Silva, 2015; Rodriguez-Bachiller, 1983; Venigalla et al., 1999); (2) the impact of intrazonal trips on modeling results (Bhatta and Larsen, 2011; Martínez et al., 2009). Since intrazonal trips cannot be characterized using traffic assignment models, research has 
developed alternative methods to infer some of their characteristics like travel times and journey distances. These methods are widely used in spatial interaction models (Batty, 1976) and rely mainly on either geometric approaches (Kordi et al., 2012; Plaza and da Silva, 2015; Rodriguez-Bachiller, 1983) or spatial interaction approaches (Batty, 1976; Bureau of Public Roads, 1964; Venigalla et al., 1999). Despite their characterization, the impact of ignoring intrazonal trips in traffic assignment models is still persistent.

Scarce research has addressed the question of ignoring intrazonal trips in transportation models, in general, and traffic assignment models in particular. In this regard, this impact has always been seen as a marginal issue in comparison with other questions like zonal or network aggregation issues (Baass, 1981; Binetti and Ciani, 2002; Bovy and Jansen, 1983; Chang et al., 2002; Crevo, 1991; Ding, 1998, 1994; Jeon et al., 2012; Martínez et al., 2009; O’Neill, 1991; Walker, 2014; You et al., 1998).

In an empirical research conducted in Eindhoven in the Netherlands, Bovy and Jansen (1983) quantify the impact of spatial and network aggregation on a urban transport model. Three different spatial divisions of the study area are defined with 1286, 183 and 47 zones. For each zoning a transport network is defined. The assignment of travel demand in the three models shows that the detailed model reproduces observations more accurately than the other two spatial representations. However, the impact of intrazonal trips is not separated from the effects resulting from the definition of the transport network or that of centroid connectors. In addition, the three models generate few intrazonal trips as short journeys are mainly made by bicycle in Eindhoven. The authors conclude that the observed effects are independent of intrazonal trips (Bovy and Jansen, 1983).

In order to show how spatial aggregation impacts assignment results, Ding (1998) studies the sensitivity of a traffic assignment model towards spatial design. The transport model is applied to South Korea, at a national level. A number of geographical divisions are defined using an automatic aggregation method. 10 different zonings are constructed by applying different aggregation criteria. The travel demand for each zoning is then assigned to the transport network that is maintained constant for the 10 models. Findings from this research show that assignment outcomes are significantly dependent on the zoning design. In this respect, Ding concludes that this impact is due to intrazonal trips that are ignored by his model. However, the precise impact of intrazonal trips is not examined nor its contribution to the observed impact.

In a research which bears similarities to that by Ding (1998), with the difference that it is applied to a urban model, Binetti and Ciani (2002) demonstrate the impact of the zoning design on a transport model of the Italian city of Bari. For their analysis, the authors define 10 different geographical divisions used in a stochastic user equilibrium traffic assignment model. Findings form the stochastic assignment put forward a significant impact of spatial aggregation on modeling outcomes. The extent of this impact far exceeds the one observed in previous studies by Bovy and Jansen (1983) or Ding (1998). As in the case of previous work, the authors do not investigate the causes of these effects. 
Bhatta and Larsen (2011) investigate the effects of ignoring intrazonal trips on the estimation of a modal choice model (MCM). The authors assume that the omission of intrazonal trips from the estimation may induce a statistical bias in MCM since this omission is not random. In this respect, their research concludes that the omission of intrazonal trips biases, indeed, the estimation of modal choice models, and thereby their results. The reason for this is that, by their very nature, intrazonal trips have shorter journey times relatively to other trips. Ignoring them amounts to removing short trips from the estimation sample. Both the estimation sample and the results of the MCM are therefore biased by this omission.

Table 4.1 summarizes the state of knowledge with regard to the impact of intrazonal trips on modeling outcomes.

The impact of spatial division on traffic assignment is clearly demonstrated in the literature. Different authors conclude that main transportation modeling outcomes depend on the spatial resolution used to produce these outcomes. Nonetheless, the demonstrated impact is unclear. Various effects, namely: the effect of spatial design and network modeling, combine with each other to produce such impact. Does the omission of intrazonal trips contribute to this impact? And if so, to what extent? These questions are still unanswered. In order to consider these research questions, this paper describes an experimental protocol that isolates the effects of intrazonal trips from other

effects caused by the definition of zones and transportation networks. To carry out this work, a stochastic aggregation method was developed and applied to the urban area of Lyon.

\subsection{Methods}

Intrazonal trips depend on the zoning design. Each zoning produces a fixed volume of these trips. Fine zonings produce little intrazonal trips compared to coarser ones, all other things being equal. In order to study the sensitivity of assignment outcomes to the omission of intrazonal trips, a variety of spatial delineations are used by implementing a zonal aggregation procedure. For each delineation, travel demand is assigned to the transport network. Various statistical measures are then constructed on the basis of assignment outcomes and analyzed in order to characterize their statistical significance (figure 4.1).

To this end, a stochastic zonal aggregation method is developed. This method produces automatically different spatial designs of a study area starting from an initial fine spatial delineation. We refer to the method as stochastic because the shape and number of zones in each produced zoning are partly random. This approach considers both spatial division and travel assignment outcomes as random variables. The goal of this paper is to statistically characterize these variables. 
Table 4.1: Summary of the current state of knowledge on the impact of intrazonal trips on transport modeling results. Only, relevant papers published before January 2018 are considered. Number between brackets are

\begin{tabular}{|c|c|c|c|}
\hline Paper & Model & Spatial designs & Findings \\
\hline $\begin{array}{l}\text { Bovy and } \\
\text { Jansen } \\
(1983)\end{array}$ & Urban & $\begin{array}{l}3 \text { spatial } \\
\text { designs }\end{array}$ & $\begin{array}{l}\text { Impact of zoning demonstrated. Both zoning and network } \\
\text { descriptions affect assignment outcomes. This impact is } \\
\text { independent of intrazonal trips. }\end{array}$ \\
\hline $\begin{array}{l}\text { Crevo } \\
(1991)\end{array}$ & Regional & $\begin{array}{l}2 \text { spatial } \\
\text { designs }\end{array}$ & $\begin{array}{l}\text { Impact of zoning not demonstrated. Shortcomings of the } \\
\text { experimental procedure. }\end{array}$ \\
\hline $\begin{array}{l}\text { Ding } \\
(1998)\end{array}$ & National & $\begin{array}{l}8 \text { spatial } \\
\text { designs }\end{array}$ & $\begin{array}{l}\text { Impact of zoning demonstrated. The author highlights the } \\
\text { potential effect of intrazonal trips. }\end{array}$ \\
\hline $\begin{array}{l}\text { Binetti and Ciani } \\
(2002)\end{array}$ & Urban & $\begin{array}{l}10 \text { spatial } \\
\text { designs }\end{array}$ & $\begin{array}{l}\text { Impact of zoning demonstrated. The results vary in a significant } \\
\text { manner depending on the zoning. }\end{array}$ \\
\hline $\begin{array}{l}\text { Chang et } \\
\text { al (2002) }\end{array}$ & Regional & $\begin{array}{l}3 \text { spatial } \\
\text { designs }\end{array}$ & Impact of zoning and network demonstrated. \\
\hline Bhatta and Larsen & Regional & - & $\begin{array}{l}\text { Impact of intrazonal trips on a modal choice model } \\
\text { demonstrated. }\end{array}$ \\
\hline$(2011)$ & & & \\
\hline $\begin{array}{l}\text { Jeon et } \\
\text { al }(2012)\end{array}$ & Urban & $\begin{array}{l}2 \text { spatial } \\
\text { designs }\end{array}$ & Impact of zoning and network demonstrated. \\
\hline $\begin{array}{l}\text { Walker } \\
(2014)\end{array}$ & Urban & $\begin{array}{l}9 \text { spatial } \\
\text { designs }\end{array}$ & $\begin{array}{l}\text { Inconclusive findings. Impact of zoning and its significance } \\
\text { not demonstrated. }\end{array}$ \\
\hline
\end{tabular}




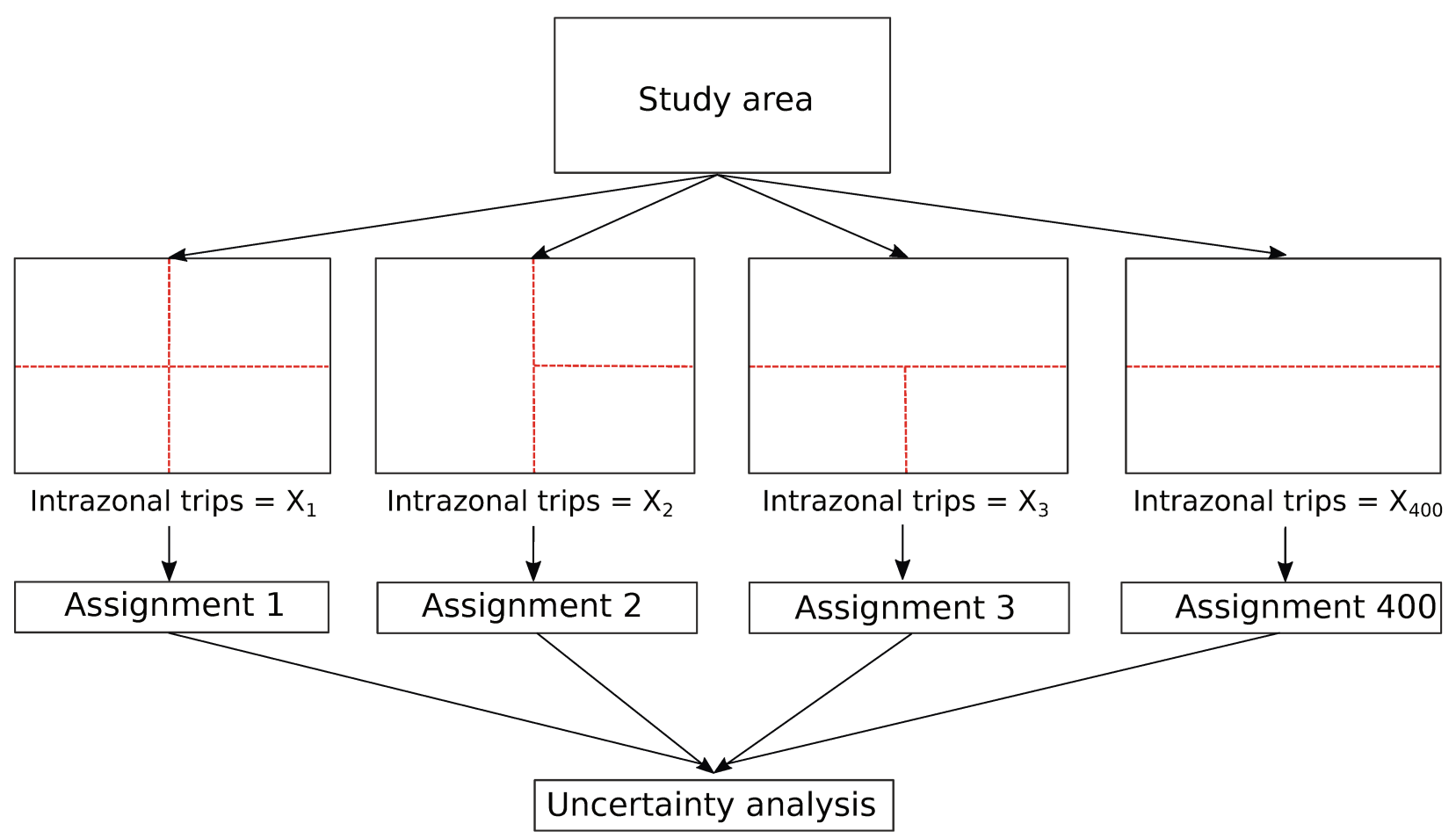

Figure 4.1: The experimental protocol. The study area is divided using different spatial designs. For each zoning an assignment procedure is computed and the variability of results is analyzed

\subsubsection{Zonal Aggregation}

The aggregation algorithm produces different spatial divisions starting from a fine initial zoning. It operates on the principle of automatically and iteratively grouping zones together to form macrozones, while complying with a number of criteria, in particular, contiguity, form regularity (nonelongated forms), exclusivity (non-overlapping zones), uniformity of zone weights, and maximization of interzonal traffic. These criteria are often put forward in the design of Traffic Analysis Zones (Baass, 1981; Bonnel, 2004; Ding, 1998, 1994; Martínez et al., 2009; Masser and Brown, 1975; Ortúzar and Willumsen, 2011; O’Neill, 1991; You et al., 1998).

The algorithm is applied to an original division consisting of $m$ zones and operates in the following manner:

1. A random or arbitrary number $n$ for the final number of zones is selected. $n$ must be less than $m$. This number may also be chosen by the user;

2. A reference zone is randomly selected from among the $m$ zones. This is denoted by $Z$. To choose $Z$, the algorithm applies a sampling law whose probability is inversely proportional 
to the number of trips that are attracted and produced by zone $Z$. This stage ensures the uniformity criterion;

3. The neighbors of $Z$ are identified. This set of zones is denoted $V_{Z}$. This stage guarantees compliance with the contiguity criterion;

4. Zones of $V_{Z}$ that may result, if aggregated, in an irregular shape or overlapping forms are automatically discarded from $V_{Z}$. This stage ensures convexity (regular form) and exclusively (no overlapping zones) of zones;

5. The zone $Z^{\prime}$ which minimizes the exchanged traffic with $Z$ is selected from among the remainder zones of $V_{Z}$. Choosing $Z^{\prime}$ maximizes the exchanged traffic in the aggregated model. Following this stage, a zone is selected to be aggregated with $Z$;

6. Zones $Z$ and $Z^{\prime}$ are aggregated and the corresponding zoning and travel demand matrix are updated. The number of zones, $i$, of the corresponding zoning is computed;

7. Set $m$ to $i$. The algorithm is rerun from stage 2 until $i=n$.

Each implementation of the algorithm results in a spatial division which is defined by an iterative aggregation procedure in which both the shape and the number of zones are randomly selected. Thus, several divisions of the same study area can be constructed. In particular, for a given final number of zones $n$, several divisions can be generated depending on random selection of intermediate zones to be merged.

The weight uniformity between zones is guaranteed by a so-called proportional random selection procedure in which the probability of selecting a reference zone is inversely proportional to its weight as represented by the sum of its trip attractions and productions. At the end of the process, the aggregated zones will likely have uniform weights. Achieving uniformity in this way means that the automatic aggregation avoids generating zones which produce or attract disproportionate amounts of traffic as this bears the risk of artificially congesting the network at the vicinity of these zones.

The criteria of convexity and exclusivity (non-overlapping zones) ensure the selection of zones with regular shapes: convex and non-elongated zones which do not contain any islands.

The aggregation procedure is automatic and needs no intervention from the modeler. Computation times depend mainly on the final number of zones $n$, the criteria to meet and whether these criteria are in contradiction with each other or not, as can sometimes occur: for example, the need to meet the criteria of shape regularity and the maximization of exchanged traffic.

The aggregation method was applied to the study area of Lyon (figure 4.2). In population terms, this area is the second largest urban area in France. Initially, it was divided up using the IRIS administrative division. The IRIS zoning is the most fine-grained administrative delineation for the dissemination of reliable socio-economic data in France (Insee, 2017). Each IRIS zone contains an 
average of 2,000 inhabitants. The urban area of Lyon contains 777 such zones. Given this detailed zoning, 400 divisions are constructed by applying the above aggregation procedure and manually selecting the final number of zones. In this paper, we retain 8 spatial resolutions, with 50, 100, 200, 300, 400, 500, 600 and 700 zones. Fifty alternatives are constructed for each level of resolution. In all, 400 different divisions are studied.

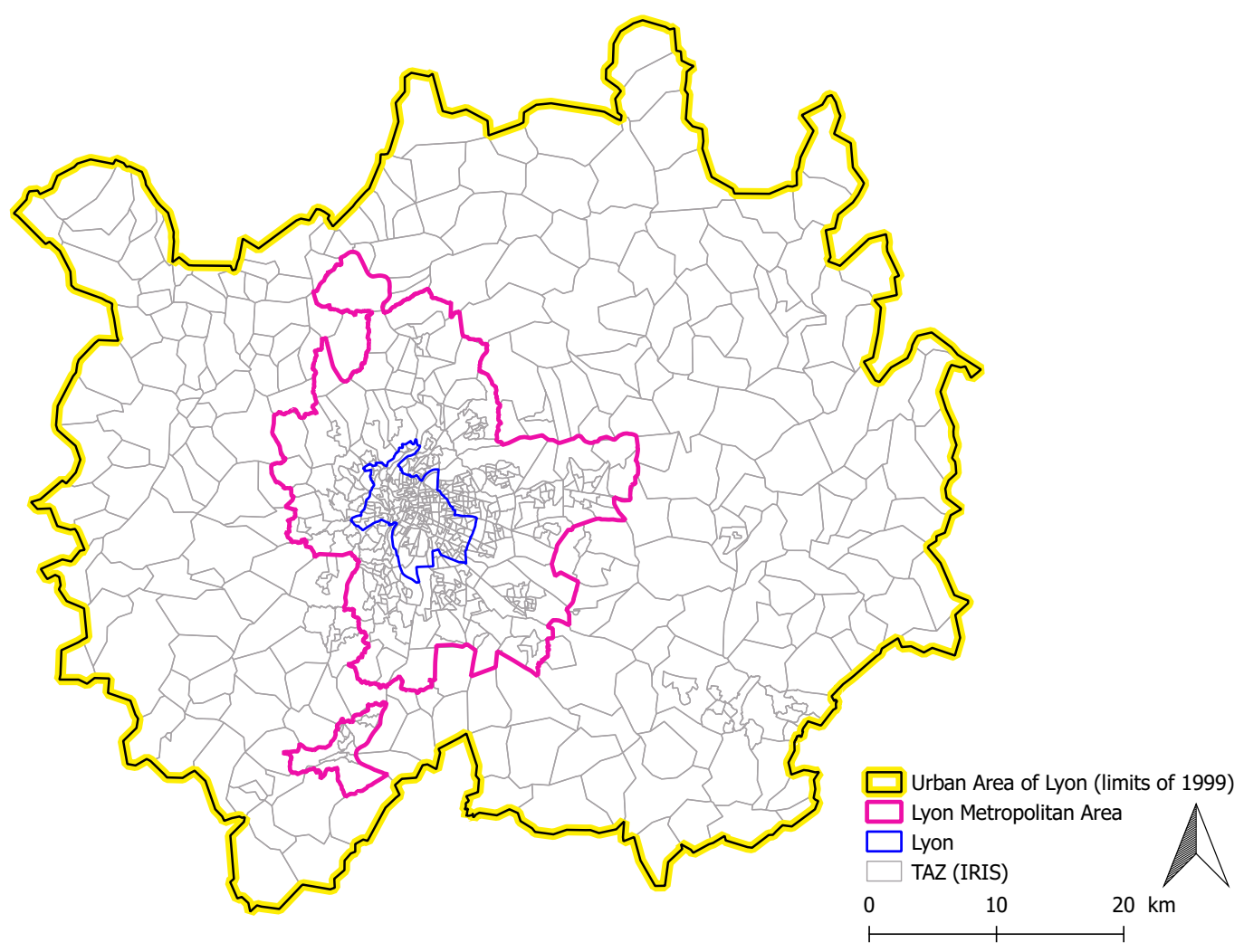

Figure 4.2: The urban area of Lyon (limits of 1999). The urban area is divided into 777 TAZ (IRIS) (data produced by Laboratoire Aménagement Économie Transports and IGN: Institut national de l'information géographique et forestière)

\subsubsection{Traffic Assignment}

For each zoning, the experimental protocol consists of assigning the corresponding travel demand to the transport network (figure 4.3). In order to isolate the effect of intrazonal trips from other aggregation effects, the definition of the network is kept constant for all aggregation levels and the definition of zone connectors is adapted for each spatial delineation. 


\subsubsection{Transport Network}

The transport network is derived from the NAVTEQ GPS dataset (figure 4.3). It is one of the most detailed network representations on the study area. The network was initially calibrated on the SIMBAD LUTI simulation platform of Lyon (Nicolas et al., 2009). The network compounds all roads, from local streets to high speed motorways. Network links are divided into 5 hierarchies given their speeds and road capacities (table 4.2). For each spatial delineation, the definition of the network is kept constant.

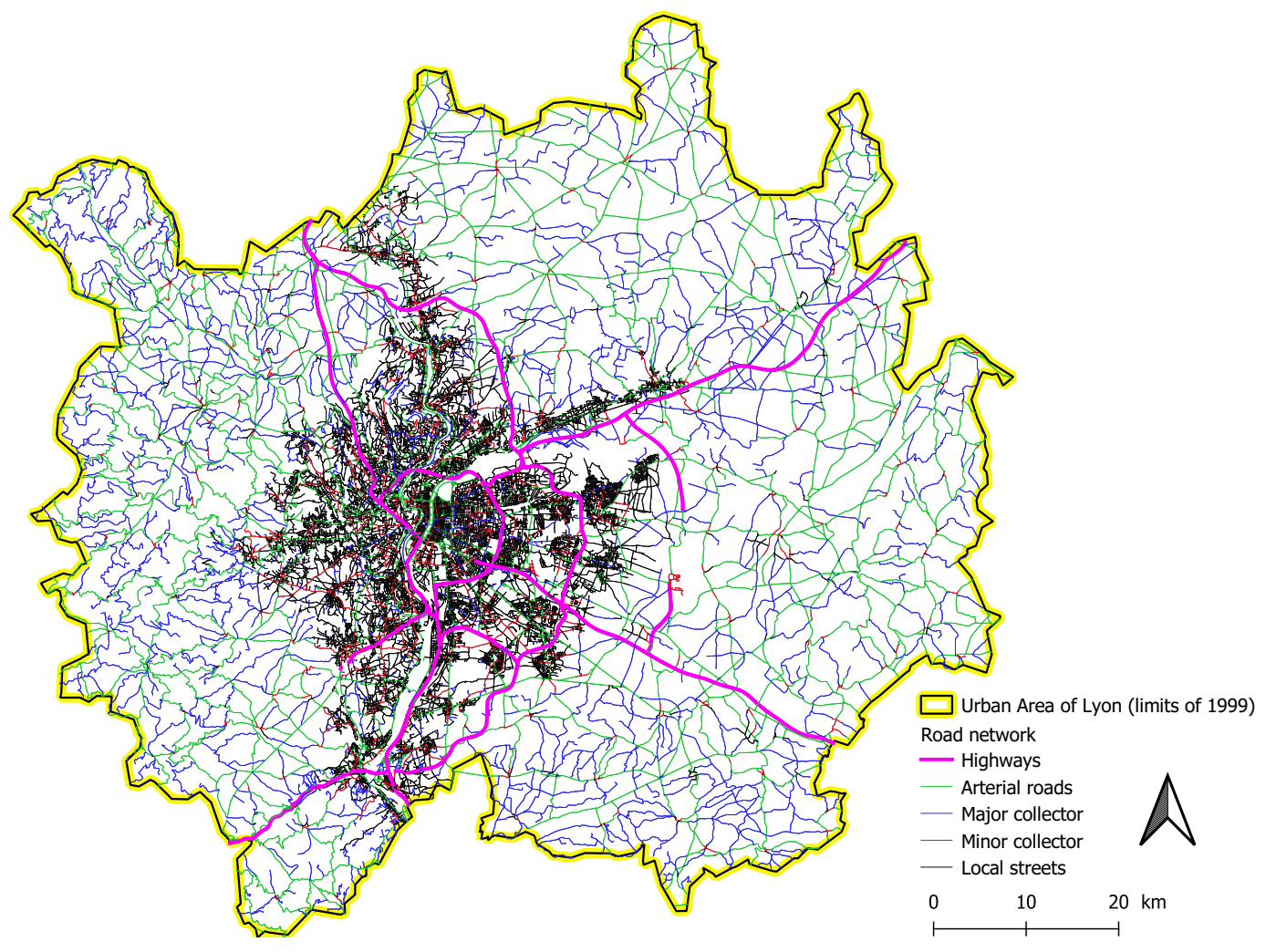

Figure 4.3: The transport network of the Urban Area of Lyon

Table 4.2: Characteristics of the network of Lyon according to road hierarchy. Mean values are computed using the arithmetic mean

\begin{tabular}{clcccc}
\hline $\begin{array}{c}\text { Network } \\
\text { hierarchy }\end{array}$ & Link category & $\begin{array}{c}\text { Number of } \\
\text { links }\end{array}$ & $\begin{array}{c}\text { Total } \\
\text { length }(\mathrm{km})\end{array}$ & $\begin{array}{c}\text { Average capacity } \\
(\text { veh } / \mathrm{h})\end{array}$ & $\begin{array}{c}\text { Average Free } \\
\text { speed }(\mathrm{km} / \mathrm{h})\end{array}$ \\
\hline 1 & $\begin{array}{l}\text { Highway } \\
\text { roads }\end{array}$ & 576 & 499 & 4,265 & 102 \\
2 & Arterial roads & 12,976 & 4,900 & 1,125 & 60
\end{tabular}




\begin{tabular}{|c|c|c|c|c|c|}
\hline $\begin{array}{l}\text { Network } \\
\text { hierarchy }\end{array}$ & Link category & $\begin{array}{l}\text { Number of } \\
\text { links }\end{array}$ & $\begin{array}{c}\text { Total } \\
\text { length }(\mathrm{km})\end{array}$ & $\begin{array}{c}\text { Average capacity } \\
(\text { veh } / \mathrm{h})\end{array}$ & $\begin{array}{l}\text { Average Free } \\
\text { speed }(\mathrm{km} / \mathrm{h})\end{array}$ \\
\hline 3 & $\begin{array}{l}\text { Major } \\
\text { collector } \\
\text { roads }\end{array}$ & 7,761 & 4,749 & 1,078 & 62 \\
\hline 4 & $\begin{array}{l}\text { Minor } \\
\text { collector } \\
\text { roads }\end{array}$ & 8,342 & 1,246 & 910 & 50 \\
\hline 5 & Local streets & 55,883 & 7,278 & 407 & 31 \\
\hline $\begin{array}{c}\text { Entire } \\
\text { network }\end{array}$ & All roads & 85,538 & 18,672 & 903 & 50 \\
\hline
\end{tabular}

\subsubsection{Zone Connectors}

In order to isolate the impact of ignoring intrazonal trips, the effect of zone connectors must be neutralized. Zone connectors are used to model access and egress to the network. They attach zone centroids to the network through connection nodes. They are usually defined on the basis of the geographical position of centroids and thus depend on the zoning design. Given our experimental protocol, connectors are redefined after each aggregation on the basis of new aggregated zones. The impact of this on journey times and other assignment indicators is straightforward (Manout et al., 2018; Sean Qian and Zhang, 2012). In order to reduce this impact, we have modified the definition of zone connectors. For each spatial division, connectors are defined according to their original state in the original zoning of 777 zones.

In practice, connectors of each macro-zone correspond to all connectors of its subzones, and the same is true for connection nodes. This means that travel demand gains access to the network using the same access times and the same connection nodes as with the original division. This minimizes the effect of zone connectors. 


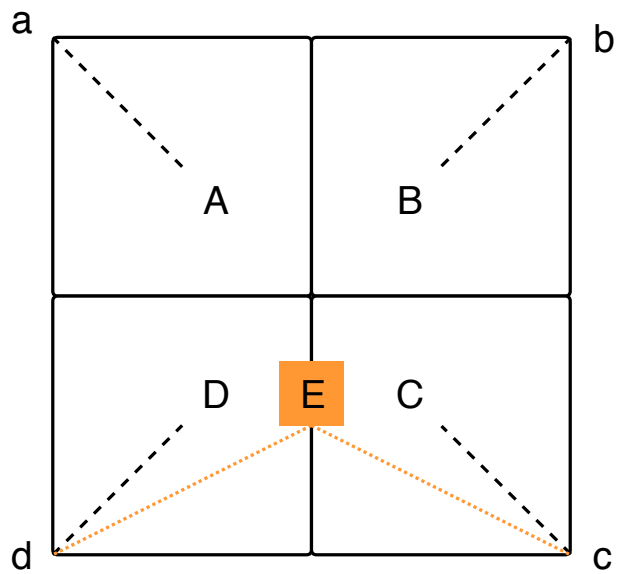

Figure 4.4: Conservation of connectors during zonal aggregation. Four zones: A, B, C, and D connected to their corresponding connection nodes: a, b, c, and d with connectors A-a, B-b, C-c, and D-d respectively. The connectors of macro-zone E are the set of the connectors of micro-zones C and D (Source: adapted from a suggestion of a reviewer of the paper)

The Aggregation of zones $\mathrm{C}$ and $\mathrm{D}$ in the example above (figure 4.4) results in a macro-zone $\mathrm{E}$ whose connectors E-c and E-d re-use the same connection nodes, c and d, and connector lengths, i.e. $\|\mathrm{E}-\mathrm{c}\|=\|\mathrm{C}-\mathrm{c}\|$ and $\|\mathrm{E}-\mathrm{d}\|=\|\mathrm{D}-\mathrm{d}\|$.

Despite the conservation principle of the definition of centroid connectors, the impact of connectors cannot be totally neutralized. For instance, when zones $\mathrm{C}$ and $\mathrm{D}$ are merged to form zone E, trips from zones $\mathrm{C}$ to $\mathrm{A}$, that used to be distributed between links $\mathrm{C}-\mathrm{c}-\mathrm{b}-\mathrm{a}-\mathrm{A}$ and $\mathrm{C}-\mathrm{c}-\mathrm{d}-\mathrm{a}-\mathrm{A}$, are now using only links $\mathrm{E}-\mathrm{d}-\mathrm{a}-\mathrm{A}$ since this is the shortest route to get to A from E. Links $\mathrm{c}-\mathrm{d}$ and c-b-a will no longer be used by these trips and a decrease in average link flows will arise. This problem is dubbed the rerouting problem. Given the experimental protocol, the rerouting problem is unavoidable, and the authors acknowledge its existence and its potential impact on modeling results. Therefore, results drawn from the current research relate to the impact of ignoring intrazonal trips from assignment but also to the rerouting problem.

For each division of the study area, a deterministic user equilibrium (DUE) traffic assignment of travel demand is performed for the evening peak hour 6-7PM. The maximum number of iterations of convergence of the DUE is set at 20 with a relative gap of $10^{-4} .400$ assignments are performed, and a variety of indicators are measured and analyzed after each assignment. These indicators are as follows: average link flows, average link speeds, congestion ratio, and total vehicle-kilometers of travel (VKT). The computation of these measures, except VKT, is weighted by the length of road links to avoid any bias arising from link segmentation.

The average link flow is computed on the entire network and on each road category. On each network link, the link flow is the average number of cars using this link in a period of time of one 
hour (in vehicles/hour). For a road category, the average flow is the weighted mean of all link flows on this category. The mean is weighed by the length of links. For the entire network, the average flow is the weighted mean of all road categories' flows. The average link speed is similar to that of link flow computed on the speed outcome.

The congestion ratio is the ratio between link flow and its nominal capacity. In transportation modeling, the capacity of a link refers to the maximum sustainable car flow expressed in vehicles/hour $(v e h / h)$ this link can convey in prevailing conditions (Transportation Research Board, National Research Council, 2000). The capacity of a link is tightly dependent on its physical characteristics: geometry, number of lanes, speed limit, crossing characteristics, etc. (table 4.2). A congestion ratio over 1 depicts a congestion situation and vice versa.

The Vehicle-Kilometers of Travel (VKT) indicator is the total mileage of all cars in the model, i.e. the sum of all traveled distances.

\subsection{Results}

Despite our efforts to isolate the impact of ignoring intrazonal trips in assignment models, subsequent results are not exclusively due to this omission but might also be dependent on the rerouting problem as stressed before.

Results show that main assignment outcomes are impacted by the omission of intrazonal trips. In particular, average link flows, network speeds and congestion ratios vary significantly with intrazonal trips. This is especially noticeable in coarse models. The extent of this impact varies also according to the category of network links.

\subsubsection{Impact on traffic}

Average link flows on the network vary significantly with the rate of intrazonal trips (figure 4.5). As one would expect, average link flows decreases as the rate of intrazonal trips increases with a high statistical correlation (-0.97). This correlation varies according to the category of the network and has an exponential shape (figure 4.5). For some road categories, an asymptotic effect, or threshold effect, is observed (sub-figure $b$ in figure 4.6). This effect is more noticeable on highway roads (category 1 in Table 4.2) than on the rest of the network.

The entire network is impacted by intrazonal trips (figure 4.5). Average link flows on the entire network varies by $63 \%$ between the most detailed model with 777 zones and the coarsest one with 50 zones (sub-figure $a$ in figure 4.6). Simulations show also that the extent of the impact increases

as one moves lower down the network hierarchy (from sub-figure $b$ to sub-figure $f$ in figures 4.5 and 4.6). The secondary network, i.e. local streets and minor collector roads, is the most impacted 

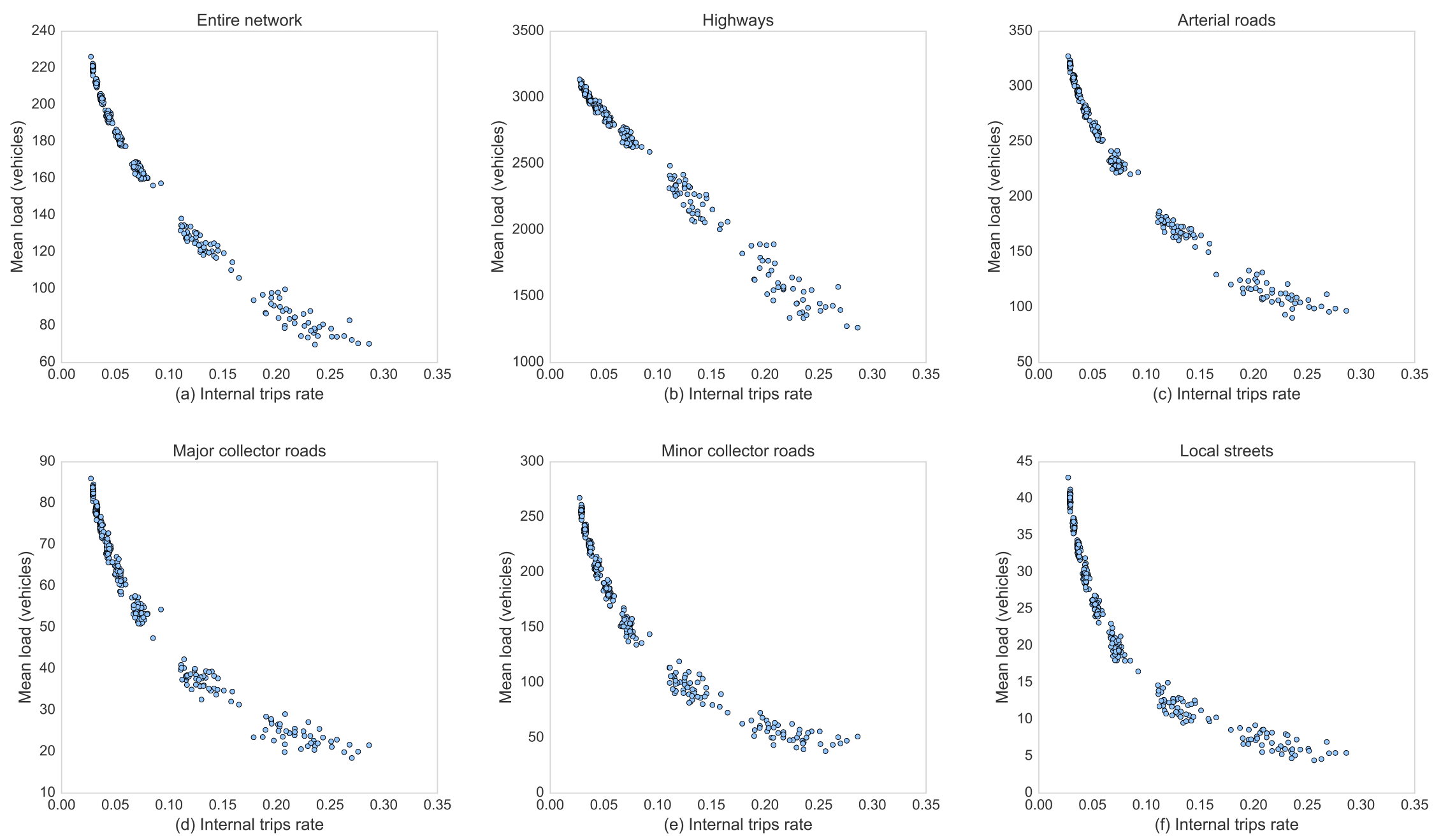

Figure 4.5: Correlation between average link flows and intrazonal trips according to road category. X-axis represents the rate of un-assigned intrazonal trips and Y-axis depicts average link flows on the network. The mean is computed using the length of links as a weight. Each point in the graphic is a traffic assignment outcome 

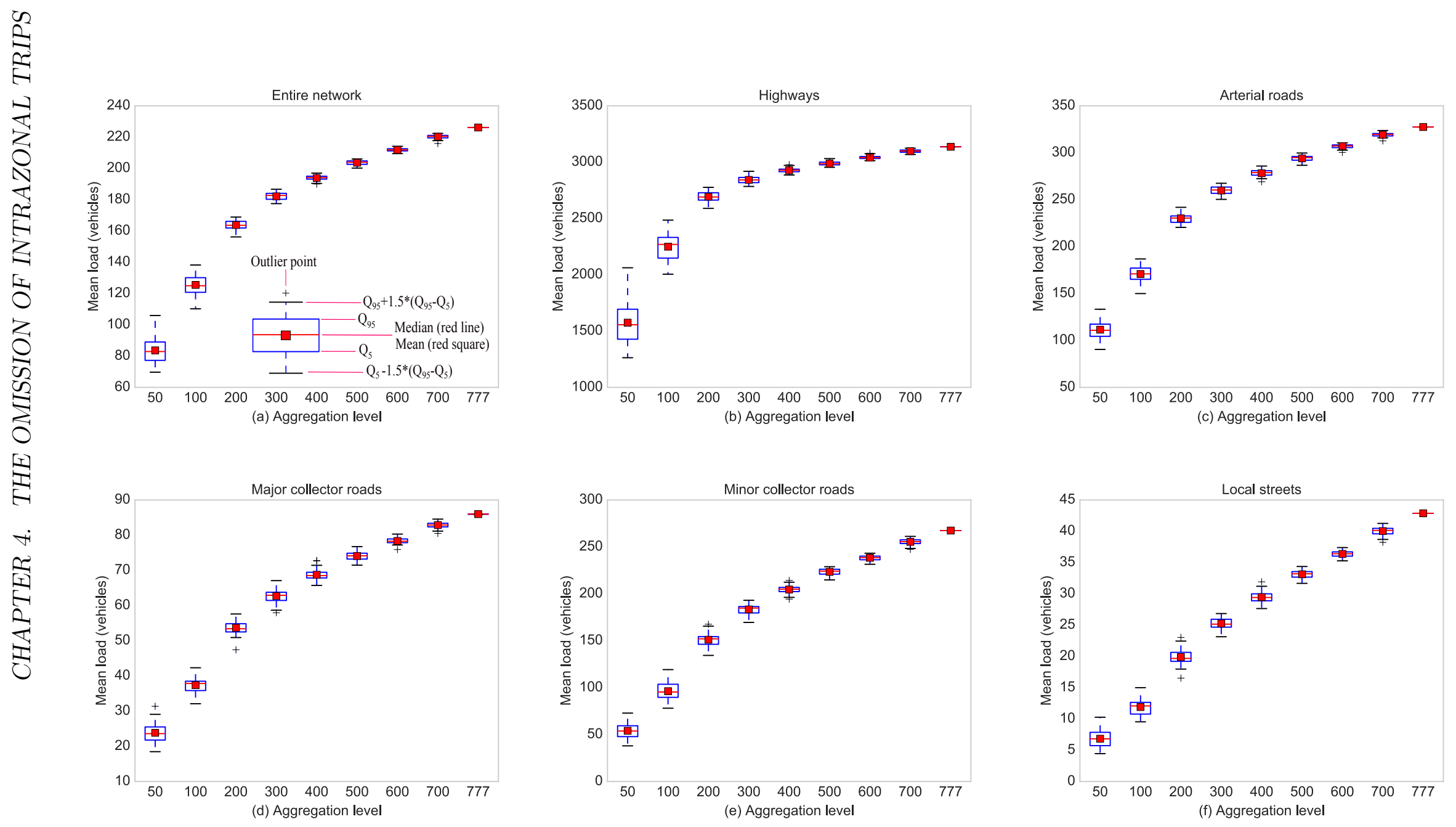

Figure 4.6: Impact of the level of zonal aggregation on average links flows according to road category. X-axis represents zonal aggregation level and Y-axis depicts average link flows. The mean is computed using the length of links as a weight. For each level of aggregation, 50 alternatives are tested 
network by the omission of intrazonal trips. Average link flows on these roads varies by almost $80 \%$ (sub-figures $e$ and $f$ in figure 4.6). For comparison purposes, this variation is of $50 \%$ on the primary network, i.e. highway and arterial roads.

The distribution of average link flows on the entire network has a coefficient of variation between $0.5 \%$ and $11 \%$ (figure 4.6 ). This variation is due to the distribution of intrazonal trips produced by the 50 alternative spatial designs constructed for each spatial resolution. The distribution of average link flows varies also according to the category of the network and the size of zones. It increases gradually with the level of zonal aggregation.

The confidence intervals of average links flows, computed at a $5 \%$ significance level, do not overlap (figure 4.6). The observed difference between the means of the different aggregation levels is therefore statistically significant. To confirm this hypothesis, we conducted a Welch's t-test (M. B. Brown and Forsythe, 1974b). Unlike standard tests: Student's t test or ANOVA, this statistic tests the null hypothesis of equal means in populations which do not have the same variance, as is the case here. This test rejects the null hypothesis of the equality of the means (pvalue $\leq 0.000$ ) and confirms that each aggregation level has a statistically different average link flow. Thereby, the omission of intrazonal trips has a significant statistical impact on network flows.

\subsubsection{Impact on journey speeds}

Like average link flows, average link speeds are also affected by the omission of intrazonal trips (figures 4.7 and 4.8). An increase in the proportion of these short trips leads to an increase in the average link speeds with a positive correlation of 0.91 . However, this impact is less marked than with average flows. The average speed on the entire network increases by almost $6 \%$ as one moves from the finest model with 777 zones to the coarsest one with 50 zones (sub-figure $a$ in figure 4.8). In the case of the detailed model with 777 zones, the majority of network links are not congested. Only $3 \%$ of the entire network has a congestion ratio of over 1. Traffic flows freely on almost all the network, and particularly on the secondary one. Therefore, the omission of intrazonal trips increases slightly the speed since the level of service of the network is already good. This correlation has also an exponential shape (figure 4.7); nevertheless, the asymptotic effect observed in average link flows is less pronounced in the case of average speeds (sub-figure $b$ in figure 4.8).

The omission of intrazonal trips impacts average link speeds on the overall network, and the extent of this impact differs between road categories (figure 4.7). This effect is particularly noticeable on highway roads where average speed increases by almost $16 \%$ (sub-figure $b$ in figure 4.8); whereas the average increase on the entire network is of $6 \%$ (sub-figure $a$ in figure 4.8). The reason for this is that highway roads are relatively the most congested links in the transportation network of Lyon at the evening peak-hour. Almost $28 \%$ of highway roads have a congestion ratio of over 1 . In coarse models, the omission of intrazonal trips leads to a rapid and a fictitious increase in average speed on this road category. This impact is statistically proved by the non-overlapping $5 \%$ confidence 

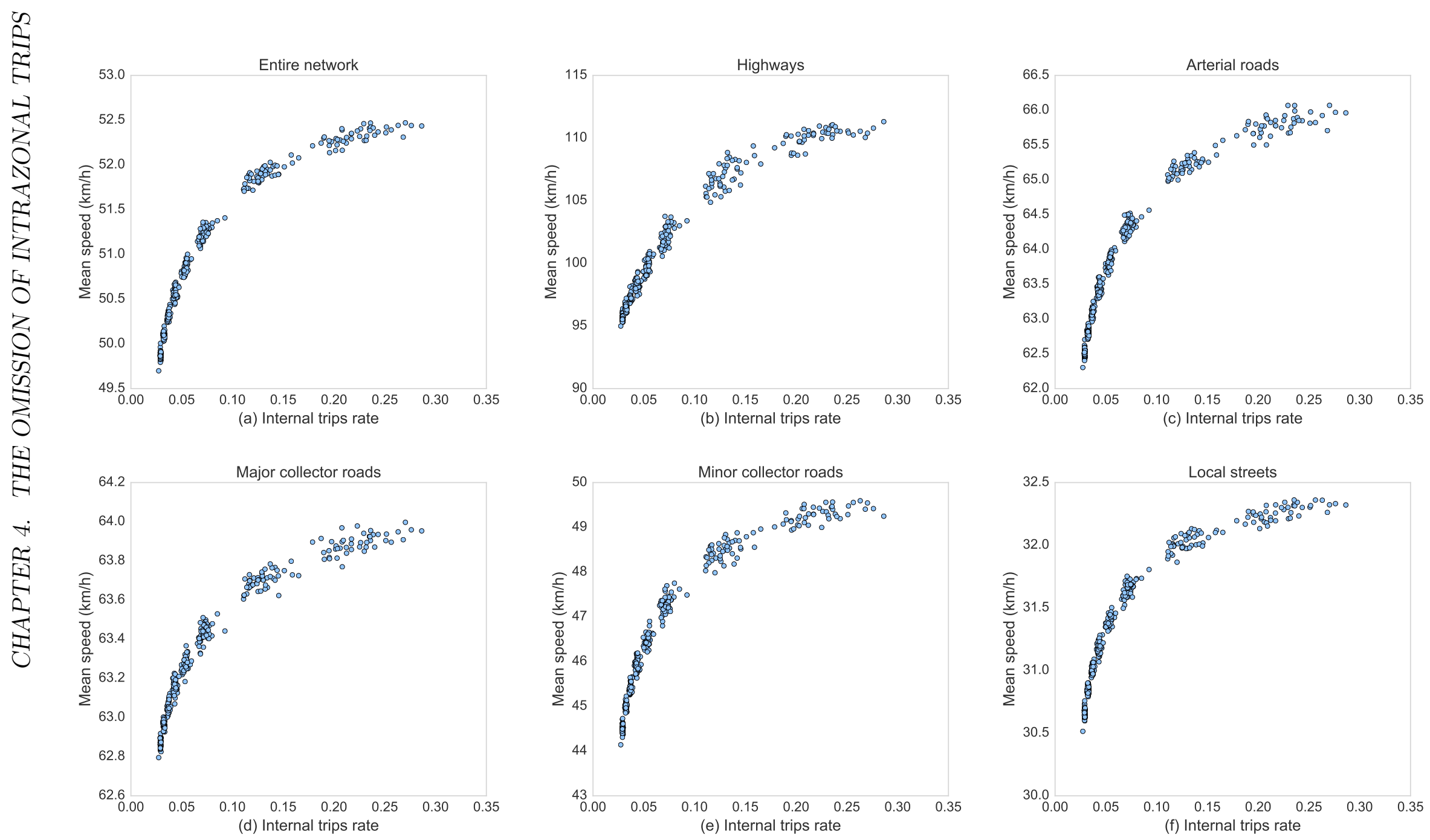

Figure 4.7: Correlation between average link speeds and intrazonal trips according to road category. X-axis represents the rate of the un-assigned intrazonal trips and Y-axis depicts average link speed. The mean is computed using the length of links as a weight. Each point in the graphic is a traffic assignment outcome 

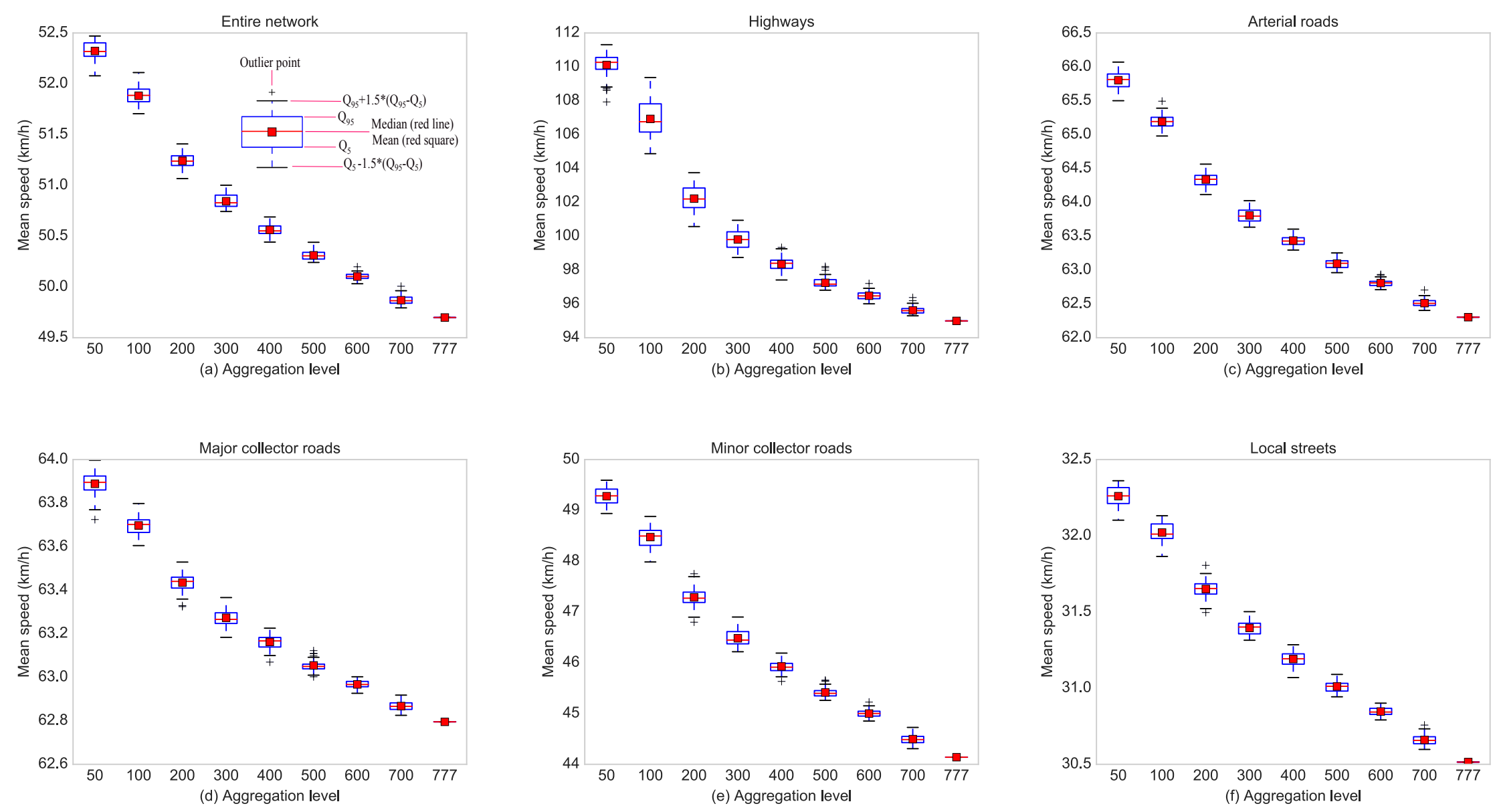

Figure 4.8: Impact of level of zonal aggregation on average link speeds according to road category. X-axis represents zonal aggregation level and Y-axis depicts average link speed. The mean is computed using the length of links as a weight. For each level of aggregation, 50 alternatives are tested 
intervals and by the Welch's t-test (pvalue $\leq 0.000$ ) (figure 4.8).

For a given number of zones (50 for example), the spread of the distribution of average speeds is low: the highest coefficient of variation does not exceed $0.12 \%$. As in the case of link flows, the standard deviation of average speeds increases with the volume of intrazonal trips and differs between road categories and spatial designs.

\subsubsection{Impact on congestion ratio and total vehicle-kilometers of travel}

The omission of intrazonal trips in traffic assignment is also detrimental to the congestion ratio and to the total mileage (in vehicle-kilometers). These assignment outcomes are highly correlated with intrazonal trips with an average correlation of -0.94 . When the magnitude of intrazonal trips increases, i.e. when the number of zones decreases, the congestion ratio (figure 4.9) and VKT fall (figure 4.10).

\subsection{Discussion}

The omission of intrazonal trips has a significant impact on main traffic assignment outcomes. These outcomes are highly correlated with the rate of omitted intrazonal trips. Some of them also exhibit an asymptotic effect when the division exceeds a certain number of zones.

\subsubsection{Correlation}

Ignoring intrazonal trips in assignment models biases main traffic assignment outcomes. As zonal aggregation proceeds, the volume of intrazonal trips increases. Their relative increase takes place at the expense of interzonal traffic and reduces the volume of assigned car trips. As a consequence, congestion levels, travel times, and total vehicle kilometers are underestimated, and average speed overestimated. This effect increases as the spatial design becomes coarser. Therefore, ignoring intrazonal trips in the assignment models generates a fictitious free-flow situation where congestion levels are underestimated and accessibility scores overestimated. This can undermine modeling accuracy and reliability, and mislead policy decision.

Ignoring intrazonal trips when conducting traffic assignment produces a fictitious free-flow situation in the model. This intuitive finding contrasts partially with findings from Ding (1998) and Binetti and Ciani (2002). Their research into the impact of zoning on assignment shows an opposite tendency: the level of saturation, i.e. the congestion ratio increases with intrazonal trips. The authors in question do not explain the causes of this unexpected positive correlation. However, 


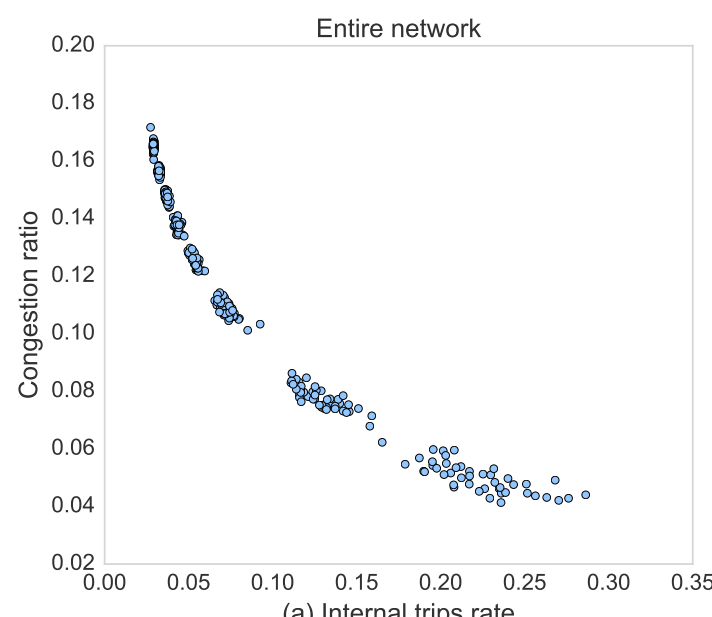

(a) Internal trips rate

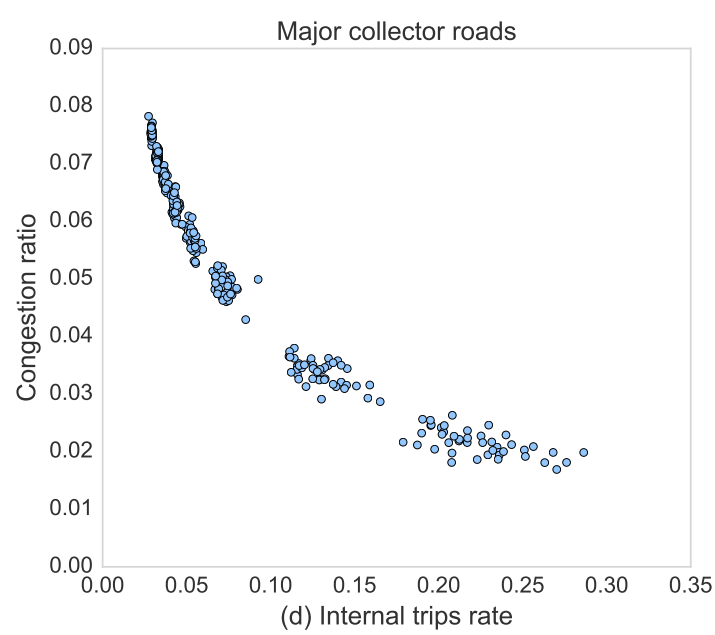

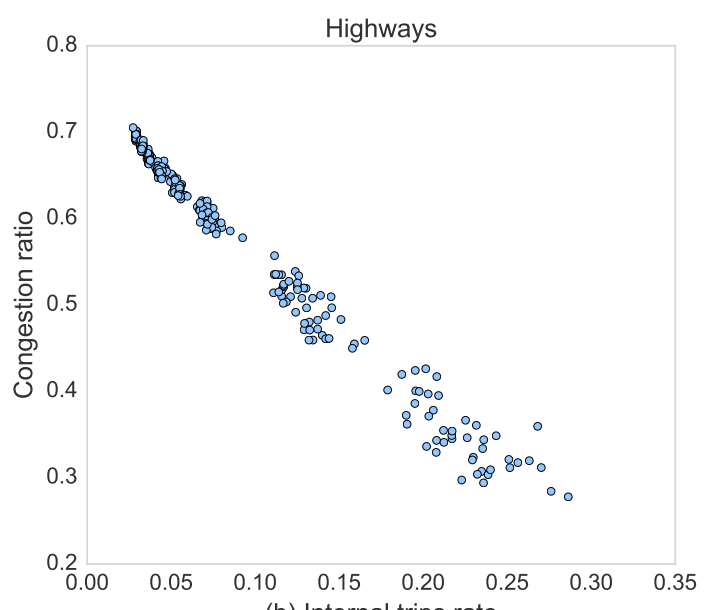

(b) Internal trips rate

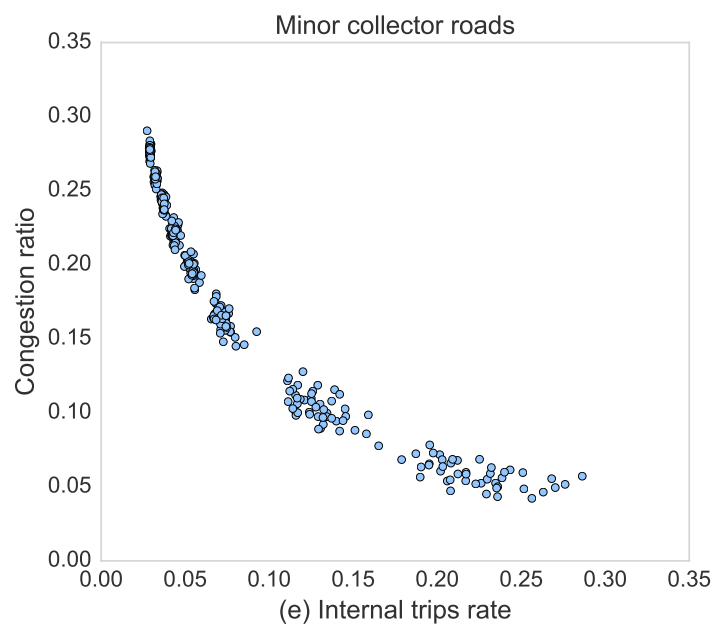

Figure 4.9: Correlation between congestion ratio and intrazonal trips according to road category. X axis represents the rate of the un-assigned intrazonal trips and $\mathrm{Y}$ axis depicts congestion ratio. The mean is computed using the length of links as a weight. Each point in the graphic is a traffic assignment outcome
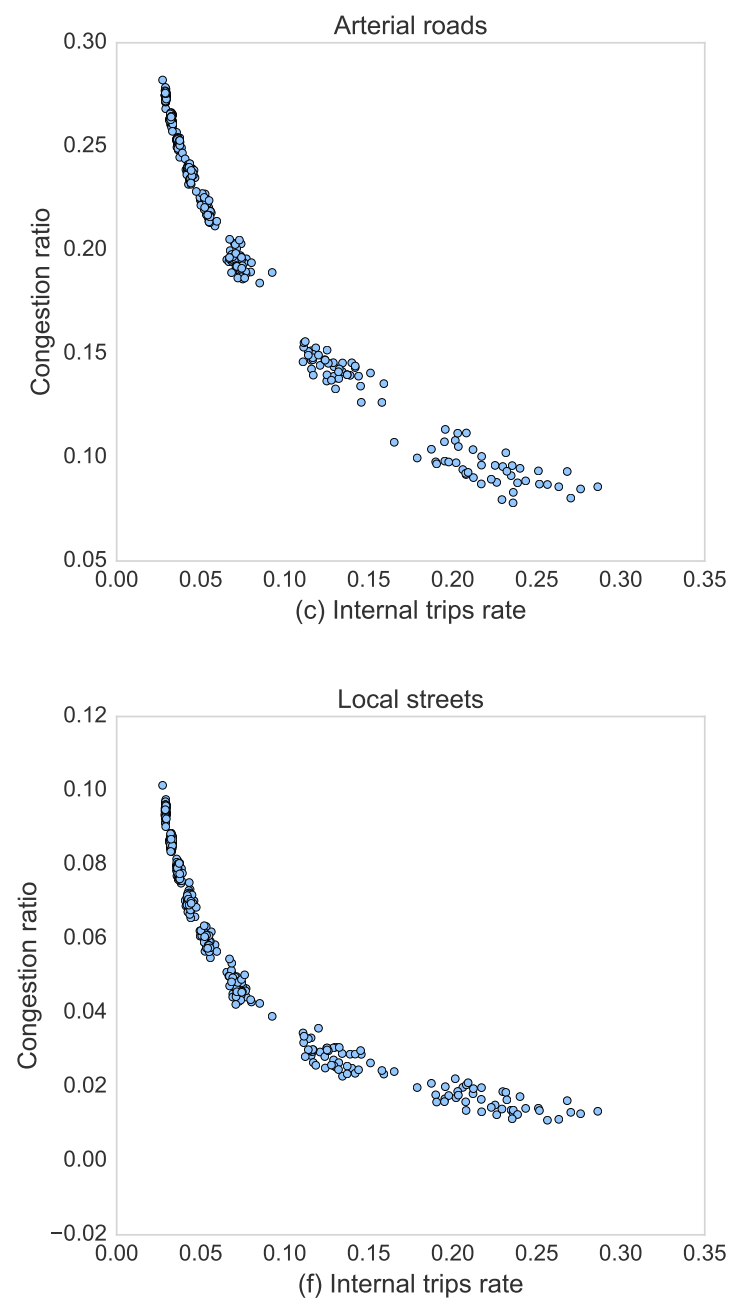

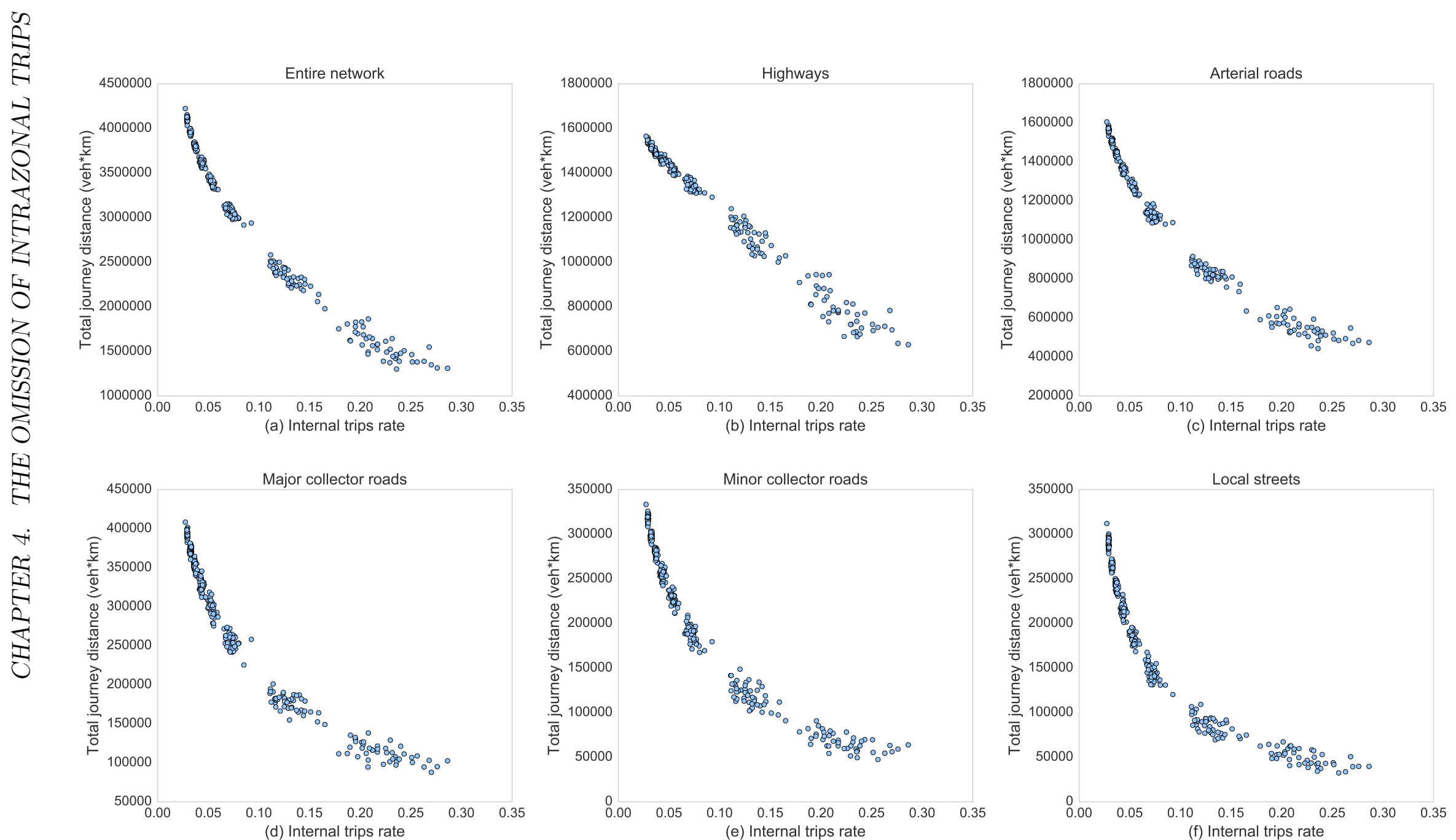

Figure 4.10: Correlation between total vehicle kilometers of travel (VKT) and intrazonal trips according to road category. X axis represents the rate of the un-assigned intrazonal trips and $\mathrm{Y}$ axis depicts total VKT. Each point in the graphic is a traffic assignment outcome 
this finding may be explained by the experimental protocol used in both research works. In their research, the authors do not isolate the effects of intrazonal trips from other spatial aggregation effects, in particular, from the impact of zone connectors. Indeed, as zones are aggregated, trip productions and attractions increase as well, while the average number of connectors remains stable. Distributing aggregated travel demand between a limited numbers of connection nodes may result in a local congestion in the vicinity of connection nodes. This situation induces an increase in saturation levels around these nodes. This phenomenon is known as the fictitious congestion due to connectors (Bovy and Jansen, 1983; Mann, 2002). The fictitious free-flow situation generated by the omission of intrazonal trips is countered by the fictitious congestion caused by zone connectors. This is probably why previous research on the subject did not outline the specific and significant impact of ignoring intrazonal trips and this is why our paper set out to study the specific effect of intrazonal trips independently of other effects. For this same reason, the definition of centroid connectors is adapted.

\subsubsection{Asymptotic effect or threshold effect}

The existence of an asymptotic effect is consistent with the observations of Bovy and Jansen (1983) and Ding (1998) who also noted that several assignment outcomes converge above a certain number of zones. However, findings from our research show that the existence and significance of this effect varies from one indicator to another and, above all, according to the road category. Only average link flows, congestion ratio, and total vehicle-kilometers of travel exhibit a noticeable convergence that is limited to the primary network. In the case study of Lyon, this convergence occurs between 300 and 500 zones where the share of omitted intrazonal trips does not exceed $5 \%$ of total trips (figure 4.11). Local streets and the secondary network in general, are not concerned with this effect. Assignment outcomes on the secondary network vary significantly whatever the number of zones is.

It is important to stress that the threshold effect is not an artefact of setting bounded values on studied indicators. In fact, network links have finite speed and capacity limits. These indicators are limited by the physical nature of the infrastructure (speed limit, number of lanes, etc.). The threshold effect under study is not directly linked to this limitation.

The asymptotic effect is due to intrazonal trips which are a direct result of the discretization of continuous space. When the study area is finely divided, the volume of intrazonal trips falls. This decrease follows an exponential shape which decreases less rapidly above 400 zones (figure 4.11). The threshold effect is a direct consequence of this finding. In the case study of Lyon, assignment outcomes begin to show signs of convergence at the 400-zone threshold. In general, the value of this threshold is around the number of zones beyond which the volume of intrazonal trips becomes stable. The stability of the share of these trips leads to a stability of assigned interzonal trips, and therefore of assignment outcomes. 


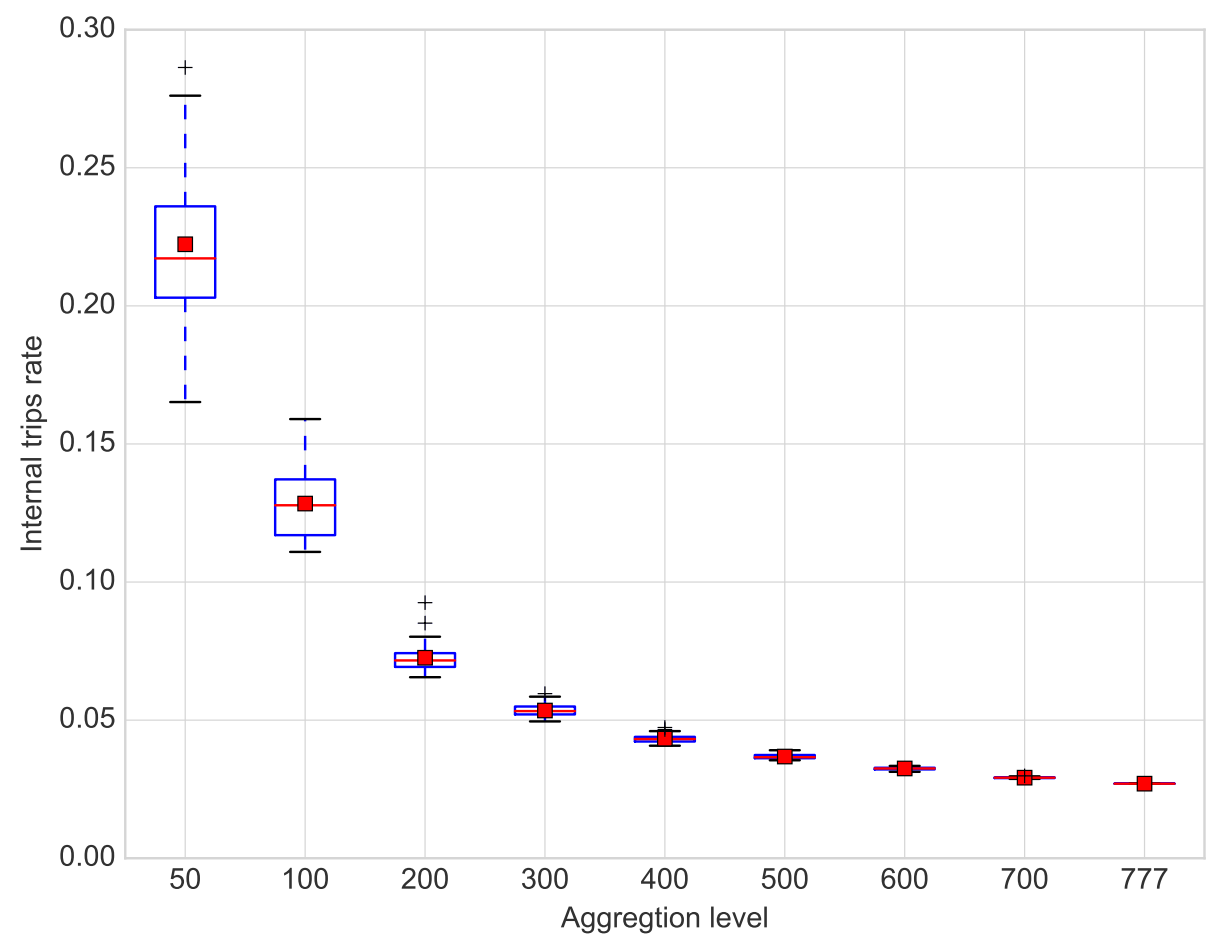

Figure 4.11: Intrazonal trips and zonal aggregation levels. For each level of aggregation, 50 alternative spatial designs are constructed

One practical consequence of the existence of the threshold effect is the convergence of modeling outcomes. For strategic models where the emphasis is on macro-effects and the primary network, there is a minimum number of zones beyond which main assignment outcomes, such as average link flows or total vehicle-kilometers converge. The marginal contribution of further refining the spatial division beyond this threshold is likely to be minimal compared to the corresponding costs of data collection, and the burden of calibration and computation. Once convergence has been achieved, it is not necessary to use more zones.

This practical conclusion may be of assistance to a modeler when making a trade-off between the cost of developing a model and its accuracy (Ortúzar and Willumsen, 2011). The existence of the threshold effect would make it possible to build aggregate zoning systems for transport models that produce accurate results at lower costs. In order to establish this threshold, it is not necessary to reapply the overall method described here (stochastic zonal aggregation followed by a batch of traffic assignments). It is sufficient to determine the threshold at which the volume of demand begins to stabilize as the number of zones is increased. In the case of Lyon, convergence emerges around an intrazonal rate of $5 \%$ or 400 zones. 


\subsubsection{Impact on the secondary network}

Findings suggest that the level of service on the secondary network is more affected by the omission of intrazonal trips than that on the primary network. This is because zonal aggregation merges contiguous zones and transforms the interzonal travel demand between them into intrazonal trips (figure 4.4). This interzonal traffic mainly uses the secondary network connecting the two aggregated zones. As aggregation proceeds, the secondary network loses its principal users which are considered, after aggregation, as intrazonal demand and ignored by the assignment model. This observation also explains the relative stability of the results of the primary network which, up to a certain level of aggregation, is mainly used for long distance trips between non-adjacent zones which cannot be merged by the aggregation procedure.

Road volumes on the secondary network are highly dependent on intrazonal trips. Since this network often operates under uncongested conditions, this dependency is not always reflected by its level of service (speeds are less impacted than flows in this case, see figure 4.7). Nonetheless, it is essential to ensure consistency between the resolution of the network and the zoning. Our results stress that it is not a valid practice to analyze the lowest road hierarchy especially in coarse models. Any analysis would be biased by the fictitious free-flow situation induced by the omission of intrazonal trips. It is important to ensure consistency between the levels of detail of the network and the spatial resolution when building a transportation model or, at least, when analyzing its outcomes. However, network and zoning data are often provided by different data providers. Zoning frequently follows administrative divisions used in data collection. Network data are provided by a variety of sources, which are frequently not administrative, such as NAVTEQ or other network data providers. Consequently, transport models frequently make use of different representations of space and network.

\subsubsection{Size and shape effects}

Computer simulations show that assignment outcomes vary not only according to the number of zones, but also with their shape (figures 4.6 and 4.8). For each level of zonal aggregation, 50 variant divisions were constructed. For a same number of zones, assignment outcomes vary according to these variants. This finding reminds one of the effects of size and shape of the MAUP (Modifiable Areal Unit Problem) described by Openshaw (1977a), (1983). In the case of traffic assignment, the impact of size seems to outweigh that of shape. Taking the network as a whole, the standard deviation of average link flows due to shape ranges between 1 and 9 vehicles/hour, while that for size effect far exceeds this level (sub-figure $a$ in figure 4.6).

Finally, the shape effect introduces a variation that decreases with the number of zones. The standard deviation of the aforementioned outcomes increases as aggregation proceeds. This is due to the stochastic aggregation method, in which the possible event space increases with the number of 
zones to be aggregated. Aggregation starting from the finest model of 777-zone introduces variability as zones are aggregated. In this regard, the algorithm has more alternatives for reaching a 50-zone division than it does with a 700-zone division, that is why variance of outcomes increases when the final number of zones decreases.

\subsection{Conclusion}

The aim of this research was to investigate the impact of ignoring intrazonal trips in assignment models and to statistically characterize this impact. By its very nature, traffic assignment ignores intrazonal trips. The precise impact of this omission has not been addressed specifically in the literature, although it has frequently been discussed as a side issue to other questions such as zoning. To tackle this problem, we have attempted to study the impact of intrazonal trips independently of other effects by applying an uncertainty analysis framework.

As in the case of modal choice models (Bhatta and Larsen, 2011), ignoring intrazonal trips in traffic assignment biases modeling results. The extent of the impact varies according to road hierarchy. Local streets, and the secondary network in general, are significantly biased by the omission of these trips while the primary network is unaffected by it up to a certain aggregation level. Nevertheless, whatever road category, assignment outcomes are highly correlated with the volume of intrazonal trips. Even if these results are drawn from a unique case study, one might expect similar patterns in different urban contexts. These findings raise doubts about the reliability of transport modeling results. Errors introduced by such omission contribute to the general problem of modeling errors. Therefore, it is noteworthy to assess the impact of ignoring intrazonal trips in assignment models. For this end, the reader can apply the current sensitivity analysis to any case study wherever a traffic assignment model is available. Findings from such an analysis can be helpful in choosing the right spatial resolution for a model given its objectives. These findings can also improve the reliability of modeling results by assessing their sensitivity towards the omission of intrazonal trips.

In order to minimize the impact of omission, it is possible to use a fine spatial division which minimizes the volume of intrazonal trips and, thereby, their impact. However, the fineness of zoning is frequently limited by data availability, development costs, and the goals of the model itself. This is because fine grained spatial representations may be in contradiction with the goals of the model, as is the case with strategic models which are frequently macroscopic. With such models, the existence of the convergence threshold makes it possible to select a minimum number of zones which provides accurate results while minimizing development costs and the impact of ignoring intrazonal trips.

Another solution to this problem is to ensure consistency between spatial and network descriptions during model construction. Any division generates intrazonal trips which consume some of the capacity of the network. This consumed capacity should be deducted from the capacity of the modeled network in order to avoid fictitious free-flow conditions. Thus, the description of the 
network should take account of the implicit existence of intrazonal trips associated with each level of spatial zoning. This solution requires further research in order to define a measure of consistency between the zoning, the network, and the means by which intrazonal trips are to be considered in the definition of the transport network.

Finally, findings drawn from this research are partly dependent on the rerouting problem. Given the experimental protocol, it was not possible to perfectly isolate the problem of intrazonal omission from the problem of rerouting due to the definition of centroid connectors. The current research characterizes the combined effects of these problems and it is of interest to disentangle these two effects in order to assess their specific errors and extent. For this end, further research is required.

\section{Acknowledgments}

The authors are grateful to the three anonymous reviewers of this paper. Thanks to their comments and suggestions, the current paper has been enriched and improved.

This research has been carried out thanks to the support of ForCity and PhD. Hakim Ouaras.

An earlier version of this paper has been published in Transportation journal under DOI: 10.1007/s11116-018-9951-y. 


\section{Chapter 5}

\section{On the spatial aggregation problem in traffic assignment models}

\subsection{Context}

During the last 50 years, academic research has developed a plethora of transport modeling approaches (Bonnel, 2004; Ortúzar and Willumsen, 2011). From simplistic transport models of the 60s to current individual-centric modeling frameworks, research is pushing forward modeling sophistication and complexity. Despite this research agenda, transport models are still prone to bias. In several instances, these errors are seriously detrimental to the accuracy of transport models and thus to policy instruction. Ignoring these errors or neglecting their impacts may induce serious modeling errors, mislead policy decision, and ultimately endorse inefficient urban planning schemes with unpredictable economic and social costs (Dupuy, 1975; Flyvbjerg et al., 2005; Skamris and Flyvbjerg, 1997). The spatial aggregation problem is one of these enduring yet disregarded modeling issues (Manout and Bonnel, 2018; Manout et al., 2018; Ortúzar and Willumsen, 2011). The spatial aggregation problem is induced by the common practice of modeling continuous space as discrete. The majority of standard transport models including some of the most sophisticated ones rely, by design, on this aggregate description. In assignment modeling, this practice has two major consequences: the omission of intrazonal trips and the use of centroid connectors.

Centroid connectors are artificial network links introduced to attach zone centroids to transportation network. The definition of these links and their implications in transit assignment have already been discussed and addressed in chapters $\mathbf{2}$ and $\mathbf{3}$. Intrazonal trips are trips that start and end at the same zone. These trips are often disregarded from analysis especially in traffic assignment. Only trips exchanged between zones are considered in conventional assignment models. Intrazonal trips are not captured and the effect of this omission is not acknowledged nor evaluated; yet, this deliberate omission may be a serious source of errors (Manout and Bonnel, 2018). In chapter 4, 
a statistical description of the impact of ignoring intrazonal trips in traditional traffic assignment models has been undertaken. Findings from that chapter have brought to light the shortcomings of the standard assignment in addressing the omission of intrazonal trips and the extent of the bias induced by such an omission. The current chapter builds on previous findings to suggest new assignment models that address the omission bias of intrazonal trips and by doing so the aggregation bias in assignment models.

To address this problem, transportation research and manuals suggest some solutions like using detailed zonings or substituting microsimulation models for aggregate ones. Indeed, using fine-grained spatial units can minimize the loss of intrazonal trips and therefore their impact. Nevertheless, this approach avoids tackling the problem of ignoring intrazonal trips and addresses it indirectly by minimizing its magnitude. Moreover, when refining the spatial design one should also refine the description of the network in order to ensure a minimum consistency between zonal and network descriptions (Bovy and Jansen, 1983; Chang et al., 2002; Jeon et al., 2012). In this regard, spatial refinement comes at the price of increased costs of data collection, longer computational times, and tedious calibration efforts. Furthermore, if this method can alleviate the impact of ignoring intrazonal trips, the use of detailed spatial designs is not always possible nor desirable. In practice, data privacy issues are often a barrier to the use of micro-data. In other cases, modeling resolution is deliberately chosen to be coarse as in the case of strategic and regional transport models.

Regarding the above arguments, one might legitimately argue that microsimulation models are a solution to the so-called aggregation problem. Indeed, from a theoretical standpoint, microsimulation models offer an attractive solution to the aggregation problem by reducing the modeling resolution of behavioral and spatial units. By doing so, the aggregation bias is reduced. However, if many microsimulation models are currently operational, these models are yet to become practical ${ }^{1}$. One obstinate barrier to this is micro-data availability and reliability, especially for predictivemodeling purposes. The use of synthetic data to substitute for micro-data is a common practice in microsimulation. Thus, there remains the question: To what extent microsimulation models using synthetic data are more accurate than standard models relying on aggregation, especially in predictive situations? The answer to this question is not straightforward and it is out of the scope of this dissertation (Ay et al., 2017). The current PhD research does, however, take part of a general effort to answer the above question by investigating the bias induced by using aggregate data in standard traffic assignment models and by suggesting new techniques to overcome the corresponding aggregation errors.

In the next section, a brief literature review of solutions to the omission problem in traffic assignment models is undertaken. These solutions can be of different natures and have varying degrees of success. Study objectives are stated afterwards. Four different traffic assignment strategies and 6

\footnotetext{
${ }^{1}$ Practicality is defined in chapter $\mathbf{1}$ as the ease with which a model can be applied in a useful way to answer predefined questions pertaining to the scope and objectives of the model. For a model to be practical, it needs to be: Useful, Replicable, Affordable, and Responsive.
} 
approaches are introduced in section 5.4: Methods. These methods are designed to reduce the spatial aggregation bias by assigning intrazonal trips or by reducing the impact of their omission. In section 5.5: Case studies, the contribution of these strategies is assessed in three case studies: a simple case study of 4 zones and 8 links and two more complex case studies from Sioux Falls in the US and Lyon in France. Section 5.6: Results explores in detail the contribution of each assignment strategy. In this regard, various goodness-of-fit indicators are used and compared. Finally, findings of this research are discussed and some recommendations and future work suggestions are provided in section 5.7: Discussion.

\subsection{Literature review}

Scarce research studies have addressed the problem of assigning intrazonal trips or that of appraising the impact of their omission (Bureau of Public Roads, 1964; Daganzo, 1980b, 1980a; DeCorla-Souza and Grubb, 1991; Eash et al., 1988; Horowitz, 2001; Mann, 2002; Moeckel and Donnelly, 2009; Texas Transportation Institute and State Department of Highways and public transportation, 1988). This research question has often been left on the margins of other issues like spatial design or network representation. The only existing literature on the subject investigates the question of intrazonal trips with varying degrees of success and the only papers dealing with this issue can roughly fall into three categories:

- Demand-side methods

- Supply-side methods

- Mix methods

\subsubsection{Demand-side methods}

Demand-side methods view the problem of ignoring intrazonal trips in traffic assignment as a zoning/demand problem (Daganzo, 1980b, 1980a; Horowitz, 2001; Mann, 2002). Since, intrazonal trips start and end at the same zone, demand-side methods subdivide zones into different subzones and assign intrazonal trips between these subzones. Different subdivision methods and intrazonal distributions are possible. Three different strategies are often used: sub-centroids, link subdivision, and node subdivision.

In a series of two papers, Daganzo (1980a), (1980b), proposes to overcome the aggregation bias in traffic assignment models by refining the description of space and by reformulating the traffic assignment problem. In a first paper entitled "An equilibrium algorithm for the spatial aggregation problem of traffic assignment" (Daganzo (1980b)), the author reformulates the minimization problem of the Deterministic User Equilibrium (DUE) ${ }^{2}$ as a two stage problem in order to allow the DUE to

\footnotetext{
${ }^{2}$ This approach has been also adapted to the Stochastic User Equilibrium (SUE) by Leurent et al. (2011).
} 
manage zones with multiple centroids, i.e. sub-centroids. This strategy relies on the subdivision of zones with a special emphasis on minimizing computational costs. Different assumptions are made in line with this purpose like setting a constant travel time on centroid connectors. Since the work of Beckmann et al. (1956), the traffic assignment problem has been only carried out for zones with only one centroid. That is because the use of sub-centroids introduces a computational burden that the new formulation of Daganzo controls for. Still, only few sub-centroids can be used to keep the problem tractable given computation capacities of the 80s. In a second paper, Daganzo (1980a) extends the capacity of traffic assignment models to allow for an infinite number of sub-centroids and thus to propose a continuous description of space, i.e. population/activity distributions using off-line computation. These solutions are theoretically attractive; however, their practical contribution has not been proved, especially in coping with intrazonal trips omission or in overcoming the spatial aggregation bias. Furthermore, with increasing computation capacities, it becomes possible to design models with barely no limitation on the number of zones using more efficient implementation of the DUE like that of the Origin-Based Assignment (Inoue and Maruyama, 2012).

The link subdivision method consists in subdividing zones using network links. This method has been proposed by Horowitz (2001) as a new assignment strategy to increase the spatial precision of traffic assignment models. Practically, each network intersection is assigned a service area corresponding to the Voronoi polygon around this intersection. In this manner, each Traffic Analysis Zone (TAZ) is subdivided into different intersection sectors. A new trip table is derived from the original one in proportion with the relative area of each subzone, i.e. sector. By doing so, intrazonal demand can be distributed between subzones. The resulting trip table is assigned using intersection areas as zones and network intersections as centroids. In this case, centroid connectors are not used since zone centroids are also network nodes.

This new assignment method is tested in two case studies: Fredericton, New Brunswick and Racine, Wisconsin in the US (Horowitz, 2001). According to the author, these case studies are realistic enough to reasonably test the new method and simple enough to be easily manageable. The new assignment strategy is compared to the conventional assignment method, namely: aggregate assignment with no subdivision. Validation indicators are constructed using traffic ground counts. Results from both case studies are not conclusive. The contribution of the new assignment strategy is not clear when compared to the standard one. In some cases, the new strategy fails to reproduce ground counts in comparison with the standard method. The author suggests further research and tests to assess the contribution of this new assignment strategy.

In another similar research design, Mann (2002) develops a new assignment strategy based on zoning subdivision around centroid connectors instead of links. Each TAZ with more than one connector link, is subdivided into different subzones. Each subzone corresponds to the catchment area of its corresponding centroid connector. Each subzone receives a proportion of travel demand according to a predefined criterion (uniform distribution, proportional to land-use, or proportional to intrazonal network length). If a zone has only one connector, the modeler can add manually two or more 
connectors and re-apply the above steps to create subzones.

This method was primarily designed to correct for the fictitious congestion problem induced by overloading trips on few centroid connectors (Sean Qian and Zhang, 2012). In some cases where trip attractions or productions are high and only few connectors are used to convey travel demand, an overload around connection nodes appears and biases assignment outcomes. This situation is detrimental to the modeling of route choice and to the estimation of travel times. In this regard, subdivision around centroid connectors is a practical assignment framework to distribute travel demand between different connectors and to avoid the fictitious congestion problem. This method can also be used to assign intrazonal trips between subzones. However, Mann (2002) suggests that this option might introduce a bias in the analysis as it may cause the diversion of interzonal trips from arterial roads to local streets. This is because the assignment of intrazonal trips requires the inclusion of local streets in the definition of the network for the sake of consistency (Bovy and Jansen, 1983). For this reason, the author discards local streets and intrazonal trips from his research protocol and gives no conclusion about the contribution of the method regarding intrazonal trips assignment.

Another approach inspired by the Bureau of Public Roads (BPR) in the US is to distribute intrazonal trips to adjoining zones and to perform a standard zone-to-zone assignment (Bureau of Public Roads, 1964). For each zone with an intrazonal travel demand, the destination of half of intrazonal trips is switched from mother-zone to adjoining zones. The other half is distributed the other way, from adjoining zones to the corresponding mother-zone. This method is similar to that used by the BPR to estimate intrazonal travel times needed in gravity models. This is a first-cut method that does not reflect the true route choice mechanisms at play, especially in coarse models. With a coarse zoning for example, this method can make intrazonal trips travel long distances from their original zone to reach adjoining zones and therefore to overestimate intrazonal travel times.

\subsubsection{Supply-side methods}

On the other hand, supply-side methods acknowledge the bias of ignoring intrazonal trips by including the impact of this omission in the definition of the transportation network. Ignoring intrazonal trips in traffic assignment induces a fictitious free-flow bias as demonstrated in Manout and Bonnel (2018). To take into account this bias, supply-side methods act on the definition of network links by reducing their flow capabilities. In this regard, two approaches can be used:

- Network preloading approach.

- Network coding approach.

The preloading approach assigns intrazonal trips to the network using some ad hoc methods (Moeckel and Donnelly, 2009). Intrazonal trips are preloaded on the network and added to flows computed 
by standard assignment models. In a national US-freight model, Moeckel and Donnelly (2009) estimate intrazonal traffic using a raster method. Each network link is assigned an intrazonal traffic proportional to the density of jobs around its nodes. Congested travel times are computed afterwards by adding preloaded intrazonal traffic to the assigned interzonal trips. In this manner, intrazonal trips can have a direct impact on interzonal trips. This is a straightforward method to account for intrazonal trips; nevertheless, this method bears noteworthy shortcomings. Transforming intrazonal demand (aggregate demand) into intrazonal traffic (link flows) is a problem that is better to be solved by an assignment algorithm rather than an approximate method. Furthermore, only the impact of intrazonal traffic on interzonal flows is accounted for; the opposite-direction impact of interzonal on intrazonal traffic is ignored. Moreover, this method seems to be difficult to transfer from one spatial context to another without recalibrating the relation between jobs and intrazonal traffic. Finally, this method has been applied to a national level with a sketch transportation network and seems to be less appropriate for urban modeling. For all these reasons, the current research does not investigate further this approach and focuses exclusively on the coding approach.

The coding approach consists at reducing the capacity or the free-flow speed of network links to counterpart the fictitious free-flow bias induced by the omission of intrazonal trips. Instead of preloading intrazonal traffic on the network, it is possible to translate the effect of their omission by reducing the capacity of the network. On each network link, a reduction factor is applied in proportion to its intrazonal flows. We could not find any paper discussing this method, therefore, this research suggests to investigate the contribution of this assignment strategy.

\subsubsection{Mix methods}

Finally, by mixing both demand-side and supply-side strategies, mix methods come up with new assignment strategies to account for intrazonal trips. One example of this is the subarea focusing method made popular by the Federal Highway Administration (FHWA) of the US Department (DeCorla-Souza and Grubb, 1991; Texas Transportation Institute and State Department of Highways and public transportation, 1988). The focusing method is used to refine the modeling resolution of specific zones by including more spatial and network details than in the rest of zones. Zones are subdivided as in the case of demand-side methods and low road hierarchies are added to the original network description to avoid the fictitious congestion problem (Bovy and Jansen, 1983; Mann, 2002). These methods are popular in sketch planning models where the description of zones and network is coarse. The focusing method allows to simulate detailed projects and plans in an aggregated modeling framework with limited costs. However, the focusing method is often manually carried out to match each project details and needs.

In view of this state-of-the-art on traffic assignment methods and intrazonal trips, no clear-cut conclusions can be drawn about the contribution of these different assignment strategies. On one hand, the contribution of the majority of these methods is yet to be scientifically demonstrated in 
real-world case studies using statistical analysis. On the other hand, a cross-comparison between these methods is yet to be undertaken in order to provide informative recommendations and conclusions. To this end, the current research conducts a comprehensive analysis of different assignment strategies, including demand-side, supply-side, and mix methods. The contribution of each method is assessed in absolute and relative terms and in three different case studies.

\subsection{Objectives}

In our quest to build practical assignment models that tackle more suitably the aggregation bias, different assumptions and design choices are made. These choices are often in line with 4 guidelines:

- Practical models: the first aim of this research is to provide practical recommendations for both researchers and practitioners. A constant trade-off between complexity, accuracy, and costs is made.

- Replicable models: for our recommendations to be useful and used, a special attention is paid to spatial and temporal replicability of our findings. Manual interventions are kept minimal.

- Minimal data requirement: for the sake of practicality and replicability, data requirements are limited to ubiquitous datasets.

- Computationally lightweight procedures.

\subsection{Methods}

This section investigates 4 different methods to assign intrazonal trips. Each method combines a specific definition of the three following components: travel demand, transportation network, and spatial design. The four methods attempt to include, in different ways, intrazonal trips in traffic assignment models. They are first applied to two case studies: (1) a 4 zones 8 links case study, and (2) the Sioux Falls case study. Assignment outcomes from these cases are validated against reference network flows. Most conclusive methods from this validation step are applied to the more complex case study of Lyon in France.

Throughout this section, we use a simple case study of 4 zones and 8 links to illustrate the new assignment strategies (figure 5.1). This simple case compounds 4 zones attached by 8 directed links. Each zone centroid is also a graph node. Travel demand is defined in table 5.1. For the study of intrazonal trips, a new aggregated zoning of the original one is defined (graphic b in figure 5.1). Two zones: 1 and 2 are merged together to produce a new macro-zone 5 . This macro-zone is connected to the transportation network using subzone connection nodes: 1 and 2 . In the sequel of this chapter, links are referred to by their origin and destination nodes: origin $\rightarrow$ destination. 

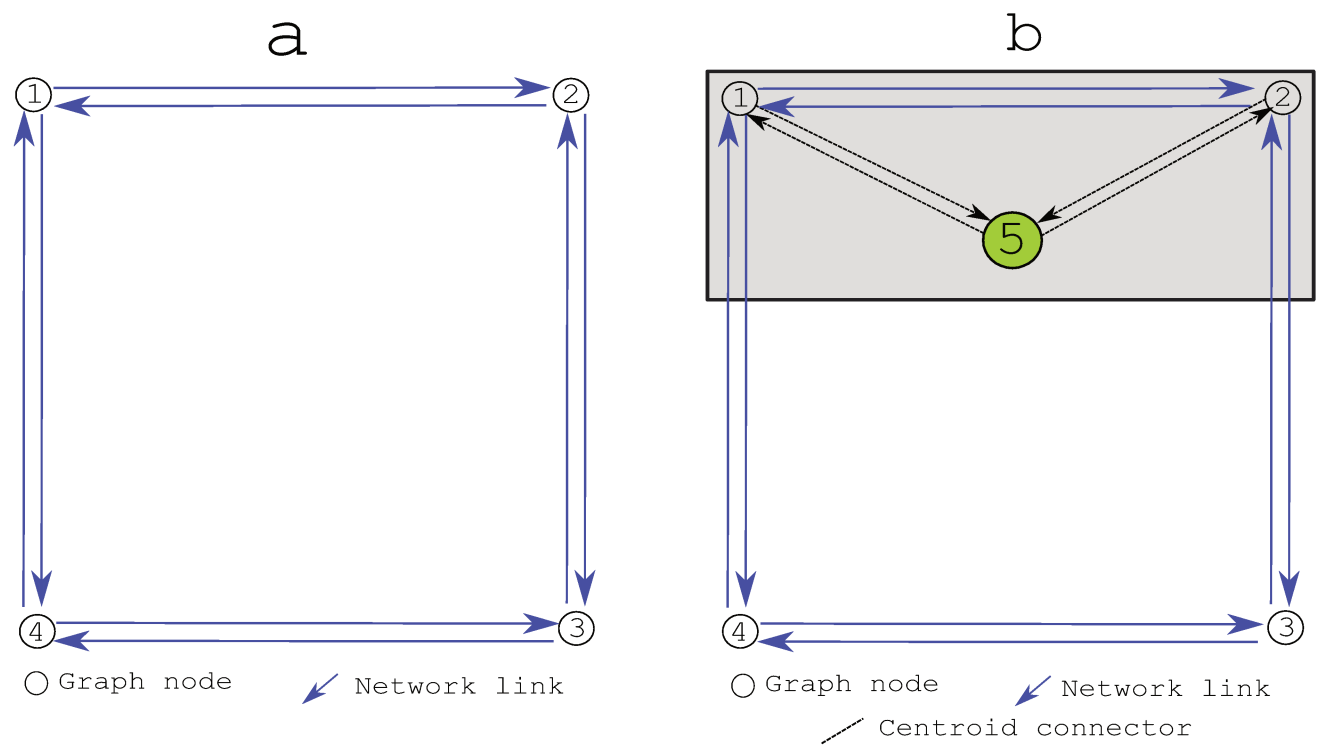

Figure 5.1: First case study: 4 zones, 8 links

Table 5.1: Demand matrix of the simple case study

\begin{tabular}{ccc}
\hline Origin & Destination & Demand \\
\hline 1 & 2 & 50 \\
1 & 4 & 100 \\
2 & 1 & 150 \\
2 & 4 & 250 \\
3 & 1 & 100 \\
3 & 2 & 100 \\
3 & 4 & 100 \\
4 & 3 & 100 \\
\hline
\end{tabular}




\subsubsection{Traffic assignment strategies}

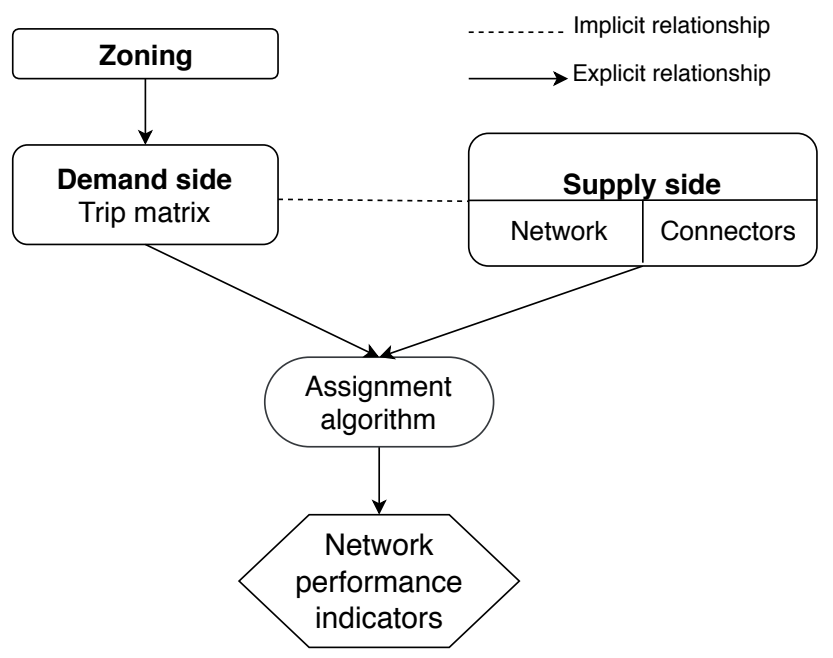

Figure 5.2: A model of traffic assignment models

An assignment strategy is a combination of 5 different components (figure 5.2):

- Zoning: a spatial design.

- Trip table: a travel demand matrix dependent on the zoning.

- Network: a representation (shapes) and a description (characteristics) of a transportation network.

- Centroid connectors: a definition of access and egress links.

- Assignment algorithm: a route choice algorithm.

Each component of the above list can be defined and modeled in various ways. This section explores different combinations of these elements to construct 4 assignment strategies with 6 different approaches to address the spatial aggregation problem in traffic assignment.

Intrazonal trips are a direct consequence of using a discrete description of space. These trips depend on the spatial discretization method, i.e. the spatial design. In each case study, the original zoning is aggregated progressively to produce different zonings with varying patterns of intrazonal trips. For each produced zoning, a corresponding demand matrix is computed. This aggregation procedure is carried out manually for the first two simple case studies and automatically for the more complex Lyon case study.

As far as possible, the transportation network is modeled in detail. All road links are included, from highway roads to local streets. Link characteristics are described using free-flow travel times in seconds, link flow capacity in vehicle/hour, and a Volume-Delay Function describing the relation between travel times and flow. Centroid connectors are defined using two different approaches to 
complete the definition of the network. For lack of time, the author could not adapt the previous definition of transit connectors, developed in chapter 3, to the case of car connectors.

The Deterministic User Equilibrium (DUE) is opted for as an assignment framework. The DUE builds on the first principle of Wardrop (1952) and was first formulated by Beckmann et al. (1956). Even if this assignment principle is deemed to be less accurate at reproducing individual route choices compared to the Stochastic User Equilibrium (SUE) for example (Sheffi, 1984), the DUE

method is easy to implement, tractable, computationally lightweight, and above all deterministic. A Frank-Wolf implementation of this algorithm is used (Frank and Wolfe, 1956) with a maximum number of iterations set at 100 and a stopping criterion, i.e. relative gap, set at $10^{-4}$. Most assignment algorithms use these convergence criteria (for more details, see Bovy and Jansen (1983) for the definition of the relative gap, and Rose et al. (1988) for a comprehensive comparison between convergence criteria). The algorithm stops when at least one of the aforementioned criteria is met.

\subsubsection{Method 1: the standard assignment model}

This strategy refers to the conventional assignment model as encountered in the majority of research papers and transportation studies. This method ignores intrazonal trips by assigning only the interzonal part of the travel demand. This assignment method is included in our research to benchmark the relative contribution of new assignment strategies.

In the standard method, it is a common practice to set an infinite capacity on centroid connectors. This means that connectors' travel times are constant and independent of traffic flows. In the current research, an infinite capacity is set on centroid connectors. Travel demand is aggregated and intrazonal trips discarded. The outcomes of the standard assignment are referred to as the standard results.

The practice of setting an infinite capacity on connectors is however questionable since it may induce errors in route choice modeling. This bias has already been brought to light in chapter 4 as the rerouting problem. No solution has been proposed in chapter 4 to address this problem or at least to evaluate the extent of its impact on assignment results. The next assignment method addresses, to some degree, the rerouting problem by challenging the infinite capacity assumption.

\subsubsection{Method 2: standard assignment method with a finite capacity on con- nectors}

This method is similar to the standard assignment apart from the definition of centroid connectors. In the standard method, the capacity of connector links is supposed to be infinite and travel times to be constant. These assumptions are relaxed in the current method. Travel times are no longer 
constant but follow a Volume-Delay Function (VDF) of type BPR $2^{3}$. Given this function, travel times on connectors increase with their saturation levels. The saturation level of a link is the ratio between its flow and its capacity over a period of time that is often 1 hour. In the case of centroid connectors, these artificial links have no capacity to compute their saturation levels. The current method addresses the specific question of assigning a capacity to centroid connectors.

Centroid connectors are artificial links introduced in the definition of the network to allow trips, produced and attracted by zone centroids, to access and egress the network. Since these links have no real counterpart, it is not straightforward to assign a capacity to them. In fact, in transportation modeling, the capacity of a link refers to the maximum sustainable car flow expressed in vehicles/hour $(v e h / h)$ this link can convey in prevailing conditions (Transportation Research Board, National Research Council, 2000). Given this definition, the capacity of a link is tightly dependent on its physical characteristics: geometry, number of lanes, speed limit, crossing characteristics. Centroid connectors have no physical characteristics to deduce the capacity from. Nevertheless, these links have a functional role and their capacity can be defined accordingly.

Here, we define the capacity of a centroid connector as proportional to trip attractions and productions of the zone centroid attached to this connector. For this purpose, two approaches are used: uniform and original definitions (eq. 5.1, eq. 5.2).

\subsection{Approach 1: standard assignment with a uniform capacity on connec- tors}

This first approach is straightforward. In the absence of any intrazonal information, all centroid connectors of a zone $Z$ are supposed to have the same capacity independently of their connection nodes $N_{i}$. This assumption reduces to some extent the bias of an infinite capacity but it is still an ad hoc approach. The capacity of a centroid connector attaching node $N_{i}$ and zone $Z$ is expressed in eq. 5.1:

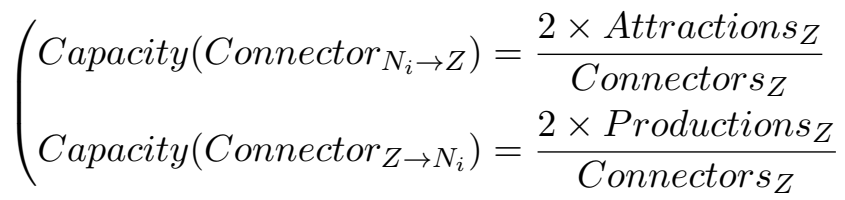

Where Attractions $s_{Z}$ and Productions $s_{Z}$ are, respectively, trip attractions and productions of zone $Z$ excluding its intrazonal trips. Connectors $s_{Z}$ is the number of directed connectors of zone $Z$. This method is said to be uniform as the capacity of connectors is only dependent on the zone centroid and not the connection node.

\footnotetext{
${ }^{3} \mathrm{BPR}$ functions were first introduced by the Bureau of Public Roads in the US to model the evolution of travel times under congested conditions (Bureau of Public Roads, 1964). Here, we use a BPR function of type 2 that is more sensitive towards congestion. Other functions may as well be used (see Branston (1976) and Spiess (1990) for a review on the subject).
} 
When applied to the example in figure 5.1 and table 5.1, the capacity of centroid connectors is given in table 5.2 .

Table 5.2: Uniform capacity of centroid connectors of the simple case study according to method 2.1

\begin{tabular}{ccc}
\hline From node & To node & Capacity (veh/hour) \\
\hline 1 & 5 & 100 \\
2 & 5 & 100 \\
5 & 1 & 175 \\
5 & 2 & 175 \\
\hline
\end{tabular}

This method can be refined by adding more intrazonal data to the definition of connectors. The next approach introduces a more sophisticated definition of connectors using intrazonal data. This approach is used as a reference to assess the bias introduced by the uniform distribution.

\subsection{Approach 2: standard assignment with original capacity on connectors}

Instead of using a uniform distribution, the second approach suggests to use intrazonal data of subzones. Macro-zones are designed to have the same connection nodes as their subzones. The capacity of a centroid connector attaching a macro-zone $Z$ to a connection node of a subzone $N_{i}$ can be defined on the basis of attractions and productions of $N_{i}$ instead of global macro-zone characteristics (eq. 5.2):

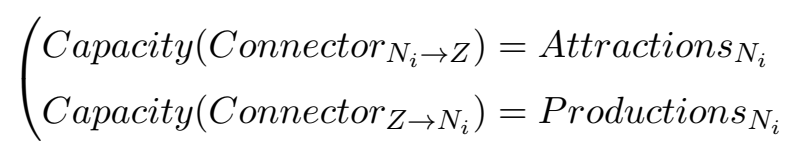

Where Attractions $_{N_{i}}$ and Productions $N_{N_{i}}$ are, respectively, trip attractions and productions of subzone $N_{i}$. This method is said to be original as the capacity of connectors derives from the original distribution of travel demand between subzones.

When applied to the example in figure 5.1 and table 5.1, the capacity of centroid connectors is given in table 5.3.

Table 5.3: Original capacity of centroid connectors of the simple case study according to method 2.2

\begin{tabular}{ccc}
\hline From node & To node & Capacity (veh/hour) \\
\hline 1 & 5 & 100
\end{tabular}




\begin{tabular}{ccc}
\hline From node & To node & Capacity (veh/hour) \\
\hline 2 & 5 & 100 \\
5 & 1 & 100 \\
5 & 2 & 250 \\
\hline
\end{tabular}

Both methods: 1 and 2 do not address the problem of ignoring intrazonal trips, but rather the question of flow rerouting. In the sequel of this chapter, the capacity of centroid connectors is defined according to one of these two approaches.

\subsubsection{Method 3: standard assignment with capacity reduction of intrazonal links}

This assignment strategy belongs to supply-side methods. In real world, trips that are considered intrazonal, use the transportation network and take up some of its capacity. Standard assignment methods do not take into consideration this consumed capacity and therefore introduce a fictitious free-flow bias in assignment outcomes. In order to include the impact of intrazonal trips, this method consists at reducing the capacity of the transportation network proportionally to conveyed intrazonal trips.

The impact of these ignored trips is included in the definition of the capacity of intrazonal links. An intrazonal link is a link that is attached to a centroid connector. In figure 5.1, links $1 \rightarrow 2$, $2 \rightarrow 1,2 \rightarrow 3,3 \rightarrow 2,1 \rightarrow 4$, and $4 \rightarrow 1$ are intrazonal links of zone 5 . Links $4 \rightarrow 3$ and $3 \rightarrow 4$ are extra-zonal links. On each intrazonal link, a capacity reduction factor is applied. This factor is proportional to the amount of its intrazonal flows. However, in transportation models, this information is not available since intrazonal trips are not assigned. For this reason, two assumptions are made:

1. All intrazonal links are supposed to convey a part of intrazonal demand proportionally to their capacity.

2. For each intrazonal link, the reduction factor $(\mathrm{RF})$ is supposed to be proportional to the capacity of the link and the total intrazonal trips of the zone (eq. 5.3).

$$
\left\{\begin{array}{l}
R F_{Z}=\frac{\text { Intrazonal_Trips }}{\text { old }_{Z}} \\
\sum_{k}\left(C_{\text {link }_{k}}\right) \\
C_{\text {link }}^{\text {new }}=\left(1-R F_{Z}\right) \times C_{\text {link }_{a}}^{\text {old }} \\
\sum_{k} C_{\text {link }}^{\text {new }}=\sum_{k} C_{\text {link }}^{\text {old }}-\text { Intrazonal_Trips } \\
\end{array}\right.
$$


Where $C_{\text {link }_{a}}^{\text {new }}, C_{\text {link }}^{\text {old }}$ are, respectively, the new and old capacities of link a. $\operatorname{lin} k_{a}$ is an intrazonal link of zone $Z$. To avoid negative or unrealistic low values of $C_{\text {link }_{a}}$, the minimum value of this quantity is set to $10 \mathrm{veh} / \mathrm{h}$. The choice of this threshold is arbitrary.

In method 3, centroid connectors are defined according to method 2.1, namely: a finite and uniform capacity definition.

When applied to the example in figure 5.1 and table 5.1, the new capacity of intrazonal links is set to $67 \mathrm{veh} / \mathrm{h}$ instead of $100 \mathrm{veh} / \mathrm{h}$.

This method reduces the capacity of intrazonal links to account for the impact of ignoring intrazonal trips. Given this reduction, travel times of intrazonal links increase to counteract the free-flow bias. However, this method is more appropriate to reproduce travel times than traffic flows. In fact, this method fails, by design, to reproduce observed link flows as intrazonal trips are still not assigned to the network.

\subsubsection{Method 4: subdivision of zones}

In order to assign intrazonal trips, the trip table should, first, contain these trips, and second, differentiate between their origins and destinations. In standard traffic assignment models, these conditions are not met. One solution to this problem is to divide zones into subzones (Daganzo, 1980b; Horowitz, 2001; Mann, 2002). In this manner, intrazonal trips can be assigned to different destinations inside their mother-zone. In the current research, two approaches are used to carry out this subdivision.

\subsection{Approach 1: subdivision of zones using a uniform distribution of in- trazonal demand}

Each macro-zone is divided into its original subzones. The trip demand matrix of the new subdivision is a combination of the aggregate trip table and the original one. Intrazonal trips of macro-zones are distributed uniformly between subzones.

When applied to the first case study where zones 1 and 2 are aggregated to form macro-zone 5 (table 5.1), this method produces a new trip table 5.4. Intrazonal trips of zone 5 (200 trips) are distributed uniformly between its subzones: 1 and 2 .

Table 5.4: Trip demand matrix according to method 4.1

\begin{tabular}{ccc}
\hline Origin & Destination & Demand \\
\hline 1 & 2 & 100 \\
2 & 1 & 100 \\
3 & 4 & 100
\end{tabular}




\begin{tabular}{ccc}
\hline Origin & Destination & Demand \\
\hline 3 & 5 & 200 \\
4 & 3 & 100 \\
5 & 4 & 350 \\
\hline
\end{tabular}

To control for the rerouting problem, centroid connectors are defined according to method 2.1, namely: finite and uniform capacity of connectors.

\subsection{Second approach: subdivision of zones using the original distribution of intrazonal demand}

This approach is similar to the previous one apart from the distribution of intrazonal trips and the definition of connectors' capacity. Intrazonal trips of macro-zones are distributed between subzones as in the original trip table (table 5.5). Interzonal trips are aggregated as in the standard assignment. This method is only used to assess the contribution of the first approach and the bias from using an ad hoc distribution method.

Table 5.5: Trip demand matrix according to method 4.2

\begin{tabular}{ccc}
\hline Origin & Destination & Demand \\
\hline 1 & 2 & 50 \\
2 & 1 & 150 \\
3 & 4 & 100 \\
3 & 5 & 200 \\
4 & 3 & 100 \\
5 & 4 & 350 \\
\hline
\end{tabular}

To control for the rerouting problem, centroid connectors are defined according to method 2.2, namely: finite and original capacity of connectors.

Both approaches of method 4 allow for the conservation of total travel demand: whatever spatial aggregation is performed, aggregated and original trip tables have the same total of assignable trips. No omission occurs with spatial aggregation.

\subsubsection{Synthesis}

Tables 5.6 sums up the four assignment strategies. 
Table 5.6: Synthetic definition of the four assignment methods

\begin{tabular}{lrrrr}
\hline & Method 1 & Method 2 & Method 3 & Method 4 \\
\hline Travel Demand & Aggregated & Aggregated & Aggregated & Aggregated + Detailed \\
Network & Original & Original & Capacity reduction & Original \\
Connectors & Infinite & Finite & Finite & Finite \\
\hline
\end{tabular}

\subsection{Case studies}

The contribution of the aforementioned methods is assessed in three case studies, beginning with the simple case study of 4 zones 8 links.

\subsubsection{Simple case study: a 4 zones 8 links example}

This is a sketch case study with 4 zones and 8 links (sub-figure $a$ in figure 5.1). All network links have the same characteristics: $100 \mathrm{veh} / \mathrm{h}$ capacity and $10 \mathrm{~s}$ free-flow travel time. The Travel demand is described in table 5.1.

On the basis of this original case study, an aggregated zoning is constructed by merging zones 1 and 2 to produce zone 5 . The new macro-zone is connected to the transportation network using subzone connection nodes 1 and 2 (sub-figure $b$ in figure 5.1).

\subsubsection{Sioux Falls case study}

The Sioux Falls case study is a sketch network of the Sioux Falls city in South Dakota, USA ${ }^{4}$. This case study is widely used among transportation scientists for validation and testing purposes (Abdulaal and LeBlanc, 1979; Bar-Gera et al., 2013; Friesz et al., 1992; Luathep et al., 2011; Meng et al., 2001; Wang et al., 2013). The transportation network consists in a sketch graph of 76 directed links and 24 nodes (figure 5.3). Each network node is also a zone centroid. Thus, the spatial zoning of the Sioux Falls case study compounds 24 zones that are attached directly to the network, i.e. centroid connectors have a zero length. The trip table has 360,600 car trips.

The Sioux Falls trip matrix does not include intrazonal trips. For the purpose of this research, the original zoning is aggregated to produce intrazonal demand. 5 different spatial designs are prepared to get different aggregation patterns. The rate of intrazonal trips ranges from $2 \%$ to $22 \%$ (table 5.7 ).

\footnotetext{
${ }^{4}$ This case study does not reflect the real transportation network nor the travel demand of Sioux Falls city. Data are available at: https://github.com/bstabler/TransportationNetworks/tree/master/SiouxFalls.
} 


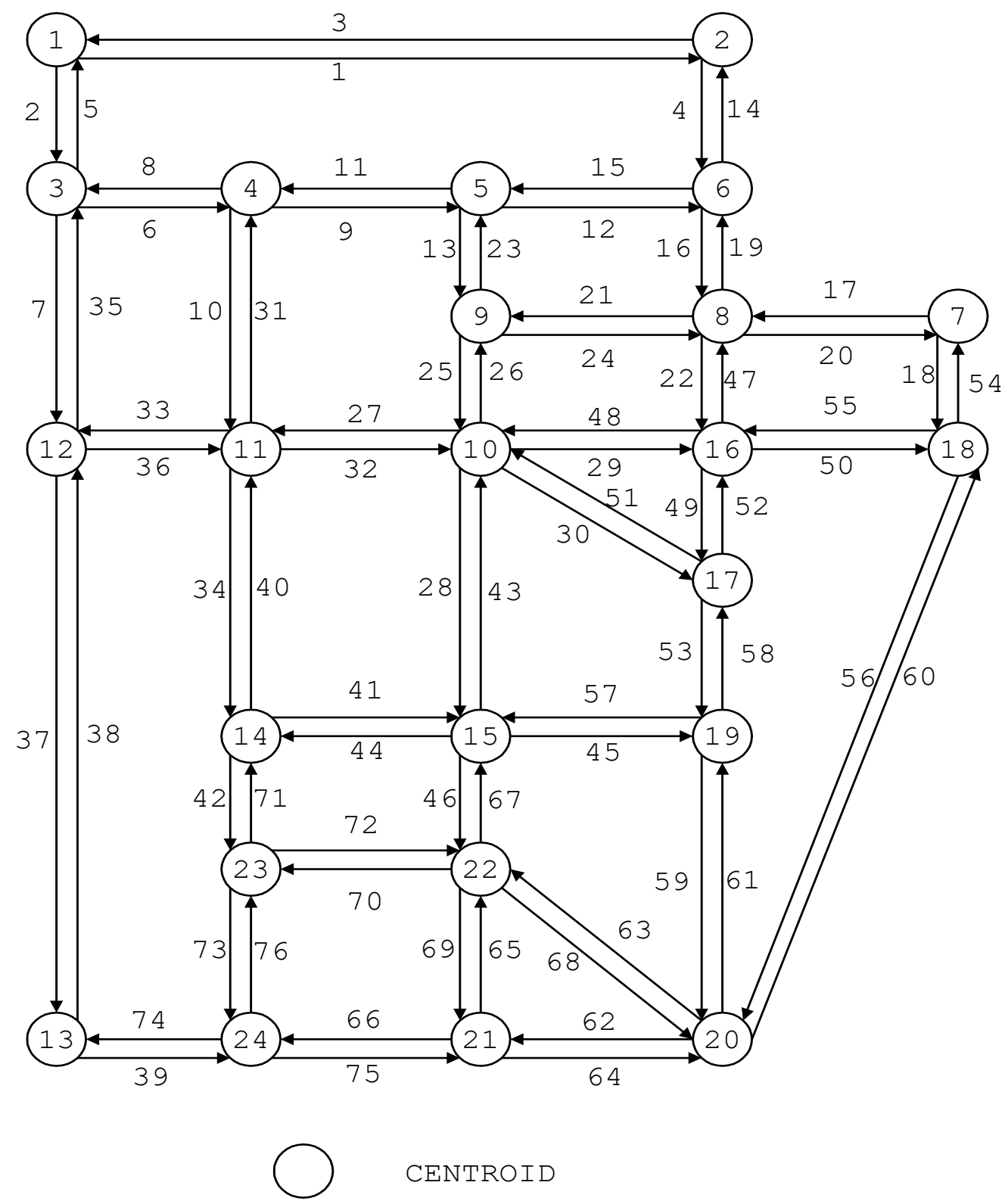

Figure 5.3: Sioux Falls network Wang et al. (2013)

Table 5.7: Aggregated spatial designs of the Sioux Falls case study

\begin{tabular}{cccc}
\hline Zoning & Subzones & Intrazonal trips & Intrazonal trips rate $(\%)$ \\
\hline Zoning 1 & $1,2,3,4,5,6$ & 7,400 & 2.05 \\
Zoning 2 & $1,3,4,11,12,13,14,23,24$ & 41,600 & 11.54 \\
Zoning 3 & $1,2,3,4,5,6,7,8,9,10$ & 51,000 & 14.14 \\
Zoning 4 & $13,14,15,19,20,21,22,23,24$ & 64,700 & 17.94 \\
Zoning 5 & $2,5,6,7,8,9,10,16,17,18$ & 80,800 & 22.41 \\
\hline
\end{tabular}


For zoning 1 for example, zones 1, 2, 3, 4, 5, and 6 are merged to form a new macro-zone (table 5.7). The same goes for the rest of zonings. For this sketch case study, a simplistic aggregation method based on contiguity is used. Other criteria of aggregation are used in the more sophisticated case study of Lyon.

Both case studies: the simple and the Sioux Falls case study, are used to identify most conclusive assignment strategies. Most promising methods are further investigated in the case study of Lyon.

\subsubsection{Lyon case study}

The Lyon case study is drawn from a strategic transportation model of the urban area of Lyon (figure 5.4). In population terms, the urban area of Lyon is the most second largest in France. The traffic model has been developed to appraise macroscopic urban planning schemes. It has been validated against traffic counts and observed travel times.

The original spatial zoning of this model is based on the IRIS administrative division (Insee, 2017). Each IRIS zone contains at least 2,000 inhabitants. The urban area of Lyon contains 777 such zones (figure 5.4). 


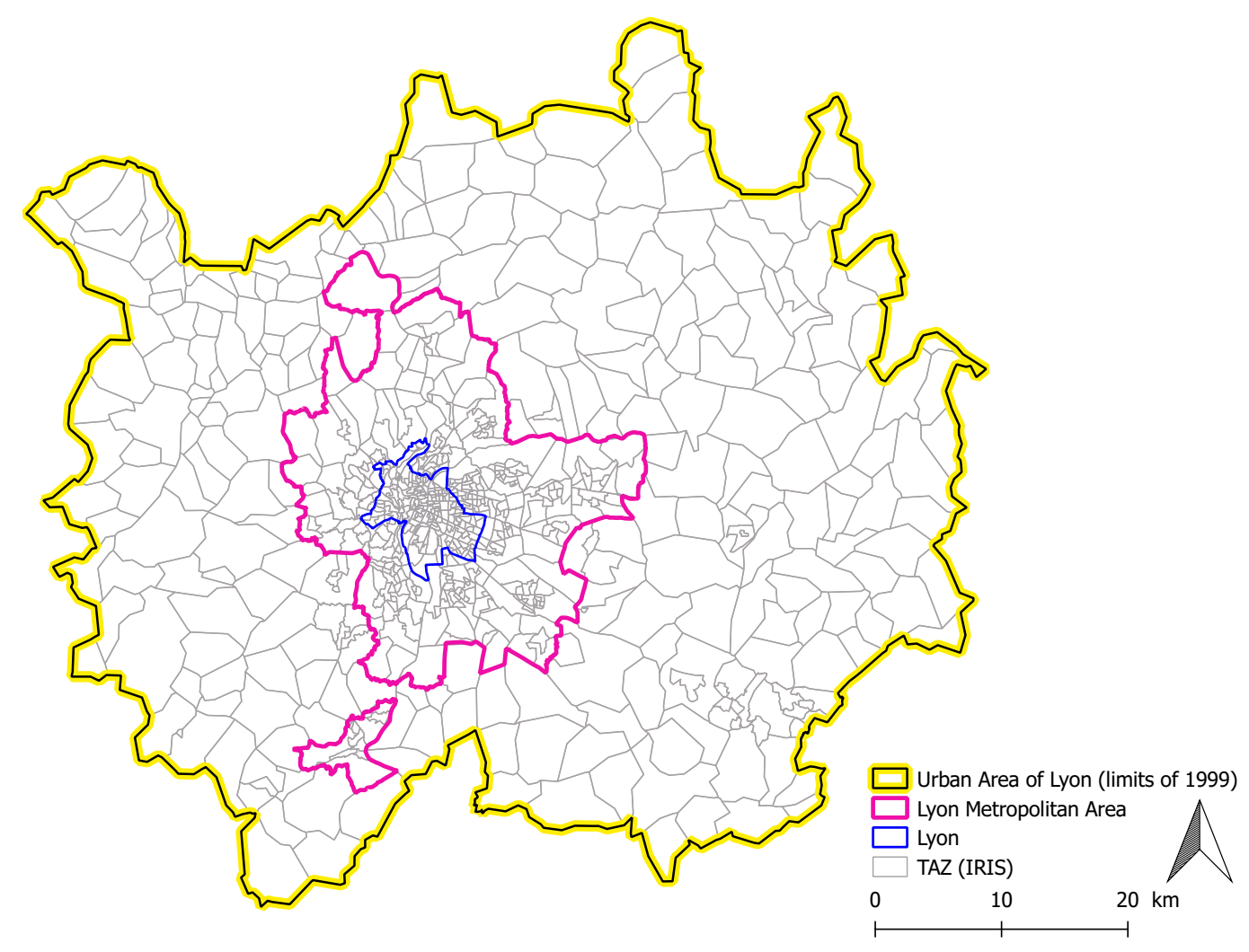

Figure 5.4: Original zoning of the case study of Lyon

\subsubsection{Travel demand}

The trip table describes travel demand at the evening rush hour (5-6pm) of an ordinary working day of 2015. This table is deduced from an update of the Households Travel Survey (HTS) of 2006 (Agence d'Urbanisme de Lyon, 2006). The demand matrix has been updated and validated to match 2015 traffic counts. Total travel demand is about 314,483 car trips, of which 8,028 trips or $2.6 \%$ are intrazonal.

\subsubsection{Network}

The transportation network of Lyon is finely modeled: 48,641 nodes and 113,690 edges. GPS data from Navteq datasets are used to describe link characteristics. Network links, from highway roads to local streets, are included in the description of the network. For modeling purposes, the network is divided to 5 groups according to their functional class (figure 5.5, table 5.8). Each road hierarchy contains links that share similar road characteristics (free-flow speed, capacity, number of lanes, functional role). 
Table 5.8: The hierarchy of the transportation network in the case of Lyon. Average capacity and speed are weighted by the length of links

\begin{tabular}{lccccc}
\hline $\begin{array}{l}\text { Hierarchy } \\
\text { name }\end{array}$ & $\begin{array}{c}\text { Road } \\
\text { category }\end{array}$ & $\begin{array}{c}\text { Number of } \\
\text { links }\end{array}$ & $\begin{array}{c}\text { Cumulative } \\
\text { length }(\mathrm{km})\end{array}$ & $\begin{array}{c}\text { Average Capacity } \\
\text { (veh/hour) }\end{array}$ & $\begin{array}{c}\text { Average } \\
\text { Speed }(\mathrm{km} / \mathrm{h})\end{array}$ \\
\hline $\begin{array}{l}\text { Highways } \\
\text { Arterial roads }\end{array}$ & 1 & 570 & 474 & 4,421 & 113 \\
Major & 3 & 1,926 & 429 & 2,411 & 81 \\
collector & & 13,285 & 2,174 & 1,573 & 71 \\
roads & & & & & 68 \\
Minor & 4 & 24,610 & 4,173 & 1,335 & \\
collector & & & & & 56 \\
roads & & & & & \\
Local streets & 5 & 69,434 & 13,372 & 1,158 & \\
All network & All & 109,825 & 20,622 & & \\
& network & & & & \\
\hline
\end{tabular}

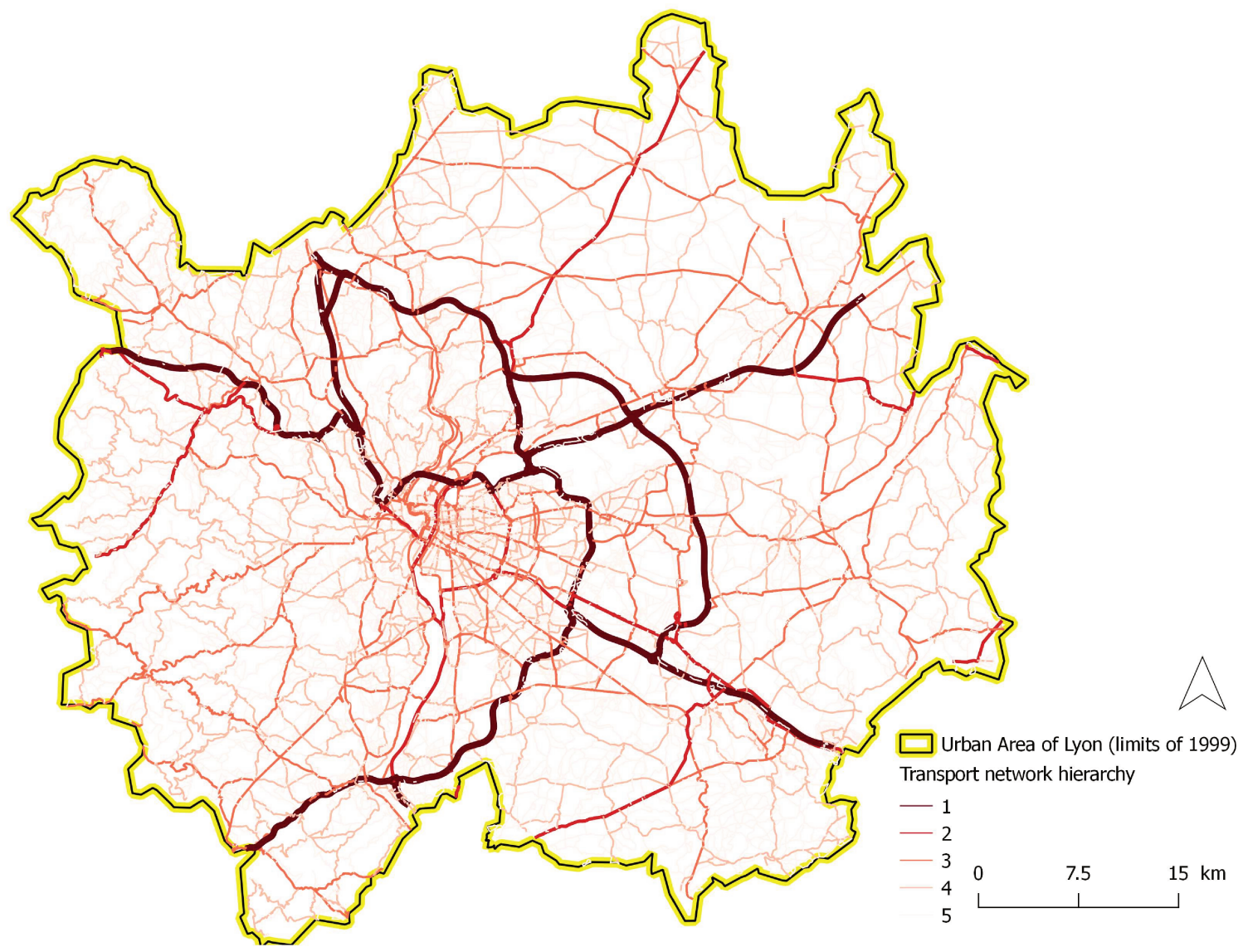

Figure 5.5: The transportation network of Lyon 


\subsubsection{Zoning}

The case study of Lyon has already been used to examine the impact of ignoring intrazonal trips in assignment results using a stochastic approach in chapter 4. In the current research, we adopt the same stochastic approach to assess, statistically, the contribution of each new assignment strategy. For this purpose, 700 spatial designs are defined for the urban area of Lyon. Each zoning is constructed in a stochastic manner as described in chapter 4. 7 levels of aggregation are retained: from 100 to 700 zones (multiples of 100). For each level of aggregation, 100 variants are computed in order to avoid any sampling bias. In fine, 700 zonings are designed with an intrazonal trips rate ranging from $0.05 \%$ to $52 \%$ of total demand (figure 5.6).

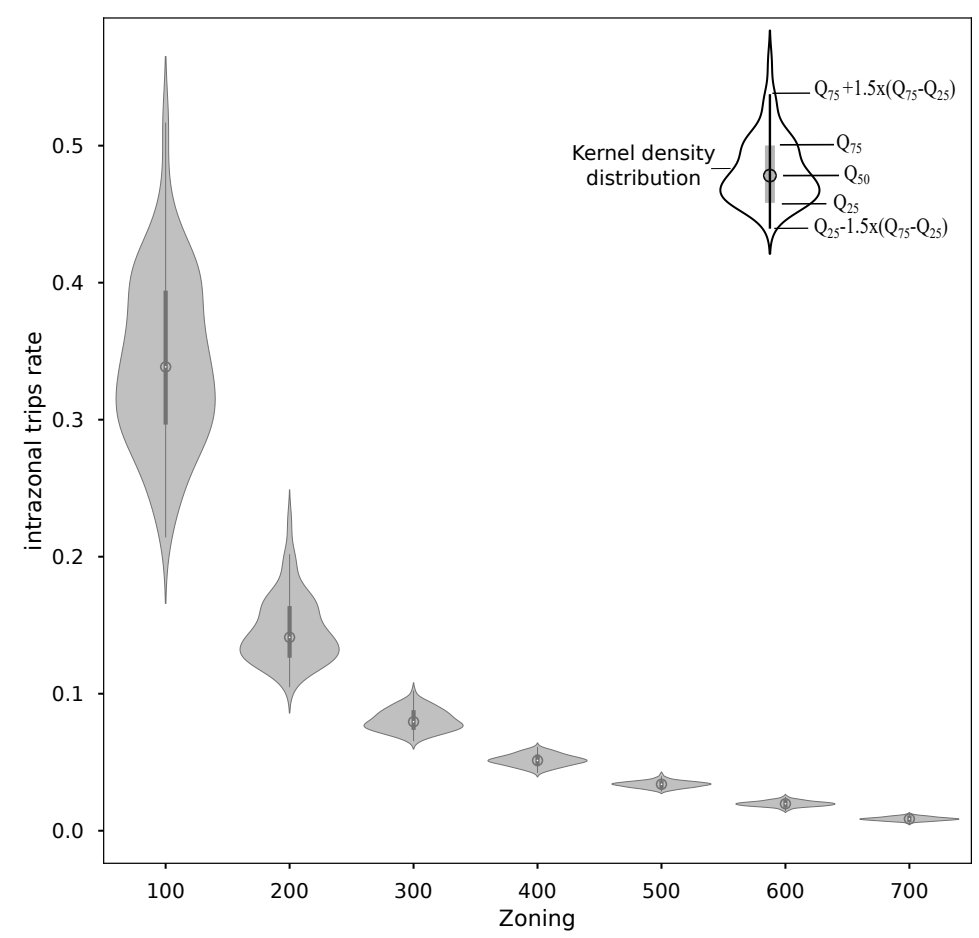

Figure 5.6: Distribution of the rate of intrazonal trips according to the aggregation level

\subsection{Results}

In this section, the absolute and relative contribution of each traffic assignment strategy are examined in three case studies. The contribution of a method is defined as its capacity to reproduce accurately original network flows produced by the original dataset before aggregation. For each case study, original network flows are computed using the original zoning before aggregation. These flows are referred to as reference/original flows or observations (i.e. Obs). After aggregation, assignment results are referred to as simulation results (i.e. Sim). Simulation results are compared to reference 
results using various measures: Relative Difference (RD) (eq. 5.4), Percent Root Mean Square Error (PRMSE) (eq. 5.5), and Geoffrey E. Havers' (GEH) indicator (eq. 5.6).

$$
R D=\frac{S i m-O b s}{O b s} \times 100
$$

The relative difference indicator or percent change is a simple distance measure between observation and simulation results. A positive RD value means an overestimation of flows; a negative sign depicts an underestimation of flows. In some cases, we use the Absolute Relative Difference indicator (ARD) which is the absolute value of RD.

$$
\left\{\begin{array}{l}
R M S E=\sqrt{\frac{\sum_{i}^{n}\left(O b s_{i}-S i m_{i}\right)^{2}}{n}} \\
P R M S E=\frac{R M S E}{\frac{\sum_{i}^{n} O b s_{i}}{n}}
\end{array}\right.
$$

The PRMSE (Percent Root Mean Square Error) is an indicator of deviation. It is widely used by transport modelers in the calibration and validation of operational transport models. The PRMSE indicator is similar to a coefficient of variation. The PRMSE is a relative goodness-of-fit measure that is better to compute for homogeneous data, i.e. data of the same magnitude. Therefore this indicator is computed for each road category. It summarizes in one indicator the accuracy with which original results are reproduced. Accurate methods should have a low PRMSE indicator and vice versa. No absolute threshold values or recommendations are available to compare the PRMSE indicator to. Consequently, this measure is used to compare methods to each other. In this regard, when a method has a lower PRMSE value than another, this method is said to be more accurate in reproducing reference values than the other method.

$$
G E H_{i}=\sqrt{\frac{2 \times\left(O b s_{i}-S_{i m}\right)^{2}}{O b s_{i}+S_{i m}}}
$$

The GEH indicator is another measure of goodness-of-fit. Contrary to the PRMSE, this indicator is computed for each network link to compare reference (original link flows in our case) to simulated link flows with an emphasis on larger than smaller flows. For this reason, this indicator is more often used in microsimulation models than strategic models. British modeling standards recommend strongly the use of this indicator in the calibration and validation of operational transport models (Great Britain and Highways Agency, 1996; Smith and Blewitt, 2010). In this regard, the British Design Manual for Roads and Bridges (DMRB), a widely used practical transport manual, suggests 
some recommendations for operational models to be valid ${ }^{5}$ (table 5.9) (Great Britain and Highways Agency, 1996).

Table 5.9: GEH recommendations (Great Britain and Highways Agency, 1996; Smith and Blewitt, 2010)

\begin{tabular}{ll}
\hline Value & Recommendation \\
\hline GEH less than 5 & Acceptable fit, probably OK. \\
GEH between 5 and 10 & Caution: possible model error or bad data. \\
GEH greater than 10 & Warning: high probability of modeling error or bad data \\
\hline
\end{tabular}

\subsubsection{First case study: the 4 zones 8 links example}

\subsubsection{Original case study}

Before the aggregation of zones 1 and 2, traffic assignment of original travel demand produces the following flows (table 5.10):

Table 5.10: Simple case study: original assignment results

\begin{tabular}{cccc}
\hline From node & To node & Capacity (veh/hour) & Flows (veh/hour) \\
\hline 1 & 2 & 100 & 50 \\
1 & 4 & 100 & 190 \\
2 & 1 & 100 & 297 \\
2 & 3 & 100 & 161 \\
3 & 2 & 100 & 158 \\
3 & 4 & 100 & 303 \\
4 & 1 & 100 & 43 \\
4 & 3 & 100 & 100 \\
\hline
\end{tabular}

Flows in table 5.10 can be decomposed as follows:

\footnotetext{
${ }^{5}$ In our research, the emphasis is more on the relative contribution than the validity of models. That is why accuracy is defined as the capacity of reproducing original link flows rather than ground counts. In this regard, GEH recommendations might be arbitrary for our research since no ground counts are available. Furthermore, the current research does not seek to calibrate nor to validate a transportation model but rather to study the impact of aggregation on assignment outcomes. These recommendations are therefore used for informative purposes only.
} 
- Link $1 \rightarrow 2: 50 \approx 50_{1 \rightarrow 2}$, i.e. link $1 \rightarrow 2$ conveys the total demand between zones 1 and 2 .

- Link $1 \rightarrow 4: 190 \approx 100_{1 \rightarrow 4}+36 \% \times 250_{2 \rightarrow 4}$

- $\operatorname{Link} 2 \rightarrow 1: 297 \approx 150_{2 \rightarrow 1}+36 \% \times 250_{2 \rightarrow 4}+58 \% \times 100_{3 \rightarrow 1}$

- Link $2 \rightarrow 3: 160 \approx(1-36 \%) \times 250_{2 \rightarrow 4}$

- Link $3 \rightarrow 2: 158 \approx 100_{3 \rightarrow 2}+58 \% \times 100_{3 \rightarrow 1}$

- Link $3 \rightarrow 4$ : $303 \approx 100_{3 \rightarrow 4}+(1-58 \%) \times 100_{3 \rightarrow 1}+(1-36 \%) \times 250_{2 \rightarrow 4}$

- Link $4 \rightarrow 1: 43 \approx(1-58 \%) \times 100_{3 \rightarrow 1}$

- $\operatorname{Link} 4 \rightarrow 3: 100 \approx 100_{4 \rightarrow 3}$

Zones 1 and 2 are merged to form a new zone with a new distribution of intrazonal trips. Six different assignment approaches are compared to the original assignment results.

\subsubsection{Method 1: standard assignment method with an infinite capacity on connectors}

With comparison to the original assignment results above, method 1 produces a different distribution of network flows (table 5.11). All network links are impacted by aggregation and especially intrazonal links $1 \rightarrow 2$ and $2 \rightarrow 1$. The impact of intrazonal trips omission on these links is straightforward: these links have the highest error rates (RD and GEH). Furthermore, the standard method is found to underestimate link flows on all links except links $1 \rightarrow 4$ and $3 \rightarrow 2$.

Beyond the problem of ignoring intrazonal demand, another issue, dubbed the rerouting problem, emerges. With aggregation, travel demand of the new created zone 5 can access to the network using both connectors $5 \rightarrow 1$ and $5 \rightarrow 2$. This results in a diversion or rerouting of flows from link $2 \rightarrow 1$. With new connector links, new shortest routes are available to trips. In this regard, demand from zone 5 to 4 can access directly to its final destination using links $5 \rightarrow 1 \rightarrow 4$. After aggregation, $54 \%$ of travel demand between zones 2 and 4 uses the link $1 \rightarrow 4$. This proportion was of $36 \%$ before aggregation. The same goes for travel demand from zones 3 to $5.98 \%$ of travel demand between these zones uses the shortest route $3 \rightarrow 2 \rightarrow 5$ whereas this proportion is of $57 \%$ with the original assignment. These new distributions of travel demand are principally due to the definition of centroid connectors. To this end, subsequent methods restrain the capacity on centroid connectors to control for this issue.

Table 5.11: Simple case: assignment results of method 1. Reference flows of connector links 5-1 and 5-2 are Not Available (NA)

\begin{tabular}{ccccccc}
\hline $\begin{array}{c}\text { From } \\
\text { node }\end{array}$ & $\begin{array}{c}\text { To } \\
\text { node }\end{array}$ & $\begin{array}{c}\text { Capacity } \\
\text { (veh/hour) }\end{array}$ & $\begin{array}{c}\text { Flows } \\
\text { (veh/hour) }\end{array}$ & $\begin{array}{c}\text { Reference flows } \\
\text { (veh/hour) }\end{array}$ & $\begin{array}{c}\text { RD } \\
(\%)\end{array}$ & GEH \\
\hline 1 & 2 & 100 & 0 & 50 & -100 & 10 \\
1 & 4 & 100 & 235 & 190 & 24 & 3 \\
1 & 5 & $+\infty$ & 2 & NA & NA & NA
\end{tabular}




\begin{tabular}{ccccccc}
\hline $\begin{array}{c}\text { From } \\
\text { node }\end{array}$ & $\begin{array}{c}\text { To } \\
\text { node }\end{array}$ & $\begin{array}{c}\text { Capacity } \\
\text { (veh/hour) }\end{array}$ & $\begin{array}{c}\text { Flows } \\
\text { (veh/hour) }\end{array}$ & $\begin{array}{c}\text { Reference flows } \\
\text { (veh/hour) }\end{array}$ & $\begin{array}{c}\text { RD } \\
(\%)\end{array}$ & GEH \\
\hline 2 & 1 & 100 & 0 & 297 & -100 & 24 \\
2 & 3 & 100 & 115 & 161 & -29 & 4 \\
2 & 5 & $+\infty$ & 198 & NA & NA & NA \\
3 & 2 & 100 & 198 & 158 & 26 & 3 \\
3 & 4 & 100 & 217 & 303 & -29 & 5 \\
4 & 1 & 100 & 2 & 43 & -95 & 9 \\
4 & 3 & 100 & 100 & 100 & 0 & 0 \\
5 & 1 & $+\infty$ & 235 & NA & NA & NA \\
5 & 2 & $+\infty$ & 115 & NA & NA & NA \\
\hline
\end{tabular}

Flows in table 5.11 can be decomposed as follows:

- Link $1 \rightarrow 4: 235 \approx 100_{1 \rightarrow 4}+54 \% \times 250_{2 \rightarrow 4}$

- Link $2 \rightarrow 3: 115 \approx(1-54 \%) \times 250_{2 \rightarrow 4}$

- Link $3 \rightarrow 2$ : $198 \approx 100_{3 \rightarrow 2}+98 \% \times 100_{3 \rightarrow 1}$

- Link $3 \rightarrow 4: 217 \approx 100_{3 \rightarrow 4}+(1-98 \%) \times 100_{3 \rightarrow 1}+(1-54 \%) \times 250_{2 \rightarrow 4}$

- Link $4 \rightarrow 1$ : $2 \approx(1-98 \%) \times 100_{3 \rightarrow 1}$

- Link $4 \rightarrow 3: 100 \approx 100_{4 \rightarrow 3}$

- Connector $5 \rightarrow 1: 235 \approx 100_{1 \rightarrow 4}+54 \% \times 250_{2 \rightarrow 4}$

- Connector $5 \rightarrow 2: 115 \approx(1-54 \%) \times 250_{2 \rightarrow 4}$

- Connector $1 \rightarrow 5$ : $2(1-98 \%) \times 100_{3 \rightarrow 1}$

- Connector $2 \rightarrow 5: 198100_{3 \rightarrow 2}+98 \% \times 100_{3 \rightarrow 1}$

\subsubsection{Method 2: standard assignment method with a finite capacity on con- nectors}

Instead of an infinite capacity on centroid connectors $5 \rightarrow 1,5 \rightarrow 2,1 \rightarrow 5,2 \rightarrow 5$, a finite capacity is defined by method 2. Two approaches can be adopted to compute this capacity: uniform and original capacity. Intrazonal trips are still not included in these two approaches.

\subsection{Approach 1: standard assignment with a uniform capacity on connec- tors}

A uniform capacity on centroid connectors of macro-zone 5 is computed according to equation 5.1. For connectors $5 \rightarrow 1$ and $5 \rightarrow 2$, this capacity is set to $175 \mathrm{veh} / \mathrm{h}$, and to $100 \mathrm{veh} / \mathrm{h}$ for connectors at the opposite direction. Assignment results of this approach are presented in table 5.12. 
In comparison with method 1 and in view of the RD and GEH indicators, setting a finite capacity on centroid connectors is found to improve assignment results and especially to reduce the effect of rerouting. Link $2 \rightarrow 1$ that used to convey zero flow (table 5.11) due to the rerouting problem, is now used by $141 \mathrm{veh} / \mathrm{h}$ (table 5.12). $86 \%$ and $22 \%$ of travel demand between zones 3 to 5 and 5 to 4 , respectively, use link $2 \rightarrow 1$ instead of shortcut routes.

Intrazonal trips are still ignored by this method.

Table 5.12: Simple case: assignment results of method 2.1. Reference flows of connector links 5-1 and 5-2 are Not Available (NA)

\begin{tabular}{ccccccc}
\hline $\begin{array}{c}\text { From } \\
\text { node }\end{array}$ & $\begin{array}{c}\text { To } \\
\text { node }\end{array}$ & $\begin{array}{c}\text { Capacity } \\
\text { (veh/hour) }\end{array}$ & $\begin{array}{c}\text { Flows } \\
\text { (veh/hour) }\end{array}$ & $\begin{array}{c}\text { Reference flows } \\
\text { (veh/hour) }\end{array}$ & $\begin{array}{c}\text { RD } \\
(\%)\end{array}$ & GEH \\
\hline 1 & 2 & 100 & 0 & 50 & -100 & 10 \\
1 & 4 & 100 & 230 & 190 & 22 & 3 \\
1 & 5 & 100 & 100 & NA & NA & NA \\
2 & 1 & 100 & 141 & 297 & -53 & 11 \\
2 & 3 & 100 & 120 & 161 & -26 & 3 \\
2 & 5 & 100 & 100 & NA & NA & NA \\
3 & 2 & 100 & 186 & 158 & 18 & 2 \\
3 & 4 & 100 & 234 & 403 & -23 & 4 \\
4 & 1 & 100 & 14 & 100 & -66 & 5 \\
4 & 3 & 100 & 100 & NA & NA & NA \\
5 & 1 & 175 & 175 & NA & NA & NA \\
5 & 2 & 175 & 175 & & & 0 \\
\hline
\end{tabular}

- Link $1 \rightarrow 4: 230 \approx 100_{1 \rightarrow 4}+52 \% \times 250_{2 \rightarrow 4}$

- $\operatorname{Link} 2 \rightarrow 1: 141 \approx 22 \% \times 250_{2 \rightarrow 4}+86 \% \times 100_{3 \rightarrow 1}$

- Link $2 \rightarrow 3: 120 \approx(1-52 \%) \times 250_{2 \rightarrow 4}$

- Link $3 \rightarrow 2: 186 \approx 100_{3 \rightarrow 2}+86 \% \times 100_{3 \rightarrow 1}$

- Link $3 \rightarrow 4: 234 \approx 100_{3 \rightarrow 4}+(1-86 \%) \times 100_{3 \rightarrow 1}+(1-52 \%) \times 250_{2 \rightarrow 4}$

- Link $4 \rightarrow 1: 14 \approx(1-86 \%) \times 100_{3 \rightarrow 1}$

- $\operatorname{Link} 4 \rightarrow 3: 100 \approx 100_{4 \rightarrow 3}$

- Connector $5 \rightarrow 1: 175 \approx 100_{1 \rightarrow 4}+30 \% \times 250_{2 \rightarrow 4}$

- Connector $5 \rightarrow 2$ : $175 \approx(1-30 \%) \times 250_{2 \rightarrow 4}$

- Connector $1 \rightarrow 5: 100 \approx 86 \% \times 100_{3 \rightarrow 1}+(1-86 \%) \times 100_{3 \rightarrow 1}$

- Connector $2 \rightarrow 5: 100 \approx 100_{3 \rightarrow 2}$

\subsection{Approach 2: standard assignment with original capacity on connectors}


As expected, the use of the original definition of centroids' capacity instead of a uniform distribution improves modeling results (table 5.13).

Table 5.13: Simple case: assignment results of method 2.2. Reference flows of connector links 5-1 and 5-2 are Not Available (NA)

\begin{tabular}{ccccccc}
\hline $\begin{array}{c}\text { From } \\
\text { node }\end{array}$ & $\begin{array}{c}\text { To } \\
\text { node }\end{array}$ & $\begin{array}{c}\text { Capacity } \\
\text { (veh/hour) }\end{array}$ & $\begin{array}{c}\text { Flows } \\
\text { (veh/hour) }\end{array}$ & $\begin{array}{c}\text { Reference flows } \\
\text { (veh/hour) }\end{array}$ & $\begin{array}{c}\text { RD } \\
(\%)\end{array}$ & GEH \\
\hline 1 & 2 & 100 & 0 & 50 & -100 & 10 \\
1 & 4 & 100 & 215 & 190 & 13 & 2 \\
1 & 5 & 100 & 100 & NA & NA & NA \\
2 & 1 & 100 & 205 & 297 & -31 & 6 \\
2 & 3 & 100 & 135 & 161 & -16 & 2 \\
2 & 5 & 100 & 100 & NA & NA & NA \\
3 & 2 & 100 & 191 & 158 & 21 & 3 \\
3 & 4 & 100 & 244 & 403 & -19 & 4 \\
4 & 1 & 100 & 9 & 100 & -79 & 7 \\
4 & 3 & 100 & 100 & NA & NA & NA \\
5 & 1 & 100 & 100 & NA & NA & NA \\
5 & 2 & 250 & 250 & & & 0 \\
\hline
\end{tabular}

- Link $1 \rightarrow 4: 215 \approx 100_{1 \rightarrow 4}+45.6 \% \times 250_{2 \rightarrow 4}$

- Link $2 \rightarrow 1: 205 \approx 45.6 \% \times 250_{2 \rightarrow 4}+91 \% \times 100_{3 \rightarrow 1}$

- Link $2 \rightarrow 3: 135 \approx(1-45.6 \%) \times 250_{2 \rightarrow 4}$

- Link $3 \rightarrow 2: 191 \approx 100_{3 \rightarrow 2}+91 \% \times 100_{3 \rightarrow 1}$

- Link $3 \rightarrow 4: 244 \approx 100_{3 \rightarrow 4}+(1-91 \%) \times 100_{3 \rightarrow 1}+(1-45.6 \%) \times 250_{2 \rightarrow 4}$

- $\operatorname{Link} 4 \rightarrow 1: 9 \approx(1-91 \%) \times 100_{3 \rightarrow 1}$

- Link $4 \rightarrow 3: 100 \approx 100_{4 \rightarrow 3}$

- Connector $5 \rightarrow 1: 100 \approx 100_{1 \rightarrow 4}$

- Connector $5 \rightarrow 2: 250 \approx 250_{2 \rightarrow 4}$

- Connector $1 \rightarrow 5: 100 \approx 91 \% \times 100_{3 \rightarrow 1}+(1-91 \%) \times 100_{3 \rightarrow 1}$

- Connector $2 \rightarrow 5: 100 \approx 100_{3 \rightarrow 2}$

Restraining the capacity of centroid connectors improves modeling results and reduces the bias of rerouting. Therefore, this finite definition is used in all subsequent strategies. As we seek to develop practical methods, the first approach: uniform capacity on centroid connectors is preferred to the second one, since it is more straightforward and needs no detailed information on the distribution of intrazonal data. 


\subsubsection{Method 3: standard assignment with capacity reduction of intrazonal links}

Method 3 takes into account the impact of intrazonal trips on the level of service of intrazonal links. By reducing the capacity of these links, travel times of interzonal trips are likely to increase. However, intrazonal trips are still not assigned to the network and therefore, an underestimation of flows is expected, especially on intrazonal links (table 5.14). The analysis of link flows is therefore limited to the extra-zonal network (links $3 \rightarrow 4$ and $4 \rightarrow 3$ ). For these links, method 3 reproduces more accurately original flows than previous methods with a GEH of less than 2 .

Table 5.14: Simple case: assignment results of method 3. Reference flows of connector links 5-1 and 5-2 are Not Available (NA)

\begin{tabular}{ccccccc}
\hline $\begin{array}{c}\text { From } \\
\text { node }\end{array}$ & $\begin{array}{c}\text { To } \\
\text { node }\end{array}$ & $\begin{array}{c}\text { Capacity } \\
\text { (veh/hour) }\end{array}$ & $\begin{array}{c}\text { Flows } \\
\text { (veh/hour) }\end{array}$ & $\begin{array}{c}\text { Reference flows } \\
\text { (veh/hour) }\end{array}$ & $\begin{array}{c}\text { RD } \\
(\%)\end{array}$ & GEH \\
\hline 1 & 2 & 67 & 0 & 50 & -100 & 10 \\
1 & 4 & 67 & 199 & 190 & 5 & 1 \\
1 & 5 & 100 & 100 & NA & NA & NA \\
2 & 1 & 67 & 98 & 297 & -67 & 14 \\
2 & 3 & 67 & 151 & 161 & -6 & 1 \\
2 & 5 & 100 & 100 & NA & NA & NA \\
3 & 2 & 67 & 174 & 158 & 10 & 1 \\
3 & 4 & 100 & 277 & 403 & -9 & 2 \\
4 & 1 & 67 & 26 & 100 & -39 & 3 \\
4 & 3 & 100 & 100 & NA & NA & NA \\
5 & 1 & 175 & 175 & NA & NA & NA \\
5 & 2 & 175 & 175 & & & 0 \\
\hline
\end{tabular}

- Link $3 \rightarrow 4: 277 \approx 100_{3 \rightarrow 4}+(1-74 \%) \times 100_{3 \rightarrow 1}+(1-39.6 \%) \times 250_{2 \rightarrow 4}$

- Link $4 \rightarrow 3: 100 \approx 100_{4 \rightarrow 3}$

\subsubsection{Method 4: subdivision of zones}

All previous methods do not assign intrazonal demand to the network; method 4 does. This method distributes intrazonal trips between subzones. For this purpose, two distinct distribution approaches are introduced: (1) a simple uniform distribution of total intrazonal trips; (2) the original distribution of intrazonal trips. 


\subsection{Approach 1: subdivision of zones using a uniform distribution of in- trazonal demand}

This approach improves assignment outcomes especially on intrazonal links (table 5.15). As in the case of the original traffic assignment, intrazonal links $1 \rightarrow 2$ and $2 \rightarrow 1$ convey, once again, intrazonal trips. The inclusion of intrazonal demand in traffic assignment is found to counteract the free-flow situation induced by the omission of these trips. Also, the corresponding distribution of network flows reproduces more accurately the original distribution. The maximum GEH indicator is at 6 instead of 24 with the traditional assignment.

Table 5.15: Simple case: assignment results of method 4.1. Reference flows of connector links 5-1 and 5-2 are Not Available (NA)

\begin{tabular}{ccccccc}
\hline $\begin{array}{c}\text { From } \\
\text { node }\end{array}$ & $\begin{array}{c}\text { To } \\
\text { node }\end{array}$ & $\begin{array}{c}\text { Capacity } \\
\text { (veh/hour) }\end{array}$ & $\begin{array}{c}\text { Flows } \\
\text { (veh/hour) }\end{array}$ & $\begin{array}{c}\text { Reference flows } \\
\text { (veh/hour) }\end{array}$ & $\begin{array}{c}\text { RD } \\
(\%)\end{array}$ & GEH \\
\hline 1 & 2 & 100 & 100 & 50 & 99 & 6 \\
1 & 4 & 100 & 213 & 190 & 12 & 2 \\
1 & 5 & 100 & 100 & NA & NA & NA \\
2 & 1 & 100 & 221 & 297 & -25 & 5 \\
2 & 3 & 100 & 137 & 161 & -15 & 2 \\
2 & 5 & 100 & 100 & NA & NA & NA \\
3 & 2 & 100 & 184 & 158 & 17 & 2 \\
3 & 4 & 100 & 253 & 403 & -17 & 3 \\
4 & 1 & 100 & 16 & 100 & -63 & 5 \\
4 & 3 & 100 & 100 & NA & NA & NA \\
5 & 1 & 175 & 175 & NA & NA & NA \\
5 & 2 & 175 & 175 & & & 0 \\
\hline
\end{tabular}

- Link $1 \rightarrow 2: 100 \approx 100_{1 \rightarrow 2}$

- Link $1 \rightarrow 4: 213 \approx 100_{1 \rightarrow 4}+45.2 \% \times 250_{2 \rightarrow 4}$

- Link $2 \rightarrow 1: 221 \approx 100_{2 \rightarrow 1}+15.2 \% \times 250_{2 \rightarrow 4}+84 \% \times 100_{3 \rightarrow 1}$

- $\operatorname{Link} 2 \rightarrow 3: 137 \approx(1-45.2 \%) \times 250_{2 \rightarrow 4}$

- Link $3 \rightarrow 2: 184 \approx 100_{3 \rightarrow 2}+84 \% \times 100_{3 \rightarrow 1}$

- Link $3 \rightarrow 4: 253 \approx 100_{3 \rightarrow 4}+(1-84 \%) \times 100_{3 \rightarrow 1}+(1-45.2 \%) \times 250_{2 \rightarrow 4}$

- Link $4 \rightarrow 1: 16 \approx(1-84 \%) \times 100_{3 \rightarrow 1}$

- Link $4 \rightarrow 3: 100 \approx 100_{4 \rightarrow 3}$

- Connector $5 \rightarrow 1: 175 \approx 100_{1 \rightarrow 4}+30 \% \times 250_{2 \rightarrow 4}$

- Connector $5 \rightarrow 2: 175 \approx(1-30 \%) \times 250_{2 \rightarrow 4}$

- Connector $1 \rightarrow 5$ : $100 \approx 84 \% \times 100_{3 \rightarrow 1}+(1-84 \%) \times 100_{3 \rightarrow 1}$

- Connector $2 \rightarrow 5: 100 \approx 100_{3 \rightarrow 2}$ 


\subsection{Approach 2: subdivision of zones using the original distribution of intrazonal demand}

This approach makes use of intrazonal data to infer the distribution of intrazonal trips and it is only used for comparative purposes only. The use of the original distribution of intrazonal data produces the most accurate results (table 5.16). This approach reproduces exactly reference link flows.

Table 5.16: Simple case: assignment results of method 4.2. Reference flows of connector links 5-1 and 5-2 are Not Available (NA)

\begin{tabular}{ccccccc}
\hline $\begin{array}{c}\text { From } \\
\text { node }\end{array}$ & $\begin{array}{c}\text { To } \\
\text { node }\end{array}$ & $\begin{array}{c}\text { Capacity } \\
\text { (veh/hour) }\end{array}$ & $\begin{array}{c}\text { Flows } \\
\text { (veh/hour) }\end{array}$ & $\begin{array}{c}\text { Reference flows } \\
\text { (veh/hour) }\end{array}$ & $\begin{array}{c}\text { RD } \\
(\%)\end{array}$ & GEH \\
\hline 1 & 2 & 100 & 50 & 50 & 0 & 0 \\
1 & 4 & 100 & 190 & 190 & 0 & 0 \\
1 & 5 & 100 & 99 & NA & NA & NA \\
2 & 1 & 100 & 296 & 297 & 0 & 0 \\
2 & 3 & 100 & 160 & 161 & 0 & 0 \\
2 & 5 & 100 & 101 & NA & 158 & NA \\
3 & 2 & 100 & 158 & 303 & 0 & 0 \\
3 & 4 & 100 & 302 & 43 & -1 & 0 \\
4 & 1 & 100 & 42 & 100 & 0 & 0 \\
4 & 3 & 100 & 100 & NA & NA & NA \\
5 & 1 & 100 & 101 & NA & NA & NA \\
5 & 2 & 250 & 249 & & & 0 \\
\hline
\end{tabular}

- Link $1 \rightarrow 2: 50 \approx 50_{1 \rightarrow 2}$

- Link $1 \rightarrow 4: 190 \approx 100_{1 \rightarrow 4}+36 \% \times 250_{2 \rightarrow 4}$

- Link $2 \rightarrow 1: 298 \approx 150_{2 \rightarrow 1}+36 \% \times 250_{2 \rightarrow 4}+58 \% \times 100_{3 \rightarrow 1}$

- Link $2 \rightarrow 3: 160 \approx(1-36 \%) \times 250_{2 \rightarrow 4}$

- Link $3 \rightarrow 2: 158 \approx 100_{3 \rightarrow 2}+58 \% \times 100_{3 \rightarrow 1}$

- Link $3 \rightarrow 4: 302 \approx 100_{3 \rightarrow 4}+(1-58 \%) \times 100_{3 \rightarrow 1}+(1-36 \%) \times 250_{2 \rightarrow 4}$

- Link $4 \rightarrow 1: 42 \approx(1-58 \%) \times 100_{3 \rightarrow 1}$

- Link $4 \rightarrow 3: 100 \approx 100_{4 \rightarrow 3}$

- Connector $5 \rightarrow 1: 100 \approx 100_{1 \rightarrow 4}$

- Connector $5 \rightarrow 2: 250 \approx 250_{2 \rightarrow 4}$

- Connector $1 \rightarrow 5$ : $100 \approx 58 \% \times 100_{3 \rightarrow 1}+(1-58 \%) \times 100_{3 \rightarrow 1}$

- Connector $2 \rightarrow 5: 100 \approx 100_{3 \rightarrow 2}$ 


\subsubsection{Discussion}

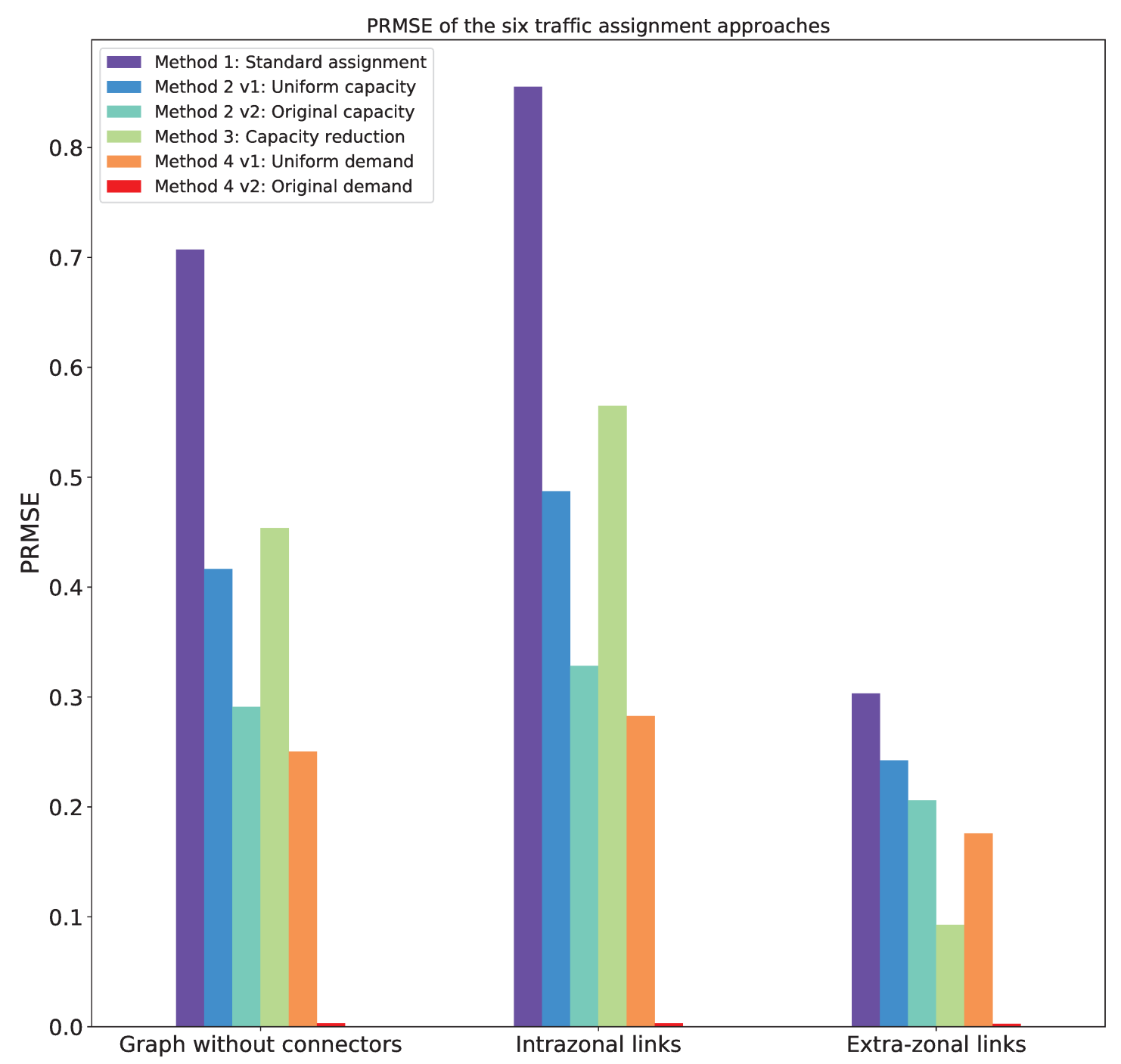

Figure 5.7: Simple case: PRMSE of link flows of the 6 assignment strategies. Color bars are displayed in the order of the legend. The PRMSE of Method 4.2 is nearly 0

The PRMSE is computed to assess the contribution of each assignment method. According to this goodness-of-fit indicator and to the analysis above, method 4 is found to produce the most accurate assignment results (figure 5.7). Original link flows are accurately reproduced by both approaches of this method. Furthermore, the standard traffic assignment, i.e. method 1, has the highest error rates, especially on intrazonal links. As one might expect, the original distribution of intrazonal demand and the original definition of connectors produce better results than uniform distributions. Nevertheless, the current case study is too simple to draw realistic and generalizable conclusions. To this end, a slightly more complex case studies is used in the next section.

The use of a finite capacity on centroid connectors is also found to improve modeling results. The 
extent of the rerouting of flows from congested to uncongested routes induced by connectors is reduced. Nevertheless, setting a capacity on a connector does not guarantee after aggregation that this connector will be used by the same trips as in the original case study. Other trips originating from other centroids might as well use this connector to produce a new network equilibrium.

To illustrate this case, we introduce a variant of the previous simple case study. This time, zone 1 sends 100 car trips to zone 3 , and zone 2 sends 200 car trips to zone 4 (table 5.17). We also assume that links $1 \rightarrow 2$ and $2 \rightarrow 1$ are congested and cannot be used by additional car trips. In this case, before aggregation, demand from zone 1 to 3 should only use links $1 \rightarrow 4 \rightarrow 3$ and demand from zones 2 to 4 should use links $2 \rightarrow 3 \rightarrow 4$ (table 5.18). After aggregation and after setting a finite capacity on centroid connectors, a new macro-zone 5 and two connectors are introduced: connector $5 \rightarrow 1$ with a capacity of $100 \mathrm{veh} / \mathrm{h}$ and connector $5 \rightarrow 2$ with a capacity of $200 \mathrm{veh} / \mathrm{h}$. In this case, the distribution of flows on links $3 \rightarrow 4$ and $4 \rightarrow 3$ is different from the original one despite setting a finite capacity on centroid connectors (table 5.18).

Table 5.17: Original travel demand of the rerouting example

\begin{tabular}{ccc}
\hline Origin & Destination & Demand \\
\hline 1 & 3 & 100 \\
2 & 4 & 200 \\
\hline
\end{tabular}

Table 5.18: Assignment results of the rerouting example

\begin{tabular}{ccccc}
\hline From node & To node & Capacity (veh/hour) & Flows (veh/hour) & Reference flows (veh/hour) \\
\hline 1 & 2 & 100 & 0 & 0 \\
1 & 4 & 100 & 100 & 100 \\
1 & 5 & 0 & 0 & 0 \\
2 & 1 & 100 & 0 & 0 \\
2 & 3 & 100 & 200 & 200 \\
2 & 5 & 0 & 0 & 0 \\
3 & 2 & 100 & 0 & 0 \\
3 & 4 & 100 & 118 & 200 \\
4 & 1 & 100 & 0 & 0 \\
4 & 3 & 100 & 18 & 100 \\
5 & 1 & 100 & 100 & 100 \\
5 & 2 & 200 & 200 & 200 \\
\hline
\end{tabular}

This example clearly demonstrates that it is not always possible to reproduce original link flows even after the inclusion of intrazonal trips or the use of a finite capacity on connectors. The rerouting 
problem will often be at play when aggregation is performed. Current assignment strategies are therefore intended to reduce, as far as possible, the extent of this problem.

\subsubsection{Sioux Falls case study}

Five spatial designs are produced to confirm or to reject findings from the simple case study. In the current section, we examine in detail assignment results of zoning 2 where zones $1,3,4,11,12,13$, 14, 23, and 24 are merged to produce a new macro-zone with an intrazonal rate of $12 \%$ (table 5.7). The rest zonings are discussed in Appendix A.

\subsubsection{Original case study}

The original zoning and travel demand of the Sioux Falls case study are assigned to the network to produce reference link flows (figure 5.8). 


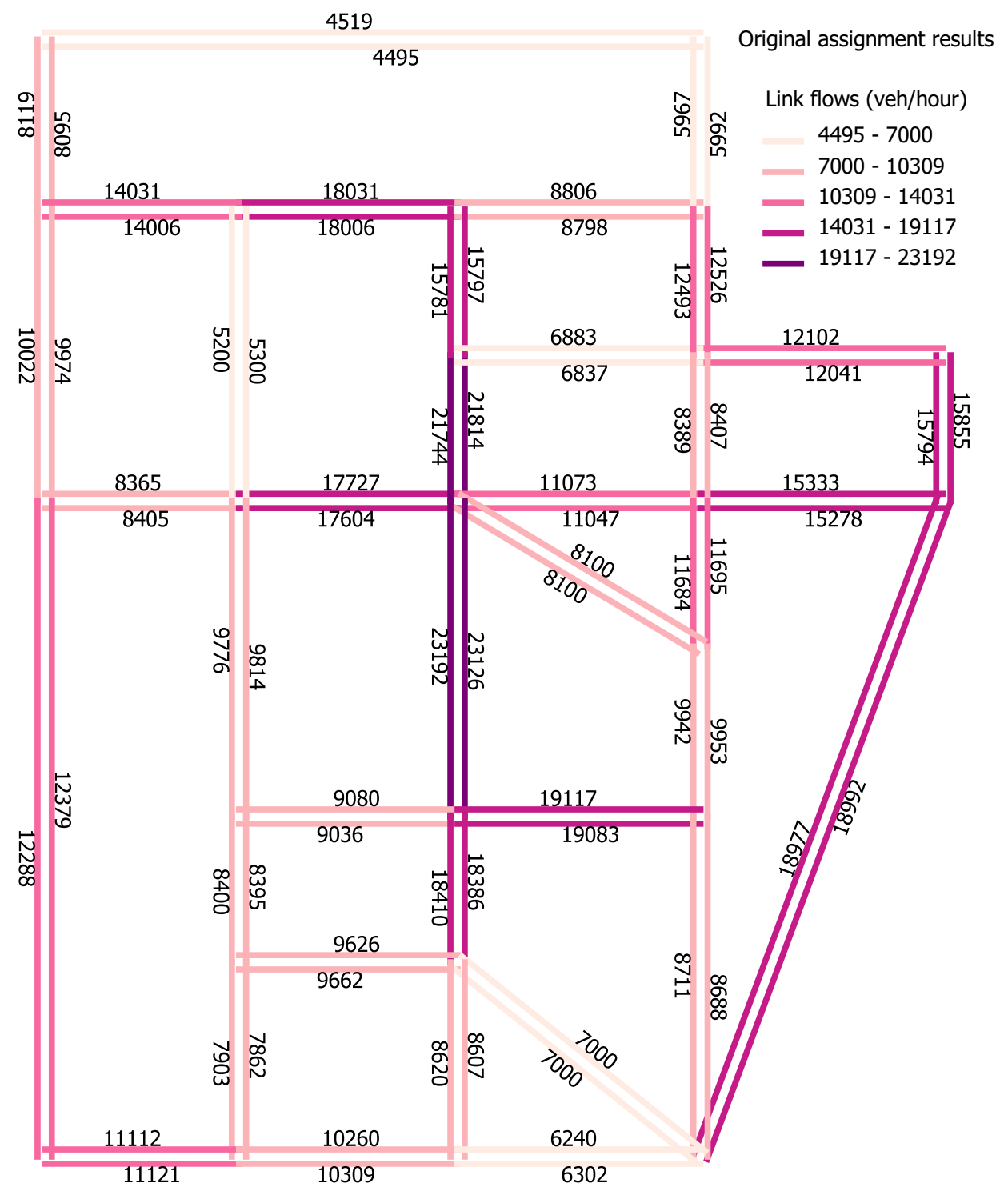

Figure 5.8: Sioux Falls: original link flows. Link flows may differ from those known in the literature due to differences in convergence criteria

\subsubsection{Method 1: standard assignment method with infinite capacity on con- nectors}

Results from the conventional assignment show that the intrazonal network is the most impacted by aggregation. In this regard, conventional traffic assignment underestimates link flows on these links. This underestimation is due to two factors: the omission of intrazonal trips and the rerouting 
of flows (figure 5.9). 16\% of network links have an Absolute Relative Difference (ARD) indicator greater than $50 \%$. $62 \%$ of network links have an RD indicator between $-20 \%$ and $20 \%$. More than $61 \%$ of network links have a GEH indicator greater than 10.

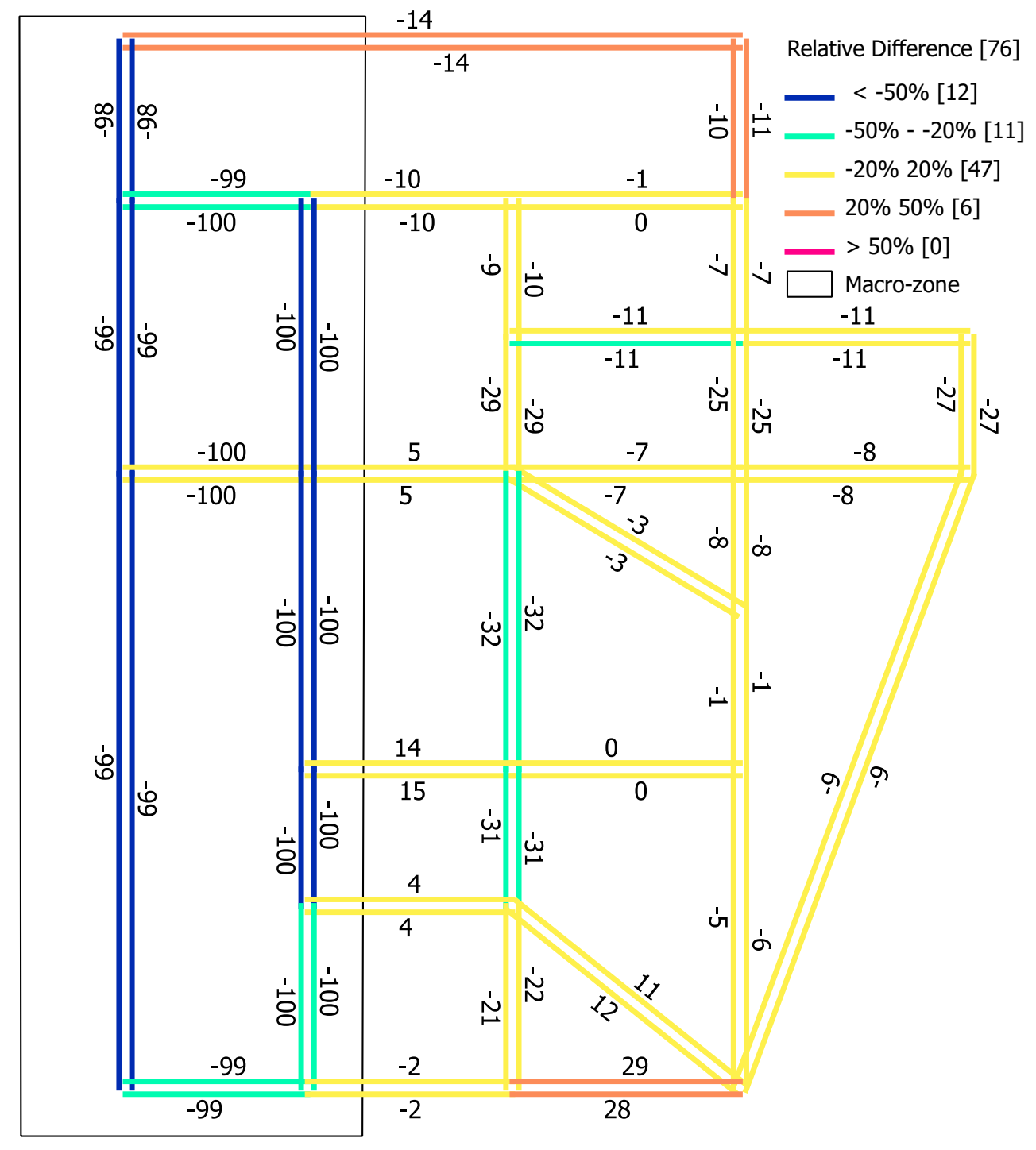

Figure 5.9: Sioux Falls: the relative difference (RD) of link flows produced by method 1.

To correct for modeling errors induced by the traditional assignment and the spatial aggregation bias, 5 new assignment strategies are applied to this case study. 


\subsubsection{Method 2: standard assignment method with a finite capacity on con- nectors}

\subsection{Approach 1: standard assignment with a uniform capacity on connec- tors}

Setting a finite and uniform capacity on centroid connectors improves the accuracy of assignment results. The underestimation of intrazonal link flows is less pronounced than with the previous method but results are still prone to error. $61 \%$ of all network links have a moderate error between -20\% and 20\% and 12 links only are still affected by an Absolute Relative Difference (ARD) greater than $50 \%$ (figure 5.10). The GEH indicator is still high but it is less high than that of the standard method. 


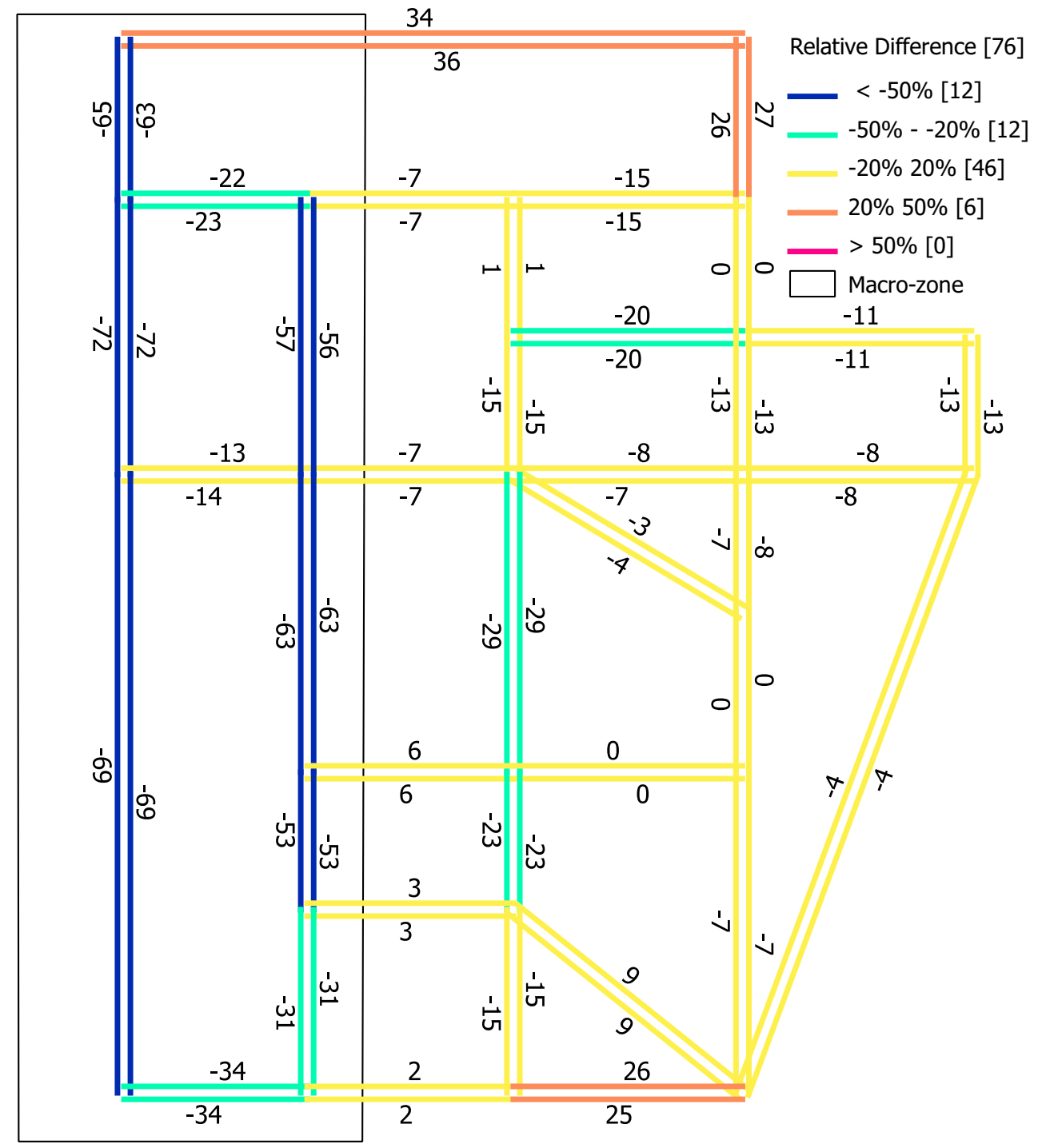

Figure 5.10: Sioux Falls: the relative difference (RD) of link flows produced by method 2.1

\subsection{Approach 2: standard assignment with original capacity on connectors}

Surprisingly, the use of the original capacity on connectors has a limited contribution to modeling results when compared to the uniform approach. Assignment outcomes pertaining to this approach are similar to those in figure 5.10 and tables 5.19, 5.20 (see Appendix A). 


\subsubsection{Method 3: standard assignment with capacity reduction of intrazonal links}

Results from method 3 are similar to those of method 2. No clear contribution can be drawn from this method especially on extra-zonal links (see Appendix A).

\subsubsection{Method 4: subdivision of zones}

\subsection{Approach 1: subdivision of zones using a uniform distribution of in- trazonal demand}

By including intrazonal demand in the assignment procedure, the quality of assignment results improves significantly. All network links have a Relative Difference (RD) error between -28\% and 49\%. $75 \%$ of all network links have an RD indicator between $-20 \%$ and $20 \%$. $50 \%$ of network links have a GEH indicator less than 10 (figure 5.11). 


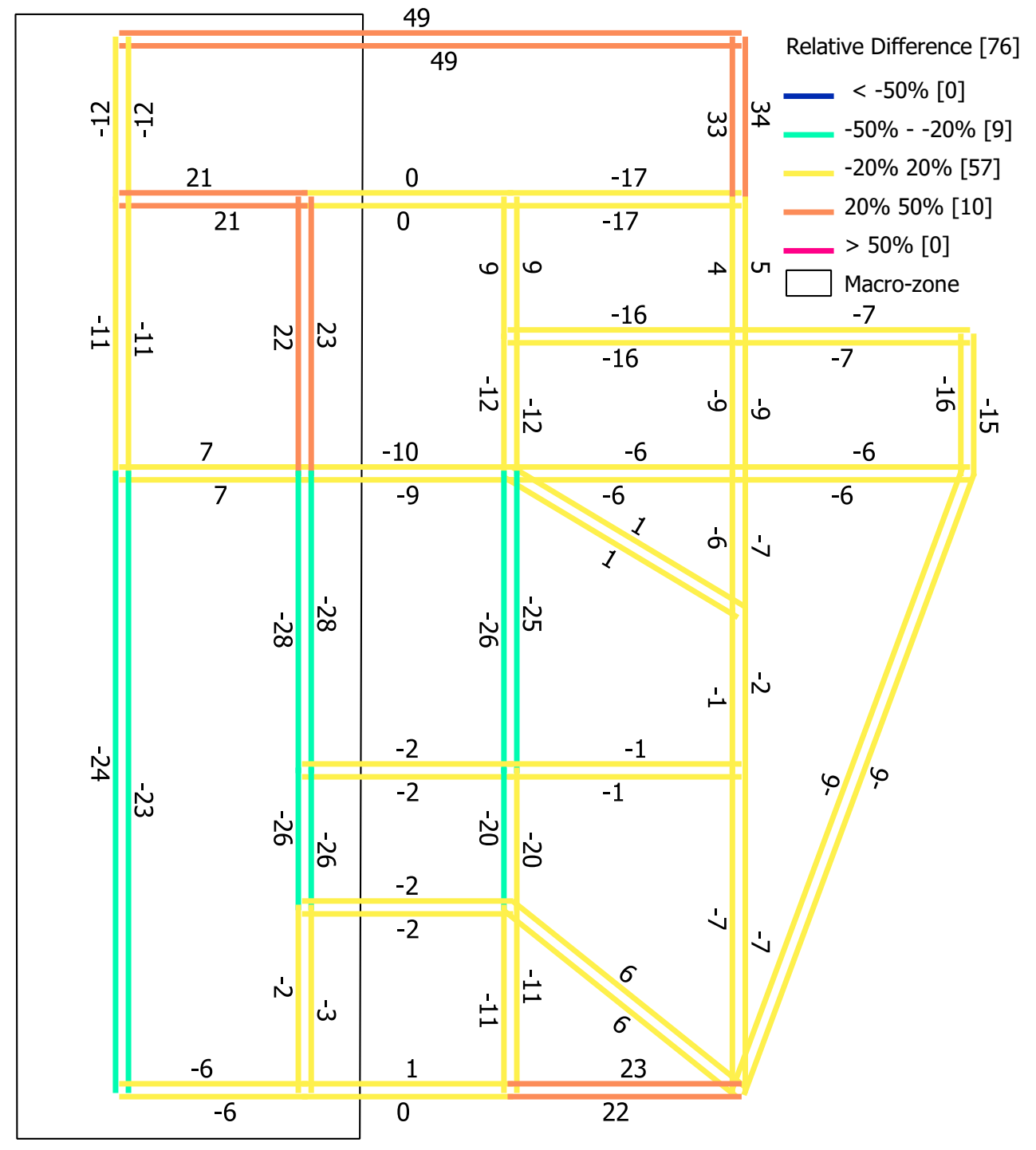

Figure 5.11: Sioux Falls: the relative difference (RD) of link flows produced by method 4.1

\subsection{Approach 2: subdivision of zones using the original distribution of intrazonal demand}

As expected, the use of the original distribution of intrazonal demand is found to improve assignment outcomes. Both intrazonal and extra-zonal links reproduce more accurately reference link flows from the original case study. $97 \%$ of network links have a relative difference $(\mathrm{RD})$ indicator between $-20 \%$ and $20 \%$. $59 \%$ of network links have a satisfactory GEH indicator less than 5 . This proportion is of $16 \%$ with the standard assignment (figure 5.12). 


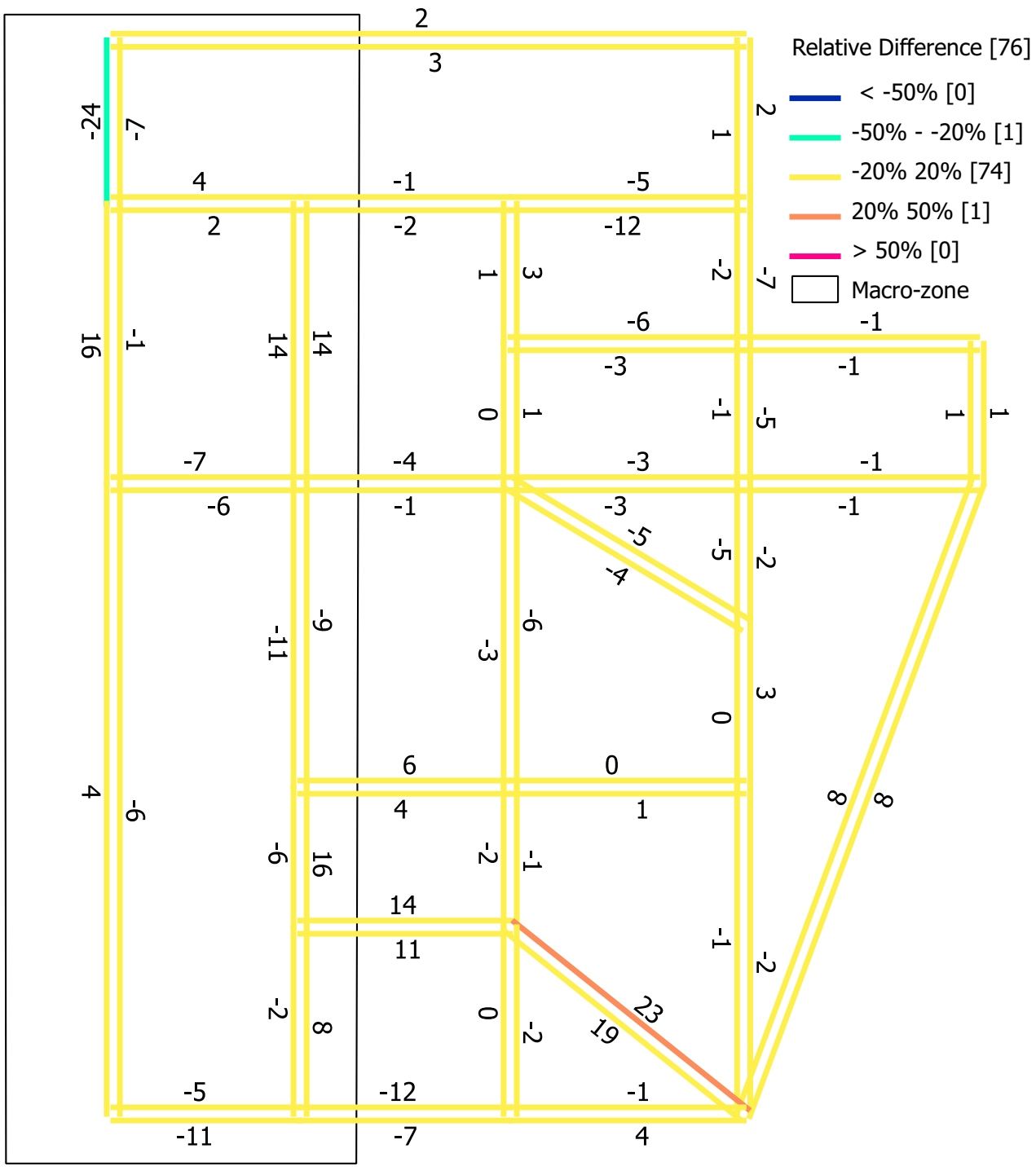

Figure 5.12: Sioux Falls: the relative difference (RD) of link flows produced by method 4.2 


\subsubsection{Discussion}

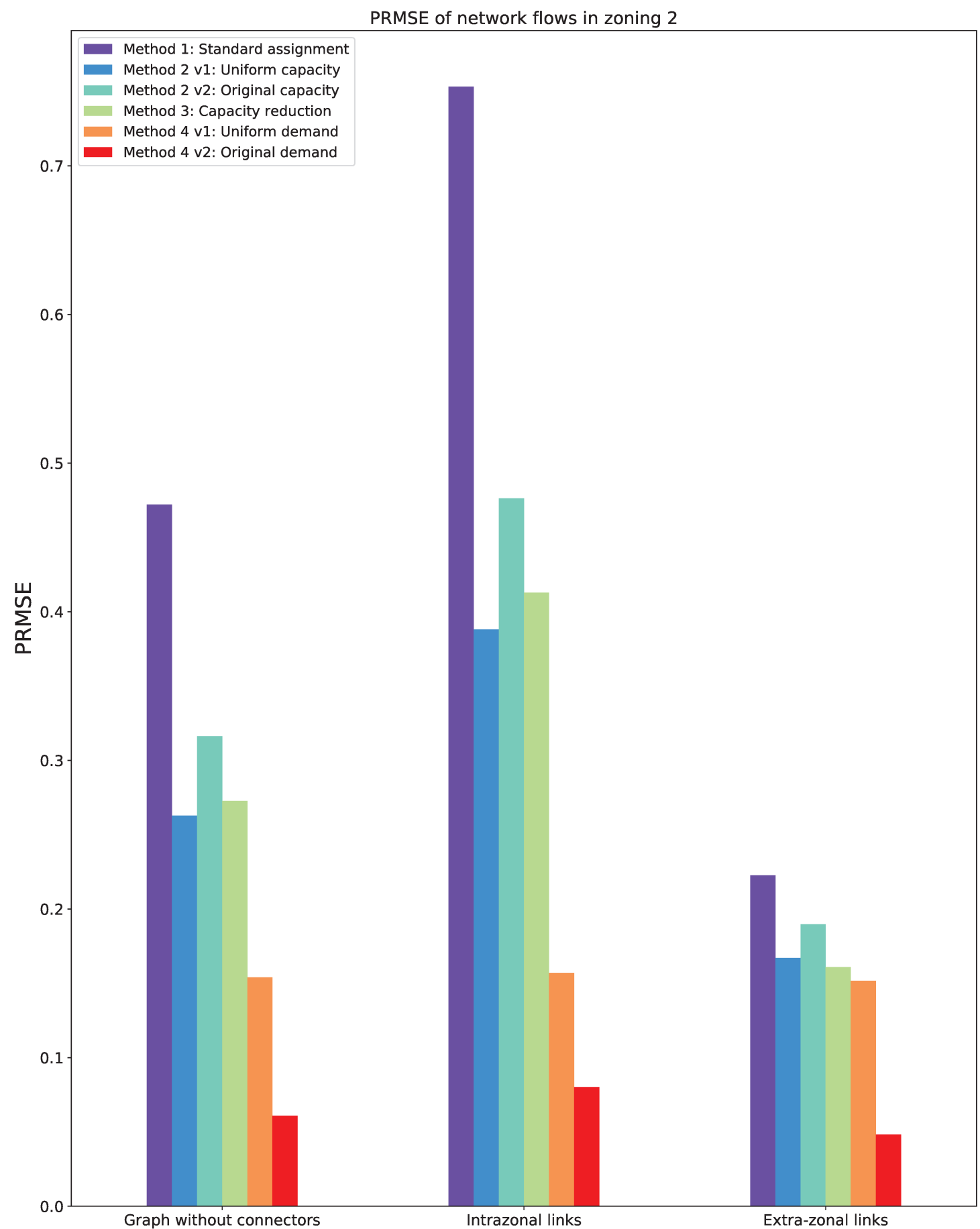

Figure 5.13: Sioux Falls: the PRMSE of link flows of the six assignment methods. Color bars are displayed in the order of the legend 
Table 5.19: Sioux Falls: descriptive statistics of the distribution of the Absolute Relative Difference (ARD) on network links

\begin{tabular}{ccccccc}
\hline Statistics & Method 1 & Method 2.1 & Method 2.2 & Method 3 & Method 4.1 & Method 4.2 \\
\hline count & 76 & 76 & 76 & 76 & 76 & 76 \\
mean & 35.30 & 20.84 & 25.78 & 21.99 & 12.23 & 5.27 \\
std & 39.48 & 20.78 & 25.11 & 22.92 & 10.72 & 5.25 \\
min & 0.242 & 0.017 & 0.34 & 0.04 & 0.11 & 0.16 \\
$25 \%$ & 7.22 & 6.64 & 6.93 & 6.20 & 5.63 & 1.34 \\
$50 \%$ & 13.05 & 13.29 & 16.88 & 12.92 & 9.213 & 3.43 \\
$75 \%$ & 97.97 & 29.11 & 30.72 & 30.90 & 20.03 & 6.725 \\
max & 99.99 & 72.41 & 87.62 & 73.88 & 49.27 & 24.23 \\
\hline
\end{tabular}

Table 5.20: Sioux Falls: descriptive statistics of the distribution of the GEH on network links

\begin{tabular}{ccccccc}
\hline Statistics & Method 1 & Method 2.1 & Method 2.2 & Method 3 & Method 4.1 & Method 4.2 \\
\hline count & 76 & 76 & 76 & 76 & 76 & 76 \\
mean & 46.46 & 23.08 & 29.66 & 24.38 & 12.46 & 5.17 \\
std & 55.80 & 24.88 & 30.38 & 27.06 & 10.19 & 4.7 \\
min & 0.23 & 0.02 & 0.47 & 0.06 & 0.15 & 0.16 \\
$25 \%$ & 8.120 & 6.345 & 7.43 & 6.416 & 5.25 & 1.59 \\
$50 \%$ & 12.95 & 13.97 & 15.77 & 14.73 & 9.58 & 3.72 \\
$75 \%$ & 102.2 & 30.07 & 35.97 & 31.16 & 18.31 & 6.97 \\
max & 166.4 & 94.71 & 108.1 & 97.36 & 41.68 & 23.29 \\
\hline
\end{tabular}

Findings from this case study confirm that the traditional assignment method is the less accurate assignment strategy (figure 5.13 and tables 5.19, 5.20). The traditional assignment produces a systematic underestimation of link flows and higher error rates. When compared to other assignment strategies, this method fails to reproduce original link flows, especially on intrazonal links.

The use of a finite capacity on connectors without assigning intrazonal trips contributes to a slight degree to the accuracy of modeling outcomes. This method reduces to some extent the impact of the rerouting problem. Remarkably, in this case study, the first approach that uses a uniform distribution of capacity proves to be more accurate than the original distribution. In other case studies presented in Appendix A, the adverse outcome is found.

In comparison with method 2 , the capacity reduction method proves to have no specific contribution (figure 5.13, tables 5.19, 5.20). In fact, the contribution of method 3 over the standard method is totally due to the constrained definition of the capacity of connectors. Method 3 builds on method 
2 by adding a capacity reduction factor on intrazonal links (see section Methods). In this regard, methods 2 and 3 produce similar results. These findings are consistent with the outcomes of other spatial designs (see Appendix A). For this reason, this assignment strategy is discarded from analysis in the next case study of Lyon.

The results above also suggest that both approaches of method 4 introduce an outstanding improvement of assignment outcomes. By including intrazonal trips in traffic assignment, modeling errors decrease and model accuracy increases especially in the case of method 4.2 (figure 5.13, table 5.19, table 5.20). The PRMSE indicator is of about $15 \%$ in the first approach and of $5 \%$ in the second. These figures are to be compared with the standard assignment that produces a PRMSE indicator of 47\%. The same applies to the GEH indicator that has the lowest values in method 4.2 (table 5.20). Method 4.1 is less accurate than method 4.2; still, the former approach has a significant contribution over the rest of methods: 1,2 , and 3, and overall, it has the advantage of being practical and needs no detailed intrazonal data.

It is noteworthy that despite the use of the original distribution of intrazonal demand in method 4.2, this method is still prone to bias (figure 5.13). Method 4.2 has the same total travel demand as the original trip table and the same distribution of intrazonal trips. The capacity of connectors is also deduced from their original definition. In this case, assignment errors are solely due to the rerouting problem: aggregated travel demand uses different network routes than that used in the original case study. Using a constrained definition of the capacity of connectors has been proven to partly reduce the extent of this problem.

Method 4 has proven to produce the most accurate results. A detailed investigation of this method is carried out in the case study of Lyon.

\subsubsection{Lyon case study}

3 of the 6 previous assignment methods are further examined in the case study of Lyon (table 5.21).

Table 5.21: Assignment methods of the Lyon case study

\begin{tabular}{llll}
\hline Method & Graph & Connectors & Demand \\
\hline 1 & Original & Finite \& uniform capacity & No intrazonal demand \\
4.1 & Original & Finite \& uniform capacity & Uniform distribution \\
4.2 & Original & Finite \& variable capacity & Original distribution \\
\hline
\end{tabular}

For the purpose of getting robust and generalizable findings, a simulation framework based on stochastic modeling is used. In contrast with previous case studies where the impact of aggregation is appraised using only one spatial design at once, the case study of Lyon uses instead a stochastic 
approach with 700 different zonings to derive robust conclusions (see Lyon case study).

\subsubsection{Original case study}

The original case study of Lyon combines a detailed zoning of 777 zones and a detailed description of the network links (figure 5.4, table 5.8). Assignment outcomes of the original case study are considered as reference flows. The contribution of methods 4.1 and 4.2 is appraised against these flows. Centroid connectors are defined using a finite capacity as described in method 2.

The statistical distribution of reference link flows described in table 5.22:

Table 5.22: Descriptive statistics of reference link flows according to road category. Average link flows and deviation are computed using the length of links as a weighting factor

\begin{tabular}{ccccccccc}
\hline $\begin{array}{c}\text { Road } \\
\text { Class }\end{array}$ & $\begin{array}{c}\text { Average Reference flows } \\
\text { (veh/hour) }\end{array}$ & $\begin{array}{c}\text { Std } \\
\text { (veh/hour) }\end{array}$ & Min & $25 \%$ & Median & $75 \%$ & $99 \%$ & Max \\
\hline 1 & 1,653 & 690 & 0 & 929 & 1,635 & 2,187 & 4,354 & 4,609 \\
2 & 1,199 & 1,313 & 0 & 398 & 772 & 1,436 & 5,187 & 6,672 \\
3 & 430 & 450 & 0 & 94 & 291 & 600 & 2,350 & 4,860 \\
4 & 181 & 239 & 0 & 19 & 89 & 254 & 1,085 & 2,819 \\
5 & 66 & 144 & 0 & 0 & 0 & 54 & 724 & 1,317 \\
\hline
\end{tabular}

\subsubsection{Contribution of assignment strategies}

The traditional assignment method is found to produce biased assignment outcomes. This bias is due to the omission of intrazonal demand and to the rerouting problem introduced by connectors. The new assignment strategies intend to counteract, as far as possible, this bias by re-integrating intrazonal trips into assignment models and by reducing the effect of rerouting. The contribution of these strategies is appraised using various assignment outcomes (eq. 5.7): average link flows $(\bar{Q})$, average link flows by road category $\left(\bar{Q}_{c}\right)$, total Vehicle-Kilometers of Travel $(V K T)$, total VKT by road category $\left(V K T_{c}\right)$.

$$
\begin{aligned}
& V K T_{C}=\sum_{i \in C} l_{i} \times q_{i} \\
& V K T=\sum_{j \in C} V K T_{j} \\
& \overline{Q_{C}}=\frac{V K T_{C}}{\sum_{j \in C} l_{j}} \\
& \bar{Q}=\sum_{C \in H} \overline{Q_{C}}
\end{aligned}
$$


Where $l_{i}$ is the length of link $i . C$ is a road hierarchy as described in table 5.8. $H$ is the set of all road hierarchies.

\subsection{Method 1: standard assignment method with infinite capacity on con- nectors}

As demonstrated in chapter 4, the traditional traffic assignment is subject to spatial aggregation errors. This bias has already been demonstrated in the case study of Lyon using data from 2006. Findings from this section confirm this conclusion in a more up-to-date case study of 2015 .

Network flows are correlated with intrazonal rates (figure 5.14). This correlation is negative and equals -0.987. High intrazonal trip rates underestimate flows on the network and produce erroneous travel times. This finding differs between road categories.

When the analysis is conducted on each road hierarchy, the correlation pattern above persists. As we go further in road hierarchy (from highway roads to local streets), the effect of aggregation becomes more pronounced (sub-figures $b, c, d, e$, and $f$ in figure 5.14). As one might expect, local streets are the most impacted links. This is mainly for two reasons: the omission of intrazonal trips and the rerouting of flows. Intrazonal trips often use the lowest road hierarchies: local streets and collectors and barely high hierarchies: highway roads or arterial roads. Moreover, connectors are often attached to low hierarchy links, and therefore local streets are more likely to be impacted by the rerouting problem induced by these connectors than highway roads. By ignoring these two detrimental effects, the traditional traffic assignment produces biased results, especially on low hierarchy roads (figures $e$ and $f$ in figure 5.14). 

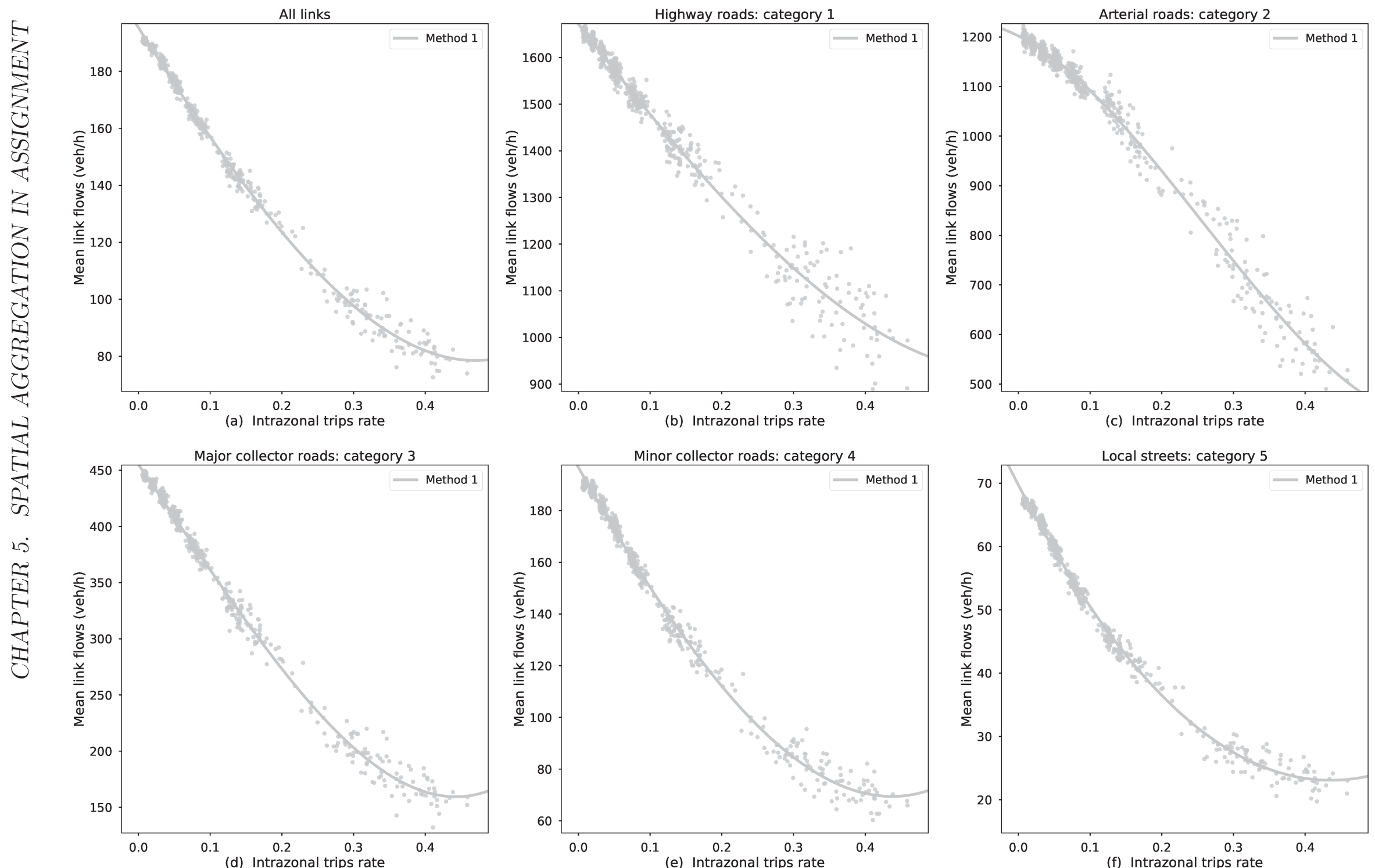

Figure 5.14: Lyon: correlation between link flows and intrazonal trips of method 1 according to road hierarchy. The X-axis represents the rate of intrazonal trips and Y-axis depicts average link flows. The mean is computed using the length of links as a weight 


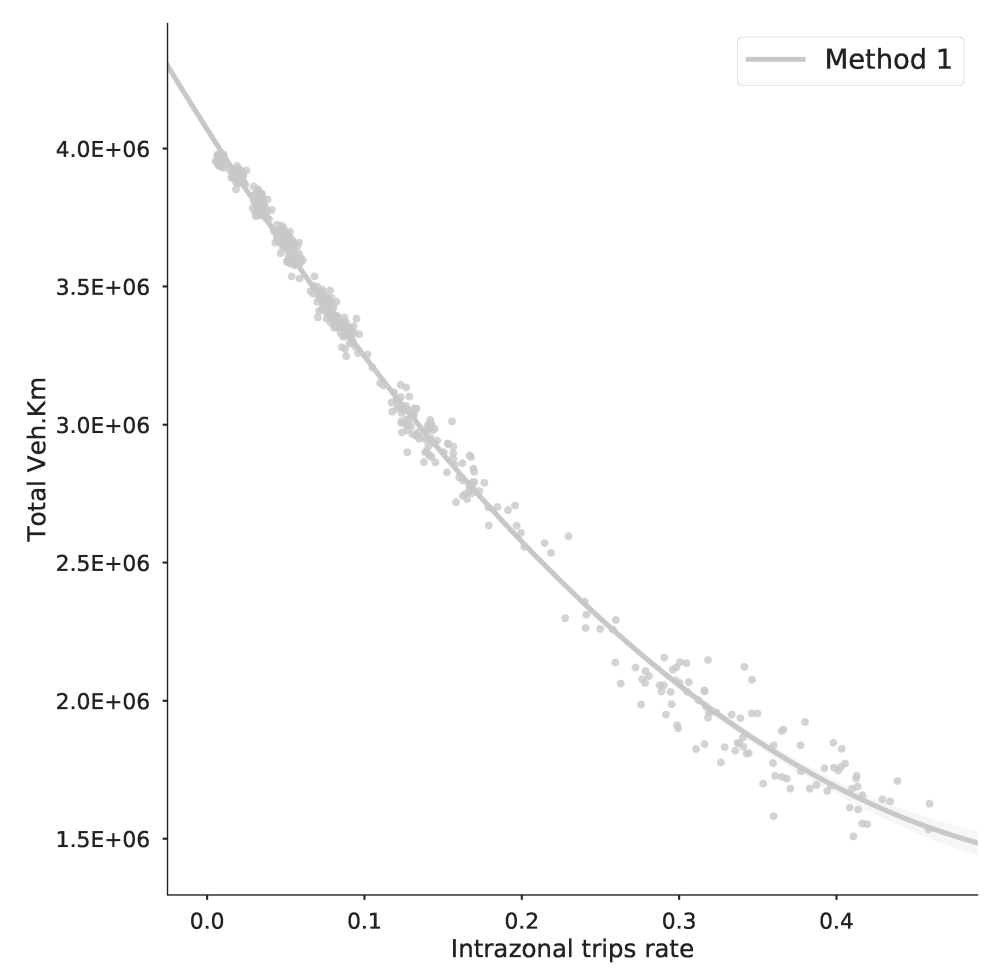

Figure 5.15: Lyon: correlation between Vehicle-Kilometers of Travel (VKT) and intrazonal trips on the overall network of assignment method 1. The X-axis represents the rate of intrazonal trips and Y-axis depicts total vehicle-kilometers of travel on the network

Figure 5.15 depicts the correlation between the total vehicle-kilometers of travel (VKT) and intrazonal trips rate. This correlation is negative, i.e. The VKT indicator drops when aggregation is performed. The extent of this decline is remarkably significant: VKT drops from nearly $4 \times 10^{6}$ to $1.5 \times 10^{6}$ vehicle-kilometers. This drop is a direct consequence of using an assignment method that is subject to spatial aggregation errors.

\subsection{Method 4.1: subdivision around zones and uniform distribution of intrazonal demand}

Results drawn from method 4.1 outline two major findings:

- A general improvement of assignment outcomes.

- A U-shape pattern.

Assignment results from method 4.1 are less subject to the free-flow bias as noticed in the standard traffic assignment (figure 5.16). The drop in main assignment outcomes is still persistent but it is more controlled for. This is especially the case of simulations with a high intrazonal trips rate (greater than $20 \%$ for example). First results confirm previous findings on the fact that this method 

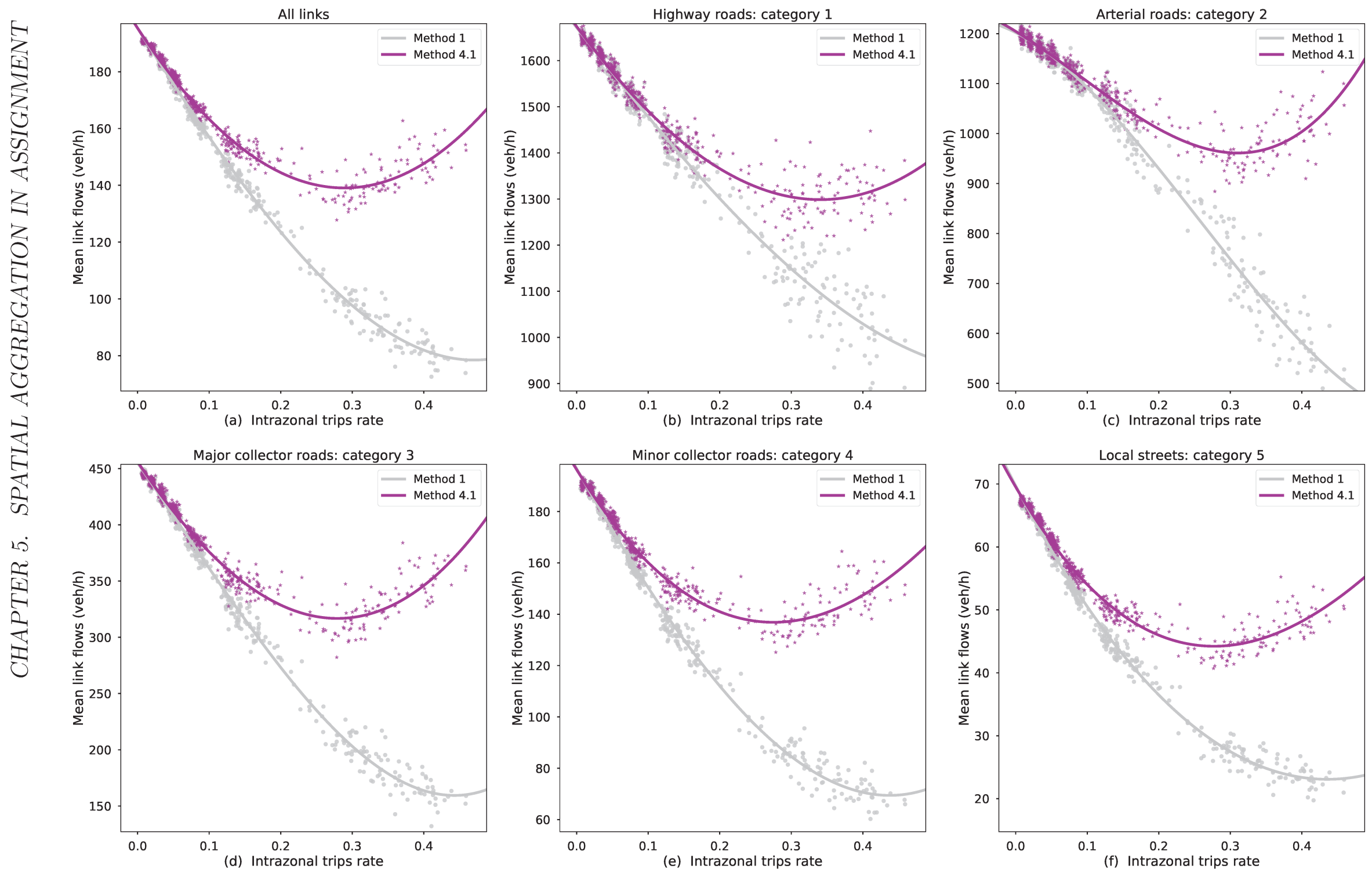

Figure 5.16: Lyon: correlation between link flows and intrazonal trips of methods 1 and 4.1 according to road hierarchy. The

ำ $\mathrm{X}$-axis represents the rate of intrazonal trips and Y-axis depicts average link flows. The mean is computed using the length of links as a weight 
outperforms the standard one in reproducing original link flows. This contribution differs between road hierarchies.

The contribution brought by method 4.1 is clearly visible on low hierarchy roads, i.e. local streets and minor collectors. For simulations with 200 and 100 zones, average link flows on road categories 4 and 5 has increased by more than $70 \%$ after the inclusion of intrazonal demand in the assignment. In simulations with a rate of intrazonal trips lower than $10 \%$, the contribution of method 4.1 over the traditional assignment is less clear.

The decrease in average traffic flows and VKT is less sharp than with the traditional assignment, but it is still significant. For all five road hierarchies, this findings holds (sub-figures $b, c, d, e$, and $f$ in figure 5.16). The new assignment strategy improves assignment outcomes and reproduces more accurately original link flows for all network links, and especially for low hierarchy links.

Despite the improvement of assignment outcomes, one might still notice a decrease in average link flows and total VKT. In this case, the decrease is not due to any omission of intrazonal trips: total travel demand is constant and equals the original one. Furthermore, the decrease in average link flows and total vehicle-kilometers is characterized by an unexpected parabolic pattern. This pattern is discussed in detail in the Discussion section.

\subsection{Method 4.2: subdivision around zones and original distribution of intrazonal demand}

Method 4.2 is similar to method 4.1 in all respects apart from the distribution of intrazonal demand between subzones and the definition of the capacity of centroid connectors. In method 4.2 intrazonal demand and connectors are defined using detailed data from the original case study.

As expected, method 4.2 is less subject to the fictitious free-flow bias than the standard assignment (figure 5.17). Average link flows produced by this method depict a U-shape when the rate of intrazonal trips increases. In contrast with previous findings, method 4.1 seems to outperform method 4.2 in reproducing average original link flows on all road hierarchies (sub-figures $b, c, d$, $e$, and $f$ in figure 5.17). The same conclusion holds for total Vehicle-Kilometers of Travel (VKT) (figure 5.18). This finding does not imply necessarily that method 4.1 outperforms method 4.2 in reproducing original link flows which is the actual definition of contribution. To this end, a detailed examination of the contribution of each method is undertaken in the next section. An explanation of this unexpected outperformance is also given in the Discussion section. 

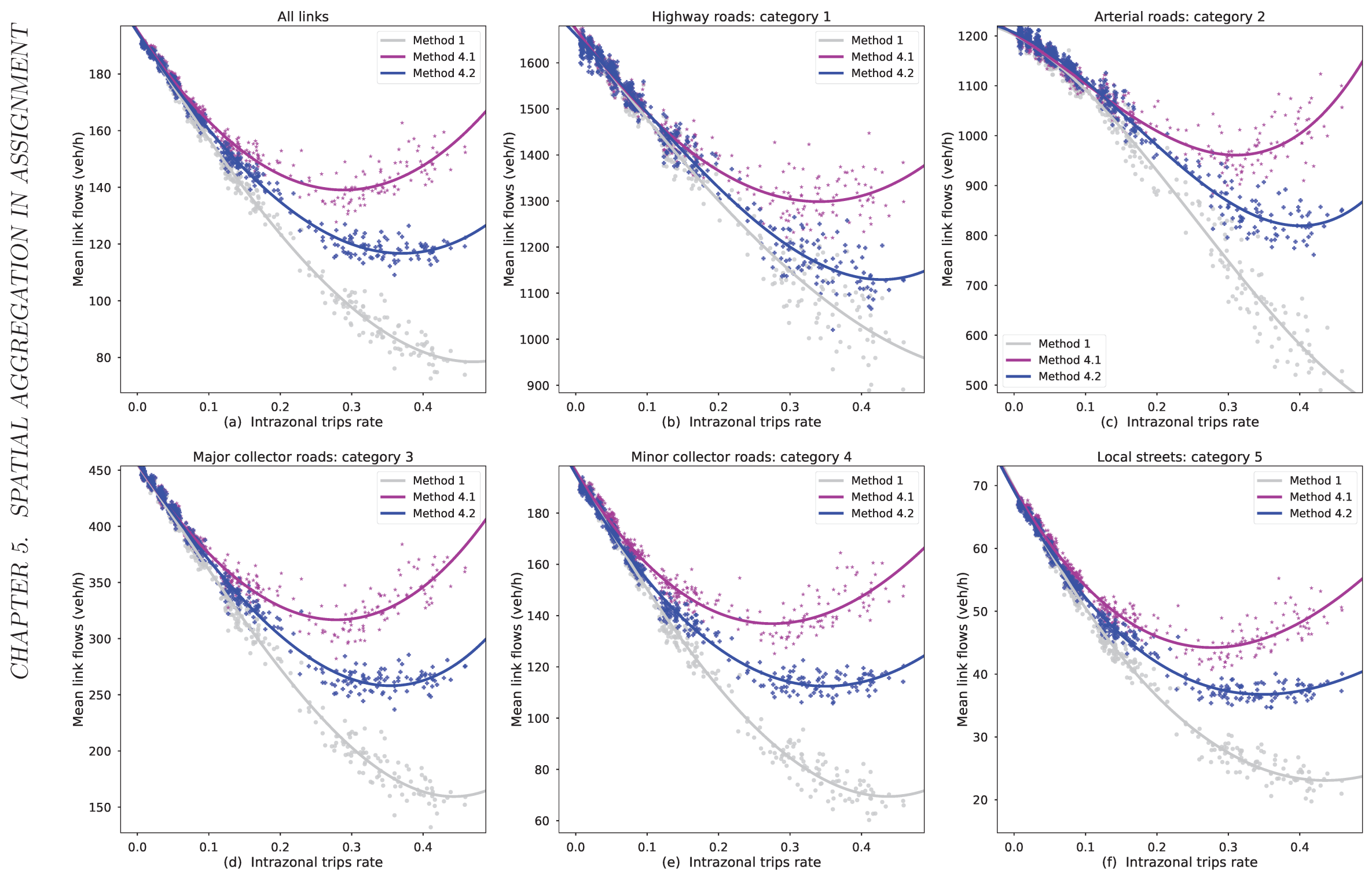

Figure 5.17: Lyon: Correlation between link flows and intrazonal trips of methods 1, 4.1, and 4.2 according to road hierarchy. 


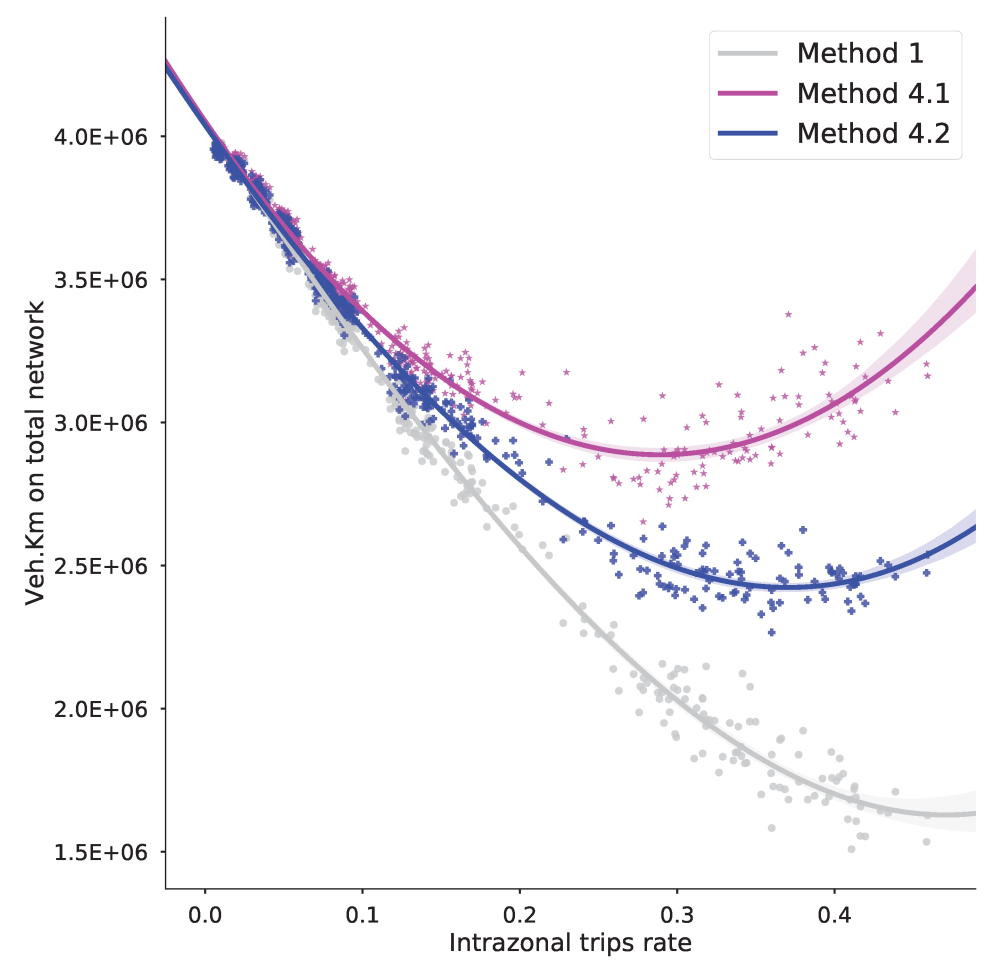

Figure 5.18: Lyon: correlation between Vehicle-Kilometers of Travel (VKT) and intrazonal trips of methods $1,4.1$, and 4.2. The $\mathrm{X}$-axis represents the rate of intrazonal trips and Y-axis depicts vehicle-kilometers of travel on the network

The next section investigates the accuracy of modeling outcomes.

\subsubsection{Assignment methods: accuracy}

The contribution of a method is computed with reference to the original case study. The accuracy of a method is defined as its capacity to reproduce original link flows. To assess this capacity, the PRMSE and GEH measures are used. These measures are deemed to be relevant in comparing data of the same magnitude. For this reason, accuracy analysis is conducted separately on each road category.

For all assignment strategies, the accuracy of modeling results diminishes as aggregation goes on (figures 5.19 and 5.20). The traditional assignment method has the highest PRMSE values on all road categories. This means that the standard method is less accurate than the remaining assignment strategies. Methods 4.1 and 4.2 have the lowest PRMSE values. With comparison to the standard strategy, methods 4.1 and 4.2 are found to minimize the aggregation bias on all road hierarchies and especially on low levels (sub-figures $a, b$, and $c$ in 5.19 and 5.20). Results also suggest that method 4.1 outperforms method 4.2 in reproducing link flows on high hierarchy roads 
(figure 5.19). On the contrary, method 4.2 outperforms method 4.1 on low road hierarchies when the rate of intrazonal demand is low (figure 5.20).

On highway roads, arterial roads, and major collectors, method 4.2 outperforms method 4.1 in simulations when the rate of intrazonal trips (RIT) is less than 20\%. On the contrary, method 4.1 outperforms method 4.2 when the rate of intrazonal trips is greater than $20 \%$. Out of 700 simulations, method 4.2 produces the lowest PRMSE in nearly 400 cases. On minor collectors and local streets, methods 4.2 seems to outperform method 4.1 in reproducing original link flows (figure 5.20). The PRMSE indicator of method 4.1 is greater than that of method 4.2 in 500 simulations over 700 . Method 4.1 seems however to reproduce more accurate results in coarse models with 100 zones.

Given the above results, it is clear that the overall assignment quality is improved by the new assignment procedures 4.1 and 4.2. This contribution is explored in detail with the GEH indicator in Appendix B. Similar conclusions are drawn from these results: the distribution of median GEH is similar to that of the PRMSE: methods 4.1 and 4.2 are found to improve the quality of assignment outcomes and to reproduce more accurately reference flows than the standard assignment. For highway, arterial, and major collector roads, method 4.1 has the lowest median GEH when the rate of intrazonal trips is greater than 20\% (Appendix B). Method 4.2 is more accurate on low road hierarchies and especially on category 5 and with detailed zonings.

\subsubsection{Statistical significance of results}

Findings are unequivocal about the contribution of new assignment strategies. In this section, we investigate the statistical significance of this contribution. Since our research is based on a stochastic simulation approach, namely a stochastic aggregation process as described in section Zoning, findings from this research can be statistically validated or rejected.

Different statistical tests have been run to answer the following question: Are the results of the three assignment strategies statistically different or are they just a matter of random noise?

In the case of average link flows, this question can be reformulated in a more statistical-sound way:

$H_{0}$ : The three traffic assignment strategies have equal means of link flows

Since the three assignment strategies have different variances, $H_{0}$ is tested using Welch's t-test instead of standard ANOVA or Student's t tests (Welch, 1947). This is a Student-like test that is more appropriate than the ANOVA test when the variance of the samples is not equal. The null hypothesis $H_{0}$ is rejected at a significance level of 0 for road categories from 2 to 5 . However, the equality of means between methods 0 and 2 can only be rejected at a level of 0.11 for road category 1 (figure 5.21). 


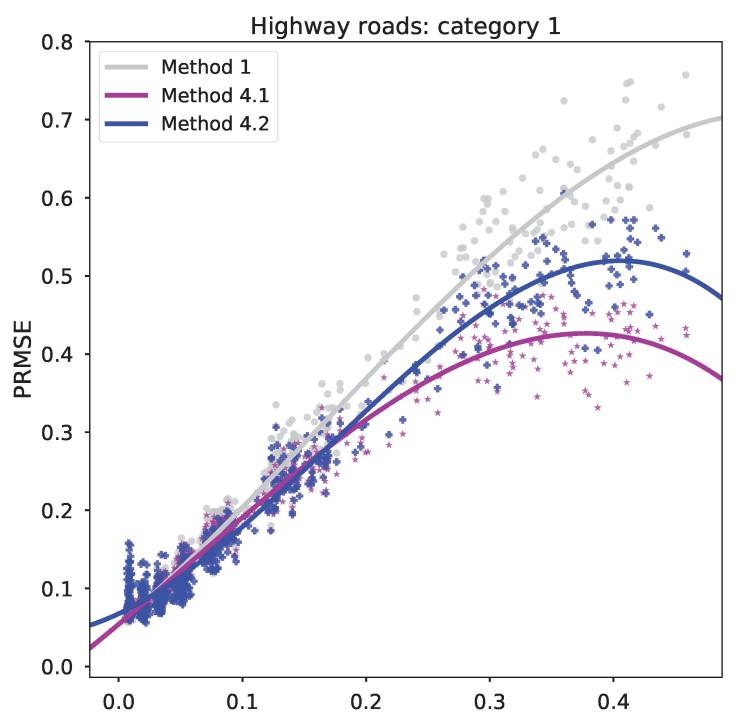

(a) Intrazonal trips rate

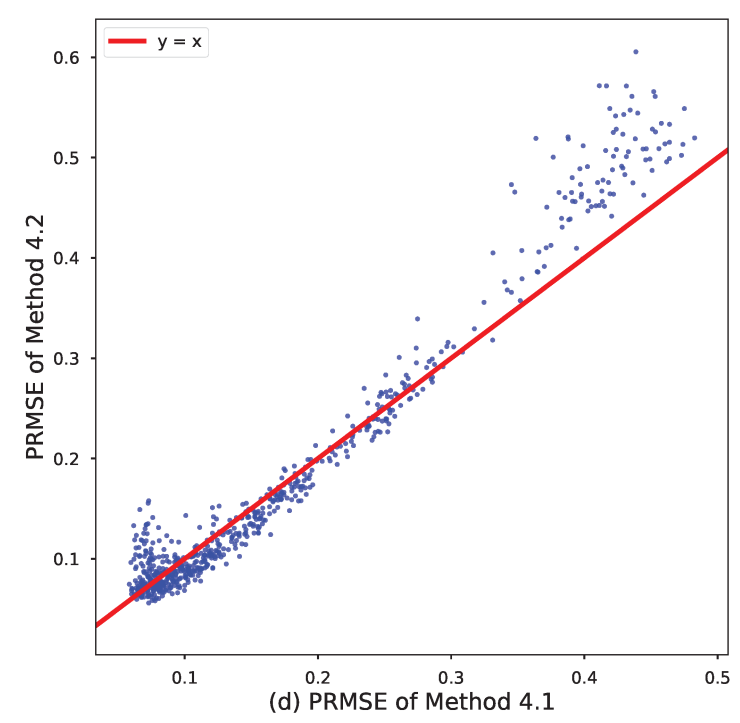

Arterial roads: category 2

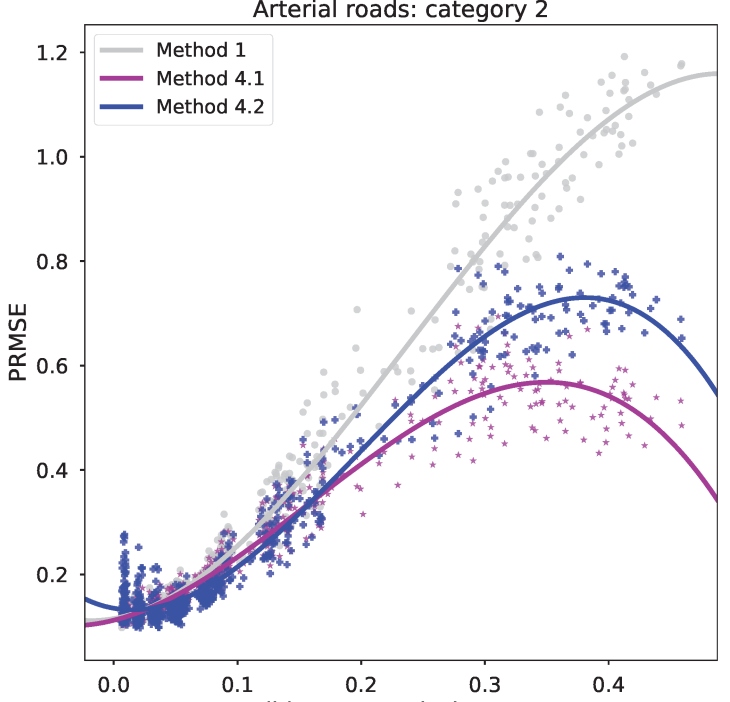

(b) Intrazonal trips rate

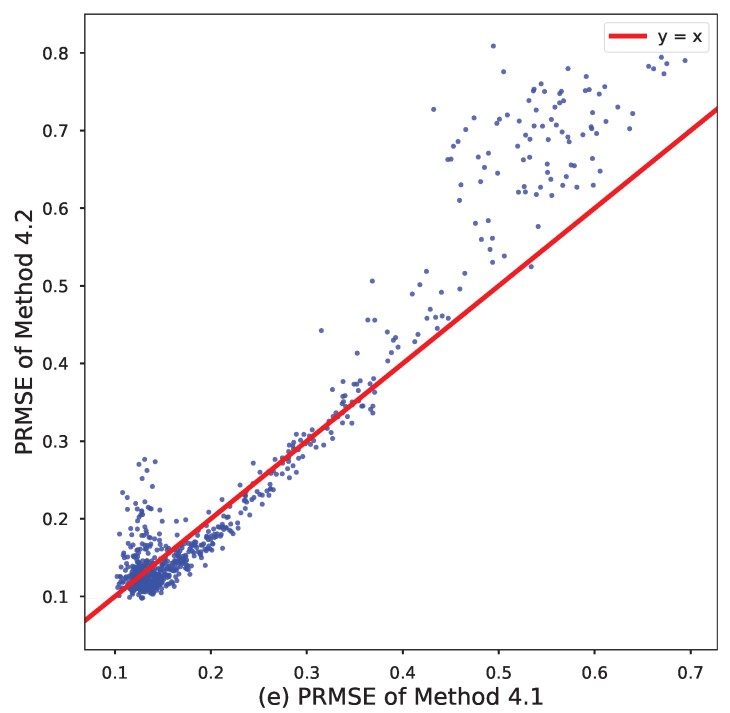

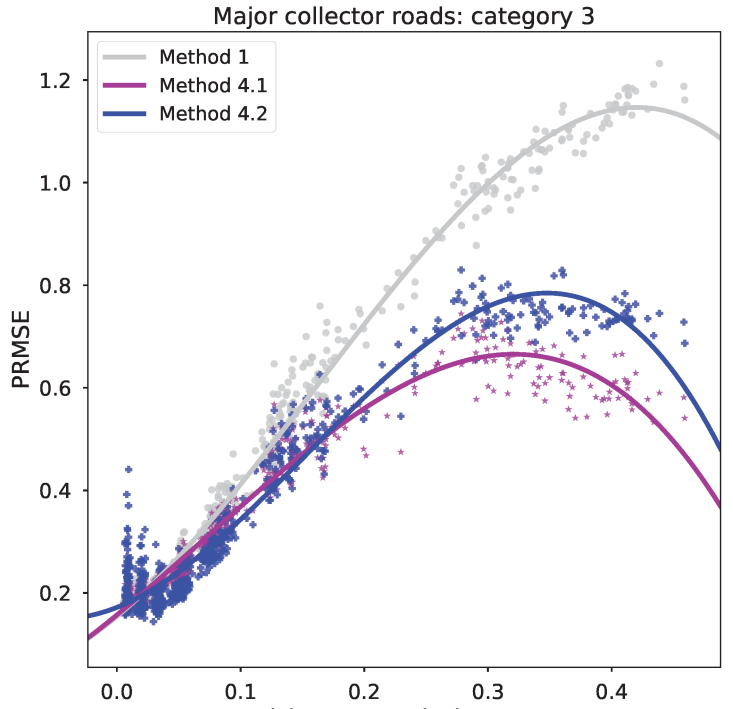

(c) Intrazonal trips rate

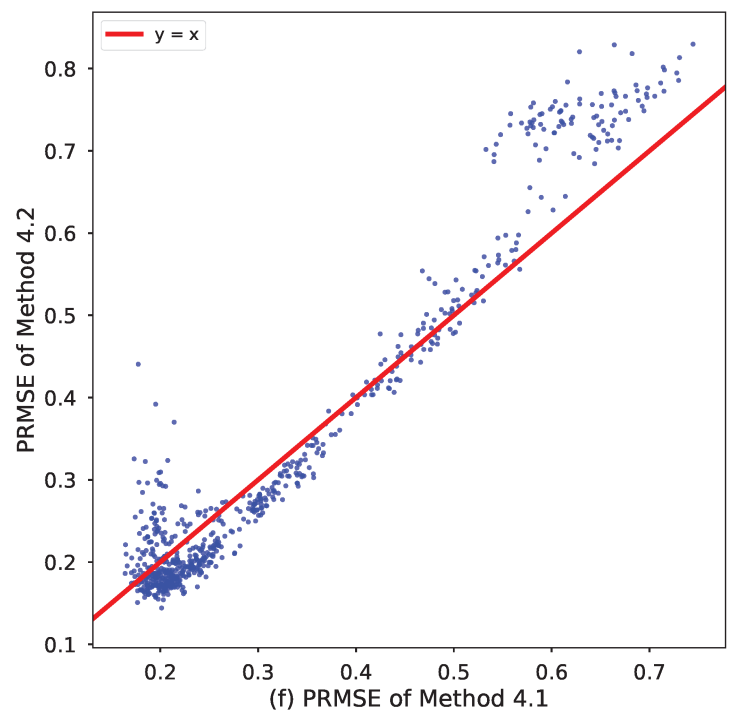

Figure 5.19: Lyon: PRMSE of link flows of methods 1, 4.1, and 4.2 on road categories 1, 2, and 3. Each point is a PRMSE value of an assignment simulation 

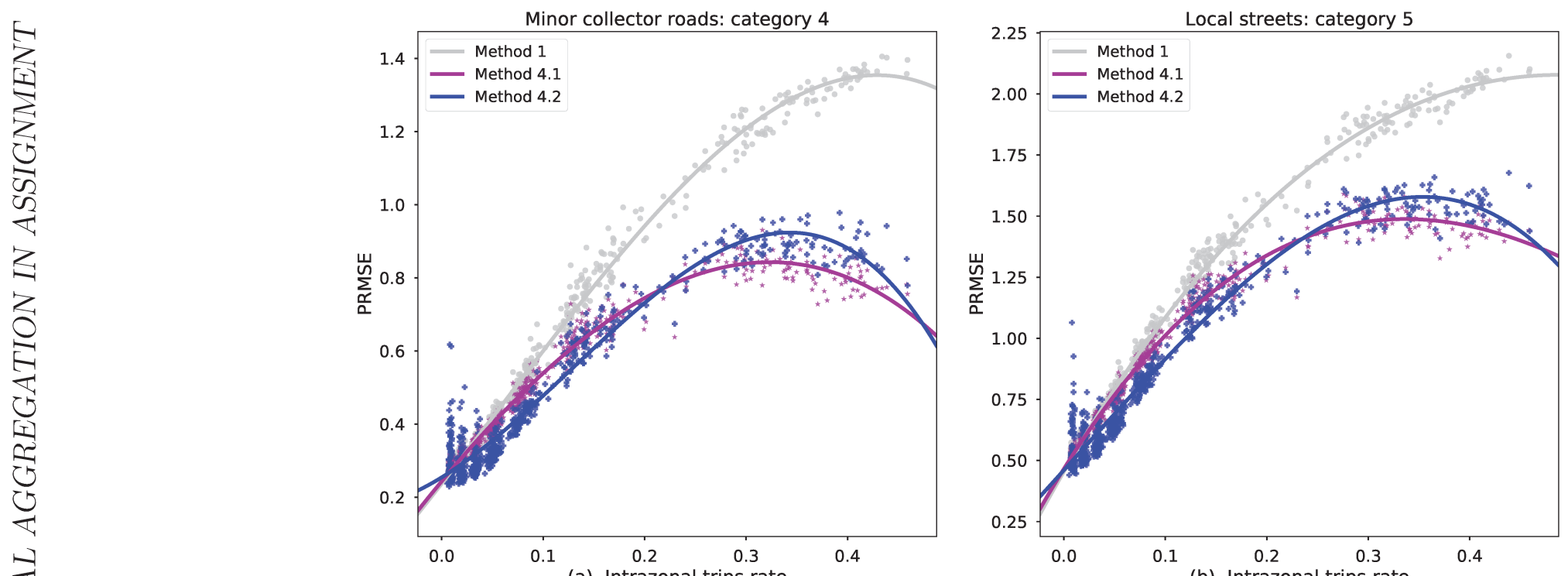

(a) Intrazonal trips rate
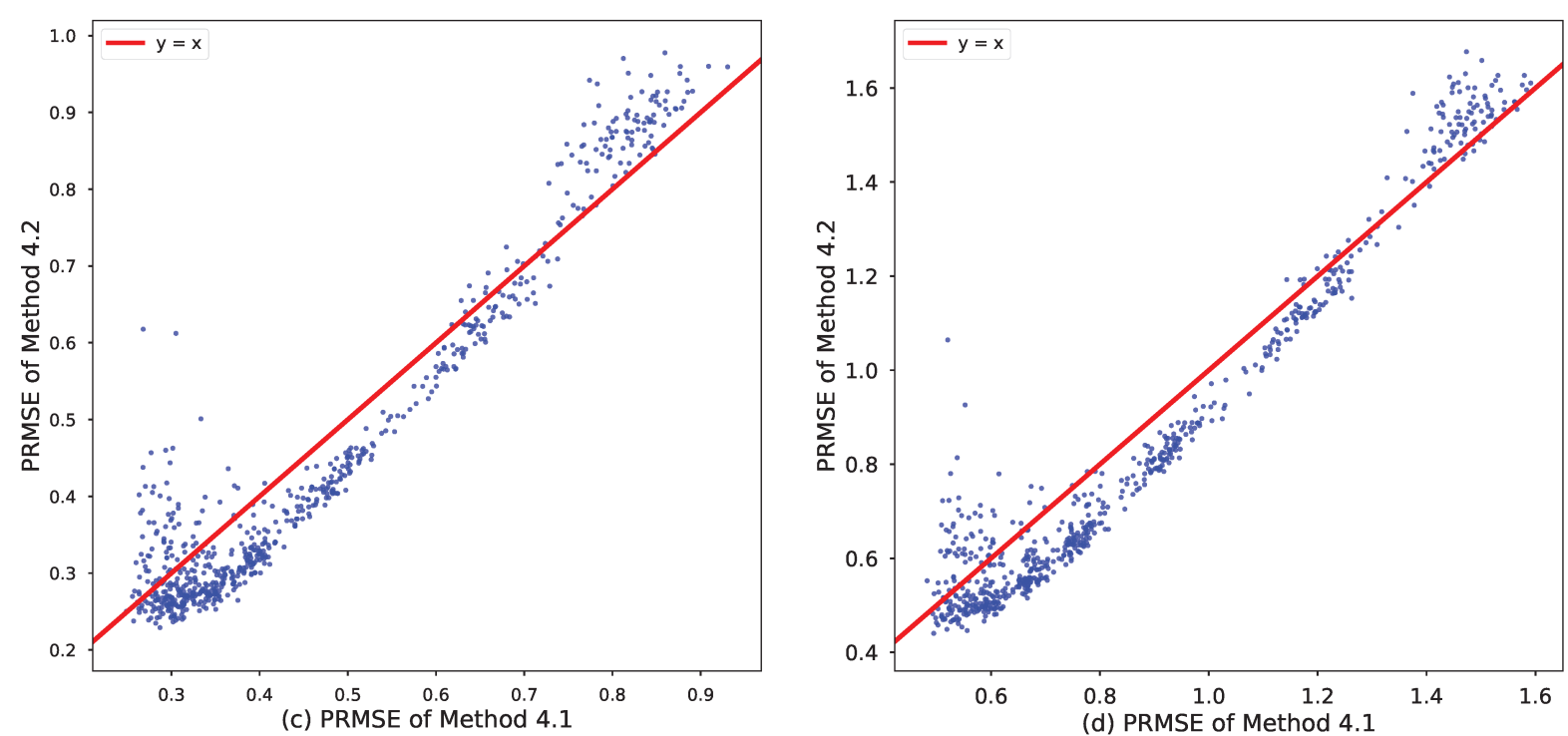

$\stackrel{\infty}{\stackrel{D}{\rightarrow}}$ Figure 5.20: Lyon: PRMSE of link flows of methods 1, 4.1, and 4.2 on road categories 4 and 5. Each point is a PRMSE value of an assignment simulation 

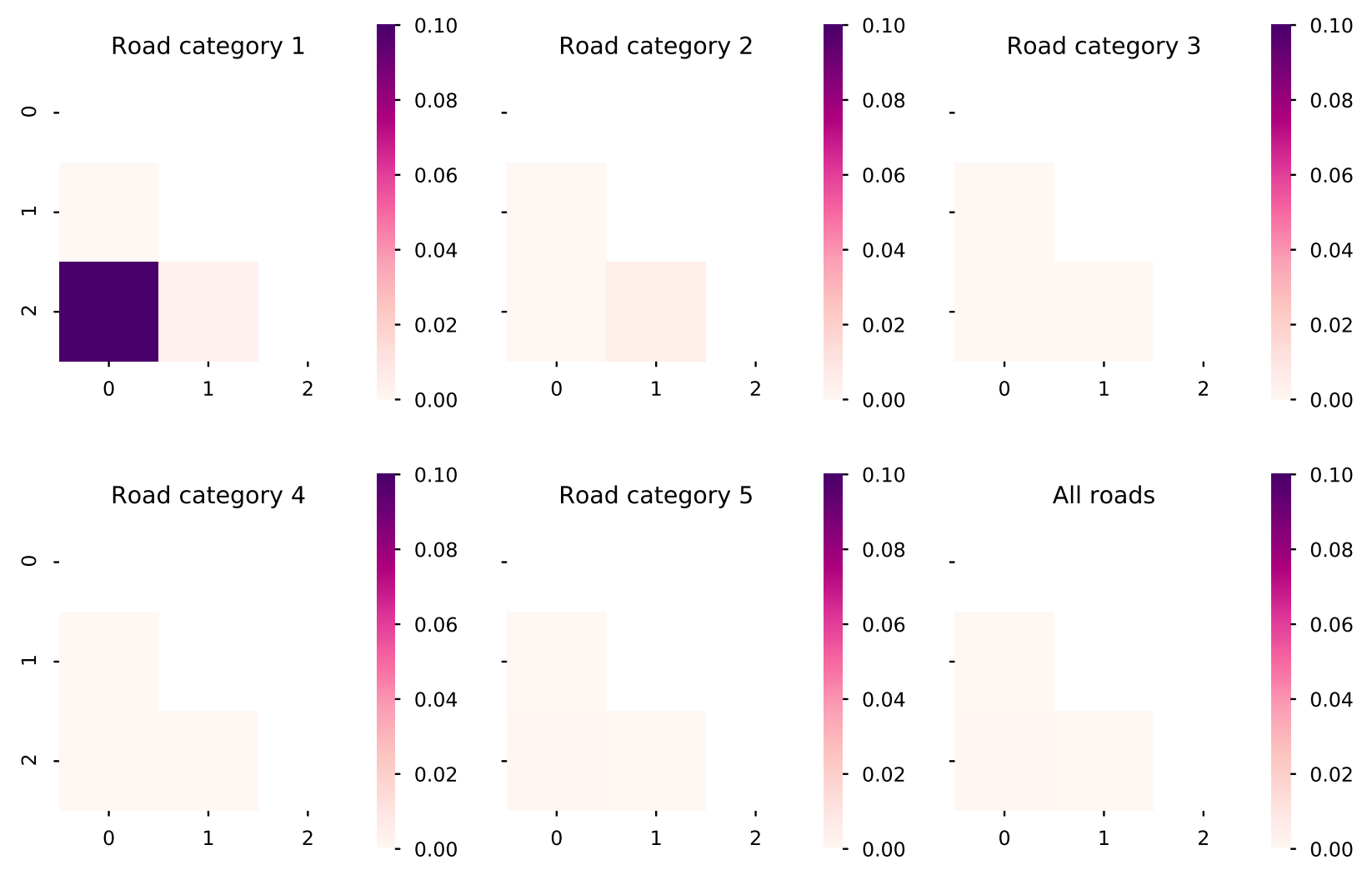

Figure 5.21: Lyon: significance levels of rejection of the Null Hypothesis of equal means of Welch's t-test

We have run the same tests on all accuracy measures (GEH, PRMSE), and all tests have statistically rejected the null hypothesis of equality. Meaning that, method 4.1 and 4.2 have a significant contribution to modeling results when compared to the traditional assignment.

\subsection{Discussion}

By including intrazonal trips in traffic assignment, methods 4.1 and 4.2 improve assignment outcomes. In comparison with the traditional assignment strategy, these methods produce more accurate results and less modeling errors. Both methods and especially method 4.1 is found to accurately reproduce original link flows. This method is based on simple assumptions using a uniform distribution of intrazonal demand and a uniform capacity on connectors. Results underline however a clear tendency of all assignment methods to underestimate average link flows and total Traveled Vehicle-Kilometers (VKT) despite the conservation principle of total travel demand. For methods 4.1 and 4.2, this underestimation is characterized by a parabolic shape. 


\subsubsection{Contribution of new assignment strategies}

Findings from up-to-date data of 2015, confirm those of chapter 4: assignment outcomes of the traditional assignment method are prone to bias. Main outcomes are severely biased by the omission of intrazonal trips and the rerouting of flows. Both effects contribute to a fictitious free-flow situation where congestion levels are underestimated and accessibility indicators overestimated. To correct for these shortcomings, assignment strategies 4.1 and 4.2 strive to assign intrazonal trips to the network and to reduce the impact of the rerouting problem. In this respect, main assignment outcomes, including average link flows and total Vehicle-Kilometers Traveled (VKT), become less subject to aggregation errors. Original outcomes are reproduced more accurately by new assignment strategies than by the conventional assignment. These findings hold true for all road hierarchies and even when ad hoc distribution methods like the uniform distribution of intrazonal demand or that of the capacity of connectors, are used. New assignment strategies have proven their contribution in addressing the aggregation bias and lessening its severity; nevertheless, these strategies are still subject to the aggregation bias and particularly to the elusive problem of rerouting.

\subsubsection{The rerouting problem}

One major finding of the current research is that the inclusion of intrazonal trips in assignment models does not address, by itself, the overall aggregation bias. Even when intrazonal demand is assigned to the network, main assignment outcomes are still subject to the fictitious free-flow bias. There is still a need to understand why new assignment strategies are still subject to bias.

Methods 4.1 and 4.2 assign all original travel demand, including intrazonal trips. No trip is omitted from assignment and yet traffic assignment outcomes are underestimated. The reason behind this bias is the definition of centroid connectors. The aggregation of zones and thus of centroid connectors, as described in section Zoning, introduces new routes to the routing tree as aggregation is performed. In fact, the aggregation procedure allows each macro-zone to use all its subzone connectors, meaning that macro-zonal flows have more available routes to access their destinations than before aggregation. Therefore, flows are likely to be rerouted from congested routes to uncongested ones after aggregation. The underestimation of link flows and VKT is then a matter of route choice modeling. Figure 5.22 illustrates clearly the rerouting problem. 


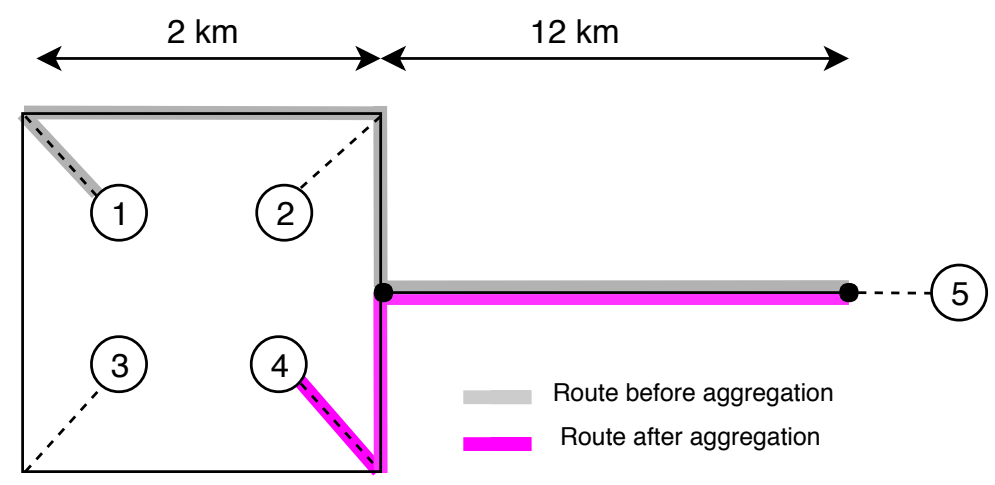

Figure 5.22: A sketch example of the rerouting problem

In this example (figure 5.22), travel demand from zone 1 to 5 is of $10 \mathrm{veh} / \mathrm{h}$. These trips must travel a distance of $15 \mathrm{~km}$ (excluding connectors) to access their destination. After aggregation of zones $1,2,3$, and 4 . The same demand can access to zone 5 through a route of only $13 \mathrm{~km}$ (excluding connectors). Accordingly, total VKT declines from 150 vek.km before aggregation to 130 veh.km after aggregation. This decline occurs despite the conservation of total demand $(10 \mathrm{veh} / \mathrm{h})$. In the same vein, average link flows ${ }^{6}$ decreases from $7.5 \mathrm{veh} / \mathrm{h}$ to $6.5 \mathrm{veh} / \mathrm{h}$. This result is due to the definition of connectors. As demonstrated in the simple case study, setting a finite capacity on centroid connectors and a restrictive volume-delay function does, at best, reduce the extent of this problem but not address it.

In the case study of Lyon, the rerouting problem is demonstrated in methods 4.1 and 4.2 by a decline in the Average Traveled Distance (ATD) (eq. 5.8, figure 5.23).

$$
V K T=A T D \times \text { Demand }
$$

${ }^{6}$ Average network flows are weighted by the length of links in order to minimize any statistical bias that may arise from network coding methods. 


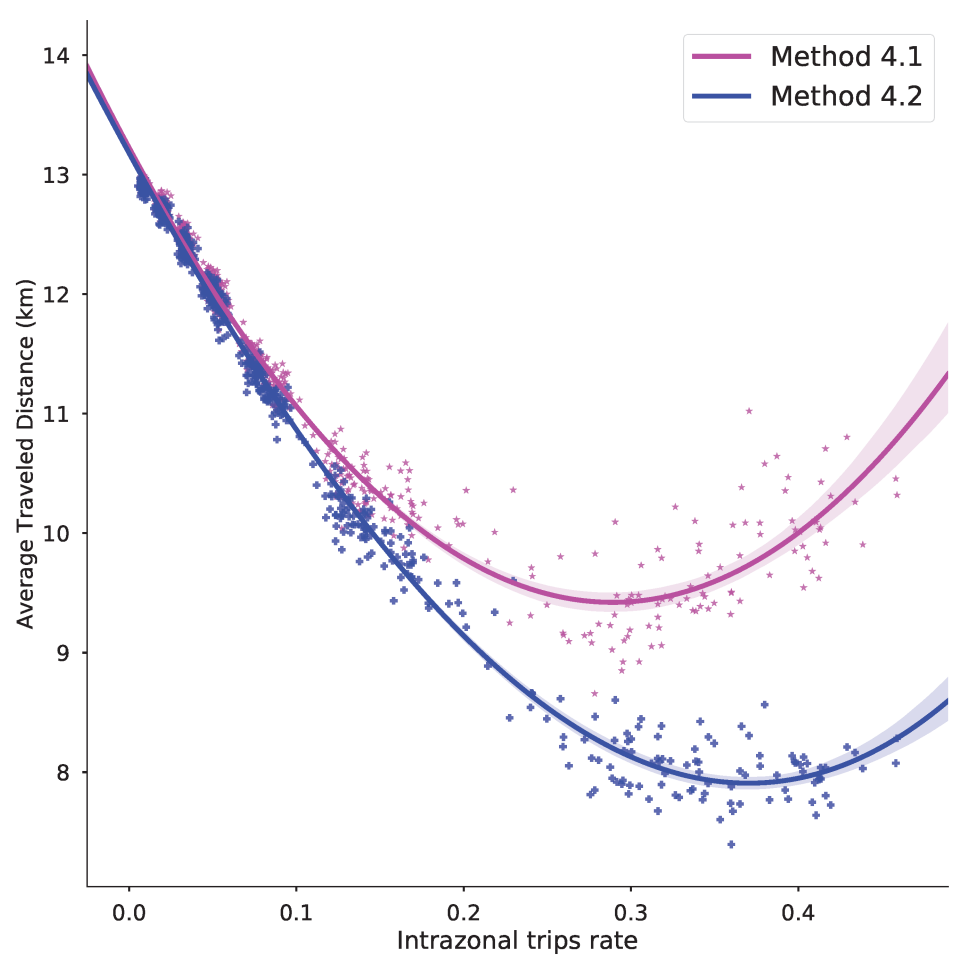

Figure 5.23: Lyon: Average Traveled Distance in Km function of intrazonal trips. The X-axis represents the rate of intrazonal trips and $\mathrm{Y}$-axis depicts the average distance traveled by cars in kilometers

For assignment method 4.1, 4.2, the Average Traveled Distance (ATD) decreases from $13 \mathrm{~km}$ to 10 $\mathrm{km}, 8 \mathrm{~km}$, respectively, when the rate of intrazonal trips increases (figure 5.23). This drop is due to a rerouting of flows from congested routes to uncongested ones. Accordingly, cars travel shorter distances after aggregation to reach their final destinations, which in turn induces a decrease in average link flows and total VKT.

Another reason that might also explain the persistence of the rerouting problem may come from the original model of Lyon. In fact, in this model, original centroid connectors are defined using the standard constrained definition where a maximum number of 10 connectors and a maximum length of 1,500 $\mathrm{m}$ are set. Therefore, the constrained definition of connectors may produce biased original network flows. The average traveled distance of original network flows is likely to be artificially higher than observed data due to the low number of connectors. That is why, the original model is too sensitive towards the definition of connectors and the rerouting of flows. As aggregation goes on, more connectors are included and a drop in average link flows and total VKT is observed. To sum up, the rerouting problem is due to the large number of connectors of macro-zones relatively to the low number of connectors in the original model. One possible solution to this problem is to use a definition of connectors that is more robust towards spatial aggregation as that of Manout et al. (2018) suggested for transit models. Such definition, should be as independent as possible from the 
spatial design by relying more on intrazonal data rather than the position of zone centroids. For lack of time, the author could not apply the new definition of centroid connectors, developed in chapter 3, to the present case study. Further research in this regard is promising and can help understand the last enduring aggregation bias due to connectors.

As aggregation goes on, one might expect a steady decrease in average traveled distance since more connectors are aggregated. Assignment results show a different pattern: assignment outcomes start, indeed, by decreasing till a certain point of aggregation before they increase (sub-figures $b, c, d, e$, and $f$ in figure 5.17). This pattern depicts a parabolic shape that is especially prevalent in method 4.1. The next section discusses the reasons behind such a pattern.

\subsubsection{Why a U-shape?}

Results bring to light a surprising parabolic shape in the distribution of average link flows and total Vehicle-Kilometers Traveled (VKT). The U-shape has been observed only in results produced by traffic assignment method 4.1 and to a lesser degree in 4.2 (figure 5.24). Standard assignment outcomes seem not to follow such a distribution.

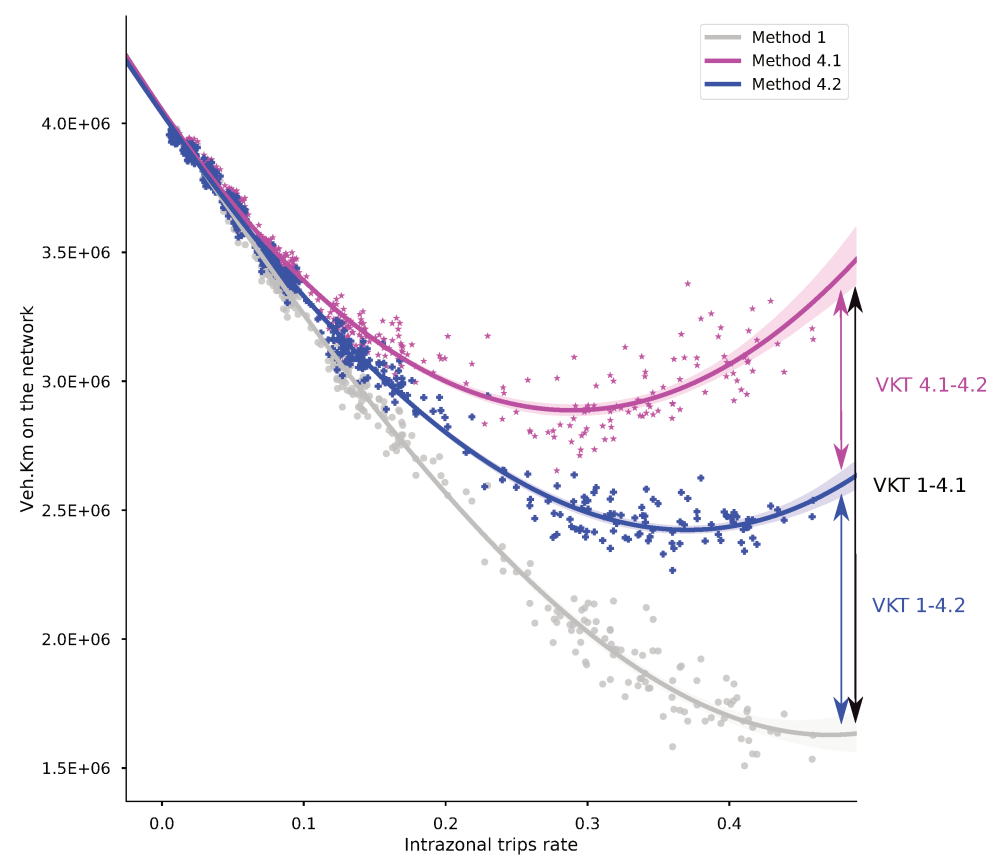

Figure 5.24: lyon :Total Vehicle-Kilometers of Travel on the network

The total distance traveled by cars (VKT) can be divided into two distinct parts: the total distance traveled by interzonal trips $\left(V K T_{\text {inter }}\right)$ and the total distance traveled by intrazonal trips $\left(V K T_{\text {intra }}\right)$ (eq. 5.9). For method 1, the intrazonal part is null. The U-shape is due to a trade-off between opposite forces underlying these parts. 


$$
V K T=V K T_{\text {inter }}+V K T_{\text {intra }}
$$

$V K T_{\text {inter }}$ is the total distance traveled by interzonal demand. Intuitively, this quantity decreases as aggregation goes on. As zones are aggregated together, the rate of intrazonal trips increases, i.e. more trips become intrazonal, and thus fewer trips are assigned to the network. Consequently, the number of vehicles considered for the computation of $V K T_{\text {inter }}$ drops. Furthermore, with aggregation, new connectors are defined for macro-zones, which, in turn, induce a rerouting of interzonal trips from longer to shorter routes. Therefore, a drop in Average Traveled Distance (ATD) is also observed (figure 5.23). To sum up, when the rate of intrazonal trips increases, the number of assignable trips decreases as well as their average traveled distance. This means that $V K T_{\text {inter }}$ is a decreasing function of intrazonal trips. In figure 5.24, VKT of method 1, which is the total distance traveled by cars in the standard assignment, can be also interpreted as the total distance traveled by interzonal demand given the unconstrained definition of centroid connectors. VKT 1 is, indeed, a decreasing function of intrazonal trips (figure 5.24).

$V K T_{\text {intra }}$ is the total distance traveled by intrazonal trips in methods 4.1 and 4.2 given the constrained definition of the capacity of connectors and the distribution of intrazonal demand. The new assignment strategies are designed to assign intrazonal trips and therefore $V K T_{\text {intra }}$ is non null. $V K T_{\text {intra }}$ is an increasing function of intrazonal trips: as aggregation is performed, the number of intrazonal trips increases as well. Moreover, as aggregation goes on, macro-zones extend and so does the average traveled distance of intrazonal trips. Finally, the distribution method of intrazonal demand has also an impact on the Average Traveled Distance of intrazonal trips. This impact is discussed in the next section. All things considered, $V K T_{\text {intra }}$ should increase as the rate of intrazonal trips increases.

All in all, for methods 4.1 and 4.2, VKT is the sum of two functions that have opposite tendencies: a decreasing and an increasing one. The U-shape derives from this trade-off (figure 5.25).

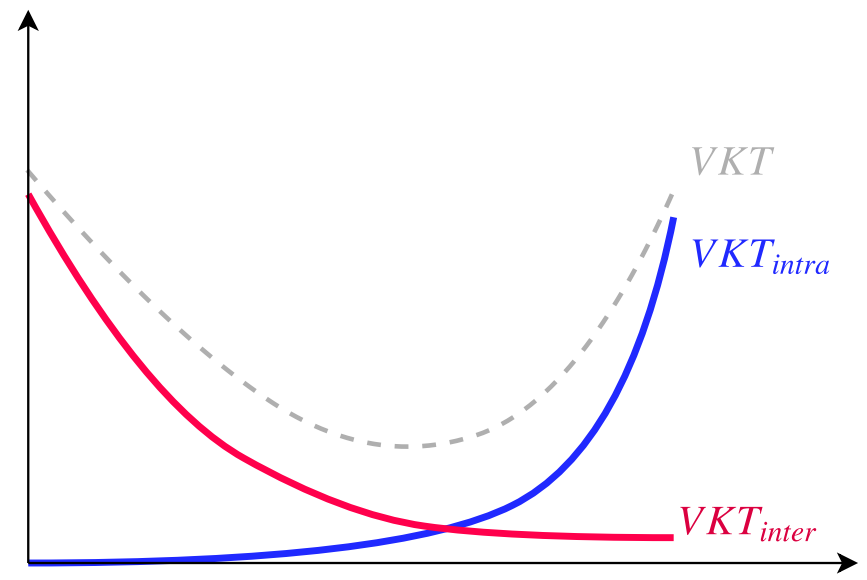

Figure 5.25: The U-shape of assignment outcomes 
The same conclusions hold for average network flows since these quantities are proportional to total VKT as suggested by equation 5.7.

\subsubsection{The artifact behind the outperformance of method 4.1 over 4.2}

Results from the case study of Lyon bring forward a remarkable finding: method 4.1 seems to be less subject to the free-flow bias than method 4.2 as suggested by figure 5.24 . In fact, outcomes of method 4.1 seem to be less prone to the drop in average link flows and total Vehicle-Kilometers of Travel (VKT) than method 4.2. Total VKT of method 4.1 is often greater than that of method 4.2 and therefore its outcomes seem to reproduce more accurately original observations. In this section, we uncover the reasons behind this finding.

Despite the fact that both methods assign the same total demand, method 4.1 often produces higher VKT and average link flows than method 4.2. This means that, in average, cars travel longer distances with assignment method 4.1 than 4.2. In other words, the Average Traveled Distance (ATD) in method 4.1 is higher than that of method 4.2. Our results confirm this observation (figure 5.23) and the reason behind this result is due to the distribution of intrazonal demand. In method 4.1, intrazonal demand is distributed uniformly between subzones; in method 4.2, the original distribution of intrazonal demand is used. The uniform distribution implies that all subzones, even those far from each other, exchange intrazonal trips with no regard to the distance between them; whereas the distribution of original intrazonal trips is likely to follow a gravity interaction schema where the intensity of interaction between subzones is inversely proportional to the distance separating them. In this regard, the use of a uniform distribution induces an artificial increase in the average traveled distance of intrazonal trips especially in coarse models. As spatial aggregation goes on, the size of macro-zones expands together with the ATD of intrazonal car trips produced by method 4.1 .

To test the sensitivity of average assignment outcomes (VKT and average link flows) to the distribution method of intrazonal trips, two new experiments are designed (table 5.23). Test 4.3

substitutes the original distribution for the uniform distribution in method 4.1. Test 4.4 switches the distribution of intrazonal trips from original to uniform in method 4.2. The definition of centroid connectors in method 4.3, 4.4 is the same as that of method 4.1, 4.2, respectively. These tests are run on an assignment model with 100 zones and a rate of intrazonal trips of $39 \%$. Results are compared to the original assignment. 
Table 5.23: Lyon case study: additional tests to explore the sensitivity of assignment results towards intrazonal trips distribution

\begin{tabular}{llll}
\hline Test & Graph & Connectors & Demand \\
\hline 4.3 & Original graph & Finite \& uniform capacity & Original intrazonal demand \\
4.4 & Original graph & Finite \& original capacity & Uniformly distributed intrazonal demand \\
\hline
\end{tabular}

According to our assumptions, we expect that test 4.3, 4.4, will produce similar results to method 4.2, 4.1, respectively. Assignment results are concordant with this assumption: the use of a uniform distribution of intrazonal car trips induces an increase in average link flows and total VKT in method 4.4 independently of the definition of the capacity of centroid connectors (table 5.24).

Table 5.24: Assignment results of tests 4.3 and 4.4 compared to original results of methods 4.1 and 4.2

\begin{tabular}{llll}
\hline Tests & VKT (veh.km) & Link Flows (veh/hour) & ATD $(\mathrm{km})$ \\
\hline 4.1 & $3.173 \mathrm{E}+06$ & 152.90 & 17.03 \\
4.4 & $3.085 \mathrm{E}+06$ & 148.66 & 16.56 \\
4.2 & $2.405 \mathrm{E}+06$ & 115.91 & 12.91 \\
4.3 & $2.506 \mathrm{E}+06$ & 120.80 & 13.45 \\
\hline
\end{tabular}

Therefore, the apparent outperformance of method 4.1 over 4.2 in reproducing average original outcomes is a matter of bias induced by the distribution method of intrazonal trips.

\subsection{Conclusion and perspectives}

Traffic assignment models often rely on an aggregate description of space. This description is found to be a serious source of bias in standard traffic assignment models. The traditional assignment has already been pointed out to be prone to aggregation errors and to produce biased results (Manout and Bonnel, 2018). Yet, this model is still popular and in wide use in both academia and practice. The aim of the current research is to develop new traffic assignment strategies that are, first, practical and, second, robust towards the aggregation bias. To this end, 6 different assignment strategies are tested and investigated in different case studies. These methods are designed to tackle two of the major spatial aggregation errors: omission of intrazonal demand and rerouting of flows due to connectors. Both errors are found to be detrimental to the accuracy and reliability of assignment outcomes. At one hand, the omission of intrazonal trips from assignment induces a free-flow situation where accessibility indicators are overestimated and congestion levels underestimated. At the other 
hand, the rerouting of flows due to the definition of centroid connectors, adds to the free-flow situation by rerouting artificially flows from congested routes to uncongested ones. To correct for these shortcomings, 6 different strategies are investigated in detail. These strategies can be divided into two categories:

1. Supply-side approaches: include the omission of intrazonal trips in the definition of the network (method 3: capacity reduction of intrazonal links).

2. Demand-side approaches: include intrazonal demand by using a subdivision of zones (method 4: subdivision around zones).

Findings of this research are twofold:

- The inclusion of intrazonal demand in traffic assignment improves modeling results even when ad hoc distribution methods are used.

- The inclusion of intrazonal demand is not sufficient to overcome the spatial aggregation bias. The rerouting problem is still persistent even after restraining the definition of centroid connectors.

Demand-side methods outweigh both the supply-side approach and the standard assignment in many respects. Demand-side methods are found to significantly improve the accuracy of assignment results and to reduce the bias introduced by aggregation in the standard traffic assignment.

Another worthwhile finding is that to produce accurate assignment outcomes, one does not need to use special data. Using a uniform distribution of intrazonal demand and a uniform definition of centroid connectors brings, by itself, a satisfactory contribution to main assignment outcomes. This finding is of importance in order to design practical and useful assignment models as our research strives to. Whatever spatial aggregation level is used, it is possible to assign intrazonal trips to the network and to get rid, or at least, to reduce the omission bias by using assignment strategy 4.1. Nonetheless, despite this contribution and our effort to tackle the aggregation bias, findings suggest also that modeling results of new assignment strategies are still subject to bias. Part of this bias is still due to aggregation (rerouting); the other part is due to some first-cut assumptions we have made (distribution of intrazonal trips).

The inclusion of intrazonal trips in assignment models is not a sufficient condition to get rid of the aggregation bias. Modeling outcomes are still prone to a problem that has been disregarded by previous research: the rerouting of trips due to the definition of centroid connectors. As aggregation is performed, the number of connectors of macro-zones increases since all subzonal connectors are conserved. New routes that have not been included in the original model are then introduced by these connectors. In many configurations, these routes are used by aggregate demand to bypass longer or congested routes. As a consequence of the rerouting problem, average link flows and total vehicle-kilometers drop unavoidably with aggregation even if total demand is held constant. To 
correct for this issue, a constrained definition of the capacity of centroid connectors is used. In this way, each connector can only convey a limited number of car trips and travel demand is, in turn, distributed between numerous connection nodes. The constrained definition of capacity has also the advantage of preventing fictitious congestion in the vicinity of centroid connectors. This ad hoc solution boosts the accuracy of modeling results and reduces to some extent the impact of rerouting; however, outcomes of our experiments still reveal a significant effect of the rerouting problem. That is because, a constrained definition of the capacity of centroid connectors is not an effective solution to the rerouting problem.

One possible solution to the rerouting problem is the use of the proportional assignment, i.e. subdivision around connectors (Mann, 2002). Each traffic zone with $n$ connectors is subdivided into $n$ different subzones. Each subzone has only one connector. The corresponding proportional trip matrix is computed using a predefined distribution method and assigned to the network. In this manner, each travel demand proportion can access and egress the transportation network using one and only one directed connector. In this case, the rerouting problem is less likely to occur than with previous assignment strategies. Nevertheless, this method requires the definition of a detailed trip matrix especially when zones have a non marginal number of connectors (CBD zones for example). In this regard, the relative advantage brought by this method may be offset by the need to disaggregate the trip matrix, especially in predictive situations. Furthermore, the use of connectors as a subdivision basis, makes the proportional assignment dependent on the definition of connectors and potentially biased by any error in their definition. Further research is needed in this regard.

Another question raised by this research pertains to the definition of the trip matrix. To include intrazonal trips in traffic assignment models, intrazonal demand must be included in the trip matrix and distributed between subzones. To this end, one needs a partitioning method of demand between subzones and a distribution method of intrazonal demand between these subzones. In the three previous case studies, the question of zone subdivision has not been addressed since original subzones have been used. In practical situations, this is not often the case. To this end, two different strategies can be used:

1. Manual subdivision of zones: each zone can be divided according to some rules-of-thumb set by the modeler. This is cumbersome and might be prone to bias.

2. Automatic subdivision of zones: these methods rely on subdivision criteria or objective functions to construct new spatial divisions. These methods have already been discussed in literature and their bias can be controlled for using the simulation framework (Baass, 1981; Horowitz, 2001; Mann, 2002; Manout and Bonnel, 2018; Martínez et al., 2009; Openshaw, 1977b; O'Neill, 1991). Further research needs to be undertaken to assess the contribution of these methods under the light of the spatial aggregation problem in assignment models.

The study of distribution methods of intrazonal demand is also of interest. As suggested by the case 
study of Lyon, the distribution method of intrazonal trips has a direct impact on main assignment results. For instance, a uniform distribution of intrazonal trips is found to falsely increase the average traveled distance when compared with the original distribution. Choosing a distribution method is therefore not straightforward especially when the spatial resolution of the model is coarse. For simplicity purposes, only the uniform distribution has been examined in this research; yet other practical methods can be used: gravity models (Hansen, 1959), intervening opportunities (Stouffer, 1940), radiation models (Simini et al., 2012), or discrete choice models (McFadden, 1978). This question needs further research to investigate the contribution of new distribution techniques more suitable for the distribution of short distance migrations like that of intrazonal trips.

Finally, our research design keeps constant the definition of the transportation network. This choice is made to isolate, as much as possible, the aggregation bias from other errors like those raised by the definition of the network. By doing so, other problems may occur especially from the lack of consistency between the spatial resolution of the model and its network. To avoid such inconsistencies, one needs to adapt the definition of the transportation network to the spatial design and vice versa. Most transport models still adopt the implicit assumption of "the more details you include, the better results you get". This assumption is a starting point question in the network aggregation problem. Similar to the spatial aggregation problem, the network aggregation problem addresses the question of modeling transport networks (both their representation and functional description) and the implications of these choices on modeling outcomes and computation costs (Chan, 1976; Connors and Watling, 2014, 2008; Haghani and Daskin, 1986, 1983). These research questions, i.e. the spatial aggregation and the network aggregation problems, are interdependent; yet, seldom are the studies that have addressed the problem of consistency between spatial and network modeling (Bovy and Jansen, 1983; Friesz, 1985). Further research at the junction of these two problems may bring interesting insights to the problem of aggregation in transportation models.

\section{Acknowledgments}

The author is grateful to François Pacull for his help with the traffic assignment computer program. Without the support of François, assignment simulations could not be undertaken. The author is also thankful to ForCity for supporting this work and managing no efforts to help him achieve this research. 


\section{Chapter 6}

\section{General discussion}

\subsection{Thesis overview}

Land-Use and Transport Interaction (LUTI) Models are valuable tools to understand cities' dynamics and complexity. By their very nature, LUTI models can account for various interaction processes underlying urban organization and changes. Understanding and unrevealing these processes is a noteworthy goal that research has actively sought. In this regard, many research projects have engaged in pushing forward our understanding of the urban phenomena. Consequently, urban models have witnessed a thriving sophistication especially within the microsimulation modeling framework. Nonetheless, these efforts often come at a price of increased complexity that only few research institutes and LUTI specialists can handle. This is a serious entry barrier that prevents LUTI models from wide dissemination. In this regard, LUTI models fail to meet one of their primary objectives: becoming practical decision-support tools in urban planning. Meanwhile, decision-makers and urban planners are seeking for practical tools to help them cope with their daily missions. In fact, urban planning often involves different stakeholders with various and differing goals. The implications of any urban planning scheme or project are often complex to comprehend if not counter-intuitive: they can evolve in space and time and embrace complex interaction chains. In this context, LUTI models can be invaluable, provided that these models become practical. Efforts in this regard are scarce and are often considered out of research scope ${ }^{1}$.

The current $\mathrm{PhD}$ research is undertaken within a broad research project that aims at enhancing the practicality of LUTI models for urban planning purposes. Within this broad research framework, we assume that a special attention should be devoted to practicality issues ${ }^{2}$ in LUTI models and that the standard modeling framework is an appropriate modeling background to achieve such a

\footnotetext{
${ }^{1}$ While major research streams focus on the theoretical and empirical sophistication of LUTI models, the simplification of these models can be considered by some academics and journals as out of research scope.

${ }^{2}$ By practical we mean useful, replicable, affordable, and responsive models.
} 
goal. Given these assumptions, the current $\mathrm{PhD}$ investigates the spatial aggregation problem in standard LUTI models with a focus on traffic assignment models. In contrast with other modeling frameworks, standard LUTI models are a promising framework that complies, more or less suitably, with practicality objectives. This framework relies on an aggregate description of agents, time, and space. By doing so, standard models reduce, to some extent, urban complexity while still delivering useful insights with relatively reasonable costs. Nonetheless, by their very nature, these models are subject to aggregation errors. This $\mathrm{PhD}$ is committed to the study of one of these errors: the spatial aggregation problem. The spatial aggregation problem stems from the use of a discrete description of space using zones instead of a continuous description. This problem has long been disregarded by economists, even if it is deemed to induce specification errors and to undermine the reliability of modeling results. Consequently, the spatial aggregation problem is detrimental to the usefulness and, thereby, to the practicality of LUTI models and needs to be addressed. In transportation modeling, the use of an aggregate spatial description has two main implications:

- The definition of centroid connectors to attach zone centroids to graph nodes.

- The omission of intrazonal trips in assignment models.

By relying on a discrete description of space instead of a continuous one, individual locations of trip makers are aggregated to the zonal level of resolution and summarized by centroids. Centroid connectors are used to attach zone centroids to the transportation network. The majority of transportation models use ad hoc methods to define centroid connectors: zone centroids are attached to the nearest graph nodes using dummy links. The maximum number of these dummy links and their maximum length are also set on an arbitrary basis.

The use of zones as basic spatial units induces a loss of intrazonal information. Intrazonal trips are an example of such a loss. Intrazonal trips are trips that start and end at the same zone. For this reason, these trips are discarded form traffic assignment models. The deliberate omission of these trips induces an omission bias and a fictive free-flow situation where congestion levels are underestimated and accessibility scores overestimated. This bias undermines the accuracy and the reliability of modeling results and therefore their usefulness for decision-making.

To address these problems, this research has been assigned two main objectives: (1) Investigate the extent of the bias induced by the aforementioned problems; (2) Develop new modeling strategies to address these problems. To this end, three research questions are examined:

1. How space is modeled in traffic assignment models?

2. What are the implications of these modeling choices on assignment modeling results quality, accuracy, and reliability?

3. What will be the contribution of new modeling strategies that account more explicitly for spatial aggregation in traffic assignment? 
These questions are addressed in 4 chapters. General findings and recommendations from these chapters are summarized in the next section.

\subsection{Contribution and recommendations}

\subsubsection{A new definition of transit connectors}

\subsubsection{Thesis contribution}

In Chapter 2, a sensitivity analysis is undertaken to assess the impact of using the standard definition of centroid connectors on modeling outcomes. The standard definition of connectors relies on both the geographic position of centroid connectors and ad hoc constraints on the maximum number and length of connectors. Hence, this method is subject to the spatial aggregation bias. Any change in the size or shape of zones, or the position of their centroids induces a change in the definition of centroid connectors and ultimately a change in modeling outcomes (Chang et al., 2002; Jeon et al., 2012; Manout et al., 2018). Since the definition of centroid connectors is often arbitrary, this dependency has been shown to induce a serious bias in modeling results and especially in transit modeling. Main transit assignment outcomes including transit ridership and transfers are found to depend on the definition of centroid connectors.

The use of a constrained definition of centroid connectors is found to induce erroneous transit ridership rates especially in feeder transit systems, namely the bus. By restraining the maximum number and length of connectors to low values, as it is often the case in operational models, transit assignment models produce higher transfer and ridership rates than observed data. In this case, transit trips need to make numerous transfers between different transit systems to reach their final destination. In the case of Lyon, we found that the average number of transfers drops by nearly four times when the maximum number of connectors increases from 1 to 100 connectors. In practice, transport models often set low values for the maximum number and length of connectors: 5 or 10 connectors within 1,500 meters. This practice may be a serious source of modeling errors. These

errors are often covered-up during the calibration step by manually changing the characteristics of transit connectors, i.e. their travel times or connection nodes or other calibration parameters.

Furthermore, findings suggest that feeder transit systems, like the bus or rapid bus in the case of Lyon, are the most impacted systems by the definition of transit connectors. That is because, these systems are often used to enter and to exit the transit system. Connection nodes of centroid connectors are often attached to these systems and any bias in modeling transit connectors has a direct impact on modeling results of these feeder modes.

To overcome these modeling errors, chapter 3 introduces a new practical modeling method of transit connectors. This method is in line with four guidelines: 
- Generating transit connectors on the basis of actual remoteness from transit facilities.

- Generating transit connectors independently of the position of zone centroids.

- Reducing user intervention by automatically generating transit connectors.

- Removing the standard constraints used in operational models to generate transit connectors (maximum number and maximum length).

To this end, this method makes use of intrazonal data: population and jobs locations as well as their probability of using transit facilities conditional on their remoteness. The use of intrazonal data substitutes for the use of the geographic position of zone centroids. On the basis of these data, two methods are developed: (1) Choice of connection nodes; (2) Computation of the length of transit connectors. These methods allow for a selection of a sufficient maximum number of connection nodes that cover access and egress needs of potential transit users and for the computation of transit access times that translate actual remoteness of potential transit users from transit facilities. The application of this new definition on the case study of Lyon demonstrates that main transit assignment outcomes are improved. Observed data are more accurately reproduced when using intrazonal data than with the standard method. On the basis of these promising results, we have made available the source code of the computer program of this new method. This program allows for an automatic construction of centroid connectors and reduces the manual intervention of transport modelers. Two ongoing studies are testing the contribution of this new definition in two different transportation models of the Paris region: MODUS (DRIEA) and ARES (SNCF-Transilien). Results from these case studies are not yet available for analysis but are already a positive sign regarding the dissemination objective.

\subsubsection{Recommendations}

Two main recommendations can be drawn from the study of the spatial aggregation problem in transit access modeling:

1. When defining centroid connectors, one should rely, as far as possible, on intrazonal data instead of the geographic position of zone centroids.

2. In the absence of intrazonal data, one should relieve the constraints of the standard definition of centroid connectors, in order to connect each reachable transit facility.

The spatial aggregation problem arises from the aggregation or the omission of intrazonal data. Whatever aggregation function is used, this latter often induces a loss of information and therefore a potential bias. In the case of transit connectors, this issue is found to be prejudicial. The use of intrazonal data in the definition of connectors can reduce the extent of this bias. In this regard, the more accurate and detailed are the data the better the results are likely to be. Intrazonal data can reduce, to some degree, the problem of spatial aggregation by accounting for intrazonal variability. Nevertheless, these data are not always available. In this case, less detailed data are likely to have a 
significant contribution relatively to the crude standard definition. For instance, in the case study of Lyon, a synthetic population at the building level of resolution has been computed using simple disaggregation methods to substitute for actual population distribution (Manout, 2014). The use of this synthetic population has proven to be useful and to produce reliable modeling outcomes.

In the absence of any intrazonal data, one can still use the standard definition of centroid connectors and alleviate its bias. In this case, our findings show that the less biased results are produced when the number of connectors is unconstrained, namely high. When the number of connectors is high, transit demand is considered to have access to different transit facilities and transit routes. In the case of Lyon, findings from a cross comparison of different models using different configurations of connectors demonstrate that when the number of connectors is high, i.e. more than 20, transit modeling outcomes are more accurate than with restrictive configurations of centroid connectors. In fact, a special emphasis should be put on the definition and the connection of reachable transit facilities. Each zone should have access to all reachable transit facilities. One straightforward solution to this is to reduce the standard constraints on the definition of connectors. In this manner, one can get rid of the overestimation problem of transfer rates. Nonetheless, increasing the number of connectors has a computational $\operatorname{cost}^{3}$. In this case, a trade-off between the number of connectors, computational costs, and the accuracy of modeling outcomes is required.

\subsubsection{The impact of ignoring intrazonal trips in traffic assignment models}

\subsubsection{Thesis contribution}

Intrazonal trips are another manifestation of spatial aggregation. These trips start and end at the same zone centroid. They are therefore deliberately discarded from analysis and not accounted for in standard traffic assignment models. Yet, this omission is a serious source of bias. The existence of this bias, its extent, and its statistical significance are investigated in chapters 4 and 5 . In chapter 4, an uncertainty analysis approach is developed to characterize this impact and its statistical significance. The core idea of this approach relies on the observation that intrazonal trips are dependent on the spatial resolution of models. Any change in the spatial design induces a change in the rate of intrazonal trips and thereby a change in the assigned travel demand. Coarse spatial models are more likely to produce higher rates of intrazonal demand than detailed ones, everything else being equal. On the basis of this idea, different spatial divisions with varying degrees of spatial resolution are used to infer the statistical impact of the omission of intrazonal trips in standard traffic assignment models.

The standard traffic assignment model discards intrazonal trips from analysis: only interzonal travel

\footnotetext{
${ }^{3}$ In the case study of Lyon for instance, configurations with 99 connectors induce an increase of nearly $50 \%$ of computation times relatively to configurations with 10 connectors.
} 
demand is assigned to the network. The bias induced by this omission is proved to be detrimental to the accuracy and reliability of modeling results. Main traffic assignment outcomes including: link flows, link speeds, congestion ratio, and total Vehicle-Kilometers of Travel (VKT) are subject to the omission bias. These findings are statistically proved. The omission of intrazonal trips induces a fictive free-flow situation where accessibility indicators are overestimated and congestion levels underestimated. This is especially noticeable in coarse models and in low road hierarchies, i.e. local streets compared to other networks like motorways. That is because trips that are considered intrazonal by transport models, are more likely to use the secondary network than the primary one, and thereby their omission has a direct impact on these links. This is true up to a certain degree of aggregation beyond which the fictive free-flow bias spills over into high hierarchy roads as well. Average link flows and Vehicle-Kilometers of Travel (VKT), which are often used in transportation

and environmental analysis for policy-making purposes, are especially found to be significantly biased by the omission of intrazonal trips. This conclusion casts serious doubts on the reliability of main traffic assignment outcomes and brings forward concerns on the policy implications of such modeling errors (Bain, 2009; Flyvbjerg, 2007, 2005; Flyvbjerg et al., 2005).

Another noteworthy finding from this research shows the existence of a threshold effect in traffic assignment outcomes with respect to spatial aggregation. Traffic assignment outcomes are found to show signs of convergence when the rate of intrazonal trips falls, i.e. when the number of zones increases. Beyond a certain degree of details, further spatial resolution has limited if no contribution to the accuracy of modeling results. The marginal contribution of refining the spatial resolution of a model beyond this threshold can be inconsiderable with regard to its corresponding cost. This finding is in line with those of Bovy and Jansen (1983) and Ding (1998) who draw similar conclusions in different spatial contexts.

Chapter 4 brings to light another noteworthy spatial aggregation problem: the rerouting of flows due to the definition of centroid connectors. Chapter 4 does not disentangle the impact of rerouting from the issue of intrazonal trips omission. The study of the rerouting problem is carried out in chapter 5 .

\subsubsection{Recommendations}

Four major recommendations can be made on the omission of intrazonal trips in traffic assignment models:

1. When reporting modeling results, one should consider the impact of ignoring intrazonal trips.

2. When choosing spatial division of traffic models, one should prefer divisions that minimize intrazonal trips.

3. When choosing spatial division of traffic models, one should take advantage of the existence of the threshold effect. 
4. When analyzing traffic assignment results, one should avoid drawing conclusions from low road hierarchies.

The omission of intrazonal trips from traffic assignment models induces a bias in traffic modeling. As far as these models are used for decision-making purposes, the bias induced by such an omission should be either assessed or, at least, acknowledged. This is especially true for regional and metropolitan transportation models which often rely on coarse spatial designs. Link flows, congestion ratio, Vehicle-Kilometers of Travel, and to a lesser degree link travel times, are found to be significantly biased by intrazonal trips. If not addressed properly, this bias can mislead urban policy-makers and increase the risks of getting unpredictable social, environmental, and economical costs from urban projects. Therefore, it is important to address the intrazonal omission problem or at least to acknowledge its existence and potential impacts when reporting modeling results. To this end, the uncertainty framework used in this $\mathrm{PhD}$ has proven to be a convenient approach to statistically characterize this impact. At one hand, findings from this approach can be tested using common statistical analysis techniques to provide accurate and reliable conclusions and recommendations. At the other hand, the use of an uncertainty analysis framework, where most likely modeling configurations are tested, allows for a better and global understanding of the questions at hand.

Another solution to tackle the omission bias of intrazonal trips is to rely, as far as possible, on spatial divisions that minimize the rate of these trips. In this regard, the minimization of intrazonal trips has already been put forward as a spatial design criterion (Baass, 1981; Martínez et al., 2009; Ortúzar and Willumsen, 2011). Nevertheless, this criterion is often overlooked in the design of spatial divisions. The majority of transport models use administrative spatial delineations for data availability reasons. These delineations are often designed for statistical data collection purposes and hardly take into consideration intrazonal trips. Nevertheless, the minimization criterion should be considered when administrative spatial delineations are aggregated to construct new ones. Aggregated spatial designs should, as far as possible, minimize the rate of intrazonal trips. There remains the question of the right spatial aggregation level. This question has no straightforward answer. Transport modelers are often faced with a trade-off involving modeling details, accuracy, and development costs. In this context, the existence of the threshold effects is of interest.

Traffic assignment outcomes converge beyond a certain level of resolution or a number of zones. This threshold acknowledges the existence of a minimal number of zones that ensures a stable convergence of traffic assignment results. The inclusion of further spatial details beyond this threshold has marginal contribution given the corresponding costs. The existence of such a convergence threshold can help determine the appropriate spatial level of detail and the convenient trade-off between modeling resolution, development costs, and modeling accuracy. To do so, one does not need to re-apply the overall research methodology described here, but only to determine the minimal number of zones that guarantees a stable rate of intrazonal trips as demonstrated in chapter 4 .

Results pertaining to low road hierarchies, i.e. local streets, are found to be too sensitive towards the spatial aggregation bias. This network category is impacted by both the omission of intrazonal 
trips and the rerouting problem. Both these effects often occur at the vicinity of connection nodes. Given their functional use in transportation models, the extent of modeling errors induced by these effects can be relatively high ${ }^{4}$. Any finding drawn from this network is likely to be inaccurate and unreliable. This is a common wisdom in strategic models (Ortúzar and Willumsen, 2011). Nevertheless, with increasing availability of detailed data and computation capacities, a detailed representation and description of low road hierarchies might tempt academics and practitioners into inferring results from these networks and using them for policy instruction purposes. In this context, any conclusion is likely to be a mere and misleading illusion. This finding is of great interest to the design of transport models and to the choice of the right spatial resolution of the transportation network: the lowest road hierarchy is often the most impacted one. Therefore, one should always include a road category beyond the category intended for analysis. If a model is designed to study a road category $n$, this model should also include links of lower category $n+1$ to account for spatial aggregation problems. This recommendation has already been pointed out by Bovy and Jansen (1983).

\subsubsection{The spatial aggregation problem in traffic assignment mod- els}

\subsubsection{Thesis contribution}

Standard transport models often rely on a zonal description of space. This description induces several modeling errors known as the spatial aggregation problem. The omission of intrazonal trips in standard transport models is one consequence of this problem. The impact of this bias on assignment outcomes has been proven to be detrimental. To get rid of this bias or to reduce its magnitude, one solution is to re-integrate intrazonal trips in traffic assignment models. To this end, one needs to either disaggregate intrazonal travel demand between different subzones by using demand-side or mix methods or to include the impact of intrazonal flows in the definition of the transport network as suggested by supply-side methods. Findings suggest that the demand-side approach outperforms the supply-side one. The contribution of translating the impact of ignoring intrazonal trips in the definition of the transport network is limited if not inconclusive. This method fails, by design, to reproduce link flows. At the other hand, demand-side and mix methods are found to significantly improve modeling accuracy by accounting more explicitly for intrazonal demand in comparison with the standard traffic assignment model.

The contribution of demand-side-based assignment strategies is significant even when ad hoc methods are used to distribute intrazonal demand. When using a uniform distribution, traffic assignment outcomes are found to be less subject to the free-flow bias than with the standard assignment.

\footnotetext{
${ }^{4}$ In the case of link flows, local streets are likely to convey few car trips in average. In this case, modeling errors can have a highly relative impact.
} 
This is true for all road categories, from highway roads to local streets. Nevertheless, the uniform distribution induces an artificial increase in total Vehicle-Kilometers of Travel (VKT). This bias can be corrected for, or at least controlled for, if more convenient distribution methods are used instead of the uniform one. Nonetheless, despite the inclusion of intrazonal trips in traffic assignment, assignment results are still subject to the spatial aggregation problem and particularly to the rerouting problem. The inclusion of intrazonal trips in traffic assignment models is necessary but not sufficient to address the problem at hand.

The rerouting problem stems from the definition of centroid connectors. An arbitrary choice of connection nodes induces an arbitrary impact on route choice modeling and ultimately on link flows and traffic assignment outcomes (Manout and Bonnel, 2018; Sean Qian and Zhang, 2012). This problem has rarely been discussed in literature and has often been overlooked. Yet, the impact of connection nodes on traffic assignment outcomes is not marginal. In our case study, the rerouting problem is found to contribute to the free-flow bias by rerouting travel demand from congested routes to uncongested ones using centroid connectors. At certain levels of aggregation, the extent of this bias can outweigh that of the omission of intrazonal trips. One solution to reduce the impact of rerouting is to assign a volume-delay function and a finite capacity to centroid connectors. This straightforward solution does not address the rerouting problem but only reduces it extent. Another solution is to define centroid connectors using more accurate definitions instead of the standard one.

\subsubsection{Recommendations}

Two noteworthy recommendations are to be highlighted from the study of spatial aggregation in traffic assignment models:

1. One should include intrazonal demand in traffic assignment using demand-side methods.

2. A special attention should be paid to the definition of centroid connectors, especially in coarse models, to reduce the impact of the rerouting problem.

In traffic assignment, the inclusion of intrazonal demand using demand-side approaches has a significant contribution to modeling results. This inclusion improves the accuracy and reliability of assignment outcomes with comparison to the standard assignment strategy. This recommendation is especially of interest to coarse transport models where the rate of ignored intrazonal trips is more likely to be high. In these models, the inclusion of intrazonal trips using demand-side methods can mitigate the omission bias by reducing the free-flow effect. To this end, one needs to define an appropriate method to distribute intrazonal demand between sub-zones. In the current research, only the uniform distribution is tested. In this regard, findings suggest that the contribution of this straightforward method is significant. Nevertheless, this distribution method is also a source of bias that causes fictitious increase in average traveled distance. A special attention should therefore be put in the definition of the distribution method in demand-side-based assignment strategies. 
Despite the inclusion of intrazonal trips in traffic assignment, modeling results are likely to be still biased by the definition of centroid connectors. This bias is more difficult to get rid of since it is inherent to the design of conventional transport models that use zones to model space. In this regard, it is advisable to use a definition of connectors that is more robust towards spatial aggregation than the standard one. Such definition, should be as independent as possible from the spatial design by relying more on intrazonal data rather than on the position of zone centroids. Furthermore, findings suggest that setting a finite capacity and a volume-delay function on centroid connectors can reduce the extent of the rerouting bias.

\subsubsection{General contribution}

In this $\mathrm{PhD}$, we have set out to enhance the practicality of the standard LUTI modeling framework through the study of the spatial aggregation problem in traffic assignment models. To this end, two new modeling methods are suggested: a new model to define transit connectors and a new assignment strategy to assign intrazonal trips. These methods have proven to be useful by improving the quality, accuracy, and reliability of modeling outcomes. In this regard, modeling errors induced by the use of an aggregate spatial description are reduced. Moreover, a special emphasis has been put on the replicability, affordability, and responsiveness of these methods. As a consequence, some of these methods are currently under study in different spatial contexts for policy instruction purposes. Nevertheless, as any research work, this $\mathrm{PhD}$ is subject to limitations and shortcomings. Some of these limitations are due to the assumptions and methodological choices made by the author, other shortcomings are primarily driven by time constraints pertaining to this 40 months $\mathrm{PhD}$.

\subsection{Shortcomings and future work}

\subsubsection{Incomplete work}

In chapter 3, a new modeling method of transit connectors is suggested. This method is designed to overcome main shortcomings of the standard definition of centroid connectors. This new definition relies on intrazonal data instead of the geographic position of zone centroids. By doing so, the new definition of transit connectors is assumed to: (1) deliver more accurate results; (2) be more robust towards the spatial aggregation bias. In chapter 3, only the contribution to the accuracy of modeling outcomes is demonstrated. The capacity of this method to be robust towards the spatial aggregation bias is yet to be proved. A pending question is still to answer: What is the contribution of the new definition of transit connectors to address the spatial aggregation problem in transit modeling? In this regard, one can adopt a similar uncertainty modeling framework to that of chapters 4 and 5 to assess the impact of spatial aggregation on transit modeling and particularly 
the contribution of the new definition to reduce this impact. In this case, a special attention should be paid to intrazonal demand omission even if intrazonal transit demand is less likely to be impactful as in the case of traffic models. Conversely to traffic assignment models, transit models put less emphasis on the study of network congestion conditions. In this regard, the omission of intrazonal transit demand is relatively marginal.

In chapters 2 and 3, a special focus has been put on the definition of transit connectors. Car connectors have been neglected and their impact considered less detrimental than that of transit connectors. Findings from subsequent chapters 4 and 5 suggest, however, that the definition of car connectors has also a major impact on main traffic assignment results. The rerouting problem is one of these detrimental effects. This problem pertains to the choice of connection nodes and their impact on car flows and route choice. The extent of this impact can be potentially reduced using a convenient modeling method as the one suggested in chapter 3. This method puts a special emphasis on the selection of connection nodes and the computation of travel times. For lack of time, we could not apply this new definition to car connectors and appraise its contribution to address spatial aggregation problems in traffic assignment. This is a clear shortcoming of our research that can be addressed properly in future work by adapting the new definition of transit connectors to car connectors.

\subsubsection{Spatial aggregation, detailed data, and microsimulation}

In order to implement the new definition of centroid connectors or to assign intrazonal trips using new assignment strategies, one needs intrazonal data. Despite the fact that detailed data is becoming increasingly available, the use of this information is partly in contradiction with the practicality objectives sought after in this research. Models relying on detailed data are more likely to be difficult to replicate in different spatial contexts where these data are not available in comparison with models that use ubiquitous data. The same goes for development and maintenance costs that are likely to be higher when using more detailed data. This questions one of the main assumptions of the current research: standard LUTI models that rely on aggregate data are an appropriate modeling framework to develop practical models. In this regard, one can ask: Why bother using the standard modeling framework instead of the microsimulation framework if using detailed data?

In fact, this $\mathrm{PhD}$ is undertaken to address some of the flaws of conventional models. These flaws are not present or are less detrimental in other modeling frameworks like microsimulation. It is therefore legitimate to ask the above question and to justify the undergone efforts, especially, in view of the recent advances made in the microsimulation theory, data availability, and computation capacities. Models like agent-based or activity-based models address to some extent the above stated aggregation problem. However, microsimulation models are also subject to bias, and especially to uncertainty when modeling individual choices. At one hand, microsimulation data are not always available, and 
if so, they are prone to errors in measurement, especially in predictive situations. At the other hand, standard models alleviate measurement errors by using statistically consolidated/aggregated data which comes at a price of specification errors (Ay et al., 2017). A trade-off between these two types of errors: measurement and specification errors needs to be addressed in order to answer the above question (Alonso, 1968). Meanwhile, much of the current debate on the subject is either centered around the Cost/Data argument: microsimulation models are costly and require various detailed datasets that are context-specific or on the Practicality argument: standard models are more practical and are widely used by practitioners than microsimulation models. To these arguments, research should also consider the previous error trade-off in order to determine under which conditions specification errors overtake measurement errors and vice versa. The answer to these questions is not straightforward and is dependent on modeling objectives. Findings from the current $\mathrm{PhD}$ provide some insights into these research questions.

\subsubsection{Global contribution}

A major limitation of this research is the absence of a global assessment at the integrated LUTI modeling level: the impact of spatial aggregation and the contribution of new modeling strategies are only studied within the assignment framework. Nevertheless, assignment models take part of a global and systemic modeling framework where different sub-models interact and various feedback loops exist. Given this systemic architecture, any bias in any sub-model is likely to have repercussions on other sub-models. In the case of assignment, the spatial aggregation bias is proven to have an impact on travel times which are essential to modeling trips distribution, modal split, residential location/relocation, firms' location/relocation, and urban prices formation. The extent of this bias and its intricacies with other modeling errors are still to be explored and assessed at the LUTI modeling level of analysis. In this case, modeling errors can be either reduced or amplified given the existing non-linearities in LUTI models (Capelle et al., 2015; Pradhan and Kockelman, 2002; Ševčíková et al., 2007). In this regard, the reader can refer to the work of Jones (2016) for a study of spatial issues in LUTI models.

The same aforementioned criticism can be addressed to the new suggested modeling strategies: the contribution of these methods to the overall modeling quality is yet to be demonstrated and appraised at a global level. The contribution of the new definition of transit connectors is proven to improve main transit modeling outcomes; nevertheless, the contribution of this method to the accuracy of the overall LUTI model is not demonstrated. The same goes for the new assignment strategy of intrazonal trips. Further research is needed to unravel these questions in order to draw more global conclusions even if partial conclusions from this research are insightful. 


\subsubsection{Consistency between space and network}

In this research, a limited attention has been paid to the spatial consistency problem. This problem arises whenever inconsistent spatial resolutions are used to model different but related spatial phenomena. In traffic assignment models for instance, consistency between the spatial resolution of transportation networks and zones is required (Bovy and Jansen, 1983; Jeon et al., 2012). Nevertheless, network and spatial modeling are often studied separately (Connors and Watling, 2014). Within the LUTI framework where different spatial models interact, spatial inconsistencies may be a serious source of bias or inefficiencies. Modeling errors induced by coarse models are likely to spill over into detailed models and to counteract their accuracy. In this regard, relying on a consistent spatial resolution in the overall modeling chain is highly recommended. There remains the question of the appropriate spatial resolution that ensures such a consistency. The answer to this question is of interest to the definition of practical LUTI models and it has been let out of this research scope.

Finally, one of the objectives of the study of the spatial aggregation problem in LUTI models aims at enhancing the spatial replicability of these decision-aid tools. By spatial replicability we mean the ease with which a model can be applied in a useful way to different urban contexts ${ }^{5}$. The spatial replicability condition is vital to any operational LUTI model since it fosters a wide dissemination of these tools (popularization), allows for scale economies in development costs (budget savings), and above all, ensures a validation of these models in different contexts. Consequently, enhancing the spatial replicability of LUTI models is appealing. Findings from this $\mathrm{PhD}$ can improve the spatial replicability of transport models, in particular, and LUTI models in general. In fact, the study of the spatial aggregation problem is a step towards understanding the intricacies between space and models. By reducing the spatial aggregation bias, models are deemed to produce useful and reliable outcomes in different spatial contexts. Furthermore, the development of replicable, affordable, and responsive models is in line with the replicability objective.

\footnotetext{
${ }^{5}$ We prefer spatial replicability over spatial transferability since this latter expression is often used to describe the spatial transferability of parameter values from one context to another.
} 


\section{References}

Abdulaal, M., LeBlanc, L.J., 1979. Continuous equilibrium network design models. Transportation Research Part B: Methodological 13, 19-32. https://doi.org/10.1016/0191-2615(79)90004-3

Abrantes, P.A., Wardman, M.R., 2011. Meta-analysis of UK values of travel time: An update. Transportation Research Part A: Policy and Practice 45, 1-17. https://doi.org/10.1016/j.tra.2010. 08.003

Agence d'Urbanisme de Lyon, 2016. EMD 2015.

Agence d'Urbanisme de Lyon, 2006. EMD 2006.

Aigner, D.J., Goldfeld, S.M., 1974. Estimation and Prediction from Aggregate Data when Aggregates are Measured More Accurately than Their Components. Econometrica 42, 113-134. https://doi. org/10.2307/1913689

Alonso, W., 1968. Predicting best with imperfect data. Journal of the American institute of planners $34,248-255$.

Alonso, W., 1964. Location and land use. Toward a general theory of land rent. Location and land use. Toward a general theory of land rent.

Alshalalfah, B., Shalaby, A.S., 2007. Case study: Relationship of walk access distance to transit with service, travel, and personal characteristics. Journal of urban planning and development 133, $114-118$.

Amrhein, C.G., 1995. Searching for the elusive aggregation effect: Evidence from statistical simulations. Environment and Planning A 27, 105-119. https://doi.org/10.1068/a270105

Anas, A., Liu, Y., 2007. A regional economy, land use, and transportation model (relu-tran): Formulation, algorithm design, and testing. Journal of Regional Science 47, 415-455.

Anselin, L., 2013. Spatial econometrics: Methods and models. Springer Science \& Business Media.

Atherton, T.J., Ben-Akiva, M.E., 1976. Transferability and Updating of Disaggregate Travel Demand Models 7. 
Ay, J.-S., Chakir, R., Le Gallo, J., 2017. Aggregated versus individual land-use models: Modeling spatial autocorrelation to increase predictive accuracy. Environmental Modeling \& Assessment 22, 129-145.

Baaj, M.H., Mahmassani, H.S., 1995. Hybrid route generation heuristic algorithm for the design of transit networks. Transportation Research Part C: Emerging Technologies 3, 31-50. https: //doi.org/10.1016/0968-090X(94)00011-S

Baass, 1981. Travel demand forecasting and data considerations. National Academy of Sciences, Washington, D.C.

Badoe, D.A., Miller, E.J., 2000. Transportationland-use interaction: Empirical findings in North America, and their implications for modeling. Transportation Research Part D: Transport and Environment 5, 235-263.

Bain, R., 2009. Error and optimism bias in toll road traffic forecasts. Transportation 36, 469-482.

Bar-Gera, H., Hellman, F., Patriksson, M., 2013. Computational precision of traffic equilibria sensitivities in automatic network design and road pricing. Transportation Research Part B: Methodological 57, 485-500. https://doi.org/10.1016/j.trb.2013.08.018

Batty, M., 1976. Urban modelling: Algorithms calibrations, predictions, Cambridge urban and architectural studies. Cambridge University Press, Cambridge ; New York.

Batty, M., 1974. Spatial Entropy. Geographical Analysis 6, 1-31. https://doi.org/DOI:/\%002010. 1111/j.1538-4632.1974.tb01014.x

Batty, M., Sikdar, P.K., 1982a. Spatial aggregation in gravity models. 1. An information-theoretic framework. Environment and Planning A 14, 377-405. https://doi.org/10.1068/a140377

Batty, M., Sikdar, P.K., 1982b. Spatial aggregation in gravity models: 2. One-dimensional population density models. Environment and Planning A 14, 525-553. https://doi.org/10.1068/a140525

Batty, M., Sikdar, P.K., 1982c. Spatial aggregation in gravity models: 3. Two-dimensional trip distribution and location models. Environment and Planning A 14, 629-658. https://doi.org/10. $1068 / \mathrm{a} 140629$

Batty, M., Sikdar, P.K., 1982d. Spatial aggregation in gravity models: 4. Generalisations and large-scale applications. Environment and Planning A 14, 795-822. https://doi.org/10.1068/a140795

Baumol, W.J., Oates, W.E., 1988. The theory of environmental policy, 2nd ed. ed. Cambridge University Press, Cambridge [Cambridgeshire] ; New York.

Beckmann, M., McGuire, C.B., Winsten, C., 1956. Studies in the Economics of Transportation. Yale University Press and Oxford University Presss.

Ben-Akiva, M., Bolduc, D., 1987. Approaches to Model Transferability and Updating: The Combined 
Transfer Estimator. Transportation Research Record 7.

Ben-Akiva, M.E., Lerman, S.R., 1985. Discrete choice analysis: Theory and application to travel demand. MIT press.

Ben-Akiva, M., Lerman, S.R., 1979. Disaggregate travel and mobility choice models and measures of accessibility. Behavioural travel modelling 654-679.

Bhatta, B.P., Larsen, O.I., 2011. Are intrazonal trips ignorable? Transport Policy 18, 13-22. https://doi.org/10.1016/j.tranpol.2010.04.004

Binetti, M., Ciani, E., 2002. Effects of traffic analysis zones design on transportation models, in: Proceedings of the 9th Meeting of the Euro Working Group on Transportation, Bari, Italy.

Bonnel, P., 2004. Prévoir la demande de transport. Presses de l'École Nationale des Ponts et Chaussées, Paris.

Bovy, P.H.L., Jansen, G.R.M., 1983. Network Aggregation Effects upon Equilibrium Assignment Outcomes: An Empirical Investigation. Transportation Science 17, 240.

Branston, D., 1976. Link capacity functions: A review. Transportation Research 10, 223-236. https://doi.org/10.1016/0041-1647(76)90055-1

Briant, A., Combes, P.-P., Lafourcade, M., 2010. Dots to boxes: Do the size and shape of spatial units jeopardize economic geography estimations? Journal of Urban Economics 67, 287-302. https://doi.org/10.1016/j.jue.2009.09.014

Brons, M., Givoni, M., Rietveld, P., 2009. Access to railway stations and its potential in increasing rail use. Transportation Research Part A: Policy and Practice 43, 136-149. https://doi.org/10.1016/ j.tra.2008.08.002

Brown, M.B., Forsythe, A.B., 1974a. Robust tests for the equality of variances. Journal of the American Statistical Association 69, 364-367.

Brown, M.B., Forsythe, A.B., 1974b. The small sample behavior of some statistics which test the equality of several means. Technometrics 16, 129-132.

Bureau of Public Roads, 1964. Traffic assignment manual. U.S. Department of Commerce, Urban Planning Division, Washington D.C.

Cambridge Systematics, I., Consult, A., 2007. A Recommended Approach to Delineating Traffic Analysis Zones in Florida.

Campbell, S., 1996. Green cities, growing cities, just cities?: Urban planning and the contradictions of sustainable development. Journal of the American Planning Association 62, 296-312.

Capelle, T., Sturm, P., Vidard, A., 2015. Formulating LUTI calibration as an optimisation problem: Example of tranus shadow price estimation. Procedia Engineering 115, 12-20. 
Cardozo, O.D., García-Palomares, J.C., Gutiérrez, J., 2012. Application of geographically weighted regression to the direct forecasting of transit ridership at station-level. Applied Geography 34, 548-558. https://doi.org/10.1016/j.apgeog.2012.01.005

Cervero, R., Kockelman, K., 1997. Travel demand and the 3Ds: Density, diversity, and design. Transportation Research Part D: Transport and Environment 2, 199-219.

Cervero, R., Seskin, S., 1995. An evaluation of the relationships between transit and urban form. TCRP Research Results Digest.

Chan, Y., 1976. A method to simplify network representation in transportation planning. Transportation Research 10, 179-191. https://doi.org/10.1016/0041-1647(76)90073-3

Chang, K.-T., Khatib, Z., Ou, Y., 2002. Effects of Zoning Structure and Network Detail on Traffic Demand Modeling. Environment and Planning B: Planning and Design 29, 37-52. https: //doi.org/10.1068/b2742

Chapleau, R., de Cea, J., 1983. La Perception de l'offre par les usagers du transport en commun sous la perspective d'un modèle d'affectation. Routes et Transports.

Chatzis, K., 2013. Une histoire comparée de la modélisation des déplacements urbains en France et en Amérique du Nord (Etats-Unis et Canada) sur la longue durée (1950 à nos jours).

Chowdhury, S., Zhai, K., Khan, A., 2016. The Effects of Access and Accessibility on Public Transport Users' Attitudes. Journal of Public Transportation 19, 97-113. https://doi.org/10.5038/23750901.19.1.7

Christaller, W., 1966. (1933) central places in southern germany. Prentice Hall.

Clark, W.A.V., Avery, K.L., 1975. The Effects of Data Aggregation in Statistical Analysis. Geographical Analysis 8, 428-438. https://doi.org/10.1111/j.1538-4632.1976.tb00549.x

Commissariat Général à la Stratégie et à la Prospective, 2013. L'évaluation socioéconomique des investissements publics. Tome 1.

Connors, R.D., Watling, D.P., 2014. Assessing the Demand Vulnerability of Equilibrium Traffic Networks via Network Aggregation. Networks and Spatial Economics. https://doi.org/10.1007/ s11067-014-9251-9

Connors, R.D., Watling, D.P., 2008. Aggregation of Transport Networks Using Sensitivity Analysis. Association for european transport.

Cramer, J.S., 1964. Efficient Grouping, Regression and Correlation in Engel Curve Analysis. Journal of the American Statistical Association 59, 233-250.

Crevo, 1991. Impacts of zonal reconfigurations on travel demand forecasts. Transportation Research Record. 
Daganzo, C.F., 1980a. Network representation, continuum approximations and a solution to the spatial aggregation problem of traffic assignment. Transportation Research Part B: Methodological 14, 229-239. https://doi.org/10.1016/0191-2615(80)90002-8

Daganzo, C.F., 1980b. An equilibrium algorithm for the spatial aggregation problem of traffic assignment. Transportation Research Part B: Methodological 14, 221-228. https://doi.org/10.1016/ 0191-2615(80)90001-6

Daniels, R., Mulley, C., 2013. Explaining walking distance to public transport: The dominance of public transport supply. Journal of Transport and Land Use 6, 5-20.

de la Barra, T., 1999. Mathematical description of TRANUS. Technical report, Modelistica, Caracas, Venezuela.

DeCorla-Souza, P., Grubb, N.J., 1991. Network focusing: A tool for quick-response subarea analysis. ITE Journal 61.

Delbosc, A., Currie, G., 2011. Exploring the relative influences of transport disadvantage and social exclusion on well-being. Transport Policy 18, 555-562.

Delons, J., Coulombel, N., Leurent, F., 2008. PIRANDELLO an integrated transport and land-use model for the Paris area.

Denant-Boèmont, L., 1994. Social cost-benefit analysis and irreversibility of technical choices in transit. HAL.

Dijkstra, E.W., 1959. A note on two problems in connexion with graphs. Numerische mathematik 1, 269-271.

Dill, J., 2003. Transit use and proximity to rail: Results from large employment sites in the San Francisco, California, bay area. Transportation Research Record: Journal of the Transportation Research Board 1835, 19-24.

Ding, C., 1998. The GIS-based human-interactive TAZ design algorithm: Examining the impacts of data aggregation on transportation-planning analysis. Environment and Planning B: Planning and Design 25, 601-616. https://doi.org/10.1068/b250601

Ding, C., 1994. Impact analysis of spatial data aggregation on transportation forecasted demand: A GIS approach. URISA Proceedings URISA 1460, 362-375.

Dunn, D.M., Williams, W.H., DeChaine, T.L., 1976. Aggregate Versus Subaggregate Models in Local Area Forecasting. Journal of the American Statistical Association 71, 68-71. https: //doi.org/10.2307/2285732

Dupuy, G., 1975. Une technique de planification au service de l'automobile : les modèles de trafic urbain. 
Eash, R.W., Chon, K.S., Lee, Y.J., Boyce, D.E., 1988. Equilibrium Traffic Assignment on an Aggregated Highway Network for Sketch Planning. Transportation Research Record 8.

Echenique, M.H., Flowerdew, A.D., Hunt, J.D., Mayo, T.R., Skidmore, I.J., Simmonds, D.C., 1990. The meplan models of bilbao, leeds and dortmund. Transport Reviews 10, 309-322.

El-Geneidy, A., Grimsrud, M., Wasfi, R., Tétreault, P., Surprenant-Legault, J., 2014. New evidence on walking distances to transit stops: Identifying redundancies and gaps using variable service areas. Transportation 41, 193-210. https://doi.org/10.1007/s11116-013-9508-z

Emberger, G., Pfaffenbichler, P., Jaensirisak, S., Timms, P., 2008. "Ideal" decision-making processes for transport planning: A comparison between Europe and South East Asia. Transport Policy 15, 341-349. https://doi.org/10.1016/j.tranpol.2008.12.009

Enrique Fernández L., J., de Cea Ch., J., Malbran, R.H., 2008. Demand responsive urban public transport system design: Methodology and application. Transportation Research Part A: Policy and Practice 42, 951-972. https://doi.org/10.1016/j.tra.2007.12.008

Ewing, R., Cervero, R., 2001. Travel and the Built Environment: A Synthesis. Transportation Research Record 1780, 87-114. https://doi.org/10.3141/1780-10

Farhan, B., Murray, A.T., 2006. Distance decay and coverage in facility location planning. The Annals of Regional Science 40, 279-295. https://doi.org/10.1007/s00168-005-0041-7

Flyvbjerg, B., 2007. Megaproject policy and planning: problems, causes, cures = Megaprojekters politik og planlægning. Institut for Samfundsudvikling og Planlægning, Aalborg Universitet, Aalborg.

Flyvbjerg, B., 2005. Measuring inaccuracy in travel demand forecasting: Methodological considerations regarding ramp up and sampling. Transportation Research Part A: Policy and Practice 39, $522-530$.

Flyvbjerg, B., Skamris Holm, M.K., Buhl, S.L., 2005. How (in) accurate are demand forecasts in public works projects?: The case of transportation. Journal of the American planning association $71,131-146$.

Fotheringham, A.S., Wong, D.W.S., 1991. The modifiable areal unit problem in multivariate statistical analysis. Environment and Planning A 23, 1025-1044. https://doi.org/10.1068/a231025

Frank, L.D., Pivo, G., 1994. Impacts of mixed use and density on utilization of three modes of travel: Single-occupant vehicle, transit, and walking. Transportation research record 1466, 44-52.

Frank, M., Wolfe, P., 1956. An algorithm for quadratic programming. Naval Research Logistics Quarterly 3, 95-110. https://doi.org/10.1002/nav.3800030109

Friedrich, M., Galster, M., 2009. Methods for Generating Connectors in Transport Planning Models. Transportation Research Record: Journal of the Transportation Research Board 2132, 133-142. 
https://doi.org/10.3141/2132-15

Friedrich, M., Hofsaess, I., Wekeck, S., 2001. Timetable-Based Transit Assignment Using Branch and Bound Techniques. Transportation Research Record: Journal of the Transportation Research Board 1752, 100-107. https://doi.org/10.3141/1752-14

Friesz, T.L., 1985. Transportation network Equilibrium, Design and Aggregation: key developments and research opportunities. The automobile 7, 296.

Friesz, T.L., Cho, H.-J., Mehta, N.J., Tobin, R.L., Anandalingam, G., 1992. A Simulated Annealing Approach to the Network Design Problem with Variational Inequality Constraints. Transportation Science 26, 18-26. https://doi.org/10.1287/trsc.26.1.18

Fujita, M., Krugman, P.R., Venables, A., 1999. The spatial economy: Cities, regions and international trade. MIT Press, Cambridge, Mass.

Fujita, M., Thisse, J.-F., 2003. Economie des villes et de la localisation. De Boeck Supérieur.

Gehlke, C.E., Biehl, K., 1934. Certain Effects of Grouping Upon the Size of the Correlation Coefficient in Census Tract Material. Journal of the American Statistical Association 29, 169. https://doi.org/10.2307/2277827

Geurs, K.T., Krizek, K.J., Reggiani, A., 2012. Accessibility analysis and transport planning: Challenges for europe and north america. Edward Elgar Publishing.

Great Britain, Highways Agency, 1996. Design manual for roads and bridges. Vol. 12 a, Vol. 12 a, H.M.S.O., London.

Griffith, D.A., 1983. The boundary value problem in spatial statistical analysis. Journal of regional science $23,377-387$.

Guo, J.Y., Bhat, C.R., 2004. Modifiable Areal Units: A Problem or a Matter of Perception in the Context of Residential Location Choice Modeling?

Gutiérrez, J., Cardozo, O.D., García-Palomares, J.C., 2011. Transit ridership forecasting at station level: An approach based on distance-decay weighted regression. Journal of Transport Geography 19, 1081-1092. https://doi.org/10.1016/j.jtrangeo.2011.05.004

Haghani, A.E., Daskin, M.S., 1986. Aggregation effects on the network design problem. Journal of advanced transportation 20, 239-258.

Haghani, A.E., Daskin, M.S., 1983. Network design application of an extraction algorithm for network aggregation. Transportation Research Record.

Hannan, M.T., 1972. Approaches to the Aggregation Problem.

Hansen, W.G., 1959. How Accessibility Shapes Land Use. Journal of the American Institute of Planners 25, 73-76. https://doi.org/10.1080/01944365908978307 
Hardy, M.H., 2011. Simplified integrated transportation and land use modeling to support metropolitan planning decisions: An application and assessment ( $\mathrm{PhD}$ thesis).

Higgins, C., Ferguson, M., Kanaroglou, P., 2014. Light rail and land use change: Rail transit's role in reshaping and revitalizing cities. Journal of Public Transportation 17, 5.

Hoover, E.M., 1937. Location theory and the shoe leather industries. Harvard University Press.

Horni, A., Nagel, K., Axhausen, K.W., 2016. The multi-agent transport simulation matsim. Ubiquity Press London:

Horowitz, A.J., 2001. Computational issues in increasing spatial precision of traffic assignments. Transportation Research Record: Journal of the Transportation Research Board 1777, 68-74.

Hsiao, S., Lu, J., Sterling, J., Weatherford, M., 1997. Use of geographic information system for analysis of transit pedestrian access. Transportation Research Record: Journal of the Transportation Research Board 1604, 50-59.

Iacono, M., Krizek, K., El-Geneidy, A.M., 2008. Access to destinations: How close is close enough? Estimating accurate distance decay functions for multiple modes and different purposes.

Ibeas, Á., dell'Olio, L., Alonso, B., Sainz, O., 2010. Optimizing bus stop spacing in urban areas. Transportation Research Part E: Logistics and Transportation Review 46, 446-458. https: //doi.org/10.1016/j.tre.2009.11.001

IGN, 2008. Descriptif du contenu de la BD TOPO Version 2.

Ijiri, Y., 1971. Fundamental Queries in Aggregation Theory. Journal of the American Statistical Association 66, 766-782.

Inoue, S.-i., Maruyama, T., 2012. Computational experience on advanced algorithms for user equilibrium traffic assignment problem and its convergence error. Procedia-Social and Behavioral Sciences 43, 445-456.

Insee, 2017. Découpage infracommunal en IRIS.

Insee, 2013. Recensement 2013 : Résultats sur un territoire, bases de données et fichiers détail.

Insee, 2011. Description de la structure de la base de données SIRENE et des fichiers de mise à jour.

Jeon, J.-H., Kho, S.-Y., Park, J.J., Kim, D.-K., 2012. Effects of spatial aggregation level on an urban transportation planning model. KSCE Journal of Civil Engineering 16, 835-844. https: //doi.org/10.1007/s12205-012-1400-4

Jones, J., 2016. Spatial bias in luti models (PhD thesis). Doctoral dissertation, Presse Universitaire de Louvain, Belgique.

Kordi, M., Kaiser, C., Fotheringham, A.S., 2012. A possible solution for the centroid-to-centroid and intra-zonal trip length problems, in: International Conference on Geographic Information Science, 
Avignon.

Krugman, P.R., 1997. Development, geography, and economic theory. MIT press.

Kuby, M., Barranda, A., Upchurch, C., 2004. Factors influencing light-rail station boardings in the United States. Transportation Research Part A: Policy and Practice 38, 223-247. https: //doi.org/10.1016/j.tra.2003.10.006

Le Gallo, J., others, 2000. Econométrie spatiale (1, autocorrélation spatiale). LATEC.

Lee Jr, D.B., 1973. Requiem for large-scale models. Journal of the American Institute of planners $39,163-178$.

Leontief, W.W., 1986. Input-output economics. Oxford University Press.

Leurent, F., Benezech, V., Samadzad, M., 2011. A stochastic model of trip end disaggregation in traffic assignment to a transportation network. Procedia - Social and Behavioral Sciences, The state of the art in the european quantitative oriented transportation and logistics research 14th euro working group on transportation \& 26th mini euro conference \& 1st european scientific conference on air transport 20, 485-494. https://doi.org/10.1016/j.sbspro.2011.08.055

Lowry, I.S., 1964. A Model of Metropolis RM-4035-RC. Rand Corporation, Santa Monica, CA. Luathep, P., Sumalee, A., Lam, W.H., Li, Z.-C., Lo, H.K., 2011. Global optimization method for mixed transportation network design problem: A mixed-integer linear programming approach. Transportation Research Part B: Methodological 45, 808-827. https://doi.org/10.1016/j.trb.2011. 02.002

Malinvaud, E., 1993. A framework for aggregation theories. Ricerche Economiche 47, 107-135. https://doi.org/10.1016/0035-5054(93)90015-U

Malinvaud, E., 1956. L'agrégation dans les modèles économiques. Cahiers du Séminaire d'Économétrie 69. https://doi.org/10.2307/20075377

Mann, W.W., 2002. B-node model: New subarea traffic assignment model \& application, in: Eighth TRB Conference on the Application of Transportation Planning Methods.

Manout, O., 2014. Codification des connecteurs de zones pour les transports en commun. University of Lyon - ENTPE.

Manout, O., Bonnel, P., 2018. The impact of ignoring intrazonal trips in assignment models: A stochastic approach. Transportation. https://doi.org/10.1007/s11116-018-9951-y

Manout, O., Bonnel, P., Bouzouina, L., 2018. Transit accessibility: A new definition of transit connectors. Transportation Research Part A: Policy and Practice 113, 88-100. https://doi.org/10. 1016/j.tra.2018.03.028

Marshall, A., 1895. Principles of economics. 
Martínez, L.M., Viegas, J.M., Silva, E.A., 2009. A traffic analysis zone definition: A new methodology and algorithm. Transportation 36, 581-599. https://doi.org/10.1007/s11116-009-9214-z

Masser, I., Brown, P.J.B., 1975. Hierarchical Aggregation Procedures for Interaction Data. Environment and Planning A 7, 509-523. https://doi.org/10.1068/a070509

May, A.D., Karlstrom, A., Marler, N., Matthews, B., Minken, H., Monzon, A., Page, M., Pfaffenbichler, P., Shepherd, S., 2003. Developing Sustainable Urban Land Use and Transport Strategies-A Decision Makers' Guidebook. Institute for Transport Studies, University of Leeds, Leeds.

McFadden, D., 1978. Modelling the choice of residential location. Institute of Transportation Studies, University of California.

Meng, Q., Yang, H., Bell, M., 2001. An equivalent continuously differentiable model and a locally convergent algorithm for the continuous network design problem. Transportation Research Part B: Methodological 35, 83-105. https://doi.org/10.1016/S0191-2615(00)00016-3

Métropole de Lyon, 2015. Horaires théoriques du réseau TCL. Data Grand Lyon.

Miller, E.J., 2018. The case for microsimulation frameworks for integrated urban models. Journal of Transport and Land Use 11.

Miller, H.J., 1999. Potential Contributions of Spatial Analysis to Geographic Information Systems for Transportation (GIS-T). Geographical Analysis 31, 373-399. https://doi.org/10.1111/j.15384632.1999.tb00991.x

Moeckel, R., Donnelly, R., 2009. Simulation of intrazonal traffic flows: The end of lost trips.

Nicolas, J.P., Bonnel, P., Cabrera, J., Godinot, C., Homocianu, M., Routhier, J.L., Toilier, F., Zuccarello, P., 2009. SImuler les MoBilités pour une Agglomération Durable. Rapport final du projet Simbad, Rapport de recherche du Laboratoire d'Économie des Transports Pour le compte de la DRI (MEEDDAT) et de l'ADEME dans le cadre du groupe.

North, D.C., 1955. Location theory and regional economic growth. Journal of political economy 63, $243-258$.

Openshaw, S., 1983. Modifiable Areal Unit Problem. Geo Books, Norwich [Norfolk].

Openshaw, S., 1979. A million or so correlation coefficients: Three experiments on the modifiable areal unit problem. Statistical applications in the spatial science 127-144.

Openshaw, S., 1978. An empirical study of some zone-design criteria. Environment and planning A 10, 781-794.

Openshaw, S., 1977a. A Geographical Solution to Scale and Aggregation Problems in RegionBuilding, Partitioning and Spatial Modelling. Transactions of the Institute of British Geographers 2, 459. https://doi.org/10.2307/622300 
Openshaw, S., 1977b. Optimal zoning systems for spatial interaction models. Environment and Planning A 9, 169-184.

Ortúzar, J. de D., Willumsen, L., 2011. Modelling transport, 4th ed. Wiley-Blackwell, Oxford.

O'Neill, W.A., 1991. Developing optimal transportation analysis zones using GIS, in: Proceedings of the 1991 Geographic Information Systems (GIS) for Transportation Symposium.

O'sullivan, A., 2007. Urban economics. McGraw-Hill/Irwin.

O'Sullivan, S., Morrall, J., 1996. Walking distances to and from light-rail transit stations. Transportation research record: journal of the transportation research board 1538, 19-26.

Parsons Brinckerhoff Quade \& Douglas, I., 1993. Building orientation a supplement to the pedestrian environment.

Patriksson, M., 2015. The traffic assignment problem: Models and methods. Courier Dover Publications.

Pigou, A., 2017. The Economics of Welfare. Routledge. https://doi.org/10.4324/9781351304368

Plaza, C.V., da Silva, A.N.R., 2015. Intrazonal Trip Distances: An Estimation Approach and Application to a Case Study, in: Transportation Research Board 94th Annual Meeting. Citeseer.

Pradhan, A., Kockelman, K., 2002. Uncertainty propagation in land use-transportation models, in: Proceedings of the 13th Euro Mini Conference on Handling Uncertainty in the Analysis of Traffic and Transportation Systems, Bari, Italy.

Quinet, E., 2010. La pratique de l'analyse coût-bénéfice dans les transports : Le cas de la France. OECD Publishing.

Redman, L., Friman, M., Gärling, T., Hartig, T., 2013. Quality attributes of public transport that attract car users: A research review. Transport Policy 25, 119-127. https://doi.org/10.1016/j. tranpol.2012.11.005

Reggiani, A., 2019. Accessibility, trade and locational behaviour. Routledge.

Robinson, W., 1950. Ecological correlations and the behavior of individuals. American Sociological Review 15.

Rodriguez-Bachiller, A., 1983. Errors in the Measurement of Spatial Distances between Discrete Regions. Environment and Planning A 15, 781-799. https://doi.org/10.1068/a150781

Rose, G., Daskin, M.S., Koppelman, F.S., 1988. An examination of convergence error in equilibrium traffic assignment models. Transportation Research Part B: Methodological 22, 261-274. https: //doi.org/10.1016/0191-2615(88)90003-3

Salling, K.B., Leleur, S., 2015. Accounting for the inaccuracies in demand forecasts and construction cost estimations in transport project evaluation. Transport Policy 38, 8-18. https://doi.org/10. 
1016/j.tranpol.2014.11.006

Saujot, M., De Lapparent, M., Arnaud, E., Prados, E., 2015. To make LUTI models operationnal tools for planning, in: International Conference on Computers in Urban Planning and Urban Management (CUPUM).

Schöbel, A., Hamacher, H.W., Liebers, A., Wagner, D., 2009. The continuous stop location problem in public transportation networks. Asia-Pacific Journal of Operational Research 26, 13-30.

Sean Qian, Z., Zhang, H., 2012. On centroid connectors in static traffic assignment: Their effects on flow patterns and how to optimize their selections. Transportation Research Part B: Methodological 46, 1489-1503. https://doi.org/10.1016/j.trb.2012.07.006

Sheffi, Y., 1984. Urban transportation networks: Equilibrium analysis with mathematical programming methods. Prentice-Hall, Englewood Cliffs, N.J.

Simini, F., González, M.C., Maritan, A., Barabási, A.-L., 2012. A universal model for mobility and migration patterns. Nature 484, 96-100. https://doi.org/10.1038/nature10856

Simmonds, D.C., 1999. The design of the DELTA land-use modelling package. Environment and Planning B 26, 665-684.

Simmonds, D., Echenique, M., Bates, J., Oosterhaven, J., 1999. Review of land-use/transport interaction models. Reports to The Standing Advisory Committee on Trunk Road Assessment, Department of the Environment, Transport and the Regions, London.

Skamris, M.K., Flyvbjerg, B., 1997. Inaccuracy of traffic forecasts and cost estimates on large transport projects. Transport Policy 4, 141-146. https://doi.org/10.1016/S0967-070X(97)00007-3

Smith, J., Blewitt, R., 2010. Traffic modelling guidelines: TfL traffic manager and network performance best practice version 3.0, London: Transport for London.

Snedecor, G.W.C., William, G., 1989. Statistical methods.

Southworth, F., 1995. A technical review of urban land use-transportation models as tools for evaluating vehicle travel reduction strategies.

Spiess, H., 1990. Technical Note-Conical Volume-Delay Functions. Transportation Science 24, 153-158. https://doi.org/10.1287/trsc.24.2.153

Spiess, H., Florian, M., 1989. Optimal strategies: A new assignment model for transit networks. Transportation Research Part B: Methodological 23, 83-102. https://doi.org/10.1016/0191-2615(89) 90034-9

Stewart Fotheringham, A., Rogerson, P.A., 1993. GIS and spatial analytical problems. International journal of geographical information systems 7, 3-19. https://doi.org/10.1080/02693799308901936 STIF, OMNIL, DRIEA, 2010. Résultats détaillées de l'Enquête Globale Transports 2010. 
Stouffer, S.A., 1940. Intervening Opportunities: A Theory Relating Mobility and Distance. American Sociological Review 5, 845-867. https://doi.org/10.2307/2084520

SYTRAL, 2018. Plan de déplacements urbains de l'Agglomération lyonnaise: 2017-2030. http: //www.sytral.fr/157-plan-deplacements-urbains.htm.

Ševčíková, H., Raftery, A.E., Waddell, P.A., 2007. Assessing uncertainty in urban simulations using bayesian melding. Transportation Research Part B: Methodological 41, 652-669.

Tabuchi, T., Thisse, J.-F., 2011. A new economic geography model of central places. Journal of Urban Economics 69, 240-252.

Tamblay, S., Galilea, P., Iglesias, P., Raveau, S., Muñoz, J.C., 2016. A zonal inference model based on observed smart-card transactions for Santiago de Chile. Transportation Research Part A: Policy and Practice 84, 44-54. https://doi.org/10.1016/j.tra.2015.10.007

Texas Transportation Institute, State Department of Highways and public transportation, 1988. Subarea analysis using TRANPLAN/NEDS (No. FHWA/TX-87/1110-4F).

Theil, H., 1954. Linear aggregation of economic relations.

Thomas, I., Jones, J., Caruso, G., Gerber, P., 2018. City delineation in European applications of LUTI models: Review and tests. Transport Reviews 38, 6-32. https://doi.org/10.1080/01441647. 2017.1295112

Thünen, J.H. von, 1826. Der isolierte staat in beziehung auf nationalökonomie und landwirtschaft. Gustav Fischer, Stuttgart (reprinted 1966).

Timmermans, H., 2003. The saga of integrated land use-transport modeling: How many more dreams before we wake up, in: Keynote Paper, Moving Through Nets: The Physical and Social Dimension of Travel, 10th International Conference on Travel Behaviour Research, Lucerna, Www. Ivt. Baug. Ethz. Ch/Allgemein/Pdf/Timmermans. Pdf.

Tobler, W.R., 1989. Frame independent spatial analysis. Accuracy of spatial databases 115-122.

Transportation Research Board, National Research Council, 2000. Highway capacity manual. Washington, D.C.

United Nations, D. of E., Social Affairs, P.D., 2018. World urbanization prospects: The 2018 revision, custom data acquired via website.

Venables, A., 2005. Anthony j. Venables, london school of economics and cepr.

Venigalla, M.M., Chatterjee, A., Bronzini, M.S., 1999. A specialized equilibrium assignment algorithm for air quality modeling. Transportation Research Part D: Transport and Environment 4, 29-44. https://doi.org/10.1016/S1361-9209(98)00022-4 
Waddell, P., 2011. Integrated land use and transportation planning and modelling: Addressing challenges in research and practice. Transport Reviews 31, 209-229.

Waddell, P., 2002. UrbanSim: Modeling urban development for land use, transportation, and environmental planning. Journal of the American Planning Association 68, 297-314.

Walker, L., 2014. Does size matter? The benefits of disaggregated zone systems in strategic transport models, in:.

Wang, S., Meng, Q., Yang, H., 2013. Global optimization methods for the discrete network design problem. Transportation Research Part B: Methodological 50, 42-60. https://doi.org/10.1016/j.trb. 2013.01.006

Wardman, M., 2001. A review of British evidence on time and service quality valuations. Transportation Research Part E: Logistics and Transportation Review 37, 107-128. https://doi.org/10. 1016/S1366-5545(00)00012-0

Wardrop, J.G., 1952. Some theoretical aspects of road traffic research. Proceedings of the Institution of Civil Engineers 1, 325-362. https://doi.org/10.1680/ipeds.1952.11259

WATS, 2008. Zone centroid and centroid connector placement.

Weber, A., 1929. (1909) theory of the location of industries. University of Chicago Press.

Wegener, M., 2011. From macro to microhow much micro is too much? Transport Reviews 31, $161-177$.

Wegener, M., 2004. Overview of land-use transport models. Handbook of transport geography and spatial systems $5,127-146$.

Wegener, M., 1998. Applied models of urban land use, transport and environment: State of the art and future developments, in: Network Infrastructure and the Urban Environment. Springer, pp. 245-267.

Wegener, M., Fuerst, F., 2004. Land-Use Transport Interaction: State of the Art. SSRN Electronic Journal. https://doi.org/10.2139/ssrn.1434678

Welch, B.L., 1947. The generalization of "student's" problem when several different population variances are involved. Biometrika 34, 28-35. https://doi.org/10.1093/biomet/34.1-2.28

Wilson, A.G., 1969. The use of entropy maximising models, in the theory of trip distribution, mode split and route split. Journal of Transport Economics and Policy 108-126.

Wong, D.W.S. (Ed.), 2004. The Modifiable Areal Unit Problem (MAUP). Springer Netherlands, Dordrecht.

World Bank, 2005. Demand forecasting errors (No. Transport Note No. TRN-12). 
You, J., Nedović-Budić, Z., Kim, T.J., 1998. A GIS-based traffic analysis zone design: Implementation and evaluation. Transportation Planning and Technology 21, 69-91. https://doi.org/10.1080/ 03081069708717602

Yule, G.U., Kendall, M., 1950. An introduction to the theory of statistics, charles griffin \& co. Ltd., London.

Zhao, F., Chow, L.-F., Li, M.-T., Ubaka, I., Gan, A., 2003. Forecasting transit walk accessibility: Regression model alternative to buffer method. Transportation Research Record: Journal of the Transportation Research Board 1835, 34-41. 


\section{Appendix}

\section{A Relative Difference and GEH indicators of Sioux Falls}

A.1 Zoning 2: zones 1, 3, 4, 11, 12, 13, 14, 23, 24

This zoning produces $12 \%$ of intrazonal trips. 
A.1.1 Method 2, approach 2: subdivision of zones using the original distribution of intrazonal demand

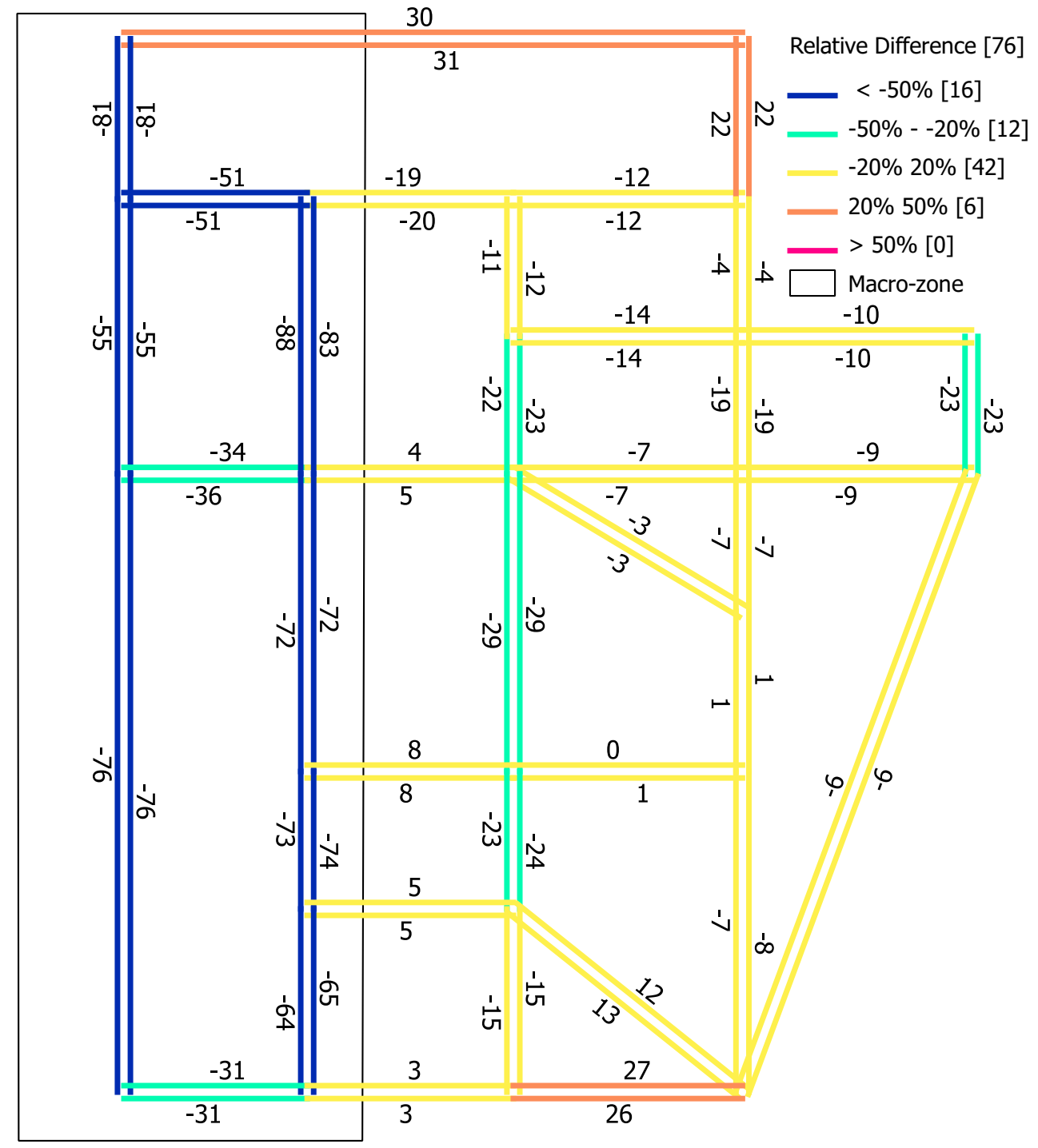

Figure 7.1: Zoning 2: the relative difference (RD) of link flows produced by method 2.2 on the Sioux Falls case study 


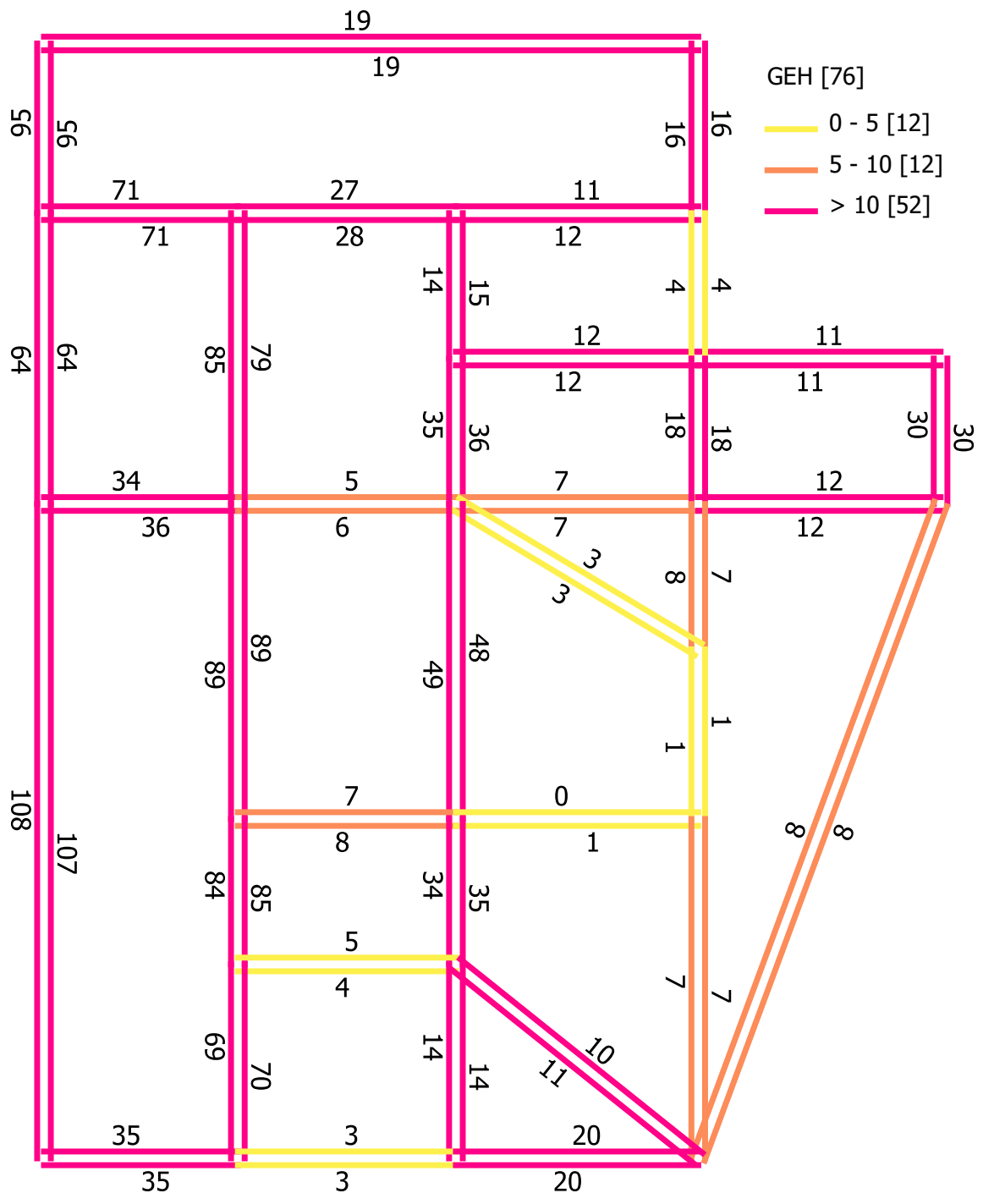

Figure 7.2: Zoning 2: the GEH indicator of link flows produced by method 2.2 on the Sioux Falls case study 


\section{A.1.2 Method 3: standard assignment with capacity reduction of intrazonal links}

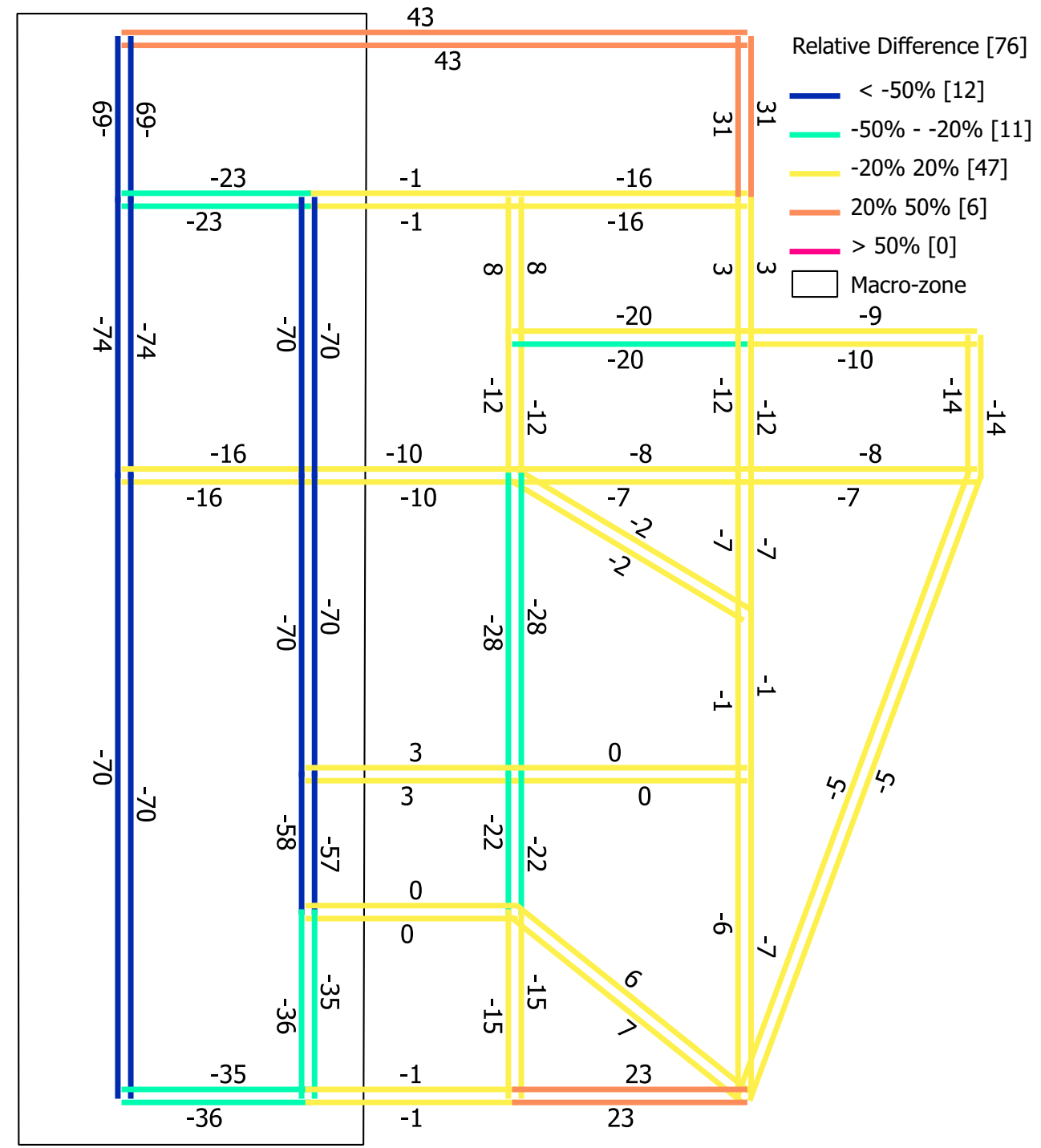

Figure 7.3: Zoning 2: the relative difference (RD) of link flows produced by method 3 on the Sioux Falls case study 


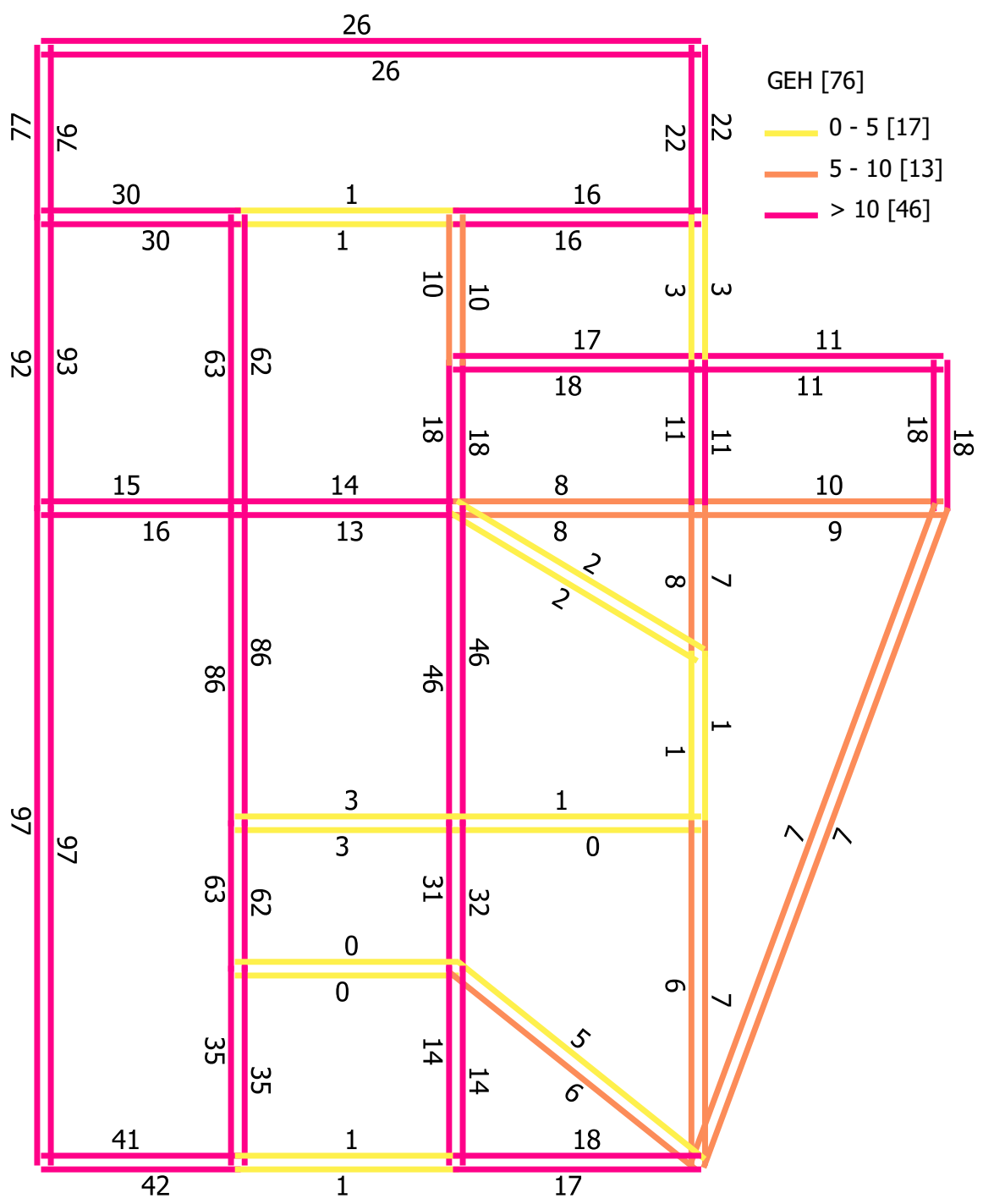

Figure 7.4: Zoning 2: the GEH indicator of link flows produced by method 3 on the Sioux Falls case study 


\section{A.2 Zoning 1: zones 1, 3, 4, 11, 12}

This zoning produces $2.05 \%$ of intrazonal trips.

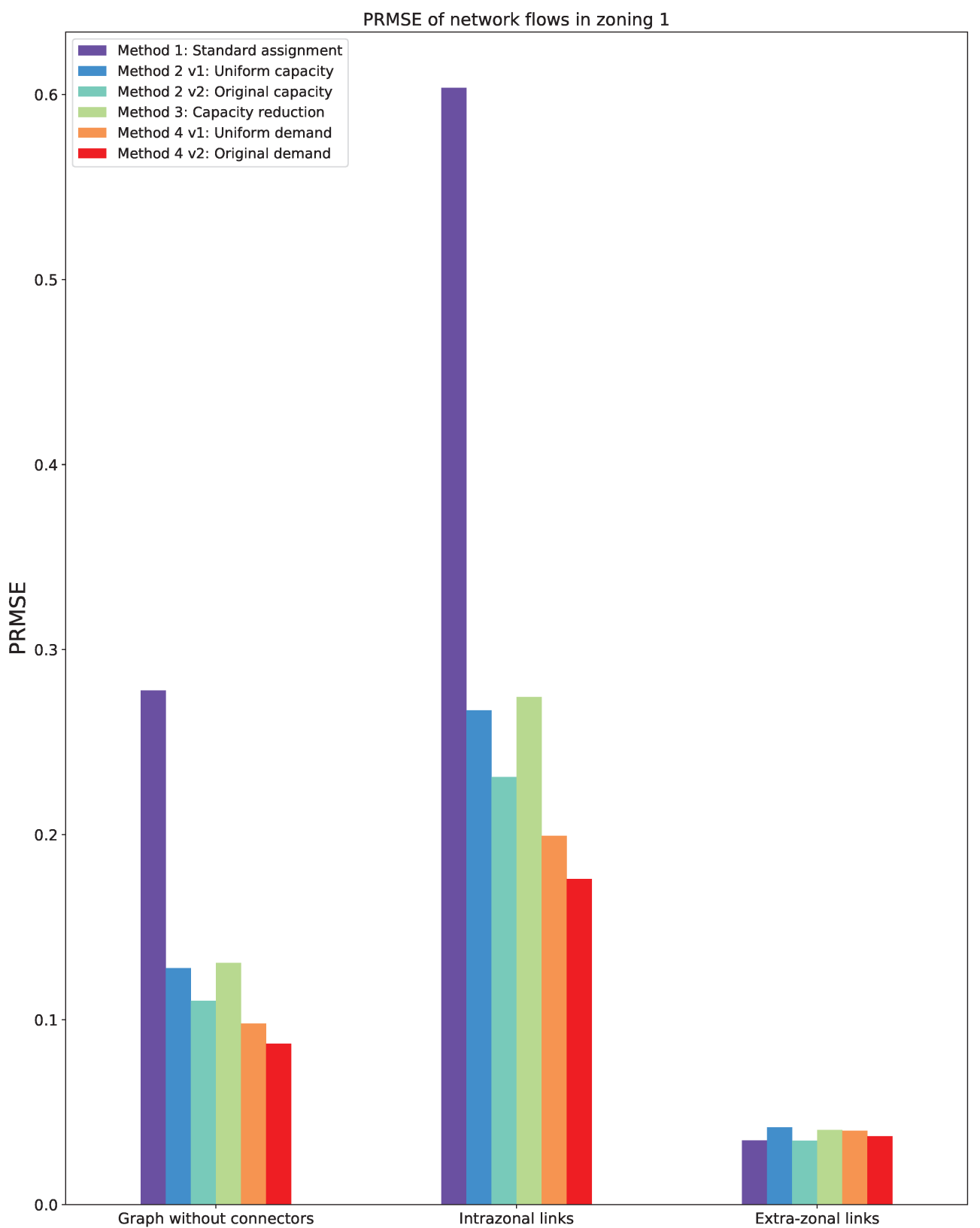

Figure 7.5: Zoning 1: PRMSE of assignment results on the Sioux Falls case study 


\section{A.3 Zoning 3: zones 1, 2, 3, 4, 5, 6, 7, 8, 9, 10}

This zoning produces $14.14 \%$ of intrazonal trips.

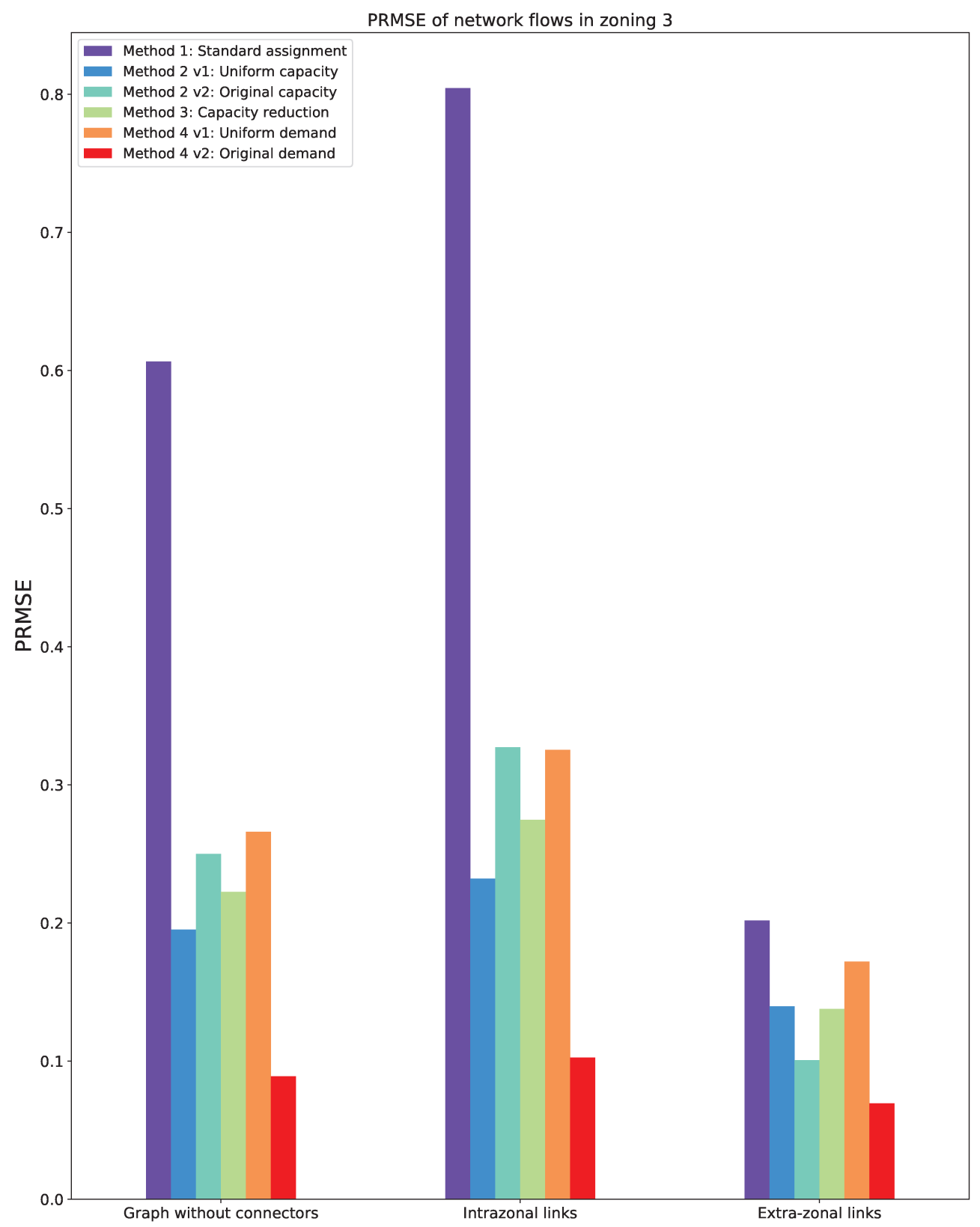

Figure 7.6: Zoning 3: PRMSE of assignment results on the Sioux Falls case study 


\section{A.4 Zoning 4: zones 13, 14, 15, 19, 20, 21, 22, 23, 24}

This zoning produces $17.94 \%$ of intrazonal trips.

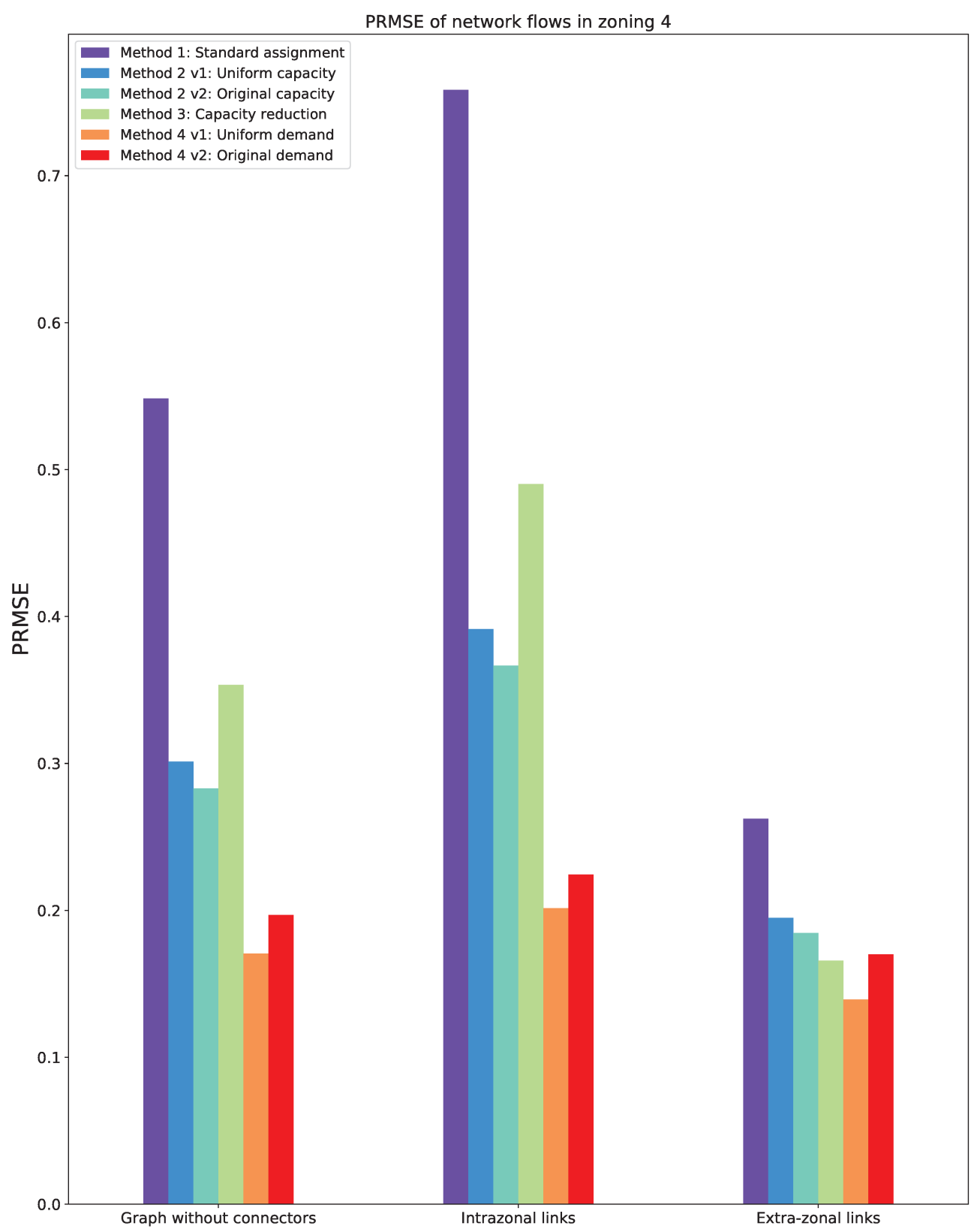

Figure 7.7: Zoning 4: PRMSE of assignment results on the Sioux Falls case study 


\section{A.5 Zoning 5: zones 2, 5, 6, 7, 8, 9, 10, 16, 17, 18}

This zoning produces $22.41 \%$ of intrazonal trips.

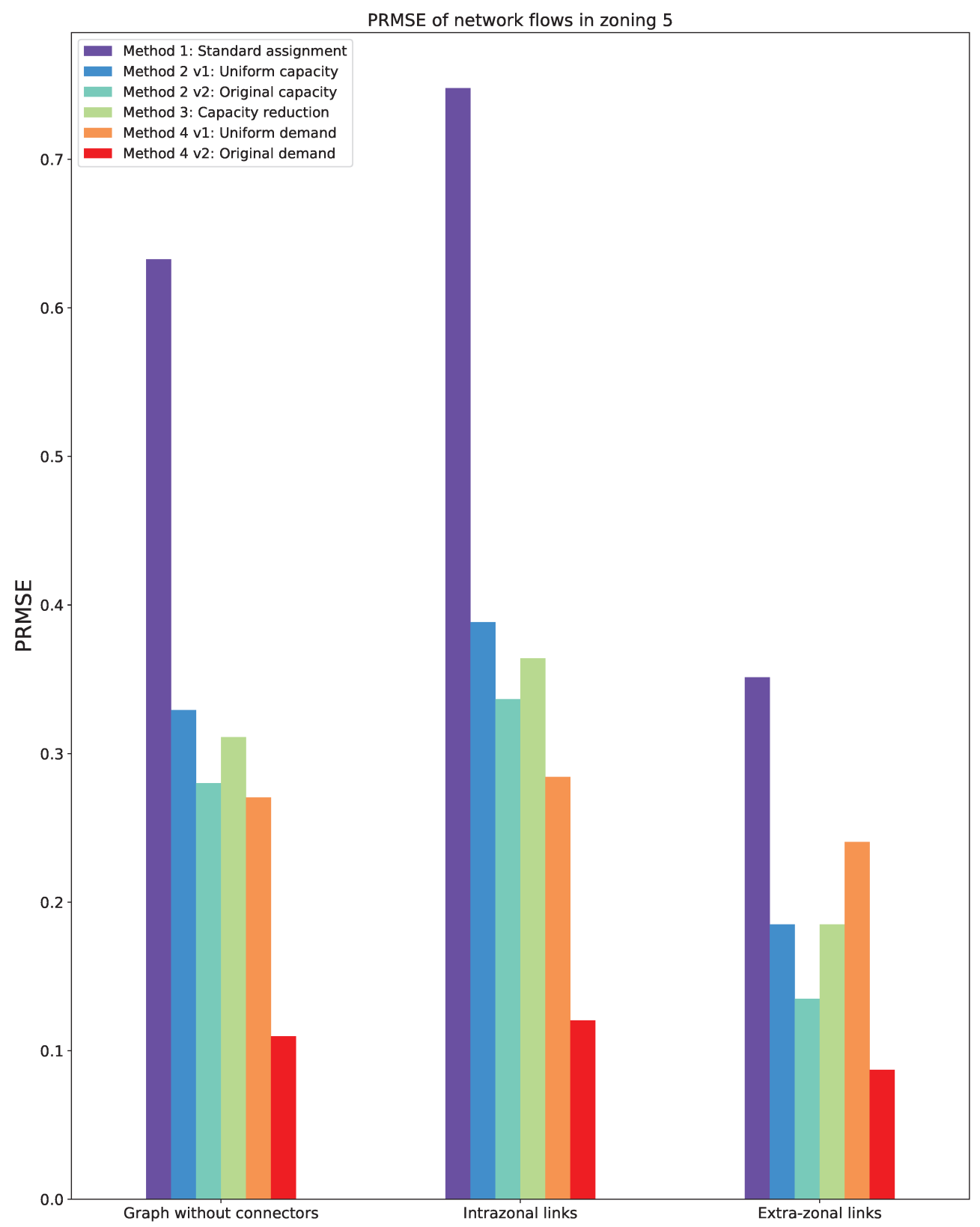

Figure 7.8: Zoning 5: PRMSE of assignment results on the Sioux Falls case study 


\section{B GEH indicator for the case study of Lyon}

The GEH indicator is a goodness-of-fit measure computed for each network link and each simulation. In order to get a global indicator from each traffic assignment, the distribution of the GEH is summarized by its median GEH. The distribution of the mean is similar to that of the median. Since the purpose of our research is more about the study of the relative contribution of new assignment strategies than the calibration/validation of operational models, GEH recommendations are considered for informative purposes only.

The distribution of median GEH is similar to that of the PRMSE: methods 4.1 and 4.2 are found to improve the quality of assignment outcomes and to reproduce more accurately reference flows than the standard assignment. For highway, arterial, and major collector roads, method 4.1 has the lowest median GEH when the rate of intrazonal trips is greater than 20\%. Method 4.2 is more accurate on low road hierarchies and especially on category 5 and with detailed zonings.

On highway roads, method 4.1 produces more accurate results than method 4.2 when the rate of intrazonal demand is greater than $20 \%$. Out of 700 simulations, method 4.1 outperforms method 4.2 on 400 cases. Method 4.2 is however more accurate than 4.1 in simulations where the rate of intrazonal trips is low.

The outperformance of method 4.1 becomes less obvious as one goes further in road hierarchy. On arterial and major collector roads, this strategy still produce the most accurate results but only on 200 simulations over 700 . The rest of simulations are more accurately modeled by method 4.2 .

On road hierarchy 5, method 4.2 outweighs method 4.1 in all simulations. Flows of local streets are reproduced accurately by the original method and the maximum GEH is less than 6 for all simulations.

The above findings suggest that the new assignment strategies can have a significant contribution to the calibration and validation of transport models by significantly reducing modeling errors. 


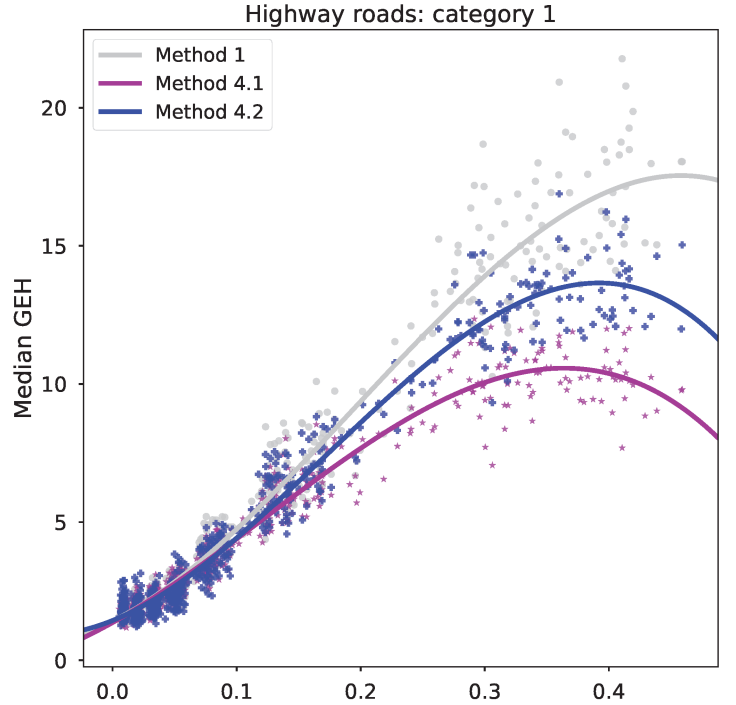

(a) Intrazonal trips rate

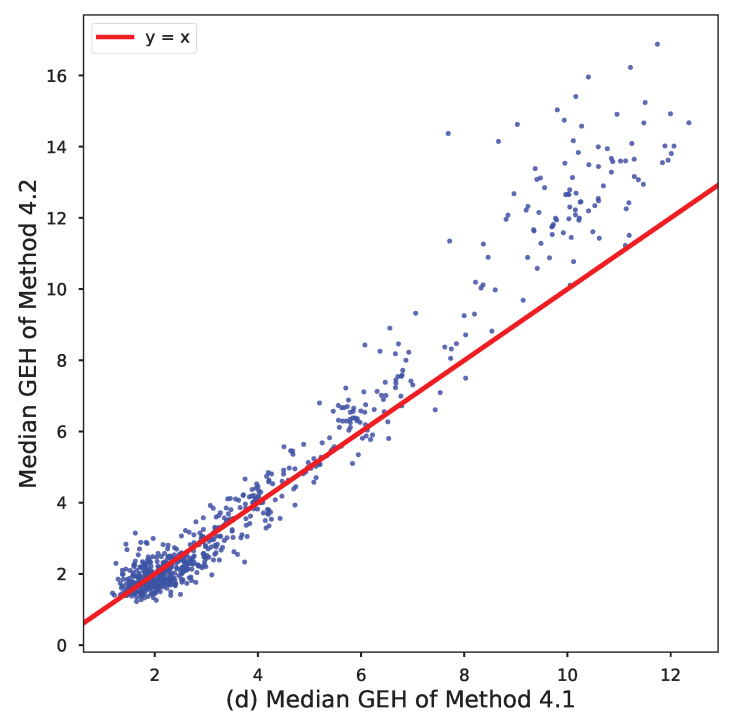

Arterial roads: category 2

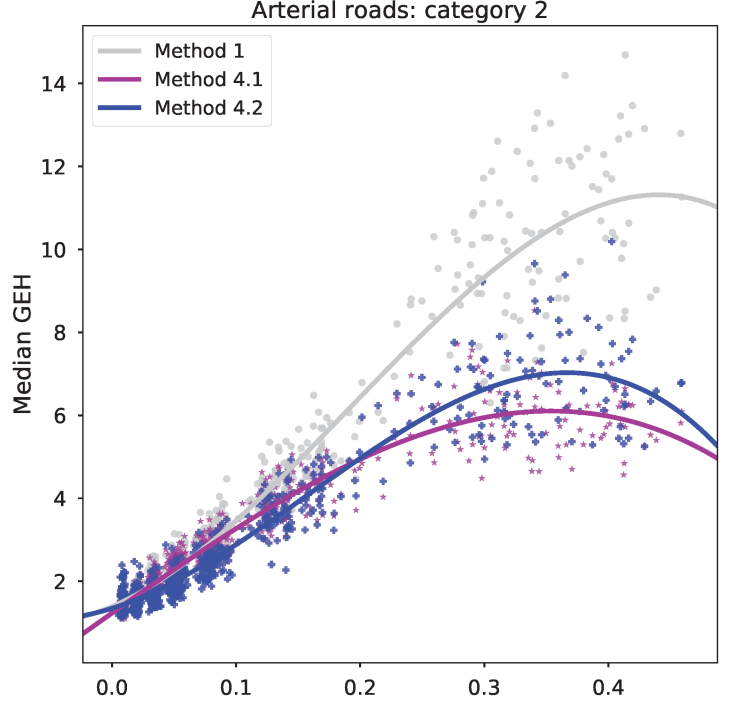

(b) Intrazonal trips rate

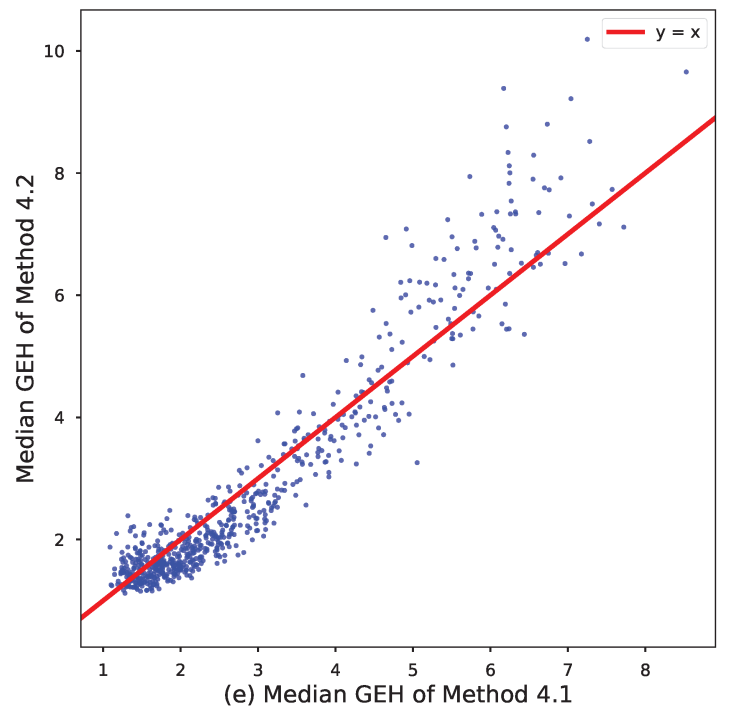

Major collector roads: category 3

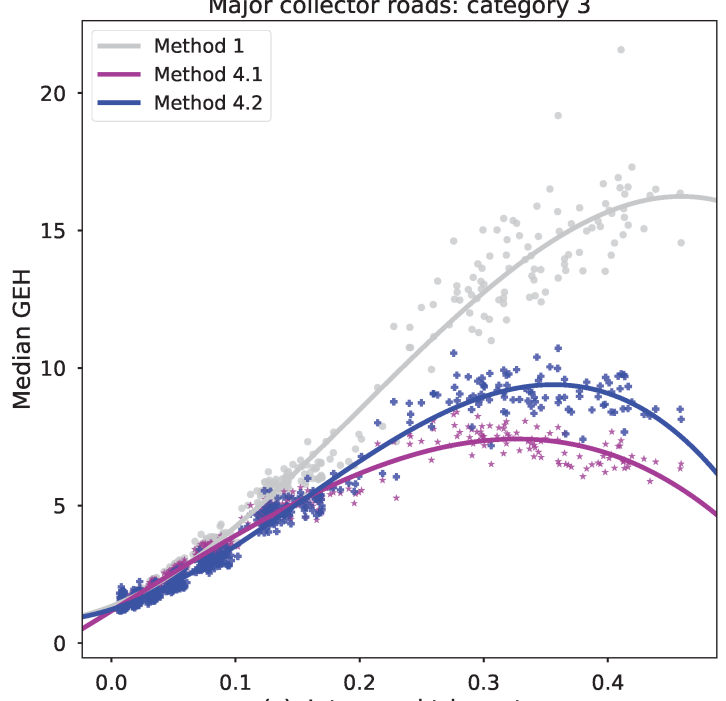

(c) Intrazonal trips rate

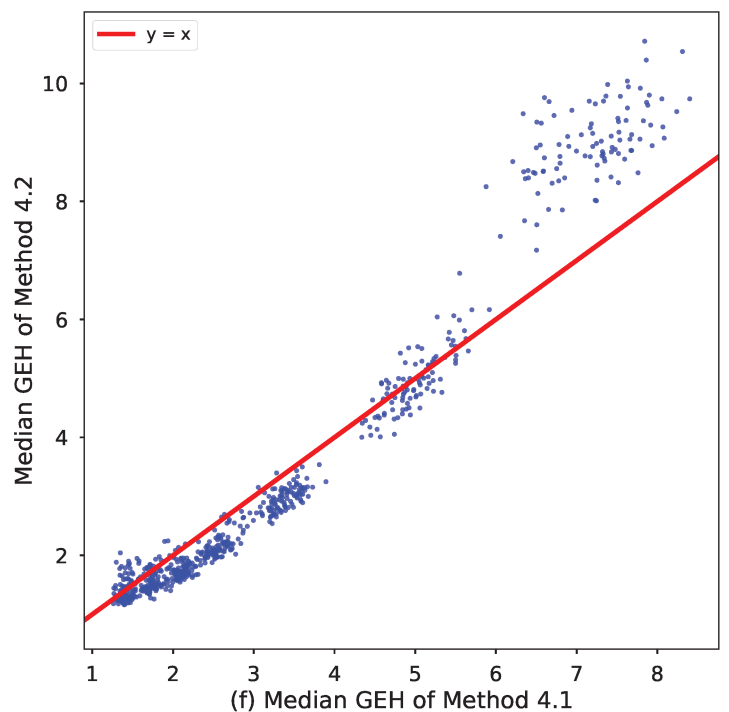

Figure 7.9: Median GEH indicator of the three assignment methods on road categories 1, 2, and 3 

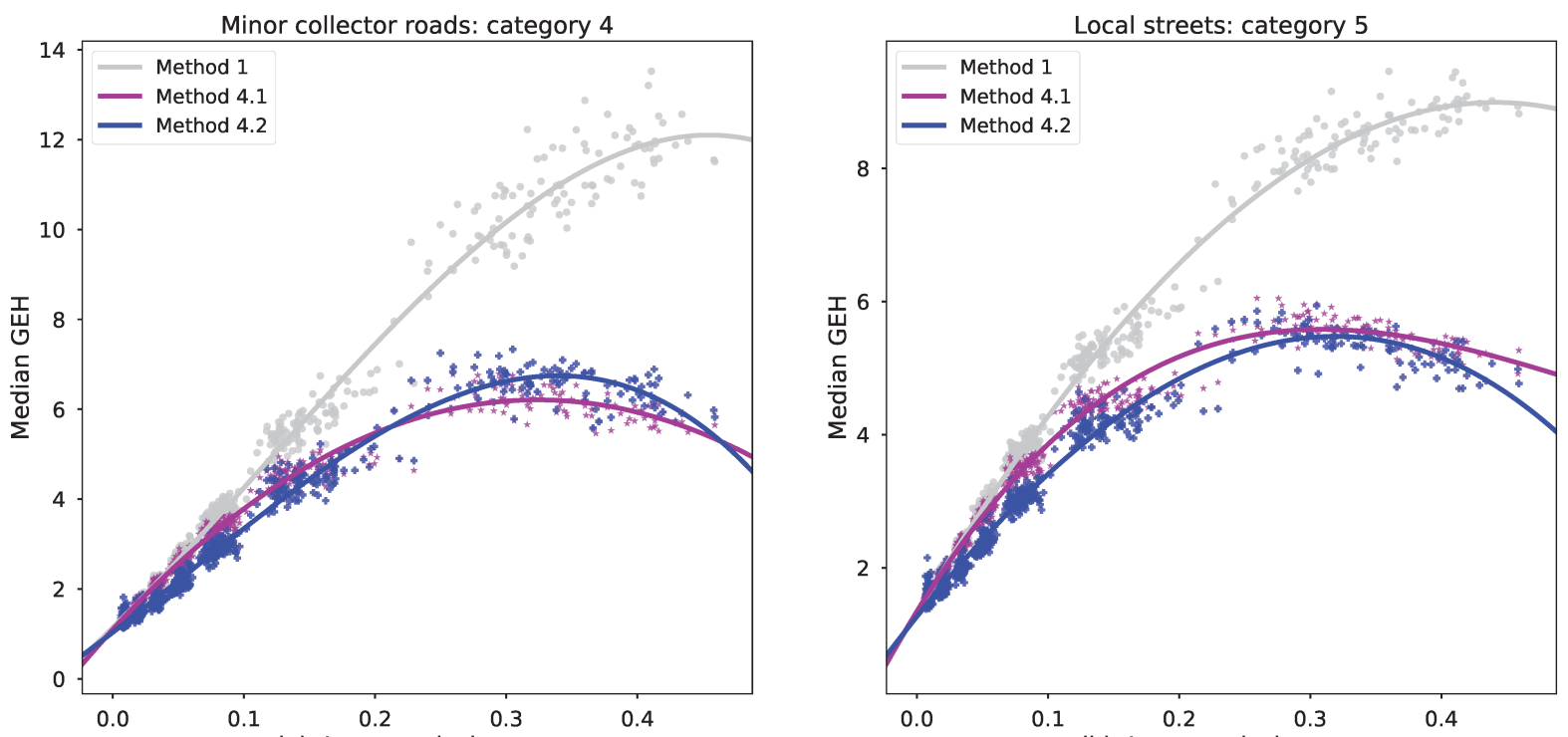

(a) Intrazonal trips rate
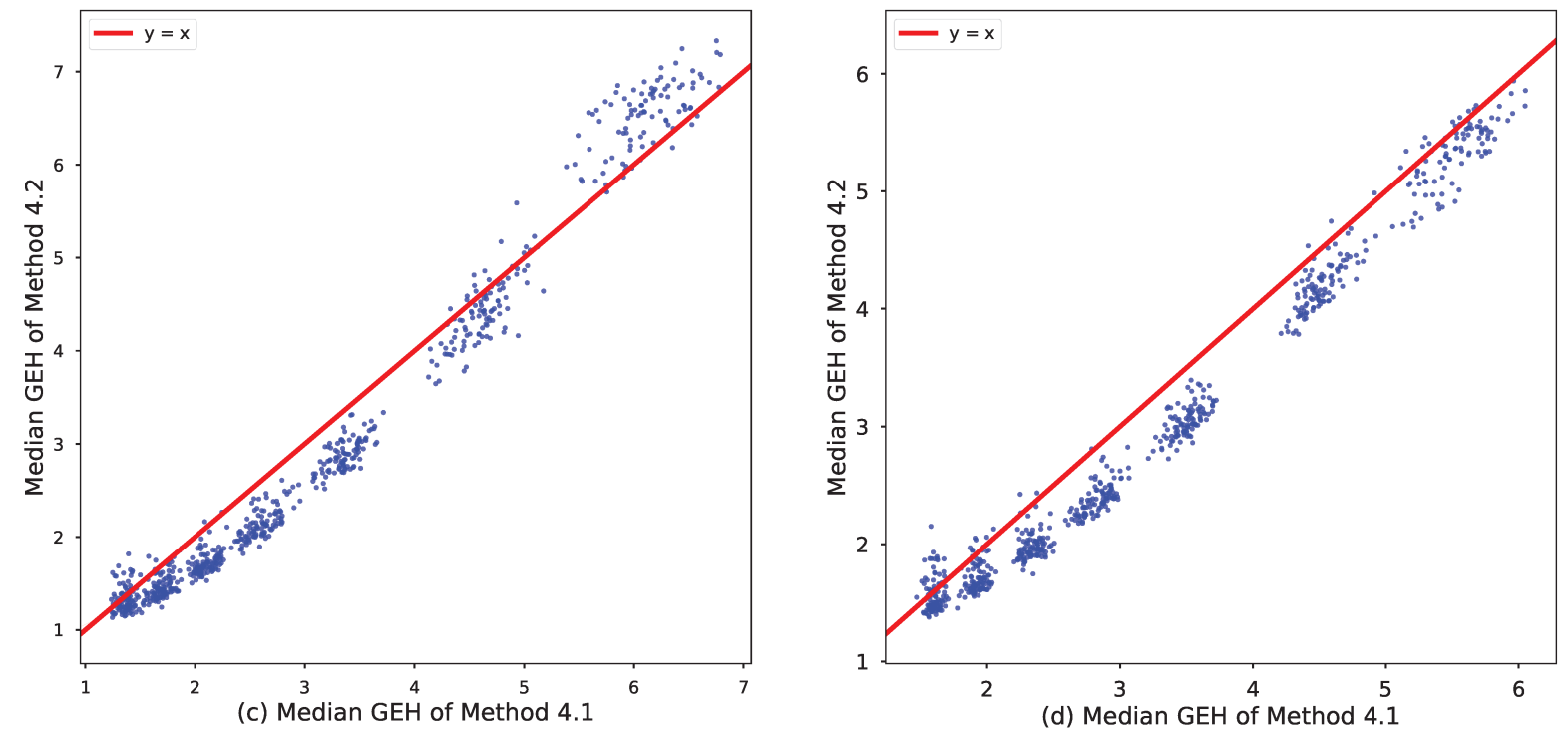


\section{Content}

1 Introduction $\quad 17$

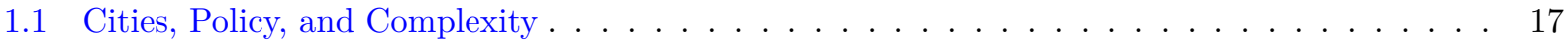

1.2 Urban models as a support-aid tool . . . . . . . . . . . . . . . . . . . . . . . . . . . . 19

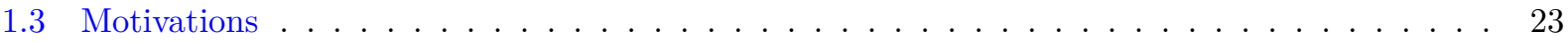

1.4 Research framework: the aggregation problem in LUTM . . . . . . . . . . . . . . 25

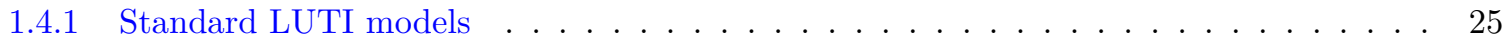

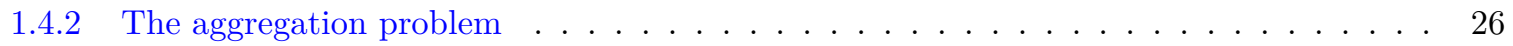

1.4.3 The spatial aggregation problem $\ldots \ldots \ldots \ldots \ldots \ldots \ldots \ldots \ldots$

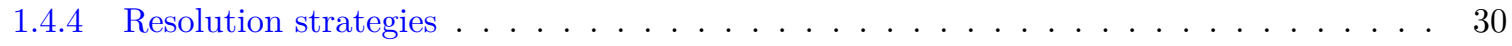

1.4.5 Four Step Models and the spatial aggregation bias . . . . . . . . . . . . . . . 32

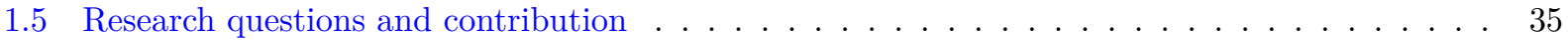

1.5.1 Research questions . . . . . . . . . . . . . . . . . . . . . 35

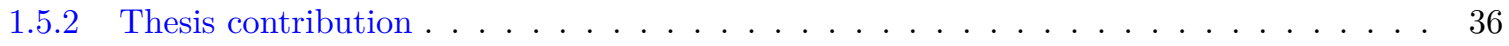

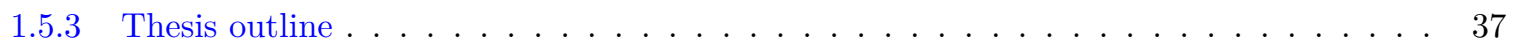

2 Sensitivity analysis on connectors $\quad 41$

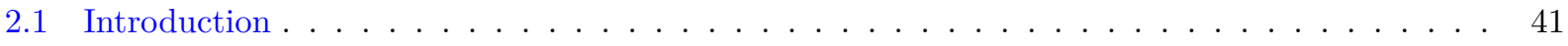

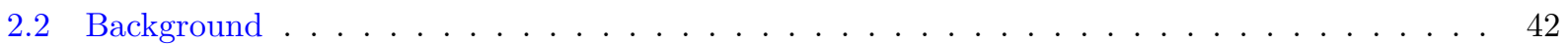

2.3 Methods . . . . . . . . . . . . . . . . . . . . . . . 45

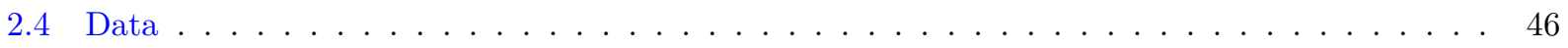

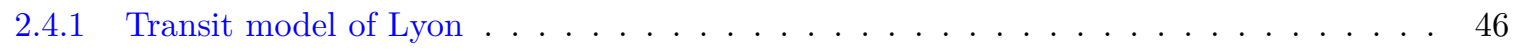

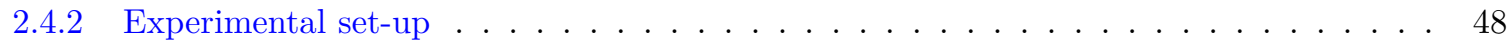

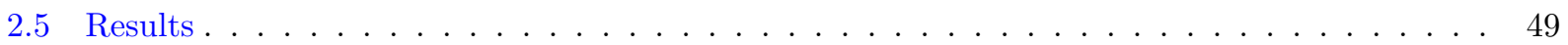


2.5.1 Impact of connectors on the overall transit ridership . . . . . . . . . . . . . . 49

2.5.2 The impact of connectors on modal ridership . . . . . . . . . . . . . . . 51

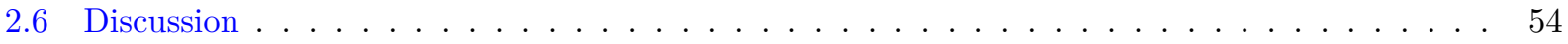

2.6.1 Correlation between transit ridership and $N_{\max } \ldots \ldots \ldots \ldots \ldots \ldots$

2.6.2 Correlation between transit ridership and $L_{\max } \ldots \ldots \ldots \ldots \ldots \ldots \ldots$

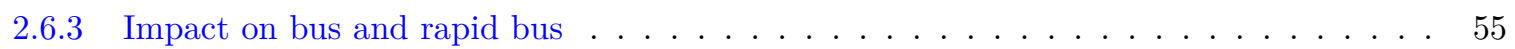

2.6 .4 Decreasing variability of results $\ldots \ldots \ldots \ldots \ldots \ldots \ldots \ldots$

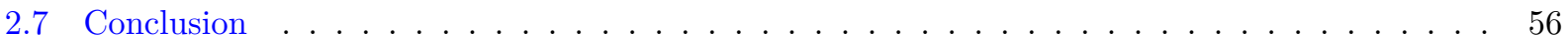

$\begin{array}{lll}3 & \text { A new definition of transit connectors } & 57\end{array}$

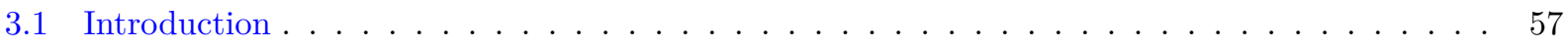

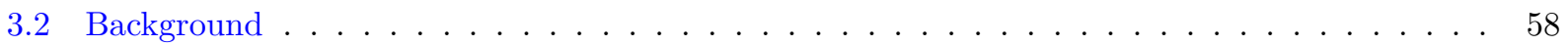

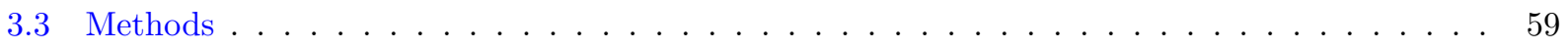

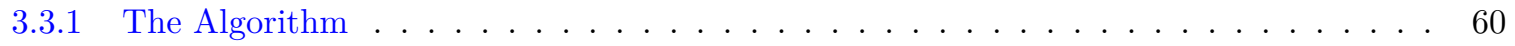

3.3 .2 Justification . . . . . . . . . . . . . . . . . . . . . 62

3.4 A case study of Lyon . . . . . . . . . . . . . . . . . . . . . 66

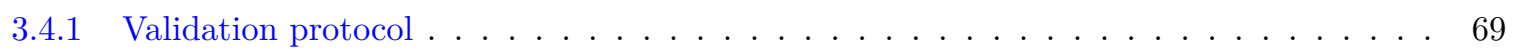

3.4 .2 Results and discussion $\ldots \ldots \ldots \ldots \ldots \ldots \ldots \ldots$

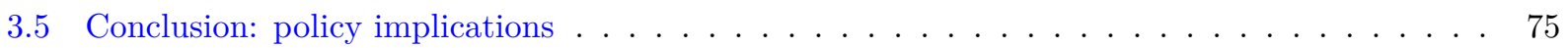

4 The omission of intrazonal trips $\quad 79$

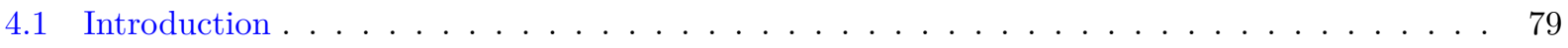

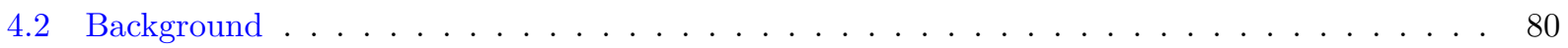

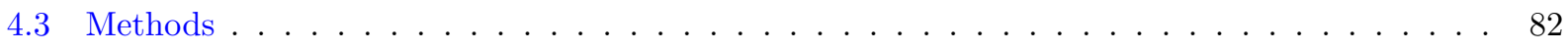

4.3 .1 Zonal Aggregation . . . . . . . . . . . . . . . . . . . . 84

4.3 .2 Traffic Assignment . . . . . . . . . . . . . . . . . 86

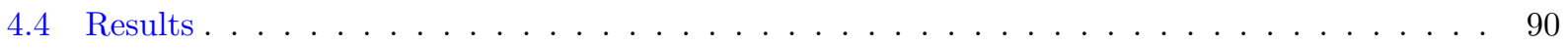

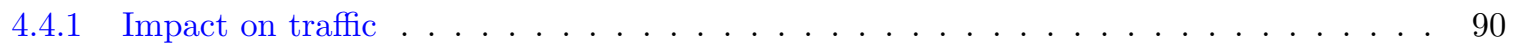

4.4 .2 Impact on journey speeds $\ldots \ldots \ldots \ldots \ldots \ldots \ldots \ldots \ldots \ldots$

4.4.3 Impact on congestion ratio and total vehicle-kilometers of travel $\ldots \ldots \ldots \ldots$

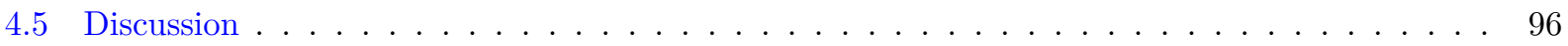




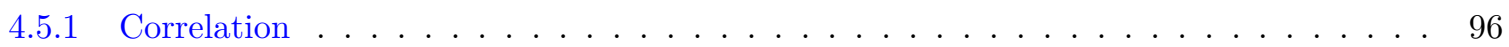

4.5.2 Asymptotic effect or threshold effect . . . . . . . . . . . . . . . . . . . . . 99

4.5.3 Impact on the secondary network . . . . . . . . . . . . . . . . . . . . . . . 101

4.5.4 Size and shape effects . . . . . . . . . . . . . . . . . . . . 101

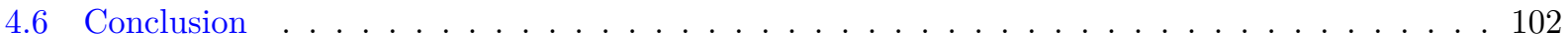

5 Spatial aggregation in assignment 105

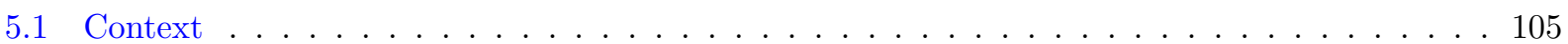

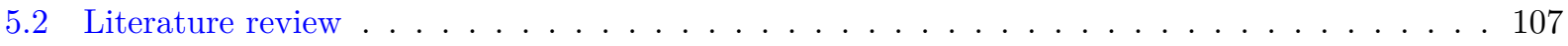

5.2 .1 Demand-side methods . . . . . . . . . . . . . . . . . . . . . . 107

5.2 .2 Supply-side methods . . . . . . . . . . . . . . . . . . . . . . . . . . 109

5.2 .3 Mix methods . . . . . . . . . . . . . . . . . . . . . . . 110

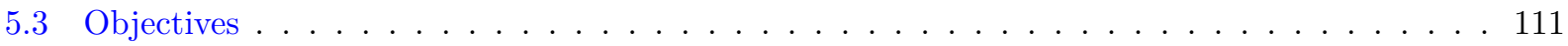

5.4 Methods . . . . . . . . . . . . . . . . . . . . . . . . . 111

5.4 .1 Traffic assignment strategies . . . . . . . . . . . . . . . . . . . . 113

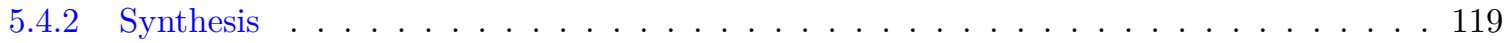

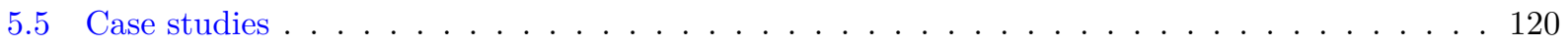

5.5.1 Simple case study: a 4 zones 8 links example . . . . . . . . . . . . . . . . . 120

5.5 .2 Sioux Falls case study $\ldots \ldots \ldots \ldots \ldots \ldots$

5.5 .3 Lyon case study . . . . . . . . . . . . . . . . . . . . . . . . . 122

5.6 Results . . . . . . . . . . . . . . . . . . . . . . . . . 125

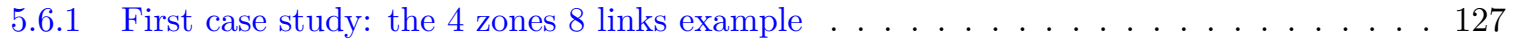

5.6 .2 Sioux Falls case study $\ldots \ldots \ldots \ldots \ldots \ldots \ldots \ldots$

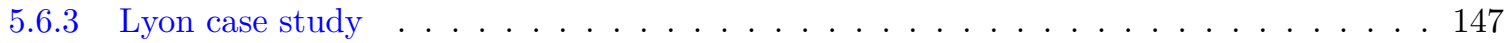

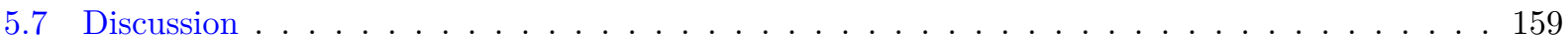

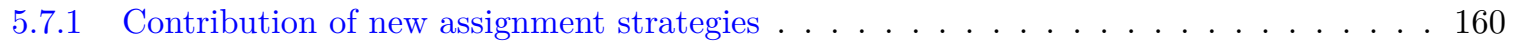

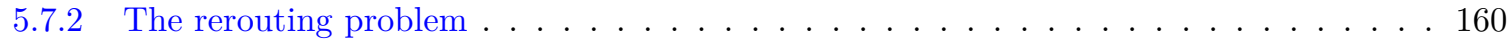

5.7 .3 Why a U-shape? . . . . . . . . . . . . . . . . . . . . 163

5.7.4 The artifact behind the outperformance of method 4.1 over $4.2 \ldots \ldots \ldots$

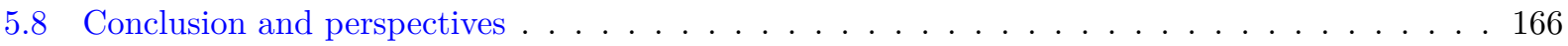


6 General discussion $\quad 171$

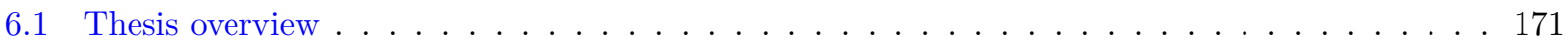

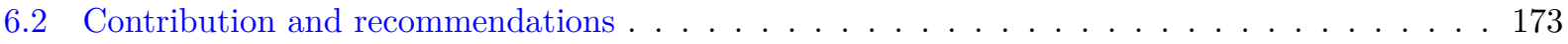

6.2 .1 A new definition of transit connectors . . . . . . . . . . . . . . . . 173

6.2.2 The impact of ignoring intrazonal trips in traffic assignment models . . . . . . . . . 175

6.2.3 The spatial aggregation problem in traffic assignment models . . . . . . . . . . . . 178

6.2 .4 General contribution . . . . . . . . . . . . . . . . . . . 180

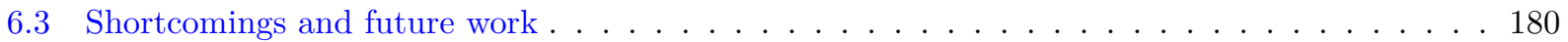

6.3 .1 Incomplete work . . . . . . . . . . . . . . . . . . . . . 180

6.3.2 Spatial aggregation, detailed data, and microsimulation $\ldots \ldots \ldots \ldots \ldots$

6.3 .3 Global contribution . . . . . . . . . . . . . . . . . . . . . . 182

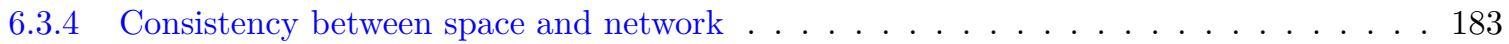

$\begin{array}{lr}\text { References } & 185\end{array}$

$\begin{array}{llr}7 & \text { Appendix } & 201\end{array}$

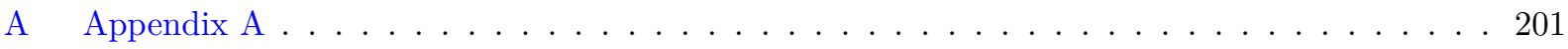

A.1 Zoning 2: zones $1,3,4,11,12,13,14,23,24 \ldots \ldots \ldots \ldots \ldots \ldots \ldots \ldots \ldots \ldots$

A.2 Zoning 1: zones $1,3,4,11,12 \ldots \ldots \ldots \ldots \ldots$

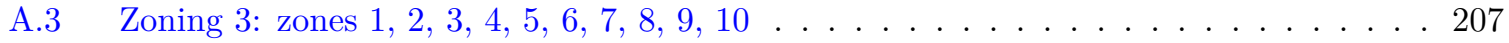

A.4 Zoning 4: zones $13,14,15,19,20,21,22,23,24 \ldots \ldots \ldots$

A.5 Zoning 5: zones $2,5,6,7,8,9,10,16,17,18 \ldots \ldots \ldots \ldots$

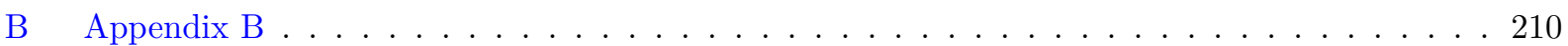




\section{List of figures}

1.1 Conventional Four Step Model architecture . . . . . . . . . . . . . . . . . . . 20

1.2 Land-Use and Transport interaction models according to Wegener (1998) . . . . . . . . . 21

1.3 Research summary . . . . . . . . . . . . . . . . . . . . . . . . . 39

2.1 The standard definition of centroid connectors. Only the $N$ nearest stopping points within a buffer of radius $L$ around zone centroid are connected. . . . . . . . . . . . . . . . 42

2.2 The Metropolitan Area of Lyon and its transit system . . . . . . . . . . . . . 47

2.3 Impact of transit connectors on total transit ridership. $\mathrm{X}$-axis depicts the maximum number of connectors $N_{\max }$. The Y-axis depicts corresponding transit ridership. Three values are used for $L_{\max }: 500,1,000$, and 1,500 meters $\ldots \ldots \ldots \ldots \ldots \ldots$

2.4 The p-value of statistical tests of $H_{0}$ and $H_{0}^{\prime}$ on mean and variance equality. X-axis depicts the maximum number of connectors $N_{\max } \ldots \ldots \ldots \ldots \ldots$

2.5 Statistical distribution of the impact of transit connectors on total ridership of (a) bus and (b) rapid bus systems. $\mathrm{X}$-axis depicts the maximum number of connectors $N_{\max }$. The $\mathrm{Y}$-axis depicts corresponding transit ridership. Three values are used for $L_{\max }: 500,1,000$, and 1,500

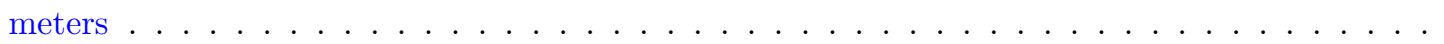

2.6 The statistical distribution of the impact of transit connectors on total ridership of (a) light rail and (b) subway systems. X-axis depicts the maximum number of connectors $N_{\max }$. The Y-axis depicts corresponding transit ridership. Three values are used for $L_{\max }: 500,1,000$,

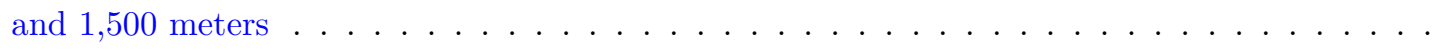

2.7 Impact of transit connectors on transfers of all transit modes. $\mathrm{X}$-axis depicts the maximum number of connectors $N_{\max }$. The Y-axis depicts corresponding transit transfers. Three values are used for $L_{\max }: 500,1,000$, and 1,500 meters $\ldots \ldots \ldots \ldots \ldots \ldots$

3.1 The new definition of transit connectors: the algorithm $\ldots \ldots \ldots \ldots$

3.2 Selection of connection nodes. Transit routes A and B serve 4 transit stops and 4 zones. Route A serves stops S1-A, S2-A and S3-A. Route B serves stops S3-B and S4-B . . . . . . . . .

3.3 The Urban Area of Lyon and its transit system (limits of 1999) (Sources IGN, Métropole de

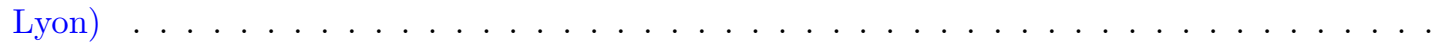

3.4 Distance decay functions by transit mode (Source: RATP data) . . . . . . . . . . . . 68

3.5 Observed and estimated total transit journeys by mode. The bars are displayed in the order of

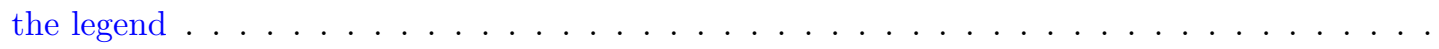

3.6 PRMSE between predicted and observed transit ridership by mode. The bars are displayed in

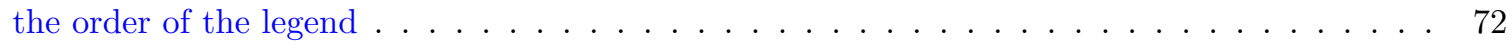

3.7 The ratio between observed and estimated passenger volumes (ROEPV) $\ldots \ldots \ldots \ldots$ 
4.1 The experimental protocol. The study area is divided using different spatial designs. For each zoning an assignment procedure is computed and the variability of results is analyzed . . . .

4.2 The urban area of Lyon (limits of 1999). The urban area is divided into 777 TAZ (IRIS) (data produced by Laboratoire Aménagement Économie Transports and IGN: Institut national de l'information géographique et forestière $) \ldots \ldots \ldots \ldots \ldots$. . . . . . . . . 86

4.3 The transport network of the Urban Area of Lyon $\ldots \ldots \ldots \ldots$

4.4 Conservation of connectors during zonal aggregation. Four zones: A, B, C, and D connected to their corresponding connection nodes: a, b, c, and d with connectors A-a, B-b, C-c, and $\mathrm{D}-\mathrm{d}$ respectively. The connectors of macro-zone $\mathrm{E}$ are the set of the connectors of micro-zones $\mathrm{C}$ and D (Source: adapted from a suggestion of a reviewer of the paper) . . . . . . . . .

4.5 Correlation between average link flows and intrazonal trips according to road category. $\mathrm{X}$-axis represents the rate of un-assigned intrazonal trips and $\mathrm{Y}$-axis depicts average link flows on the network. The mean is computed using the length of links as a weight. Each point in the graphic is a traffic assignment outcome . . . . . . . . . . . . . . . . . .

4.6 Impact of the level of zonal aggregation on average links flows according to road category. $\mathrm{X}$-axis represents zonal aggregation level and $\mathrm{Y}$-axis depicts average link flows. The mean is computed using the length of links as a weight. For each level of aggregation, 50 alternatives

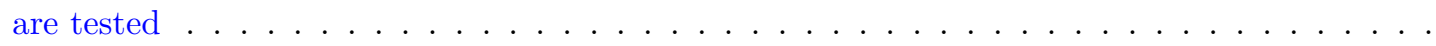

4.7 Correlation between average link speeds and intrazonal trips according to road category. X-axis represents the rate of the un-assigned intrazonal trips and Y-axis depicts average link speed. The mean is computed using the length of links as a weight. Each point in the graphic is a traffic assignment outcome . . . . . . . . . . . . . . . . .

4.8 Impact of level of zonal aggregation on average link speeds according to road category. $\mathrm{X}$-axis represents zonal aggregation level and Y-axis depicts average link speed. The mean is computed using the length of links as a weight. For each level of aggregation, 50 alternatives are tested

4.9 Correlation between congestion ratio and intrazonal trips according to road category. X axis represents the rate of the un-assigned intrazonal trips and $\mathrm{Y}$ axis depicts congestion ratio. The mean is computed using the length of links as a weight. Each point in the graphic is a traffic

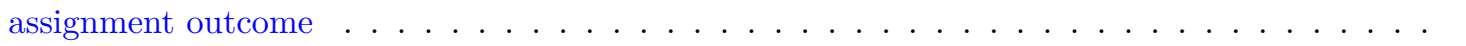

4.10 Correlation between total vehicle kilometers of travel (VKT) and intrazonal trips according to road category. $\mathrm{X}$ axis represents the rate of the un-assigned intrazonal trips and $\mathrm{Y}$ axis depicts total VKT. Each point in the graphic is a traffic assignment outcome . . . . . . . . 98

4.11 Intrazonal trips and zonal aggregation levels. For each level of aggregation, 50 alternative spatial designs are constructed . . . . . . . . . . . . . . . . . . 100

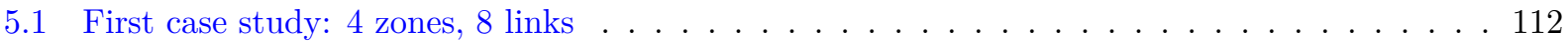

5.2 A model of traffic assignment models . . . . . . . . . . . . . . . . . . . 113

5.3 Sioux Falls network Wang et al. (2013) . . . . . . . . . . . . . . . . . . . . . . 121

5.4 Original zoning of the case study of Lyon $\ldots \ldots \ldots \ldots \ldots$ 


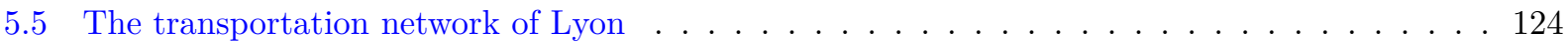

5.6 Distribution of the rate of intrazonal trips according to the aggregation level $\ldots \ldots \ldots$

5.7 Simple case: PRMSE of link flows of the 6 assignment strategies. Color bars are displayed in the order of the legend. The PRMSE of Method 4.2 is nearly $0 \ldots \ldots \ldots \ldots$

5.8 Sioux Falls: original link flows. Link flows may differ from those known in the literature due to differences in convergence criteria $\ldots \ldots \ldots \ldots \ldots \ldots \ldots$

5.9 Sioux Falls: the relative difference (RD) of link flows produced by method 1 . . . . . . . 139

5.10 Sioux Falls: the relative difference $(\mathrm{RD})$ of link flows produced by method $2.1 \ldots \ldots 141$

5.11 Sioux Falls: the relative difference $(\mathrm{RD})$ of link flows produced by method $4.1 \ldots$. . . . 143

5.12 Sioux Falls: the relative difference (RD) of link flows produced by method $4.2 \ldots \ldots 144$

5.13 Sioux Falls: the PRMSE of link flows of the six assignment methods. Color bars are displayed

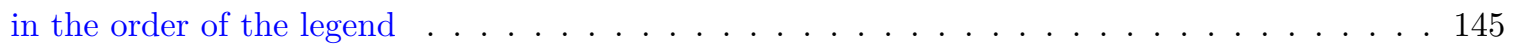

5.14 Lyon: correlation between link flows and intrazonal trips of method 1 according to road hierarchy. The $\mathrm{X}$-axis represents the rate of intrazonal trips and $\mathrm{Y}$-axis depicts average link flows. The mean is computed using the length of links as a weight $\ldots \ldots \ldots \ldots$

5.15 Lyon: correlation between Vehicle-Kilometers of Travel (VKT) and intrazonal trips on the overall network of assignment method 1 . The $\mathrm{X}$-axis represents the rate of intrazonal trips and Y-axis depicts total vehicle-kilometers of travel on the network $\ldots \ldots \ldots \ldots \ldots$

5.16 Lyon: correlation between link flows and intrazonal trips of methods 1 and 4.1 according to road hierarchy. The $\mathrm{X}$-axis represents the rate of intrazonal trips and $\mathrm{Y}$-axis depicts average link flows. The mean is computed using the length of links as a weight . . . . . . . . . 152

5.17 Lyon: Correlation between link flows and intrazonal trips of methods 1, 4.1, and 4.2 according to road hierarchy. The $\mathrm{X}$-axis represents the rate of intrazonal trips and $\mathrm{Y}$-axis depicts average link flows. The mean is computed using the length of links as a weight . . . . . . . . . . 154

5.18 Lyon: correlation between Vehicle-Kilometers of Travel (VKT) and intrazonal trips of methods 1, 4.1, and 4.2. The $\mathrm{X}$-axis represents the rate of intrazonal trips and $\mathrm{Y}$-axis depicts vehiclekilometers of travel on the network . . . . . . . . . . . . . . . 155

5.19 Lyon: PRMSE of link flows of methods 1, 4.1, and 4.2 on road categories 1, 2, and 3. Each point is a PRMSE value of an assignment simulation . . . . . . . . . . . 157

5.20 Lyon: PRMSE of link flows of methods 1, 4.1, and 4.2 on road categories 4 and 5. Each point is a PRMSE value of an assignment simulation $\ldots \ldots \ldots \ldots \ldots$

5.21 Lyon: significance levels of rejection of the Null Hypothesis of equal means of Welch's t-test . 159

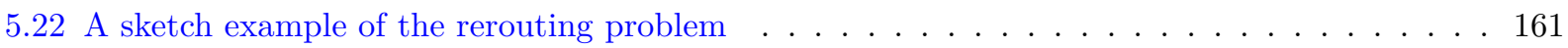

5.23 Lyon: Average Traveled Distance in Km function of intrazonal trips. The X-axis represents the rate of intrazonal trips and Y-axis depicts the average distance traveled by cars in kilometers 162 
5.24 lyon :Total Vehicle-Kilometers of Travel on the network f . . . . . . . . . . . 163

5.25 The U-shape of assignment outcomes . . . . . . . . . . . . . . . . . . . 164

7.1 Zoning 2: the relative difference (RD) of link flows produced by method 2.2 on the Sioux Falls

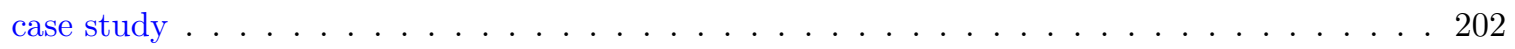

7.2 Zoning 2: the GEH indicator of link flows produced by method 2.2 on the Sioux Falls case study203

7.3 Zoning 2: the relative difference (RD) of link flows produced by method 3 on the Sioux Falls

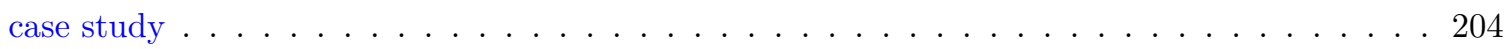

7.4 Zoning 2: the GEH indicator of link flows produced by method 3 on the Sioux Falls case study 205

7.5 Zoning 1: PRMSE of assignment results on the Sioux Falls case study . . . . . . . . . . 206

7.6 Zoning 3: PRMSE of assignment results on the Sioux Falls case study . . . . . . . . . . 207

7.7 Zoning 4: PRMSE of assignment results on the Sioux Falls case study . . . . . . . . . . 208

7.8 Zoning 5: PRMSE of assignment results on the Sioux Falls case study . . . . . . . . . . 209

7.9 Median GEH indicator of the three assignment methods on road categories 1,2 , and $3 \ldots 211$

7.10 Median GEH indicator of the three assignment methods on road categories 4 and $5 \ldots 212$ 


\section{List of tables}

2.1 Network characteristics of the Lyon transit system (GTFS, Métropole de Lyon version 2017) 47

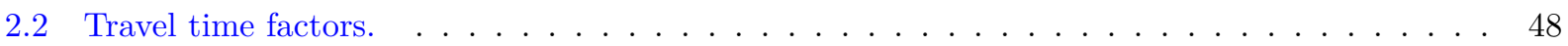

3.1 Accessible connection nodes and routes of the example in figure $3.2 \ldots \ldots \ldots$

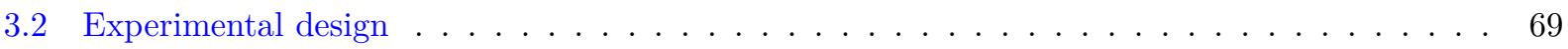

3.3 Transfer rate of the different configurations $\ldots \ldots \ldots \ldots \ldots \ldots \ldots$

4.1 Summary of the current state of knowledge on the impact of intrazonal trips on transport modeling results. Only, relevant papers published before January 2018 are considered. Number

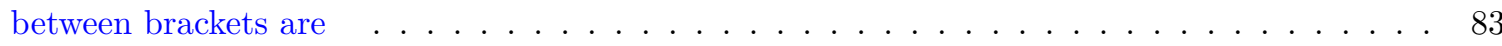

4.2 Characteristics of the network of Lyon according to road hierarchy. Mean values are computed

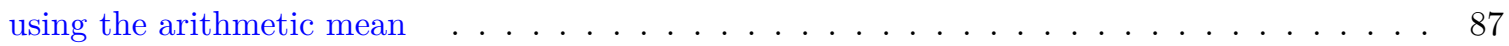

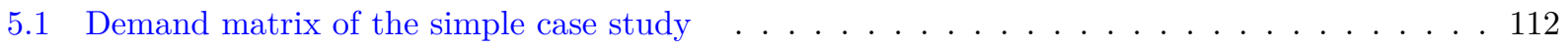

5.2 Uniform capacity of centroid connectors of the simple case study according to method $2.1 \ldots 116$

5.3 Original capacity of centroid connectors of the simple case study according to method $2.2 \ldots 116$

5.4 Trip demand matrix according to method $4.1 \ldots \ldots \ldots \ldots \ldots$

5.5 Trip demand matrix according to method $4.2 \ldots \ldots \ldots \ldots \ldots$

5.6 Synthetic definition of the four assignment methods $\ldots \ldots \ldots \ldots$

5.7 Aggregated spatial designs of the Sioux Falls case study . . . . . . . . . . . . . . . 121

5.8 The hierarchy of the transportation network in the case of Lyon. Average capacity and speed are weighted by the length of links . . . . . . . . . . . . . . . . . 124

5.9 GEH recommendations (Great Britain and Highways Agency, 1996; Smith and Blewitt, 2010) 127

5.10 Simple case study: original assignment results f . . . . . . . . . . . . . . . 127

5.11 Simple case: assignment results of method 1. Reference flows of connector links 5-1 and 5-2 are Not Available $(\mathrm{NA}) \ldots \ldots \ldots \ldots \ldots \ldots \ldots$

5.12 Simple case: assignment results of method 2.1. Reference flows of connector links 5-1 and 5-2 are Not Available $(\mathrm{NA}) \ldots \ldots \ldots \ldots \ldots$. . . . . . . . . . . . . . . . . . . . . . . . . . .

5.13 Simple case: assignment results of method 2.2. Reference flows of connector links 5-1 and 5-2 are Not Available $(\mathrm{NA}) \ldots \ldots \ldots \ldots \ldots \ldots$

5.14 Simple case: assignment results of method 3. Reference flows of connector links 5-1 and 5-2

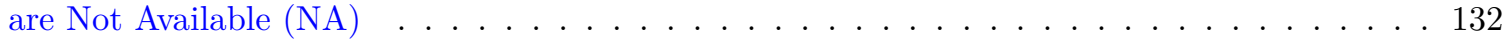

5.15 Simple case: assignment results of method 4.1. Reference flows of connector links 5-1 and 5-2 are Not Available $(\mathrm{NA}) \ldots \ldots \ldots \ldots \ldots \ldots \ldots$ 
5.16 Simple case: assignment results of method 4.2. Reference flows of connector links 5-1 and 5-2 are Not Available $(\mathrm{NA}) \ldots \ldots \ldots \ldots \ldots \ldots$. . . . . . . . . . . . . . . . . . . . . . . . . . .

5.17 Original travel demand of the rerouting example $\ldots \ldots \ldots \ldots \ldots$

5.18 Assignment results of the rerouting example $\ldots \ldots \ldots \ldots \ldots$

5.19 Sioux Falls: descriptive statistics of the distribution of the Absolute Relative Difference (ARD)

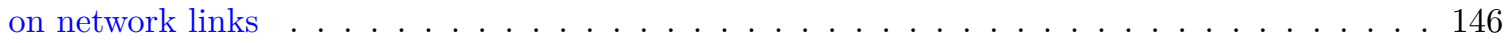

5.20 Sioux Falls: descriptive statistics of the distribution of the GEH on network links $\ldots$. . . 146

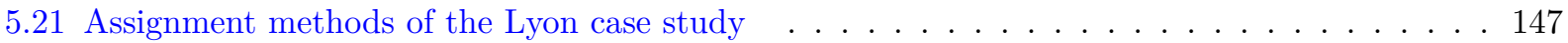

5.22 Descriptive statistics of reference link flows according to road category. Average link flows and deviation are computed using the length of links as a weighting factor $\ldots \ldots \ldots \ldots$

5.23 Lyon case study: additional tests to explore the sensitivity of assignment results towards

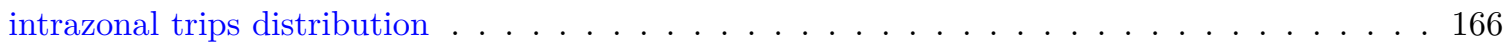

5.24 Assignment results of tests 4.3 and 4.4 compared to original results of methods 4.1 and 4.2 . 166 